WYDZIALEY POLITECHNICZNE KRAKÓW BIBLOTEKA GŁÓWNA

L. inw.

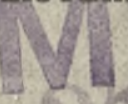

o) 01

\title{
Differential- und \\ Integralrechnung
}

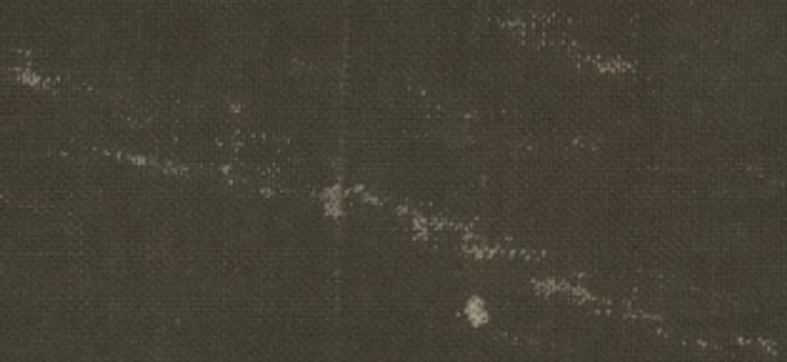


Biblioteka Politechniki Krakowskiej

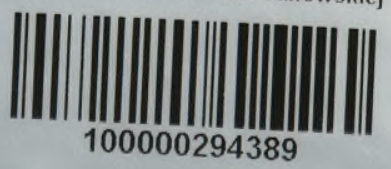




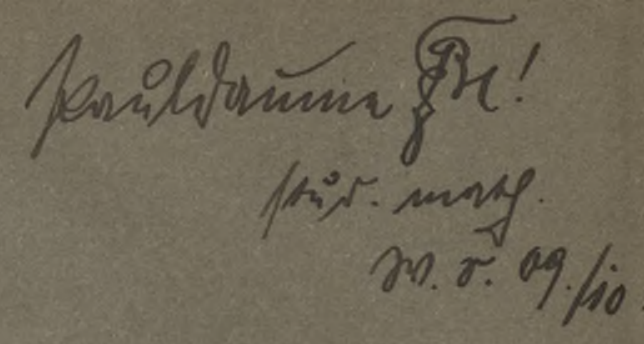


(6)

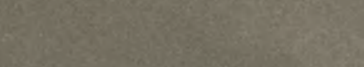

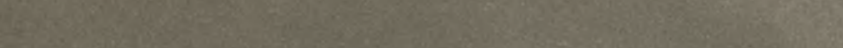

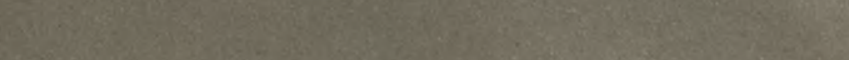
1.7.

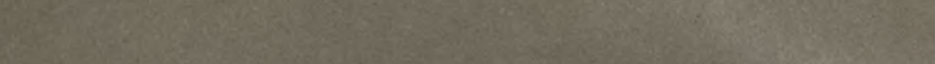

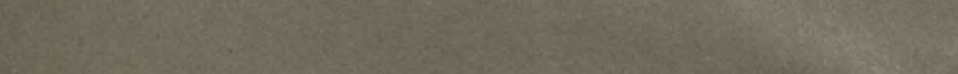

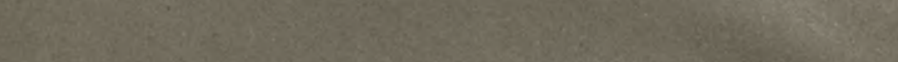
fots 1.

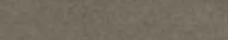
C. E. W.

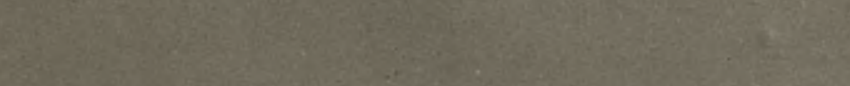

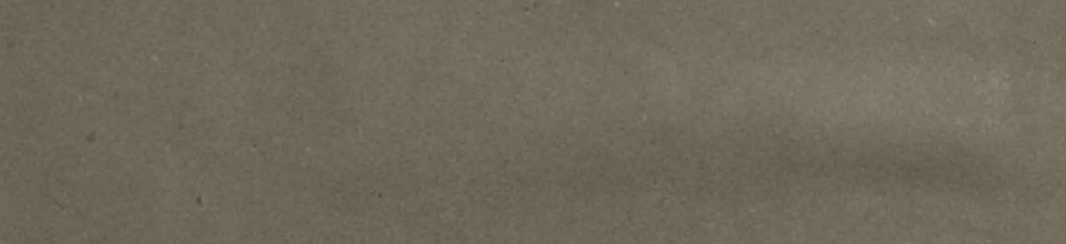

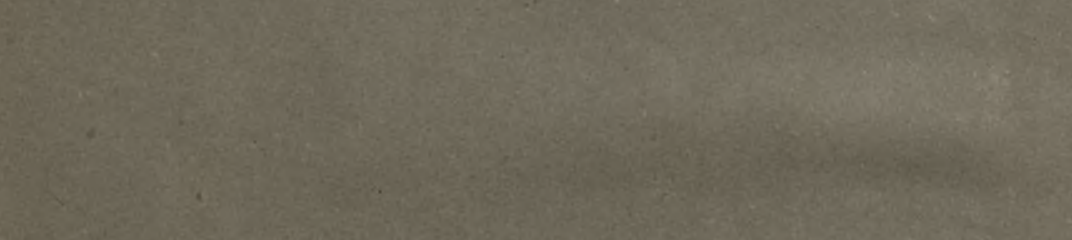

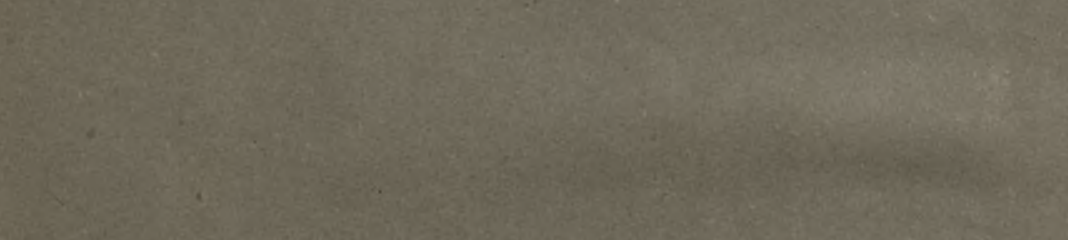

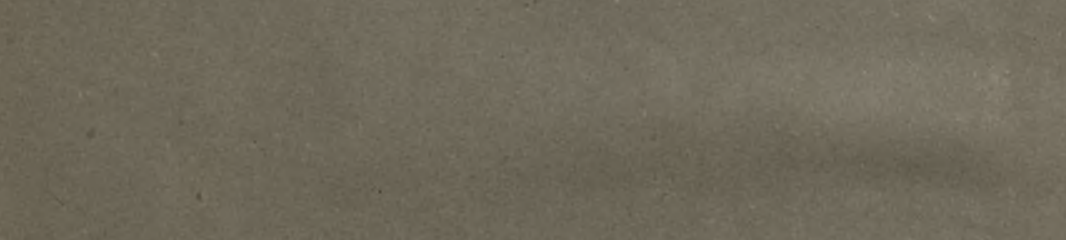

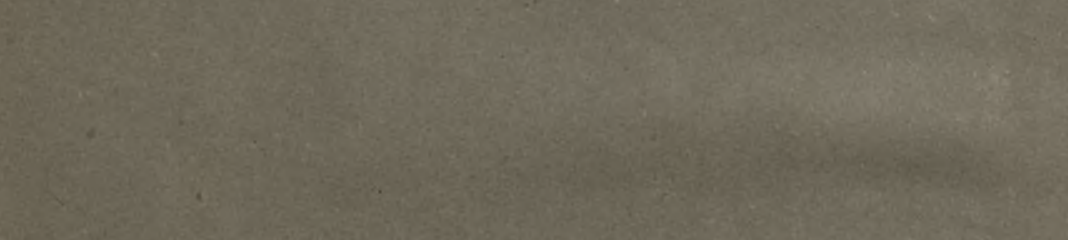

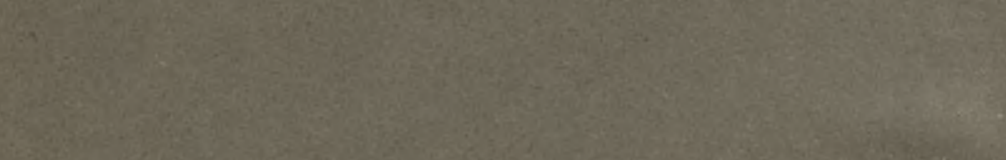

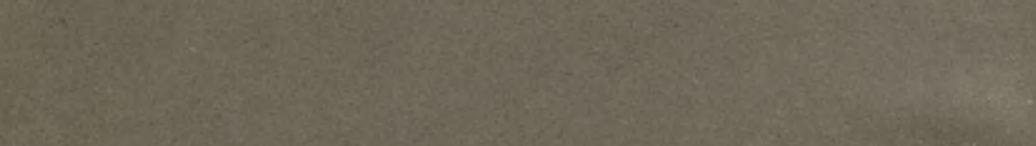

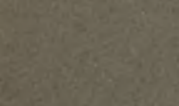
2.

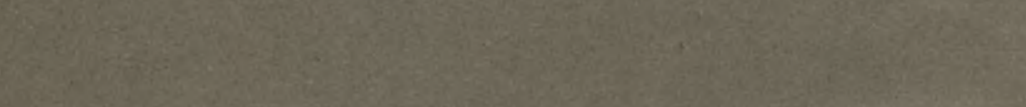

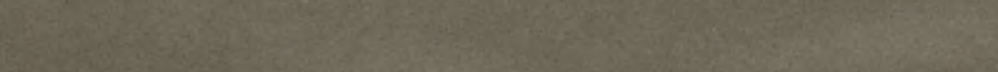

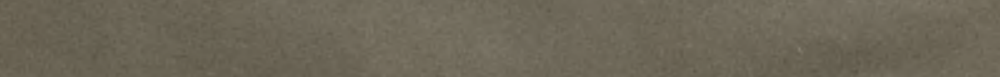

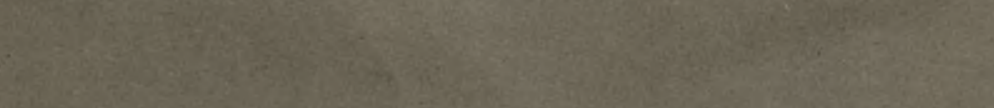

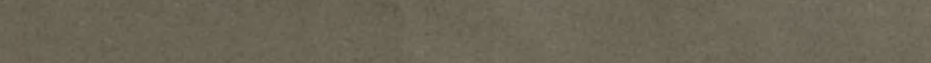

12.

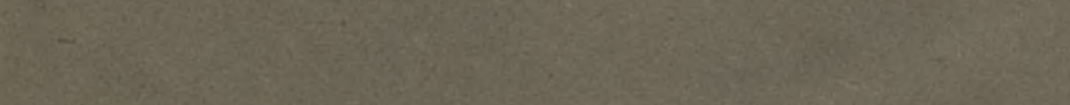




\title{
Grundzüge und Aufgaben
}

der

\section{Differential- und Integralrechnung}

\author{
nebst den Resultaten
}

von

\section{Dr. H. Dölp,}

weiland Professor am Polytechnikum zu Darmstadt

neu bearbeitet

von

Dr. Eugen Netto,

Professor der Mathematik an der Universität zu Giessen

12. A nflage

Alfred Töpelmann

(vormals J. Ricker'sche Verlagsbuchhandlung)

Giessen 1909 


\section{kb $517.2 \% .3$}

1. Auflage: 1869

2. $" 1874$

$3 . \quad 1878$

4. $\quad 1885$

5.

6. $\quad 1895$

$7 . \quad 1898$

8. $\quad 1900$

$9 . \quad 1901$

$10 . \quad 1903$

11. Doppelauflage: 1905

$12 . \quad 1909$

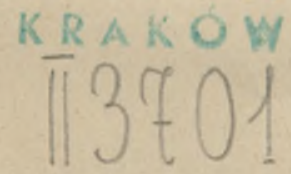




\section{Vorwort zur elften Auflage.}

Dem Texte nach unverändert, dem Titel nach verändert erscheint die neue, elfte Auflage des Dölp'schen Werkes. Beides hat seine guten Gründe. Die für das Erste sind technischer Natur; es erübrigt, auf sie einzugehen. Die für das Zweite liegen in der Verschiedenheit des Charakters der früheren und der letzten Auflagen; eine Verschiedenheit, die sich allmählich herausgebildet hat und nun endlich, ihren Einfluss auszuüben, für geboten hält. Die „zur Lösung der Aufgaben nötigen theoretischen Erläuterungen ", wie es bisher hiess, haben sich zu "Grundzügen der Differential- und Integral-Rechnung “ ausgewachsen; diese beanspruchen eine selbständigere Stellung und gedenken sie in Zukunft noch weiter zu befestigen. Hierdurch ist zugleich der Kreis für die Verwendbarkeit des Buches stark erweitert worden, zumal der Verleger in eine bedeutende Herabsetzung des Preises eingewilligt hat. Somit erscheint die Hoffnung berechtigt, dass das Werk auch in Zukunft bei dem Studium der Infinitesimal-Rechnung Nutzen und Hilfe gewährt.

Giessen, im November 1905.

\section{E. Netto.}


Das INHALTSVERZEICHNIS

befindet sich am Schlusse des Buches 


\section{Differentialrechnung.}

\section{Funktionen einer unabhängigen Variabelen.}

\section{§ 1. Zusammenstellung der Differentialquotienten der einfachen Funktionen.}

$y=a x^{n}$

$y=\frac{a}{x^{n}}=a x^{-n}$

$y=a \sqrt[n]{x}=a x^{\frac{1}{n}}$

$y=a \sqrt[n]{x^{p}}=a x^{\frac{p}{n}}$

$y=\sqrt{x}$

$y=e^{x}$

$y=a^{x}$

$y=\lg x$

$y=\sin x$

$y=\cos x$

$y=\operatorname{tg} x$

$y=\operatorname{cotg} x$

$y=\arcsin x$

$$
\frac{d y}{d x}=n a x^{n-1}
$$$$
n=-\frac{n a}{x^{n+1}}=-n a x^{-n-1}
$$$$
"=\frac{a}{n} \sqrt[n]{x^{1-n}}=\frac{1}{n} a x^{\frac{1}{n}-1}
$$$$
n=\frac{p}{n} a \sqrt[n]{x^{p-n}}=\frac{p}{n} a x^{\frac{p}{n}-1}
$$$$
n=\frac{1}{2 \sqrt{x}}
$$$$
\text { " }=e^{x}
$$$$
"=a^{x} \lg a
$$$$
n=\frac{1}{x}
$$$$
n=\cos x
$$$$
n=-\sin x
$$$$
"=\frac{1}{\cos ^{2} x}
$$$$
n=-\frac{1}{\sin ^{2} x}
$$$$
n=\frac{1}{\sqrt{1-x^{2}}}
$$ 
$y=\arccos x$

$$
\begin{aligned}
\frac{d y}{d x} & =-\frac{1}{\sqrt{1-x^{2}}} \\
n & =\frac{1}{1+x^{2}}
\end{aligned}
$$

$y=\operatorname{arctg} x$

$y=\operatorname{arccotg} x$

$$
n=-\frac{1}{1+x^{2}} \text {. }
$$

§ 2. Erklärung der Funktion einer unabhängigen Variabelen. Erklärung und Entwicklung des Differentialquotienten.

Eine Gleichung, in welcher eine einzige unbestimmte Grösse $x$ mit einer beliebigen Anzahl von gegebenen und unverändert beibehaltenen (k onstanten) Grössen $a, b, c, \ldots$ durch irgend welche Rechnungsoperationen verbunden auftritt, ist entweder eine identische Gleichung oder eine $\mathrm{Be}$ stimmungs-Gleichung. Eine identische Gleichung ist eine solche, die für jeden Wert der Grösse $x$ gilt, wie z. B.

$$
(x+a)^{2}=x^{2}+2 a x+a^{2} \text {. }
$$

Eine Bestimmungs-Gleichung ist eine solche, die nicht für jeden Wert von $x$ gültig bleibt; ein Beispiel hierfür bietet

$$
x^{2}-2 a x+b=0 .
$$

Bei einer Bestimmungs-Gleichung entsteht daher die Aufgabe, denjenigen Wert, oder diejenigen Werte von $x \mathrm{zu}$ finden, welche die Gleichung erfüllen. Die Grösse $x$ heisst deshalb die Unbekannte. Ihre Bestimmung ist Aufgabe der Theorie der Gleichungen.

Eine Gleichung, in welcher ausser den gegebenen Konstanten zwei unbestimmte Grössen $x$ und $y$ auftreten, kann ebenfalls eine identische Gleichung sein, wie z. B.

$$
(x+y)(x-y)=x^{2}-y^{2} .
$$

Ist jedoch die Gleichung keine identische, dann kann sie zu einer Bestimmungs-Gleichung für die eine der beiden Grössen $y$ (oder $x$ ) dadurch gemacht werden, dass man der anderen $x$ (oder $y$ ) irgend einen bestimmten Wert beilegt. Dieser Wert kann beliebig gewählt werden; von ihm ist die andere Grösse 
abhängig. Die beliebig gewählte Grösse $x$ (oder $y$ ) heisst deshalb die unabhängige Variabele; die andere durch sie bestimmte $y$ (oder $x$ ) heisst die abhängige Variabele; sie wird auch als Funktion der unabhängigen Variabelen bezeichnet.

1. Beispiel: (a) $A x+B y+C=0$,

folglich (b) $y=-\frac{A x+C}{B}$,

oder auch (c) $x=-\frac{B y+C}{A}$.

2. Beispiel: (a) $\frac{x^{2}}{a^{2}}+\frac{y^{2}}{b^{2}}-1=0$,

folglich (b) $y= \pm \frac{b}{a} \sqrt{a^{2}-x^{2}}$,

oder auch (c) $x= \pm \frac{a}{b} \sqrt{b^{2}-y^{2}}$.

In beiden Beispielen ist der Zusammenhang der Variabelen durch jede der Gleichungen (a), (b), (c) ausgedrückt. In (b) ist die Variabele $y$ als analytischer Ausdruck dargestellt, der ausser den konstanten Grössen nur die unabhängige Variabele $x$ enthält; man sagt hierbei: $y$ ist explicite als Funktion von $x$ gegeben, oder, was dasselbe bedeutet: $y$ ist eine entwickelte Funktion von $x$. Ebenso liefert (c) die Variabele $x$ als entwickelte Funktion von $y$. Die Darstellungen (a) geben dagegen die eine der beiden Variabelen durch die andere in impliciter oder unentwickelter Form. Symbole wie die folgenden:

$$
y=f(x), y=\varphi(x), y=F(x), \ldots
$$

dienen zur allgemeinen Bezeichnung expliciter Funktionen von $x$; die mit $x$ verbundenen Konstanten sind hier nicht besonders hervorgehoben. In gleicher Weise kann man

$$
f(x, y)=0, g(x, y)=0, h(x, y)=0, \ldots
$$

als Symbole für das implicite Abhängigkeitsverhältnis ansehen, welches zwischen den Variabelen $x$ und $y$ besteht. 
Es ist eine Eigentümlichkeit vieler Funktionen, dass jedem beliebigen Werte von $x$ auch nur ein einziger Wert von $y$ entspricht, wie in $y=x^{2}, y=x^{8}, y=\sin x$; doch giebt es auch solche, die zu jedem Werte von $x$ mehr als einen Wert von $y$ liefern, wie $y=\sqrt{x}, y=\sqrt[3]{x}, y=\operatorname{arc} \sin x$. Hiernach werden ein- und mehrwertig e Funktionen unterschieden; die mehrwertigen können zwei-, drei-, ... wertige, ja auch wie $y=\operatorname{arc} \sin x$ unendlich vielwertige sein.

Substituiert man in einer entwickelten Funktion $y=f(x)$ an Stelle der unabhängigen Variabelen $x$ die beiden verschiedenen Werte $x$ und $x_{1}$, so ergeben sich die entsprechenden Funktionswerte $y=f(x)$ und $y_{1}=f\left(x_{1}\right)$, und man kann aus beiden Wertpaaren den folgenden Differenzenquotienten zusammensetzen:

$$
\frac{y_{1}-y}{x_{1}-x}=\frac{f\left(x_{1}\right)-f(x)}{x_{1}-x} .
$$

Wir bezeichnen die Differenzen

$$
x_{1}-x=\Delta x, y_{1}-y=f\left(x_{1}\right)-f(x)=\Delta y=\Delta f(x),
$$

so dass (1) auch geschrieben werden kann:

$$
\frac{\Delta y}{\Delta x}=\frac{\Delta f(x)}{\Delta x}=\frac{f(x+\Delta x)-f(x)}{\Delta x} .
$$

Der Wert des Differenzenquotienten liefert das Verhältnis des Zuwachses der abhängigen zum Zuwachs der unabhängigen Veränderlichen.

Wir wollen den Differenzenquotienten auch geometrisch repräsentieren. $\mathrm{Zu}$ diesem $\mathrm{Zwecke}$ erinnern wir an die aus der analytischen Geometrie her bekannte Darstellung der Funktion $y=f(x)$ durch eine Kurve in der $X Y$-Ebene. Die Abscisse $O Q$ eines Kurvenpunktes $P$ giebt den Wert der Veränderlichen $x$ und seine Ordinate $Q P$ den Wert der Funktion $f(x)$ an. Je nachdem $f(x)$ ein- oder mehrwertig ist, gehören zu der Abscisse $x$ eine oder mehrere Ordinaten $y$. Der Punkt $P$ der Kurve möge die Abscisse $x$ und die Ordinate $y$ besitzen. Wir schreiben dies kurz $P=(x, y)$. Ebenso sei 
$P_{1}=\left(x_{1}, y_{1}\right)=(x+\Delta x, y+\Delta y)$. Dann ist $P R=Q Q_{1}$ $=x_{1}-x=\Delta x$ und $P_{1} R=y_{1}-y=\Delta y$, und wir erhalten

$$
\frac{\Delta y}{\Delta x}=\frac{f(x+\Delta x)-f(x)}{\Delta x}=\operatorname{tang} \beta .
$$

Wir können $\operatorname{tang} \beta$ in einfacher und verständlicher Art das Steigungsmass der Geraden $S P P_{1}$ nennen. Dann sieht man:

Fig. 1.

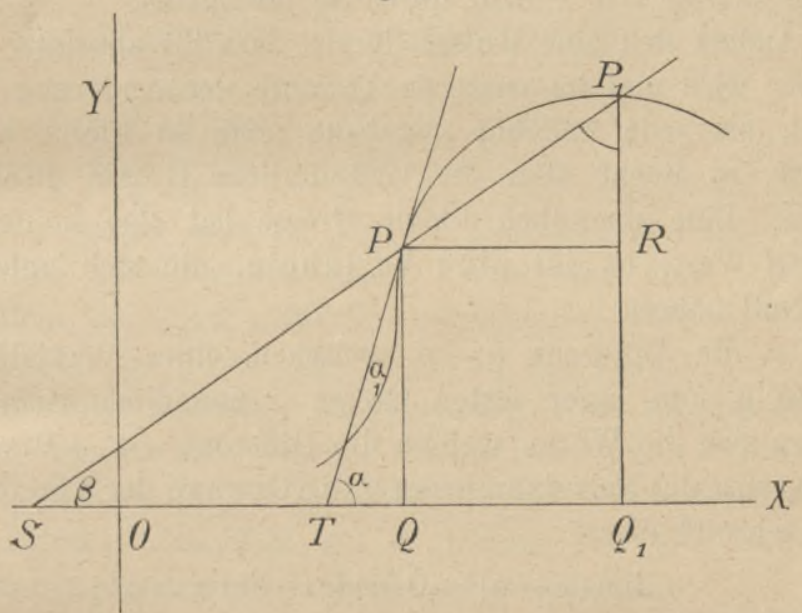

Der Differenzenquotient (1) oder (1 $\left.{ }^{a}\right)$ giebt das Steigungsmass der Sekante $P P_{1}$ an, welche zu den Kurvenpunkten $P=(x, y)$ und $P_{1}=(x+\Delta x, y+\Delta y)$ gehört.

Da wir die Funktion $f$ als gegeben voraussetzen, so kann in jedem einzelnen Falle dieser Quotient auch ausgerechnet werden.

1. Beispiel: $y=a x^{2}, y_{1}=a x_{1}^{2}$;

$$
\frac{\Delta y}{\Delta x}=\frac{y_{1}-y}{x_{1}-x}=a \frac{x_{1}^{2}-x^{2}}{x_{1}-x}=a\left(x_{1}+x\right) .
$$

2. Beispiel: $y=\sqrt{x}, y_{1}=\sqrt{x_{1}}$;

$$
\frac{\Delta y}{\Delta x}=\frac{y_{1}-y}{x_{1}-x}=\frac{\sqrt{x_{1}}-\sqrt{x}}{x_{1}-x}=\frac{1}{\sqrt{x_{1}}+\sqrt{x}} .
$$


Wir wollen nun in der Figur den Punkt $P_{1}$ auf der Kurve unbegrenzt dem als fest angenommenen Punkte $P$ sich nähern lassen. Um richtige analytische Einsicht in diesen Prozess zu gewinnen, müssen wir einige neue Begriffe einführen.

Sieht man bei einer reellen Grösse $a$ von dem Vorzeichen ab, so spricht man von ihrem absoluten Betrage; beispielsweise haben +1 und -1 den absoluten Betrag 1 . Der absolute Betrag von $a$ wird durch $|a|$ bezeichnet.

Ändert sich eine Grösse derart, dass ihr absoluter Betrag kleiner wird und im weiteren Verlaufe der Änderung kleiner bleibt, als jede beliebig gegebene noch so kleine absolute Grösse, so nennt man die veränderliche Grösse unendlich klein. Eine unendlich kleine Grösse hat also keinen konstanten Wert; es ist eine Variabele, die sich unbegrenzt der Null nähert.

Ist die Differenz $\alpha-a$ zwisehen einer veränderlichen Grösse $\alpha$ und einer festen Grösse $a$ unendlich klein, d. h. nähern sich die Werte, welche die Differenz $\alpha-a$ durchläuft, unbegrenzt der Null, dann heisst $a$ die Grenze der Variabelen $\alpha$. Man schreibt dies

$$
\lim (\alpha-a)=0 \quad \text { oder } \quad \lim \alpha=a
$$

wobei "lim" das Wort „limes", "Grenze" bezeichnet. Durchläuft etwa $\alpha$ eine der Reihen

$$
\begin{aligned}
& 1, \frac{1}{2}, \frac{1}{3}, \frac{1}{4}, \ldots \frac{1}{n}, \frac{1}{n+1}, \ldots \\
& \frac{3}{2}, \frac{3}{4}, \frac{9}{8}, \frac{15}{16}, \ldots \frac{2^{2 n}-1}{2^{2 n}}, \frac{2^{2 n+1}+1}{2^{2 n+1}}, \ldots
\end{aligned}
$$

so ist im ersten Falle $\lim \alpha=0$, im zweiten $\lim \alpha=1$. Das Zeichen "lim" wendet man in uneigentlichem Sinne auch für den Fall an, dass die Grösse $\alpha$ tiber alle Grenzen hinaus wächst; man sohreibt $\operatorname{dann} \lim \alpha=\infty$, trotzdem ja von einer Grenze keine Rede sein kann.

Ist $\lim \alpha=a$ und $\lim \beta=b$, so folgt daraus

$$
\lim (\alpha \pm \beta)=a \pm b, \lim (\alpha \beta)=a b,
$$


weil mit $\alpha-a$ und $\beta-b$ zugleich auch

$$
\begin{gathered}
(\alpha \pm \beta)-(a \pm b) \text { und } \\
(\alpha-a)(\beta+b)+(\alpha+a)(\beta-b)=2(\alpha \beta-a b)
\end{gathered}
$$

unendlich klein werden. Ferner ist unter der Voraussetzung, dass $b$ von Null verschieden sei,

$$
\lim \frac{\alpha}{\beta}=\frac{a}{b}
$$

denn mit $\alpha-a$ und $\beta-b$ wird dann zugleich auch

unendlich klein.

$$
\frac{(\alpha-a) b-(\beta-b) a}{\beta b}=\frac{a b-\beta a}{\beta b}=\frac{\alpha}{\beta}-\frac{a}{b}
$$

Wir wollen nun in $\left(1^{\mathrm{a}}\right)$ die Differenz $\Delta x$ bei festem $x$ unendlich klein werden lassen. Wird dann auch $\Delta y$ unendlich klein, so heisst $y$ für den betrachteten Wert von $x$ stetig variabel. Ist $y$ für alle Werte $x$, die zwischen $x_{1}$ und $x_{2}$ liegen, stetig variabel, so sagt man, $y$ sei für das Intervall von $x_{1}$ bis $x_{2}$ eine stetige Funktion von $x$. Ob für unendlich kleine $\Delta x$ der Quotient $\left(1^{\mathrm{a}}\right)$ sich einer Grenze nähert, ist fraglich. Geschieht es, so nennen wir sie den Differentialquotienten von $y$ für $x$ und schreiben

$$
\frac{d y}{d x}=y^{\prime}=f^{\prime}(x)=\lim \frac{\Delta y}{\Delta x}=\lim \frac{f(x+\Delta x)-f(x)}{\Delta x},
$$

wobei die Bezeichnungen $\frac{d y}{d x}, y^{\prime}, f^{\prime}(x)$ für einander eintreten können und vollkommen gleichbedeutend sind. Dass der Differentialquotient, der ja die Grenze des Quotienten zweier unendlich kleiner Grössen ist, doch einen bestimmten endlichen Wert haben kann, zeigen schon unsere obigen Beispiele. Für

$$
y=a x^{2} \text { wird } \frac{d y}{d x}=y^{\prime}=\lim a(x+x+\Delta x)=2 a x,
$$

und für

$$
y=\sqrt{x} \text { wird } \frac{d y}{d x}=y^{\prime}=\lim \frac{1}{\sqrt{x}+\sqrt{x+\Delta x}}=\frac{1}{2 \sqrt{x}} .
$$


Bei der Schreibweise $\frac{d y}{d x}$ ist streng daran festzuhalten, dass Zähler und Nenner nicht für sich einzeln definiert worden sind, und dass $d x, d y$ nicht die Bedeutung von $\lim \Delta x$, $\lim \Delta y$ haben. Trotzdem dürfen wir uns erlauben, in unseren Beispielen $d y=2 a x d x$ oder $d y=\frac{1}{2 \sqrt{x}} d x$ und allgemein

$$
d y=y^{\prime} d x=f^{\prime}(x) d x=f(x+d x)-f(x)
$$

zu schreiben, sofern wir darunter eben weiter nichts verstehen, als die Beziehungen (2). Irrtümer können dadurch nicht hervorgerufen werden.

Wie der Differenzenquotient, so lässt auch der Differentialquotient eine einfache geometrische Deutung zu. Wir lassen in der Figur 1 die Punkte $P$ und $Q$ fest und nähern den Punkt $Q_{1}$ unbegrenzt dem Punkte $Q$. Dabei rïckt der Punkt $P_{1}$ der Kurve unbegrenzt an den Punkt $P$ heran und die Tangente $T P$ wird Grenzlage der Sekante $S P$. Der Differentialquotient stellt das Steigungsmass der Tangente im Punkte $P=(x, y)$ dar:

$$
\tan g \alpha=\lim \frac{\Delta y}{\Delta x}=\frac{d y}{d x}=y^{\prime}=f^{\prime}(x) .
$$

Nach diesen vorbereitenden Erörterungen ist es uns möglich geworden, die Formeln zu entwickeln, nach welchen aus den einzelnen Funktionsformen der zugehörige Differentialquotient abgeleitet werden kann. Wir beginnen mit

$$
y=f(x)=a x_{1}^{n}(n \text { ganz und positiv). }
$$

Den Werten $x$ und $x_{1}$ entsprechen die Funktionswerte $y=a x^{n}$ und $y_{1}=a x_{1}^{n}$ und daraus entsteht: $\frac{d y}{d x}=\lim a\left(\frac{x_{1}^{n}-x^{n}}{x_{1}-x}\right)=\lim a\left(x_{1}{ }^{n-1}+x_{1}{ }^{n-2} x+\ldots+x^{n-1}\right)$

Wird nun $x_{1}=x$, so erhält man

$$
\frac{d y}{d x}=n a x^{n-1}
$$


Wird $n=0$, so ist $y=a$, d. h. eine Konstante, und daher auch $y_{1}=a$. Die Differenz $\Delta y$ ist mithin gleich Null, und ebenso ist der Differenzenquotient $\Delta y: \Delta x=0$. Geht man zur Grenze über, so folgt für

$$
y=a=\text { Konstante }, \quad \frac{d y}{d x}=0 .
$$

Die Richtigkeit dieser Formel lässt sich auch an der Funktionskurve nachweisen. Sie geht für $y=a$ in eine zur $X$-Axe parallele gerade Linie uber, deren Tangente in jedem Punkte mit ihr zusammenfällt. Deshalb ist $\alpha=0, \tan g \alpha=0$.

Wir zeigen zunächst, dass (4) auch für negative ganze Zahlen $n$ gilt. Wir setzen:

$$
y=\frac{a}{x^{n}}=a x^{-n} \text { ( } n \text { ganz und positiv) }
$$

und finden ähnlich wie oben

$$
\begin{aligned}
\frac{d y}{d x} & =\lim a \frac{\frac{1}{x_{1}^{n}}-\frac{1}{x^{n}}}{x_{1}-x}=\lim \frac{-a}{x_{1}^{n} x^{n}} \cdot \frac{x_{1}{ }^{n}-x^{n}}{x_{1}-x} \\
& =\lim \frac{-a}{x_{1}^{n} x^{n}}\left(x_{1}{ }^{n-1}+x_{1}{ }^{n-2} x+\ldots+x^{n-1}\right) .
\end{aligned}
$$

Wird nun $x_{1}=x$, so erhält man, einem negativen $n$ in (4) entsprechend:

$$
\frac{d y}{d x}=-n a x^{-n-1} \text {. }
$$

Um den Gültigkeitsbereich von (4) noch zu erweitern, fuhren wir den Begriff der umgekehrten Funktion ein. $\mathrm{Zu}$ jeder Funktion $y=f(x)$ ist eine andere denkbar $x=\varphi(y)$, die so beschaffen ist, dass jedes Wertepaar $(x, y)$, welches die eine Gleichung befriedigt, auch der andern genüge leistet. $f(x)$ und $\varphi(y)$ stehen zu einander in dem Verhältnisse, wie die Funktionen $(b)$ und $(c)$ in den beiden Beispielen auf Seite 3. Man erkennt leicht, wie man aus der, die Funktion $y=f(x)$ darstellenden Kurve zu der $y=\varphi(x)$ darstellenden gelangen kann. Dazu ist es nur nötig, die ganze erste Figur um die Halbierungslinie des Winkels zwischen den beiden positiven Halbaxen umzuklappen. 
Sind $(x, y)$ und $(x+\Delta x, y+\Delta y)$ zwei Wertepaare welche $y=f(x)$ und $x=\varphi(y)$ befriedigen, so ist identisch:

$$
\frac{\Delta y}{\Delta x} \cdot \frac{\Delta x}{\Delta y}=1 \text {. }
$$

Da die Grenze eines Produkts gleich dem Produkte der Grenzen ist, so folgt hieraus:

$$
\frac{d y}{d x} \cdot \frac{d x}{d y}=f^{\prime}(x) \cdot \varphi^{\prime}(y)=y^{\prime} \cdot x^{\prime}=1,
$$

d. h. ist eine Funktion die Umkehrung einer anderen, so ist das Produkt aus ihren beiden Differentialquotienten gleich der Einheit.

Auch die Funktionskurve lässt die Richtigkeit von leicht erkennen, da bei dem Umklappen die Winkel $\beta$ und $\alpha$ durch $\beta_{1}=\frac{\pi}{2}-\beta$ und $\alpha_{1}=\frac{\pi}{2}-\alpha$ zu ersetzen sind; deshalb wird:

$$
\frac{d y}{d x} \cdot \frac{d x}{d y}=\tan g \alpha \cdot \tan g \alpha_{1}=1 .
$$

Mit Hülfe von (5) kann jetzt nachgewiesen werden, dass die Funktion:

$$
y=a \sqrt[n]{x}=a x^{\frac{1}{n}} \quad(n \text { ganz })
$$

ebenfalls nach (4) differentiiert werden kann. Der umgekehrten Funktion $x=\frac{1}{a^{n}} y^{n}$ entspricht nämlich der Differentialquotient

$$
\frac{d x}{d y}=\frac{1}{a^{n}} n y^{n-1}=\frac{1}{a} n x^{1-\frac{1}{n}},
$$

und daraus geht nach (5), übereinstimmend mit (4), hervor:

$$
\frac{d y}{d x}=\frac{1}{n} \cdot a x^{\frac{1}{n}-1} \text {. }
$$

Ist speciell $y=\sqrt{x}$, so wird $\frac{d y}{d x}=\frac{1}{2 \sqrt{x}}$.

Funktion einer Funktion. Wenn $y$ eine Funktion von $z$ ist, etwa $y=f(z)$, und $z$ selbst wieder eine Funktion 
von $x$, etwa $z=g(x)$, so folgt aus $y=f[g(x)]$, dass $y$ auch eine Funktion von $x$ ist. $\mathrm{Zu}$ den Werten $x, x_{1}$ mögen gemäss der Funktionsbeziehungen die Werte $y, y_{1}$ und $z, z_{1}$ gehören. Wir setzen:

$$
x_{1}-x=\Delta x, \quad y_{1}-y=\Delta y, \quad z_{1}-z=\Delta z,
$$

dann ist identisch:

$$
\frac{\Delta y}{\Delta x}=\frac{\Delta y}{\Delta z} \cdot \frac{\Delta z}{\Delta x}
$$

und nach dem Satze von der Grenze eines Produktes:

$$
\frac{d y}{d x}=\frac{d y}{d z} \cdot \frac{d z}{d x}
$$

Ist $y$ eine Funktion $v$ on $z$, und $z$ selbst eine Funktion von $x$, so ist der Differentialquotient von $y$ nach $x$ gleich dem Produkte aus dem Differentialquotienten von $y$ nach $z$ in den Differentialquotienten von $z$ nach $x$.

Beispiel: $y=(a x)^{2}=z^{2}$, wenn $z=a x$ gesetzt wird;

$$
\frac{d y}{d z}=2 z, \quad \frac{d z}{d x}=a ; \quad \frac{d y}{d x}=2 z \cdot a=2 a^{2} x .
$$

Aus $y=a^{2} x^{2}$ erhält man denselben Wert.

Die Formel (6) erweitert sich dadurch, dass wir annehmen, es sei:

$$
y=f(u), u=\varphi(z), z=\varrho(x),
$$

indem man alsdann hat:

$$
\frac{d y}{d x}=\frac{d y}{d u} \cdot \frac{d u}{d x}=\frac{d y}{d u} \cdot \frac{d u}{d z} \cdot \frac{d z}{d x} .
$$

Jetzt ist es endlich möglich geworden, die Anwendbarkeit der Formel (4) auch für den Fall nachzuweisen, dass der Exponent ein Bruch, und die Funktion also von der Form ist:

$$
y=a \sqrt[n]{x^{p}}=a x^{\frac{p}{n}} .
$$

Setzt man nämlich $z=x^{p}$, so wird

$$
y=a z^{\frac{1}{n}}, z=x^{p} ; \frac{d y}{d z}=\frac{1}{n} a z^{\frac{1}{n}-1}, \frac{d z}{d x}=p x^{p-1}
$$




$$
\frac{d y}{d x}=\frac{1}{n} a\left(x^{p}\right)^{\frac{1}{n}-1} \cdot p x^{p-1}=\frac{p}{n} a x^{\frac{p}{n}-1} .
$$

Hiermit ist die Gültigkeit der Formel (4) für alle rationalen Werte des Exponenten nachgewiesen, und wir können sagen:

Eine Potenz der Variabelen mit rationalem Exponenten wird differentiirt,.indem man ihren Koeffizienten mit dem Exponenten multipliziert und diesen selbst um die Einheit vermindert.

1. Beispiel. $f(x)=4 x^{3}, \quad f^{\prime}(x)=12 x^{2}$.

2. Beispiel. $f(x)=2 x^{-8}, f^{\prime}(x)=-6 x^{-4}$.

3. Beispiel. $f(x)=4 x^{\frac{3}{2}}, \quad f^{\prime}(x)=6 x^{\frac{1}{2}}$.

4. Beispiel. $f(x)=2 x^{-\frac{1}{2}}, f^{\prime}(x)=-x^{-\frac{3}{2}}$.

Diezusammengesetzten Funktionen. Werden mehrere Funktionen durch irgend welche Rechnungsoperationen mit einander verbunden, so heisst eine solche Verbindung eine zusammengesetzte Funktion. Sind zwei derartige Funktionen wie $u=\varphi(x), v=\psi(x)$ zur Summe oder Differenz $y=u \pm v$ verbunden, so ist $\Delta y=\Delta u \pm \Delta v$ und daraus folgt durch Division mit $\Delta x$ und Grenzübergang:

$$
\frac{d y}{d x}=\frac{d u}{d x} \pm \frac{d v}{d x} \quad \text { oder } \quad(u \pm v)^{\prime}=u^{\prime} \pm v^{\prime} .
$$

Zur Erweiterung setzen wir $v \pm w$ statt $v$ und finden sofort: $\frac{d y}{d x}=\frac{d u}{d x} \pm \frac{d v}{d x} \pm \frac{d w}{d x} \quad$ oder $\quad(u \pm v \pm w)^{\prime}=u^{\prime} \pm v^{\prime} \pm w^{\prime}$.

Der Differentialquotient einer Summe von Funktionen der nämlichen Variabelen ist gleich der Summe aus den Differentialquotienten der einzelnen Funktionen. Der Differentialquotient aus einer Differenz zweier Funktionen der nämlichen Variabelen ist gleich der Differenz aus den Differentialquotienten von Minuendus und Subtrahendus.

Für das Produkt $y=u, v$ ergeben sich folgende Beziehungen: 


$$
\begin{aligned}
y+\Delta y & =(u+\Delta u)(v+\Delta v) \\
\Delta y & =u \Delta v+v \Delta u+\Delta u \Delta v \\
\frac{\Delta y}{\Delta x} & =u \frac{\Delta v}{\Delta x}+v \frac{\Delta u}{\Delta x}+\frac{\Delta u}{\Delta x} \frac{\Delta v}{\Delta x} \Delta x
\end{aligned}
$$

$\lim \frac{\Delta y}{\Delta x}=u \lim \frac{\Delta v}{\Delta x}+v \lim \frac{\Delta u}{\Delta x}+\lim \frac{\Delta u}{\Delta x} \lim \frac{\Delta v}{\Delta x} \lim \Delta x ;$

da das letzte Glied der letzten Gleichung verschwindet, so hat man

$$
\frac{d y}{d x}=u \frac{d v}{d x}+v \frac{d u}{d x} \quad \text { oder } \quad(u \cdot v)^{\prime}=u v^{\prime}+v u^{\prime} .
$$

Ein Produkt aus zwei Funktionen der nämlichen Variabelen wird differentiiert, indem man den Differentialquotienten des ersten Faktors mit dem zweiten Faktor und den Differentialquotienten des zweiten Faktors mit dem ersten Faktor multipliziert und beide Produkte addiert.

1. Beispiel: $y=x^{7} \cdot x^{4} u \cdot v ; u^{\prime}=3 x^{2}, v^{\prime}=4 x^{3}$, $y^{\prime}=x^{4} \cdot 3 x^{2}+x^{8} \cdot 4 x^{3}=7 x^{6}$, ein Wert, welcher auch aus der direkten Form $y=x^{7}$ hervorgeht.

2. Beispiel: $y=\left(a+b x^{2}\right)\left(c+e x^{3}\right)=u . v ; \quad u^{\prime}=2 b x$, $v^{\prime}=3 e x^{2} ; \quad y^{\prime}=2 b x\left(c+e x^{3}\right)+3 e x^{2}\left(a+b x^{2}\right)=2 b c x$ $+3 a e x^{2}+5 b e x^{4}$. Das gleiche Resultat wird erhalten, wenn man zuerst die Multiplikation ausführt und dann differentiiert.

Geht $v$ in $v w$ über, dann verwandelt $\operatorname{sich} v^{\prime}$ in $v^{\prime} w+w^{\prime} v$ und wir erhalten statt (8):

$$
y^{\prime}=(u v w)^{\prime}=v w \cdot u^{\prime}+u w \cdot v^{\prime}+u v \cdot w^{\prime} \text {. }
$$

Tritt an die Stelle des veränderlichen Faktors $v$ die Konstante $a$, so ist $v^{\prime}=0$ und daher wird für $y=a u$ jetzt $y^{\prime}=a u^{\prime}$ oder $(a u)^{\prime}=a u^{\prime}$.

Der Differentialquotient eines Produkts aus einer Konstanten mit einer Funktion von $x$ ist gleich dem Produkt aus dieser Konstanten und dem Differentialquotienten der Funktion. 
Für den Quotienten $y=\frac{u}{v}$ folgt:

$$
\begin{aligned}
y+\Delta y & =\frac{u+\Delta u}{v+\Delta v}, \\
\Delta y & =\frac{u+\Delta u}{v+\Delta v}-\frac{u}{v}=\frac{v \Delta u-u \Delta v}{v(v+\Delta v)}, \\
\frac{\Delta y}{\Delta x} & =\frac{v \frac{\Delta u}{\Delta x}-u \frac{\Delta v}{\Delta x}}{v(v+\Delta v)} \\
\lim \frac{\Delta y}{\Delta x} & =\frac{v \lim \frac{\Delta u}{\Delta x}-u \lim \frac{\Delta v}{\Delta x}}{v(v+\lim \Delta v)} \\
\frac{d y}{d x} & =\frac{v \cdot u^{\prime}-u v^{\prime}}{v^{2}} .
\end{aligned}
$$

Der Differentialquotient eines Bruches ist gleich dem Differentialquotienten des Zählers multipliziert mit dem Nenner, weniger dem Differentialquotienten des Nenners multipliziert mit dem Zähler, das Ganze geteilt durch das Quadrat des Nenners.

$$
\text { Beispiel: } \begin{aligned}
y & =\frac{x^{2}-a^{2}}{x-a}=\frac{u}{v} ; u^{\prime}=2 x, v^{\prime}=1, \\
y^{\prime} & =\frac{2 x(x-a)-1 \cdot\left(x^{2}-a^{2}\right)}{(x-a)^{2}}=1 .
\end{aligned}
$$

Aus der reduzierten Form $y=x+a$ entsteht derselbe Wert. Einfacher kann der Differentialquotient eines Quotienten aus demjenigen eines Produkts hergeleitet werden. Ist nämlich

$$
\begin{aligned}
& y=\frac{u}{v}, \text { so ist } u=y \cdot v, u^{\prime}=v y^{\prime}+y v^{\prime}, \\
& y^{\prime}=\frac{u^{\prime}-y v^{\prime}}{v}=\frac{u^{\prime}-\frac{u}{v} \cdot v^{\prime}}{v}=\frac{u^{\prime} v-u v^{\prime}}{v^{2}} .
\end{aligned}
$$

Die abgeleiteten Theoreme über die Differentiation zusanımengesetzter Funktionen sind besondere Fälle eines allgemeinen Satzes, zu dessen Herleitung wir nun tibergehen wollen, nachdem noch eine neue Bezeichnung eingeführt worden ist. 
Bedeutet $f(u, v)$ eine Funktion zweier Variabelen $u, v$, so kann die Differentiation entweder nach $u$ vor sich gehen, wobei $v$ als konstant angesehen wird, oder umgekehrt nach $v$, wobei $u$ als konstant gilt. Solche Differentiation nach einer der beiden Variabelen heisst partielle Differentiation, und ihr Resultat ist der partieller Differentialquotient nach $u$ oder nach $v$. Um darauf hinzudeuten, unterscheidet man diese Operation durch Benutzung eines runden $\partial$ von dem totalen Differentiieren und schreibt

$$
\begin{aligned}
& \frac{\partial f(u, v)}{\partial u}=\lim \frac{f(u+\Delta u, v)-f(u, v)}{\Delta u}, \\
& \frac{\partial f(u, v)}{\partial v}=\lim \frac{f(u, v+\Delta v)-f(u, v)}{\Delta v} .
\end{aligned}
$$

Wir gehen jetzt zu dem angekündigten Satze tiber. Es sei $y=f(u, v)$ und hierbei $u$ und $v$ Funktionen von $x$, etwa $u=\varphi(x)$ und $v=\psi(x)$. Es soll $\frac{d y}{d x}$ bestimmt werden. Zu den Werten $x$ und $x_{1}$ möge $u, v, y$ und $u_{1}, v_{1}, y_{1}$ gehören. Dann ist

$$
\begin{aligned}
\frac{y_{1}-y}{x_{1}-x} & =\frac{f\left(u_{1}, v_{1}\right)-f(u, v)}{x_{1}-x} \\
& =\frac{f\left(u_{1}, v_{1}\right)-f\left(u, v_{1}\right)+f\left(u, v_{1}\right)-f(u, v)}{x_{1}-x} \\
& =\frac{f\left(u_{1}, v_{1}\right)-f\left(u, v_{1}\right)}{u_{1}-u} \frac{u_{1}-u}{x_{1}-x}+\frac{f\left(u, v_{1}\right)-f(u, v)}{v_{1}-v} \frac{v_{1}-v}{x_{1}-x} .
\end{aligned}
$$

Lässt man $x_{1}$ zur Grenze $x$ und damit $u_{1}, v_{1}, y_{1}$ zu den Grenzen $u, v, y$ gehen, dann wird

$$
\begin{gathered}
\lim \frac{u_{1}-u}{x_{1}-x}=\frac{d u}{d x}, \lim \frac{v_{1}-v}{x_{1}-x}=\frac{d v}{d x}, \lim \frac{y_{1}-y}{x_{1}-x}=\frac{d y}{d x}, \\
\lim \frac{f\left(u, v_{1}\right)-f(u, v)}{v_{1}-v}=\frac{\partial f(u, v)}{\partial v}
\end{gathered}
$$

dagegen ist die Grenze des ersten Quotienten auf der rechten Seite der letzten Gleichung nicht unmittelbar ersichtlich. Nun erkennt man aber, dass

$$
\frac{f\left(u_{1}, v_{1}\right)-f\left(u, v_{1}\right)}{u_{1}-u}-\frac{f\left(u_{1}, v\right)-f(u, v)}{u_{1}-u}
$$


mit $\Delta x$ sich gleichzeitig der Null nähert. Die Grenze dieser Differenz ist also Null, und somit

$$
\lim \frac{f\left(u_{1}, v_{1}\right)-f\left(u, v_{1}\right)}{u_{1}-u}=\lim \frac{f\left(u_{1}, v\right)-f(u, v)}{u_{1}-u}=\frac{\partial f(u, v)}{\partial u} .
$$

Daher erhalten wir

$$
\frac{d y}{d x}=\frac{d f(u, v)}{d x}=\frac{\partial f(u, v)}{\partial u} \frac{d u}{d x}+\frac{\partial f(u, v)}{\partial v} \frac{d v}{d x}=\frac{\partial y}{\partial u} \frac{d u}{d x}+\frac{\partial y}{\partial v} \frac{d v}{d x} .
$$

Diesen Ausdruck nennen wir den totalen Differentialquotienten von $y$ nach $x$. Wir sehen: Sind $u$ und $v$ Funktionen von $x$, und ist $y$ eine Funktion von $u$ und $v$, so differentiiert man diese total nach der vorstehenden Formel durch Verwendung der partiellen Differentialquotienten der Funktion nach $u$ und $v$.

1. Beispiel: Sind $u$ und $v$ zu einer Summe oder Differenz verbunden, so ist $y=f(u, v)=u \pm v, \frac{\partial f}{\partial u}=1, \frac{\partial f}{\partial v}= \pm 1$ und $\frac{d y}{d x}=\frac{d u}{d x} \pm \frac{d v}{d x}$. (Vgl. Formel 7.)

2. Beispiel. Ist $y=f(u, v)=u \cdot v$; so wird $\frac{\partial f}{\partial u}=v$, $\frac{\partial f}{\partial v}=u, \frac{d y}{d x}=v \frac{d u}{d x}+u \frac{d v}{d x}$. (Vgl. Formel 8.)

3. Beispiel. Ist $y=f(u, v)=\frac{u}{v}$, so wird $\frac{\partial f}{\partial u}=\frac{1}{v}$, $\frac{\partial f}{\partial v}=-\frac{u}{v^{2}}, \frac{d y}{d x}=\frac{1}{v} \frac{d u}{d x}-\frac{u}{v^{2}} \frac{d v}{d x}=\frac{v u^{\prime}-u v^{\prime}}{v^{2}}$.

(Vgl. Formel 9.)

4. Beispiel. Wenn $y$ eine Funktion nur von $u$ ist, indem die andere Variabele $v$ ganz fehlt, dann fällt in (10) des zweite Teil der rechten Seite fort; ferner ist $\frac{\partial f}{\partial u}$ gleichbedeutend mit $\frac{d f}{d u}$ oder $\frac{d y}{d u}$. Für $y=f(u), u=\varphi(x)$ finden wir dann: $\frac{d y}{d x}=\frac{d y}{d u} \cdot \frac{d u}{d x}$. (Vgl. Formel 6.) 
Es erübrigt uns jetzt noch, zu zeigen, auf welche Weise (10) erweitert werden kann. Aus den drei Funktionen von $x$, nämlich $u=\varphi(x), v=\psi(x), w=\varrho(x)$ setzen wir die Funktion $y=f(u, v, w)$ zusammen, legen der unabhängigen Variabelen $x$ die Werte $x$ und $x_{1}$ bei, bilden die entsprechenden Funktionswerte:

$$
\begin{array}{llll}
u=\varphi(x), & v=\phi(x), & w=\varrho(x), & y=f(u, v, w) \\
u_{1}=\varphi\left(x_{1}\right), & v_{1}=\psi\left(x_{1}\right), & w_{1}=\varrho\left(x_{1}\right), & y_{1}=f\left(u_{1}, v_{1}, w_{1}\right)
\end{array}
$$

und daraus den Differenzenquotienten:

$$
\frac{y_{1}-y}{x_{1}-x}=\frac{f\left(u_{1}, v_{1}, w_{1}\right)-f(u, v, w)}{x_{1}-x},
$$

den wir, um ihn zum Übergang zur Grenze geeignet zu machen folgendermassen umgestalten:

$$
\begin{gathered}
\frac{y_{1}-y}{x_{1}-x}=\frac{f\left(u_{1}, v_{1}, w_{1}\right)-f\left(u, v_{1}, w_{1}\right)}{u_{1}-u} \frac{u_{1}-u}{x_{1}-x} \\
+\frac{f\left(u, v_{1}, w_{1}\right)-f\left(u, v, w_{1}\right)}{v_{1}-v} \frac{v_{1}-v}{x_{1}-x}+\frac{f\left(u, v, w_{1}\right)-f(u, v, w)}{w_{1}-w} \frac{w_{1}-w}{x_{1}-x} .
\end{gathered}
$$

Geht jetzt $x_{1}$ in $x$ uber, so verschwinden zugleich die Differenzen $\left(y_{1}-y\right),\left(u_{1}-u\right),\left(v_{1}-v\right),\left(w_{1}-w\right)$ und die drei aus Differenzen der Funktionswerte bestehenden Zähler, und die Betrachtungen, welche oben zur Formel (10) fuhrten, ergeben hier

$$
\frac{d y}{d x}=\frac{\partial f}{\partial u} \cdot \frac{d u}{d x}+\frac{\partial f}{\partial v} \cdot \frac{d v}{d x}+\frac{\partial f}{\partial w} \cdot \frac{d w}{d x} .
$$

Algebraische und transcendente Funktionen. Unter den algebraischen Operationen versteht man die Addition, Subtraktion, Multiplikation, Division und das Potenzieren mit rationalen, konstanten Exponenten. Wenn die unabhängige Variabele mit den konstanten Grössen nur durch eine endliche Anzahl solcher algebraischer Operationen verbunden ist, so nennt man die Funktion eine algebraische Funktion. Dabei ist es aber wohl möglich, dass eine algebraische Funktion auch durch eine unendliche Anzahl von algebraischen Operationen hervorgerufen wird. So ist ja z. B. 
$1+x+x^{2}+\ldots+x^{n}+x^{n+1}+\ldots=\lim _{n=\infty} \sum_{k=0}^{n} x^{k}=\frac{1}{1-x}$

eine algebraische Funktion. Funktionen, welohe nicht algebraisch sind, heissen transcendente Funktionen. Insbesondere gehören hierher die Exponentialfunktionen $e^{x}$ und $a^{x}$ nebst ihren Umkehrungen $\lg x$ und $\operatorname{Lg} x$; ferner die trigonometrischen Funktionen $\sin x, \cos x, \ldots$ und als deren Umkehrungen die cyklometrischen Funktionen are $\sin x$, $\arccos x, \ldots$

§3. Aufgaben zur Differentiation algebraiseher Funktionen.

1) $y=a$

2) $y=a x$

3) $y=a x+b$

4) $y=a x^{2}$

5) $y=2 x^{8}$

6) $y=4 a x^{5}$

7) $y=-\frac{5 x^{3}}{a}$

8) $y=a+2 b x+c x^{2}$

9) $y=\frac{a}{x}=a x^{-1}$

10) $y=\frac{a}{x^{4}}$

11) $y=\sqrt{x}=x^{\frac{1}{2}}$

12) $y=\sqrt[3]{x^{2}}=x^{\frac{2}{3}}$

13) $y=\sqrt[n]{x^{p}}$

14) $y=\frac{1}{\sqrt{x}}=x^{-\frac{1}{2}}$

$$
\begin{aligned}
& \frac{d y}{d x}=0 \\
& "=a \\
& "=a \\
& n=2 a x \\
& "=6 x^{2} \\
& "=20 a x^{4} \\
& n=\frac{-15 x^{2}}{a} \\
& n=2 b+2 c x \\
& n=\frac{-a}{x^{2}}
\end{aligned}
$$$$
\eta=\frac{-4 a}{x^{5}}
$$$$
\eta=\frac{1}{2 \sqrt{x}}
$$$$
n=\frac{2}{3} \cdot \frac{1}{\sqrt[3]{x}}
$$$$
"=\frac{p}{n} \cdot \sqrt[n]{x^{p-n}}
$$

$\eta=-\frac{1}{2} \cdot \frac{1}{\sqrt{x^{8}}}$ 
15) $y=\frac{1}{\sqrt[5]{x^{3}}}$

16) $y=x^{3} \sqrt{x}$

17) $y=\frac{a}{x^{3} \sqrt{x}}$

18) $y=\frac{\sqrt{x}}{x^{2} \sqrt[3]{x}}$

19) $y=x \sqrt{x \sqrt{x}}$

20) $y=\sqrt[3]{\frac{x^{3} \sqrt{x}}{m}}$

$$
\frac{d y}{d x}=-\frac{3}{20} \cdot \frac{1}{\sqrt[4]{x^{2}}}
$$$$
n=\frac{7}{2} \cdot \sqrt{x^{5}}
$$$$
n=-\frac{7}{2} \cdot \frac{a}{\sqrt{x^{0}}}
$$$$
n=-\frac{11}{6} \cdot \frac{1}{\sqrt[6]{x^{17}}}
$$$$
n=\frac{7}{4} \cdot \sqrt[4]{x^{3}}
$$$$
n=\frac{7}{6} \sqrt[6]{\frac{x}{m^{2}}} \text {. }
$$

Die nächstfolgenden Beispiele werden durch Einfthrung einer Zwischenvariabelen $\approx$ nach der unter (6) angeftuhrten Formel differentiiert:

21) $y=f(z), z=\varphi(x)$

22) $y=(a+b x)^{2}=z^{2}$

23) $y=(a-b x)^{8}$

24) $y=\left(a+b x^{2}\right)^{2}$

25) $y=\left(a-b x^{3}\right)^{5}$

26) $y=\left(a+x^{2}\right)^{3}$

27) $y=\left(a-\frac{1}{x}\right)^{3}$

28) $y=\left(a+b x+c x^{2}\right)^{n}$

29) $y=\frac{1}{a+b x}=z^{-1}$

30) $y=\frac{1}{\left(a-b x^{2}\right)^{8}}$

31) $y=\frac{1}{\left(b-x^{p}\right)^{n}}$

32) $y=\frac{2}{x^{2}}+\frac{3}{(1-x)^{3}}$ $\frac{d y}{d x}=\frac{d y}{d z} \cdot \frac{d z}{d x}$

$n=2 b(a+b x)$

$n=-3 b(a-b x)^{2}$

$\eta=4 b x\left(a+b x^{2}\right)$

$"=-15 b x^{2}\left(a-b x^{3}\right)^{4}$

$n=6 x\left(a+x^{2}\right)^{2}$

$"=\frac{3(a x-1)^{2}}{x^{4}}$

$\eta=n\left(a+b x+c x^{2}\right)^{n-1} \cdot(b+2 c x)$

$"=\frac{-b}{(a+b x)^{2}}$

$n=\frac{6 b x}{\left(a-b x^{2}\right)^{4}}$

$n=\frac{p n x^{p-1}}{\left(b-x^{p}\right)^{n+1}}$.

$n=-\frac{4}{x^{3}}+\frac{9}{(1-x)^{4}}$ 
33) $y=\sqrt{a+b x}=z^{\frac{1}{2}}$

$$
\frac{d y}{d x}=\frac{b}{2 \sqrt{a+b x}}
$$

34) $y=\sqrt{2 p x}$

$$
n=\frac{p}{\sqrt{2 p x}}
$$

35) $y=\sqrt{f(x)}$

$n=\frac{f^{\prime}(x)}{2 \sqrt{f(x)}}$

36) $y=\frac{1}{\sqrt{a-b x}}=z^{-\frac{1}{2}}$

$n=\frac{b}{2 \sqrt{(a-b x)^{3}}}$

37) $y=\sqrt{a-b x^{2}}$

$n=\frac{-b x}{\sqrt{a-b x^{2}}}$

38) $y=\sqrt{2 a x+x^{2}}$

$n=\frac{a+x}{\sqrt{2 a x+x^{2}}}$

39) $y=\sqrt{1-x^{4}}$

$"=\frac{-2 x^{3}}{\sqrt{1-x^{4}}}$

$"=\frac{1}{2} \cdot \frac{\sqrt{a-x}-\sqrt{a+x}}{\sqrt{a^{2}-x^{2}}}$

41) $y=\frac{1}{\sqrt{2 a x-x^{2}}}$

$n=\frac{x-a}{\sqrt{\left(2 a x-x^{2}\right)^{3}}}$

42) $y=\frac{1}{\sqrt[3]{\left(2-x^{3}\right)^{4}}}$

43) $y=\frac{1}{\sqrt[4]{(x-a)^{3}}}$

$n=\frac{4 x^{2}}{\sqrt[3]{\left(2-x^{3}\right)^{7}}}$

$n=\frac{-3}{4 \sqrt[4]{(x-a)^{7}}}$

44) $y=\frac{1}{\sqrt[n]{(a+b x)^{p}}}$

$n=-\frac{p}{n} \cdot \frac{b}{\sqrt[n]{(a+b x)^{p+n}}}$.

Bei den folgenden Beispielen kommen ausser der angeführten Substitutionsformel auch dio zwei folgenden zur Anwendung:

45) $y=u \cdot v$

$$
\frac{d y}{d x}=v \cdot \frac{d u}{d x}+u \cdot \frac{d v}{d x}
$$

46) $y=\frac{u}{v}$

$n=\frac{v \cdot \frac{d u}{d x}-u \cdot \frac{d v}{d x}}{v^{2}}$

47) $y=\left(a^{2}+x^{2}\right)\left(a^{2}-x^{2}\right)$

$n=-4 x^{8}$ 
48) $y=(a+b x)(a-b x)$

49) $y=(1-2 x)(1+3 x)$

50) $y=x^{4}\left(a-2 x^{3}\right)^{2}$

51) $y=(a+x) \sqrt{a-x}$

52) $y=x \sqrt{1+x}$

53) $y=x^{2} \sqrt{a-x}$

54) $y=x \sqrt{1-x^{2}}$

55) $y=\frac{a}{x} \sqrt{a^{2}+x^{2}}$

56) $y=\frac{x^{2}+2 x+1}{x^{3}-1}$

57) $y=\frac{1-x}{1+x}$

58) $y=\frac{1-2 x^{2}}{2-x^{2}}$

59) $y=\frac{x^{2}-2 x+3}{x^{2}+2 x-3}$

60) $y=\frac{\left(a+x^{2}\right)^{3}}{\left(b-x^{8}\right)^{2}}$

61) $y=\frac{1+\sqrt{x}}{1-\sqrt{x}}$

62) $y=\frac{\sqrt{a+b x}}{x}$

63) $y=\frac{x}{\sqrt{1+x}}$

64) $y=\sqrt{\frac{a+b x}{a-b x}}$

65) $y=\frac{1}{x \sqrt{a+x}}$ $\frac{d y}{d x}=-2 b^{2} x$

$n=1-12 x$

$n=4 x^{3}\left(a-2 x^{3}\right)\left(a-5 x^{3}\right)$

$n=\frac{a-3 x}{2 \sqrt{a-x}}$

$n=\frac{2+3 x}{2 \sqrt{1+x}}$

$n=\frac{4 a x-5 x^{2}}{2 \sqrt{a-x}}$

$n=\frac{1-2 x^{2}}{\sqrt{1-x^{2}}}$

$n=\frac{-a^{8}}{x^{2} \sqrt{a^{2}+x^{2}}}$

$"=-\frac{x^{4}+4 x^{3}+3 x^{2}+2 x+2}{\left(x^{3}-1\right)^{2}}$

$n=\frac{-2}{(1+x)^{2}}$

$n=\frac{-6 x}{\left(2-x^{2}\right)^{2}}$

$\eta=\frac{4 x(x-3)}{\left(x^{2}+2 x-3\right)^{2}}$

$n=\frac{6 x\left(a+x^{2}\right)^{2}(b+a x)}{\left(b-x^{3}\right)^{3}}$

$"=\frac{1}{\sqrt{x}(1-\sqrt{x})^{2}}$

$n=-\frac{2 a+b x}{2 x^{2} \sqrt{a+b x}}$

$=\frac{2+x}{2 \sqrt{(1+x)^{8}}}$

$n=\frac{a b}{(a-b x)^{2}} \sqrt{\frac{a-b x}{a+b x}}$

.$=-\frac{2 a+3 x}{2 x^{2} \sqrt{(a+x)^{3}}}$ 
66)

$y=\sqrt{\frac{x}{x^{2}-a^{2}}}$

67) $y=(x+\sqrt{1+x})^{2}$

$$
\begin{aligned}
\frac{d y}{d x} & =-\frac{x^{2}+a^{2}}{2 \sqrt{x\left(x^{2}-a^{2}\right)^{3}}} \\
n & =\frac{2+3 x+(2 x+1) \sqrt{1+x}}{\sqrt{1+x}} .
\end{aligned}
$$

§4. Die Differentialquotienten der trigonometrisehen und eyklometrisehen Funktionen.

Um die Funktion $y=\sin x$ differentiieren zu können, erinnern wir an zwei einfache geometrische Sätze:

Fig. 2.

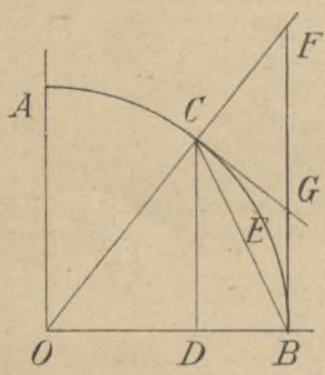

Fig. 3.

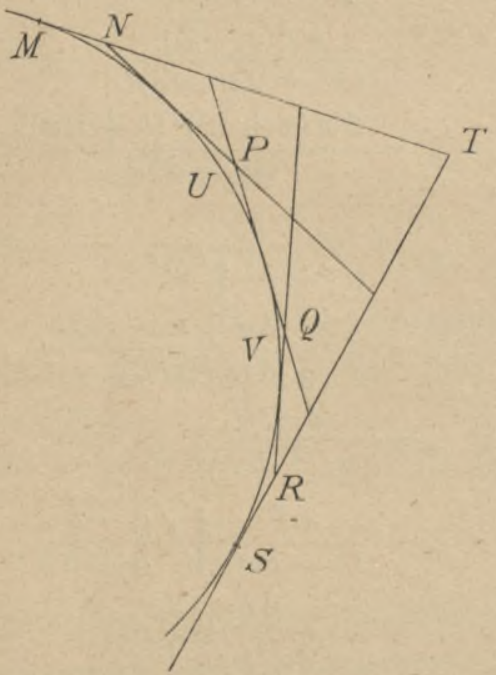
so wird

Ist $A B O$ in Fig. 2 ein Kreisquadrant, so wird $C D<C B<C E B$ für jeden Punkt $C$ zwischen $A$ und $B$, dagegen $C D=C B=C E B$, wenn $C$ mit $B$ zusammenfällt. Daher ist $\sin \alpha \leqq \alpha$ für kleine Winkel $C O B=\alpha$.

Ist $M U V S$ in Fig. 3 ein beliebiger stets konvexer Bogen und sind $M N$, $N P, P Q, Q R, R S$ Tangenten an ihm,

$$
\begin{gathered}
M N+N P+P Q+Q R \\
+R S<M T+T S,
\end{gathered}
$$

und zwar bleibt diese Beziehung richtig, wie viele Zwischentangenten auch zwischen $M T$ und $S T$ gezogen sein mögen. Lässt man die Anzahl der Zwischentangenten ins Unendliche wachsen und die Beruhrungspunk te aufeinander folgender Tangenten näher und näher an einander rücken, so folgt, dass $M U V S<M T+T S$ wird. Fällt $M$ mit $S$ zusammen, so wird $M U V S=M T+T S$. 
Bedeuten daher $C G$ und $G B$ in Fig. 2 Tangenten in $C$ und $B$, so ist $C E B<C G+G B<F G+G B=F B$, d. h. $\alpha \leqq \operatorname{tang} \alpha$, wobei sich das Gleichzeichen auf den Winkel $\alpha=0$ bezieht.

Man hat daher

$$
\begin{gathered}
\sin \alpha \leqq \alpha, \alpha \cos \alpha \leqq \sin \alpha \\
\alpha \cos \alpha \leqq \sin \alpha \leqq \alpha \\
\cos \alpha \leqq \frac{\sin \alpha}{\alpha} \leqq 1
\end{gathered}
$$

Lässt man $\alpha$ zur Grenze 0 gehen, so wird in diesem Ausdrucke die linke Seite $=1$, und demnach:

$$
\lim _{\alpha=0} \frac{\sin \alpha}{\alpha}=1 \text {. }
$$

Nach diesen Vorbereitungen setzen wir:

$$
\begin{aligned}
y & =\sin x, \quad y_{1}=\sin x_{1}, \\
\frac{y_{1}-y}{x_{1}-x} & =\frac{\sin x_{1}-\sin x}{x_{1}-x}=\frac{2 \cos \frac{1}{2}\left(x_{1}+x\right) \sin \frac{1}{2}\left(x_{1}-x\right)}{x_{1}-x} \\
& =\cos \frac{1}{2}\left(x_{1}+x\right) \cdot \frac{\sin \frac{1}{2}\left(x_{1}-x\right)}{\frac{1}{2}\left(x_{1}-x\right)} .
\end{aligned}
$$

Geht man zur Grenze $x_{1}=x$ uber, so wird der letzte Bruch nach dem soeben Dargelegten gleich 1 werden. Man bekommt deshalb:

$$
\frac{d y}{d x}=\frac{d \sin x}{d x}=\cos x .
$$

Der Differentialquotient $v$ on $\sin x$ ist $\cos x$.

Auf die nämliche Weise wird auch der Differentialquotient des Cosinus hergeleitet.

$$
\begin{gathered}
y=\cos x, \quad y_{1}=\cos x_{1}, \\
\frac{y_{1}-y}{x_{1}-x}=\frac{\cos x_{1}-\cos x}{x_{1}-x}=\frac{-2 \sin \frac{1}{2}\left(x_{1}+x\right) \sin \frac{1}{2}\left(x_{1}-x\right)}{x_{1}-x} \\
=-\sin \frac{1}{2}\left(x_{1}+x\right) \frac{\sin \frac{1}{2}\left(x_{1}-x\right)}{\frac{1}{2}\left(x_{1}-x\right)} \\
\frac{d y}{d x}=\frac{d \cos x}{d x}=-\sin x
\end{gathered}
$$

Der Differentialquotient von $\cos x$ ist $-\sin x$. 
Übrigens kann diese Formel auch aus der vorhergehenden abgeleitet werden, da $y=\cos x=\sin \left(\frac{\pi}{2}-x\right)=\sin z$ ist, wenn $z=\frac{\pi}{2}-x$ gesetzt wird. Da nun $\frac{d y}{d z}=\cos z, \frac{d z}{d x}=-1$ ist, so wird $\frac{d y}{d x}=-\cos z=-\sin x$.

Die Differentialquotienten von tang $x$ und $\operatorname{cotg} x$ ergeben sich aus den vorhergehenden auf die einfachste Weise:

$y=\operatorname{tg} x=\frac{\sin x}{\cos x} ; \quad \frac{d y}{d x}=\frac{\cos ^{2} x+\sin ^{2} x}{\cos ^{2} x}=\frac{1}{\cos ^{2} x}$.

$y=\operatorname{ctg} x=\frac{\cos x}{\sin x} ; \quad \frac{d y}{d x}=\frac{-\sin ^{2} x-\cos ^{2} x}{\sin ^{2} x}=-\frac{1}{\sin ^{2} x}$.

Aufgaben:

68) $y=\sin (a x)=\sin z$

$$
\begin{aligned}
\frac{d y}{d x} & =a \cos (a x) \\
n & =-a \sin (a x) \\
" & =\frac{1}{a} \cdot \cos \frac{x}{a} \\
" & =-\sin \frac{x}{a} \\
n & =n x^{n-1} \cos \left(x^{n}\right) \\
" & =-\frac{a \sin \sqrt{a x}}{2 \sqrt{a x}} \\
n & =-\frac{a^{2}}{x^{2}} \cdot \cos \frac{a}{x} \\
n & =\frac{a}{x^{2}} \cdot \sin \frac{1}{x}
\end{aligned}
$$

69) $y=\cos (a x)$

70) $y=\sin \frac{x}{a}$

71) $y=a \cos \frac{x}{a}$

72) $y=\sin \left(x^{n}\right)=\sin z$

73) $y=\cos \sqrt{a x}$

74) $y=a \sin \frac{a}{x}$

75) $y=a \cos \frac{1}{x}$

76) $y=\sin / \frac{1}{x}$

$n=\frac{-1}{2 \sqrt{x^{3}}} \cdot \cos \sqrt{\frac{1}{x}}$

77) $y=\operatorname{tg}(a x)$

$"=\frac{a}{\cos ^{2}(a x)}$ 
78) $y=a \operatorname{tg} \frac{x}{a}$

$$
\frac{d y}{d x}=\frac{1}{\cos ^{2} \frac{x}{a}}
$$

79) $y=a \operatorname{tg} \frac{a}{x}$

$$
n=-\frac{a^{2}}{x^{2}} \cdot \frac{1}{\cos ^{2} \frac{a}{x}}
$$

80) $y=\sin (a+b x)=\sin z$

$$
"=b \cos (a+b x)
$$

81) $y=\cos \left(a-b x^{2}\right)$

$n=2 b x \sin \left(a-b x^{2}\right)$

82) $y=\sin ^{2} x=z^{2}$

$n=\sin (2 x)$

83) $y=\cos ^{2}(a x)$

$"=-a \sin (2 a x)$

84) $y=2 \sin ^{n}(a x)$

$n=n a \sin ^{n-2}(a x) \sin (2 a x)$

85) $y=\cos ^{n} \frac{a}{x}$

$n=\frac{n a}{x^{2}} \cdot \cos ^{n-1} \frac{a}{x} \sin \frac{a}{x}$

86) $y=\frac{1}{\sin x}=z^{-1}$

$"=-\frac{\operatorname{cotg} x}{\sin x}$

87) $y=\left(\frac{1}{\cos x}\right)^{n}$

$"=\frac{n \sin x}{\cos ^{n+1} x}$

88) $y=\frac{1}{2} \sin x+\sin \frac{x}{2}$

$n=\cos \frac{3 x}{4} \cos \frac{x}{4}$

89) $y=\frac{1}{12} \cos 3 x-\frac{1}{4} \cos x$

$n=-\frac{1}{2} \cdot \cos 2 x \sin x$

90) $y=\operatorname{tg} x-\operatorname{cotg} x$

$\eta=\frac{4}{\sin ^{2}(2 x)}$

91) $y=3 \sin x-4 \sin ^{3} x$

$\eta=3 \cos 3 x$

92) $y=\frac{1}{2 \sin ^{2} x}-\frac{1}{4 \sin ^{4} x}$

$"=\frac{\cos ^{3} x}{\sin ^{5} x}$

93) $y=\frac{1}{\cos x}+\operatorname{tg} x$

$\eta=\frac{2 \sin ^{2}\left(\frac{\pi}{4}+\frac{x}{2}\right)}{\cos ^{2} x}$

94) $y=\sin x \cos x$

$\eta=\cos 2 x$

95) $y=(1-\cos 2 x)^{2}$

$n=16 \sin ^{3} x \cos x$

?96) $y=2 \cos ^{2}(x+a)-\cos 2 x$

$"=-4 \cos (a+2 x) \sin a$

97) $y=(x \operatorname{tg} x)^{2}$

$n=x \operatorname{tg} x\left(\frac{\sin 2 x+2 x}{\cos ^{2} x}\right)$

98) $y=3 \sin x \cos ^{2} x+\sin ^{3} x \quad \pi=3 \cos x \cos 2 x$ 
2. 99) $y=\left(4 \sin x-8 \sin ^{8} x\right) \cos x \frac{d y}{d x}=4 \cos 4 x$

100) $y=(a+b \sin x) \cos x$

101) $y=\sin x \sin (a-x)$

$"=b \cos 2 x-a \sin x$

${ }_{n}=\sin (a-2 x)$

102) $y=\frac{a-b \cos x}{a+b \cos x}$

$n=\frac{2 a b \sin x}{(a+b \cos x)^{2}}$

203) $y=\frac{\sin x+\cos x}{\sin x-\cos x}$

$"=\frac{-1}{\cos ^{2}\left(45^{0}+x\right)}$

104) $y=\frac{\sin x+\cos x}{\sin x \cos x}$

$"=\frac{\sin ^{3} x-\cos ^{3} x}{\sin ^{2} x \cos ^{2} x}$

105) $y=\sin x-x \cos x$

$\eta=x \sin x$.

Die cyklometrischen Funktionen sind die Umkehrungen der trigonometrischen; deshalb lassen sich auch ihre Differentialquotienten aus denjenigen der trigonometrischen Funktionen durch Umkehrung ableiten.

1. $y=\arcsin x$

ist die Umkehrung von $x=\sin y$. Nun ist:

$$
\frac{d x}{d y}=\cos y=\sqrt{1-\sin ^{2} y}=\sqrt{1-x^{2}}
$$

und daraus entsteht:

$$
\frac{d y}{d x}=\frac{1}{\sqrt{1-x^{2}}} .
$$

$$
\text { 2. } y=\arccos x
$$

gehört zu $x=\cos y$. Da nun:

$$
\frac{d x}{d y}=-\sin y=-\sqrt{1-\cos ^{2} y}=-\sqrt{1-x^{2}}
$$

ist, so folgt: $\quad \frac{d y}{d x}=-\frac{1}{\sqrt{1-x^{2}}}$.

Kturzer ist die folgende Ableitung:

$$
y=\arccos x=\frac{\pi}{2}-\arcsin x ; \quad \frac{d y}{d x}=-\frac{1}{\sqrt{1-x^{2}}}
$$

3. $y=\operatorname{arctg} x$

Es ist $x=\operatorname{tg} y, \frac{d x}{d y}=\frac{1}{\cos ^{2} y}=1+\operatorname{tg}^{2} y=1+x^{2}$, und daraus 
durch Umkehrung:

$$
\frac{d y}{d x}=\frac{1}{1+x^{2}} .
$$

$$
\text { 4. } y=\operatorname{arccotg} x \text {. }
$$

Aus $y=\frac{\pi}{2}-\operatorname{arctg} x$ erhält man leicht:

$$
\frac{d y}{d x}=-\frac{1}{1+x^{2}} \text {. }
$$

Die Ableitung wäre auch so möglich:

$$
\begin{gathered}
y=\operatorname{arccotg} x=\operatorname{arctg} \frac{1}{x}=\operatorname{arctg} z, \text { wenn } z=\frac{1}{x} . \\
\text { Nun ist } \frac{d y}{d z}=\frac{1}{1+z^{2}} ; \quad \frac{d z}{d x}=-\frac{1}{x^{2}} \text { und } \\
\frac{d y}{d x}=\frac{1}{1+z^{2}} \cdot \frac{-1}{x^{2}}=-\frac{1}{1+x^{2}} .
\end{gathered}
$$

Aufgaben.

106) $y=\arcsin (a x)$

107) $y=\arccos (a-x)$

108) $y=\arcsin \frac{a^{2}-x^{2}}{a^{2}+x^{2}}$

109) $y=\arcsin \frac{1}{x}$

110) $y=\arcsin \sqrt{1-x^{2}}$

111) $y=\arccos \frac{b+a \cos x}{a+b \cos x}$

112) $y=\arccos \left(\frac{a-x}{x}\right)$

113) $y=\frac{1}{\sqrt{\bar{b}}} \cdot \arcsin x / \frac{\bar{b}}{\bar{a}}$

$$
\frac{d y}{d x}=\frac{a}{\sqrt{1-a^{2} x^{2}}}
$$

$$
n=\frac{1}{\sqrt{1-(a-x)^{2}}}
$$$$
\eta=\frac{-2 a}{a^{2}+x^{2}}
$$$$
n=-\frac{1}{x \sqrt{x^{2}-1}}
$$$$
n=\frac{-1}{\sqrt{1-x^{2}}}
$$$$
"=\frac{\sqrt{a^{2}-b^{2}}}{a+b \cos x}
$$$$
n=\frac{a}{x \sqrt{2 a x-a^{2}}}
$$$$
=\frac{1}{\sqrt{a-b x^{2}}}
$$ 
2.114) $y=\arcsin \frac{x}{\sqrt{1+x^{2}}} \quad \frac{d y}{d x}=\frac{1}{1+x^{2}}$

115) $y=\operatorname{arctg} \sqrt{a x}$

116) $y=\operatorname{arctg} \frac{x}{a-x}$

117) $y=\operatorname{arctg} \frac{1}{x-2}$

118) $y=\operatorname{arctg} \sqrt{\frac{1-x}{1+x}}$

119) $y=\operatorname{arccotg} \sqrt{2-x}$

$"=\frac{a}{2(1+a x) \sqrt{a x}}$

$n=\frac{a}{(a-x)^{2}+x^{2}}$

$n=\frac{-1}{1+(x-2)^{2}}$

n $=\frac{-1}{2 \sqrt{1-x^{2}}}$

$n=\frac{1}{2(3-x) \sqrt{2-x}}$

120) $y=\operatorname{arccotg} \frac{x}{\sqrt{1-x^{2}}}$

$"=\frac{-1}{\sqrt{1-x^{2}}}$

121) $y=\operatorname{arccotg} \frac{\sqrt{1+x^{2}}-1}{x}$

$$
\begin{aligned}
& n=\frac{-1}{2\left(1+x^{2}\right)} \\
& n=-1 \\
& n=\frac{m}{m^{2}+\left(1-m^{2}\right) \cos ^{2} x} \\
& n=\frac{1}{2(x+1) \sqrt{x}}
\end{aligned}
$$

124) $y=\operatorname{arccotg} \frac{1}{\sqrt{x}}$

? 125) $y=\operatorname{arc}\left(\operatorname{tg}=a \operatorname{tg} \frac{x}{2}\right)$

$$
n=\frac{\frac{1}{2} a}{1+\left(a^{2}-1\right) \sin ^{2} \frac{x}{2}}
$$

?126) $y=a \cdot \arcsin \frac{\sqrt{2 a x-x^{2}}}{a}$

$-\sqrt{2 a x-x^{2}}$

$$
\frac{d y}{d x}=\frac{x}{\sqrt{2 a x-x^{2}}}
$$

127) $y=\sqrt{a x-x^{2}}-a \cdot \operatorname{arctg} \sqrt{\frac{a-x}{x}}$

$$
\frac{d y}{d x}=\sqrt{\frac{a-x}{x}}
$$

128) $y=\left(x-\frac{1}{2} \operatorname{arctg} x\right) \cdot \operatorname{arctg} x, y=\frac{x+x^{2} \operatorname{arctg} x}{1+x^{2}}$. 
§ 5. Exponential- und logarithmisehe Funktionen.

In der für positive ganze $n$ gültigen Binomialentwickelung: $\left(1+\frac{x}{n}\right)^{n}=1+\frac{x}{1}+\frac{n}{1} \frac{n-1}{2} \frac{x^{2}}{n^{2}}+\frac{n}{1} \frac{n-1}{2} \frac{n-2}{3} \frac{x^{3}}{n^{3}}+\ldots$ schreiben wir die rechte Seite:

$$
\begin{gathered}
1+\frac{x}{1}+\left(1-\frac{1}{n}\right) \frac{x^{2}}{1.2}+\left(1-\frac{1}{n}\right)\left(1-\frac{2}{n}\right) \frac{x^{8}}{12.3}+\cdots \\
\ldots+\left(1-\frac{1}{n}\right)\left(1-\frac{2}{n}\right) \cdots\left(1-\frac{n-1}{n}\right) \frac{x^{n}}{1.2 .3 \ldots n} .
\end{gathered}
$$

Wir haben die Absicht, $n$ ins Unendliche wachsen zu lassen. Da aber der Satz, dass die Grenze einer Summe gleich der Summe der Grenzen der Summanden ist (S. 6, letzte Zeile), nur für eine endliche Gliederzahl gilt, während hier mit wachsendem $n$ die Anzahl der Glieder ins Unendliche wächst, so können wir den Übergang nicht direkt vornehmen. Es sei $p$ eine positive ganze Zahl $<n$, und der absolute Betrag $|x|$ kleiner als $p$. Wir brechen die Reihe hinter dem Gliede mit $x^{p}$ ab und bezeichnen den Rest mit $R_{p, n}$. Also

$$
\left(1+\frac{x}{n}\right)^{n}
$$

$=1+\frac{x}{1}+\left(1-\frac{1}{n}\right) \frac{x^{2}}{1 \cdot 2}+\left(1-\frac{1}{n}\right)\left(1-\frac{2}{n}\right) \frac{x^{3}}{1.2 .3}+\cdots$

$+\left(1-\frac{1}{n}\right)\left(1-\frac{2}{n}\right) \cdots\left(1-\frac{p-1}{n}\right) \frac{x^{p}}{1.2 .3 \ldots p}+R_{p, n}$.

Der Rest $R_{p, n}$ liegt zwischen der Summe aller folgenden negativ genommenen und aller folgenden positiv genommenen Glieder. Fur seinen absoluten Wert gilt demnach

$$
\begin{aligned}
& \left|R_{p, n}\right|<\frac{|x|^{p+1}}{1.2 \ldots(p+1)}+\frac{|x|^{p+2}}{1.2 \ldots(p+2)}+\ldots+\frac{|x|^{n}}{1.2 \ldots n} \\
& <\frac{|x|^{p+1}}{1.2 \ldots(p+1)}\left[1+\left(\frac{|x|}{p+1}\right)+\left(\frac{|x|}{p+1}\right)^{2}+\ldots\right]
\end{aligned}
$$

Die Summe in der letzten Klammer kann beliebig weit fortgesetzt werden. Da $|x|<p$ ist, so folgt hieraus duroh Summation der Reihe als Wert des letzten Ausdruckes 


$$
\frac{|x|^{p+1}}{1.2 \ldots(p+1)} \frac{1}{1-\frac{|x|}{p+1}} .
$$

Wir haben demnach

$$
0<\left|R_{p, n}\right|<\frac{|x|^{p+1}}{1.2 .3 \ldots(p+1)} \cdot \frac{1}{1-\frac{|x|}{p+1}} .
$$

Aus dieser Form erkennt man, dass $(B)$ zwei für jedes $n>p$ gultige Grenzen von $\left|R_{p, n}\right|$ giebt. Denn die linke wie die rechte Seite der doppelten Ungleichung ist von $n$ unabhängig. Insbesondere gilt $(B)$ auch noch für beliebig grosse $n$. Lässt man also die Zahl $n$ in $(A)$ uber alle Grenzen hinaus wachsen, und bezeichnet den Grenzwert $\lim _{n=\infty} R_{p, n}=R_{p}$, so kann man jetzt bei einer Summe von $p$ Gliedern die Grenze der Summe durch die Summe der Grenzen der Summanden ersetzen und bekommt

$$
\begin{aligned}
\lim _{n=\infty}\left(1+\frac{x}{n}\right)^{n} & =1+\frac{x}{1}+\frac{x^{2}}{1.2}+\frac{x^{3}}{1.2 .3}+\ldots+\frac{x^{p}}{1.2 \ldots p}+R_{p} \\
0 & <\left|R_{p}\right|<\frac{|x| p+1}{1.2 \ldots(p+1)} \frac{1}{1-\frac{|x|}{p+1}} .
\end{aligned}
$$

In (C) wollen wir nun auch, nachdem die rechte Seite von $n$ frei gemacht ist, den Wert von $p$ tuber alle Grenzen waohsen lassen. Hierbei geht für jedes endliche $x$ der letzte Bruch in (D) in den Wert 1 über, und den zurückbleibenden Teil der rechten Seite von (D) können wir, wenn $k \leqq|x|<k+1$ ist, in der Form

$$
\frac{|x|^{k}}{1.2 \ldots k}\left(\frac{|x|}{k+1}\right)\left(\frac{|x|}{k+2}\right) \cdots\left(\frac{|x|}{p+1}\right)<\frac{|x|^{k}}{1.2 \ldots k}\left(\frac{|x|}{k+1}\right)^{p-k+1}
$$

schreiben. Der erste Faktor rechts ist endlich; der zweite wird sich mit wachsendem $p$ als Potenz eines echten Bruches dem Grenzwerte 0 nähern. Folglich ist $\lim R_{p}=0$, und wir haben die Entwickelung 


$$
\lim _{n=\infty}\left(1+\frac{x}{n}\right)^{n}=1+\frac{x}{1}+\frac{x^{2}}{1.2}+\frac{x^{3}}{1.2 .3}+\cdots
$$

für jedes reelle, positive oder negative $x$ in Form einer konvergenten unendlichen Reihe.

Den Zahlenwert dieser Reihe für $x=1$ bezeichnen wir mit $e$ und setzen

$$
e=\lim _{n=\infty}\left(1+\frac{1}{n}\right)^{n}=1+1+\frac{1}{1.2}+\frac{1}{1 \cdot 2 \cdot 3}+\cdots
$$

Weil nun die rechte Seite $>2$ und

$$
<1+1+\frac{1}{2}+\frac{1}{2^{2}}+\frac{1}{2^{3}}+\ldots<3
$$

ist, so muss der Wert von $e$ zwischen 2 und 3 liegen. Wir finden

$$
e=2,718281828459 \ldots
$$

In (E) ist $n$ als ganze Zahl zu denken. Bedeutet $x$ eine ganze, positive Zahl, dann kann man in dieser Formel $n$ duroh $n x$ ersetzen, und hat:

$$
\begin{aligned}
\lim \left(1+\frac{x}{n}\right)^{n} & =\lim \left(1+\frac{x}{n x}\right)^{n x}=\lim \left(1+\frac{1}{n}\right)^{n x} \\
& =\left[\lim \left(1+\frac{1}{n}\right)^{n}\right]^{x}=e^{x},
\end{aligned}
$$

wo der Übergang zur letzten Grenze möglich ist, da die Grenze eines Produkts der endlichen Faktorenzahl $x$ gleich dem Produkte der Grenzen ist. Für ganze, positive $x$ gilt sonach

$$
e^{x}=\lim _{n=\infty}\left(1+\frac{x}{n}\right)^{n}=1+\frac{x}{1}+\frac{x^{2}}{1.2}+\frac{x^{3}}{1.2 .3}+\cdots
$$

Ist ferner $y$ eine ganze, positive Zahl, so kann man $n$ hierin durch $n y$ ersetzen, und hat:

$$
\begin{gathered}
e^{x}=\lim \left(1+\frac{x}{n}\right)^{n}=\lim _{\left(1+\frac{x}{n y}\right)^{n y}=\left[\lim \left(1+\frac{\frac{x}{y}}{n}\right)^{n}\right]^{y},} \\
e^{\frac{x}{y}}=\lim _{n=\infty}\left(1+\frac{\frac{x}{y}}{n}\right)^{n}
\end{gathered}
$$

$(F)$ gilt daher für jedes rationale, positive $x$. 
Schliesst man endlich die positive, irrationale Zahl $x$ zwischen zwei rationale Brüche ein

so wird

$$
\frac{p}{q}<x<\frac{s}{t}
$$

$1+\frac{\frac{p}{q}}{1}+\frac{\left(\frac{p}{q}\right)^{2}}{1.2}+\cdots<1+\frac{x}{1}+\frac{x^{2}}{1.2}+\cdots<1+\frac{\frac{s}{t}}{1}+\frac{\left(\frac{s}{t}\right)^{2}}{1.2}+\cdots$

und daraus folgt, wenn man die Brïche von beiden Seiten her der Grösse $x$ immer näher kommen lässt, die Gültigkeit von (F) für jedes positive $x$.

In derselben Art kann man für positive oder negative $x$

$$
\lim _{n=\infty}\left(1+\frac{x}{n^{2}}\right)^{n}
$$

behandeln. Man ubersieht sofort, dass, wenn in $(B) \frac{x}{n}$ an die Stelle von $n$ tritt, der Rest $\left|R_{p, n}\right|$ für jedes $p$ bei wachsendem $n$ zu Null wird, so dass beim Grenzuibergange von $(A)$ zu $(C)$ nur das erste Glied 1 tibrig bleibt. Es wird daher für alle positiven und negativen $x$ :

$$
\lim _{n=\infty}\left(1+\frac{x}{n^{2}}\right)^{n}=1
$$

Daraus folgt, wenn man für $x$ einsetzt $-x^{2}$ :

d. b.

$$
\lim _{n=\infty}\left(1+\frac{x}{n}\right)^{n} \cdot \lim _{n=\infty}\left(1-\frac{x}{n}\right)^{n}=\lim _{n=\infty}\left(1-\frac{x^{2}}{n^{2}}\right)^{n}=1
$$

oder

$$
e^{x} \lim _{n=\infty}\left(1-\frac{x}{n}\right)^{n}=1
$$

$$
\lim _{n=\infty}\left(1+\frac{-x}{n}\right)^{n}=e^{-x}
$$

Die Formel (F) gilt daher für alle reellen Zahlen $x$ : Soll jetzt die Funktion $y=e^{x}$. differentiiert werden, so gehen wir von 


$$
\begin{aligned}
y+\Delta y & =e^{x+\Delta x}, \quad y=e^{x} ; \quad \Delta y=e^{x}\left(e^{\Delta x}-1\right) \\
\frac{\Delta y}{\Delta x} & =e^{x}\left(\frac{e^{\Delta x}-1}{\Delta x}\right)
\end{aligned}
$$

aus. Setzt $\operatorname{man} e^{\Delta x}-1=\frac{1}{n}$ d. h. $e^{\Delta x}=1+\frac{1}{n}, \Delta x=\lg \left(1+\frac{1}{n}\right)$, so wird:

$$
\frac{\Delta y}{\Delta x}=e^{x} \frac{e^{\Delta x}-1}{\Delta x}=\frac{e^{x}}{n \lg \left(1+\frac{1}{n}\right)}=\frac{e^{x}}{\lg \left(1+\frac{1}{n}\right)^{n}} .
$$

Lässt man nun $\Delta x$ zur Grenze 0 gehen, so wird $n$ über alle Grenzen wachsen, und $\lg \left(1+\frac{1}{n}\right)^{n}$ wird zu $\lg e=1$ werden. Man hat daher

$$
\frac{d y}{d x}=\frac{d e^{x}}{d x}=e^{x} .
$$

Wenn man auf der rechten Seite von (F) jedes einzelne Glied der unendlichen Reihe differentiiert, so kommt dieselbe konvergente Reihe wieder heraus. Es wäre also in diesem Falle erlaubt gewesen, die Differentiation der unendlichen Reihe gliedweise vorzunehmen.

Die Logarithmen für die Basis $e$, welche wir durch $\mathrm{lg}$ bezeichnen ( $\mathrm{vgl}$. S. 18) sollen aus Gründen, die erst später dargelegt werden köunen, die natürlichen Logarithmen heissen (vgl. Aufgabe 424).

Um die allgemeinere Exponentialfunktion $a^{x}$ zu differentiieren, setzen wir $a=e^{m}$ und haben alsdann $m=\lg a, \mathrm{~d}$. h. es ist $m$ der natürliche Logarithmus von a. Da nun $y=e^{m x}=e^{z}$, wenn $m x=z$ gesetzt wird, so findet man

$$
\frac{d y}{d z}=e^{z}, \quad \frac{d z}{d x}=m \text { und } \frac{d y}{d x}=m e^{z}=m e^{m x}=a^{x} \lg a .
$$

Die Differentialquotienten der beiden logarithmischen Funktionen gehen wieder durch Umkehrung aus den vorhergehenden hervor. Ist:

$$
y=\lg x,
$$

Dölp, Aufgaben. 


$$
\text { so ist } x=c^{y}, \frac{d x}{d y}=e^{y}, \frac{d y}{d x}=\frac{1}{e^{y}}=\frac{1}{x} .
$$

Ebenso gehört zu der Funktion:

$$
y=\operatorname{Lg} x
$$

die Umkehrung $x=a^{y}$, und $\frac{d x}{d y}=a^{y} \lg a$, woraus dann:

$$
\frac{d y}{d x}=\frac{1}{a^{y} \cdot \lg a}=\frac{1}{\lg a} \cdot \frac{1}{x} .
$$

\section{Aufgaben.}

129) $y=e^{a x}=e^{z}$

130) $y=e^{-a x}$

131) $y=a e^{\frac{x}{a}}$

132) $y=e^{x x}$

133) $y=e^{2 \vee} \overline{a x}$

134) $y=e^{\cos x}$

135) $y=e^{\sin ^{2} x}$

136) $y=e^{\operatorname{arctg} x}$

137) $y=x^{2} e^{x}$

138) $y=e^{x}(x-1)$

139) $y=e^{x} x^{n}$

140) $y=\left(x^{2}-2 x+2\right) e^{x}$

141) $y=a\left(e^{\frac{x}{a}}+e^{\left.-\frac{x}{a}\right)^{2}}\right.$

142) $y=\cos x e^{\sin x}$

143) $y=e^{2 x} \sin ^{2} x$

144) $y=\frac{e^{x}}{x^{n}}$

145) $y=\frac{e^{x}-1}{e^{x}+1}$

146) $y=\frac{e^{x}(x-n)}{x^{n}}$

$$
\frac{d y}{d x}=a e^{a x}
$$

$$
\begin{aligned}
& \eta=-a e^{-a x} \\
& \eta=e^{\frac{x}{a}} \\
& "=2 x e^{x x} \\
& n=\frac{a e^{2} \sqrt{a x}}{\sqrt{a x}}
\end{aligned}
$$$$
"=-\sin x e^{\cos x}
$$$$
n=\sin 2 x e^{\sin ^{2} x}
$$$$
"=\frac{e^{\operatorname{arctg} x}}{1+x^{2}}
$$$$
\eta=x e^{x}(2+x)
$$$$
n=x e^{x}
$$$$
n=(x+n) e^{x} x^{n-1}
$$$$
n=x^{2} e^{x}
$$$$
n=2\left(e^{\frac{2 x}{a}}-e^{\left.-\frac{2 x}{a}\right)}\right.
$$$$
n=\left(\cos ^{2} x-\sin x\right) e^{\sin x}
$$$$
n=e^{2 x}\left(2 \sin ^{2} x+\sin 2 x\right)
$$$$
n=\frac{e^{x}(x-n)}{x^{n+1}}
$$$$
n=\frac{2 e^{x}}{\left(e^{x}+1\right)^{2}}
$$

$n=\frac{e^{x}\left[x+(x-n)^{2}\right]}{x^{n+1}}$ 
147) $y=\sqrt{e^{a x}}$

148) $y=\sqrt{x\left(e^{x}+1\right)}$

149) $y=a \sqrt{x x+1}$

150) $y=a^{\operatorname{tg} x}$

151) $y=\left(a^{m x}+b\right)^{p}$

152) $y=a^{x} \cdot x^{a}$

153) $y=\lg (a-x)$

154) $y=\lg \left(x^{n}\right)=n \cdot \lg x$

155) $y=\lg \frac{1}{x}=-\lg x$

156) $y=\lg (a x)=\lg +\lg x \quad n=\frac{1}{x}$

157) $y=(\lg x)^{n}=z^{n}$

158) $y=\lg \left(\frac{a}{a+x}\right)$

159) $y=\lg x+\lg x^{2}=3 \lg x \quad$, $=\frac{3}{x}$

160). $y=\lg \left(1-x^{2}\right)=\lg z$

161) $y=\lg \left(1+\frac{a}{x}\right)$

162) $y=\lg (a-\sqrt{x})$

163) $y=\lg \left(e^{m x}+e^{-m x}\right)$

164) $y=\lg \left(x+\sqrt{1+x^{2}}\right)$ $\frac{d y}{d x}=\frac{a}{2} \sqrt{e^{a x}}$

$n=\frac{e^{x}(x+1)+1}{2 \sqrt{x\left(e^{x}+1\right)}}$

$n=\frac{x a \sqrt{x x+1} \lg a}{\sqrt{x^{2}+1}}$

$n=\frac{a^{\operatorname{tg} x} \lg a}{\cos ^{2} x}$

$n=p\left(a^{m x}+b\right)^{p-1} a^{m x} m \lg a$

$n=a^{x} x^{a-1}(a+x \lg a)$

$n=-\frac{1}{a-x}$

$n=\frac{n}{x}$

$n=-\frac{1}{x}$

$n=\frac{n(\lg x)^{n-1}}{x}$

$n=-\frac{1}{a+x}$

$\eta=-\frac{2 x}{1-x^{2}}$

$n=-\frac{a}{x(a+x)}$

$n=\frac{1}{2(x-a \sqrt{x})}$

$n=\frac{m\left(e^{m x}-e^{-m x}\right)}{e^{m x}+e^{-m x}}$

$=\frac{1}{\sqrt{1+x^{2}}}$ 
165) $y=x-2 \sqrt{x}+2 \lg (1+\sqrt{x}) \frac{d y}{d x}=\frac{x}{x+\sqrt{x}}$

166) $y=\lg \frac{a+\sqrt{a^{2}-x^{2}}}{x}$

167) $y=\lg \left(a+b e^{x}\right)$

$$
\begin{aligned}
& n=\frac{-a}{x \sqrt{a^{2}-x^{2}}} \\
& n=\frac{b e^{x}}{a+b e^{x}}
\end{aligned}
$$

168) $y=\lg \frac{1}{\sqrt{1+x^{2}}}=-\frac{1}{2} \lg \left(1+x^{2}\right) \quad n=\frac{x}{1+x^{2}}$

169) $y=\lg \sqrt{2 a x-x^{2}}$

$n=\frac{a-x}{2 a x-x^{2}}$

170) $y=\lg \frac{1+x}{1-x}$

$$
n=\frac{2}{1-x^{2}}
$$

171) $y=\lg \sqrt{\frac{a+x}{a-x}}$

$n=\frac{a}{a^{2}-x^{2}}$

172) $y=\lg \frac{x}{\sqrt{1-x^{2}}}$

$n=\frac{1}{x\left(1-x^{2}\right)}$

173) $y=\frac{1}{x} \lg x$

$n=\frac{1-\lg x}{x^{2}}$

174) $y=\lg \frac{1+a \sqrt{x}}{1-a \sqrt{x}}$

$n=\frac{a \sqrt{x} \lg a}{\sqrt{x}}\left(\frac{1}{1-a^{2} \vee \bar{x}}\right)$

175) $y=x \sqrt{a^{2}+x^{2}}+\lg \left(x+\sqrt{a^{2}+x^{2}}\right) \frac{d y}{d x}=\frac{2 x^{2}+a^{2}+1}{\sqrt{a^{2}+x^{2}}}$

176) $y=\lg \left(a+x+\sqrt{2 a x+x^{2}}\right) \quad \frac{d y}{d x}=\frac{1}{\sqrt{2 a x+x^{2}}}$

177) $y=\frac{m}{2} \lg \left(x^{2}-a^{2}\right)+\frac{n}{2 a} \lg \frac{x-a}{x+a} \quad n=\frac{m x+n}{x^{2}-a^{2}}$

178) $y=\frac{1+x^{2}}{2} \lg \left(1+x^{2}\right)-\frac{x^{2}}{2} \quad n=x \lg \left(1+x^{2}\right)$

179) $y=\frac{3}{4} \lg \frac{x^{2}+1}{x^{2}-1}+\frac{1}{4} \lg \frac{x-1}{x+1}+\frac{1}{2} \operatorname{arctg} x \quad \frac{d y}{d x}=\frac{x^{2}-3 x}{x^{4}-1}$

180) $y=\lg (x-1)+3 \lg (x+1)+\lg \left(x^{2}+1\right)+5 \operatorname{arctg} x$

$$
\frac{d y}{d x}=\frac{6 x^{3}+3 x^{2}+2 x-7}{x^{4}-1}
$$


181) $y=\frac{1}{\sqrt{a}} \lg \frac{\sqrt{a+b x}-\sqrt{a}}{\sqrt{a+b x}+\sqrt{a}} \frac{d y}{d x}=\frac{1}{x \sqrt{a+b x}}$

182) $y=\frac{1}{x} \lg \frac{1+x}{1-x}$

$\eta=\frac{2}{x\left(1-x^{2}\right)}-\frac{1}{x^{2}} \lg \frac{1+x}{1-x}$

183) $y=\frac{1-x^{4}}{2 x^{2}} \lg \frac{1+x}{1-x}$

$n=\frac{1+x^{2}}{x^{2}}-\frac{x^{4}+1}{x^{3}} \lg \frac{1+x}{1-x}$

184) $y=\frac{x}{1-x} \lg x$

185) $y=\lg \sin x$

$n=\frac{1-x+\lg x}{(1-x)^{2}}$

$n=\operatorname{cotg} x$

186) $y=\lg \cos x$

$n=-\operatorname{tg} x$

187) $y=\lg \operatorname{tg} x$

$n=\frac{2}{\sin 2 x}$

188) $y=\lg \operatorname{cotg} x=\lg \operatorname{tg}\left(\frac{\pi}{2}-x\right) \quad "=-\frac{2}{\sin 2 x}$

189) $y=\lg \sin \left(\frac{x-a}{x}\right)=\lg \sin z \quad n=\frac{a}{x^{2}} \operatorname{cotg}\left(\frac{x-a}{x}\right)$

190) $y=\lg \left(\cos \frac{x}{2}\right)^{2}$

191) $y=\lg \sin \sqrt{a+b x}$

$n=-\operatorname{tg} \frac{x}{2}$

$n=\frac{b}{2 \sqrt{a+b x}} \operatorname{cotg} \sqrt{a+b x}$

192) $y=\lg \cos \sqrt{\frac{1}{x}}$

193) $y=\lg \left(\frac{a+b \operatorname{tg} x}{a-b \operatorname{tg} x}\right)$

$\eta=\frac{1}{2 x \sqrt{x}} \operatorname{tg} \sqrt{\frac{1}{x}}$

$$
n=\frac{2 a b}{a^{2}-\left(a^{2}+b^{2}\right) \sin ^{2} x}
$$

194) $y=\lg \operatorname{tg} x+\lg \cos x=\lg \sin x \quad \frac{d y}{d x}=\operatorname{cotg} x$

195) $y=\lg f(x)=\lg z$

Der Differentialquotient des natürlichen Logarithmus einer Funktion ist gleich einem Bruch, dessen Nenner die Funktion und dessen Zähler der Differentialquotient der Funktion ist.

Funktionen von der Form $y=f(u, v)=u v$, worin $u$ und $v$ Funktionen von $x$ sind, werden nach Formel (10) differentiiert. 
Es ist dann $\frac{\partial f}{\partial u}=v u^{v-1}, \quad \frac{\partial f}{\partial v}=u^{v} \lg u$, und

$$
\frac{d y}{d x}=v u^{v-1} \cdot \frac{d u}{d x}+u^{v} \lg u \cdot \frac{d v}{d x} .
$$

1. Beispiel: $y=(a x)^{m x} ; u=a x, v=m x ; \frac{d u}{d x}=a, \frac{d v}{d x}=m$,

$$
\frac{d y}{d x}=m(a x)^{m x}(1+\lg x+\lg a) .
$$

2. Beispiel: $y=(\sin x)^{\cos x} ; \quad \frac{d u}{d x}=\cos x, \frac{d v}{d x}=-\sin x$;

$$
\frac{d y}{d x}=(\sin x)^{\cos x-1}\left(\cos ^{2} x-\sin ^{2} x \lg \sin x\right) .
$$

Derartige Funktionen können etwas bequemer behandelt werden, wenn man beiderseits den Logarithmus nimmt und sich erinnert, dass eine Funktion von $y$, wenn $y$ selbst eine Funktion von $x$ ist, in der Weise nach $x$ differentiiert werden kann, dass man dieselbe zuerst nach $y$ differentiiert und diesen Differentialquotienten noch mit demjenigen von $y$ nach $x$ multipliciert.

3. Beispiel: $y=x^{x} ; \quad \lg y=x \lg x ; \frac{1}{y} \cdot \frac{d y}{d x}=1+\lg x$;

$$
\frac{d y}{d x}=(1+\lg x) y=x^{x}(1+\lg x) .
$$

4. Beispiel: $y=\left(\frac{a}{x}\right)^{x} ; \lg y=x(\lg a-\lg x)$;

$$
\frac{d y}{d x}=\left(\frac{a}{x}\right)^{x}(\lg a-\lg x-1) .
$$

5. Beispiel: $y=\left(\frac{1-x}{x}\right)^{\left(\frac{1-x}{x}\right)}=z^{z} ; \frac{d y}{d z}=z^{z}(1+\lg z)$,

$$
\frac{d z}{d x}=-\frac{1}{x^{2}}, \frac{d y}{d z}=-\left(\frac{1-x}{x}\right)^{\left(\frac{1-x}{x}\right)}\left[1+\lg \left(\frac{1-x}{x}\right)\right] \cdot \frac{1}{x^{2}}
$$

6. Beispiel: $y=\sqrt[x]{x} ; \frac{d y}{d x}=\frac{\sqrt[x]{x}}{x^{2}}(1-\lg x)$. 
7. Beispiel: $y=\sqrt{x / \frac{1}{x}} ; \frac{d y}{d x}=\left(\frac{1}{x}\right)^{\frac{1}{x}}\left(\frac{\lg x-1}{x^{2}}\right)$.

8.Beispiel: $y=(a+b x)^{\frac{1}{x}} ; \frac{d y}{d x}=\frac{(a+b x)^{\frac{1}{x}}}{x^{2}}\left(\frac{b x}{a+b x}-\lg (a+b x)\right)$.

9. Beispiel: $y=\left(\sqrt{\frac{a}{x}}\right)^{x} ; \frac{d y}{d x}=\frac{1}{2}\left(\frac{a}{x}\right)^{\frac{x}{2}}\left[\lg \left(\frac{a}{x}\right)-1\right]$.

10. Beispiel: $y=(\operatorname{arctg} x)^{x}$;

$$
\frac{d y}{d x}=(\operatorname{arctg} x)^{x}\left(\lg \operatorname{arctg} x+\frac{x}{\operatorname{arctg} x} \cdot \frac{1}{1+x^{2}}\right) .
$$

§ 6. Unentwickelte Funktionen.

Die Entwickelung der Differentialquotienten war seither an die Voraussetzung geknüpft, dass die Funktion explicite in der Form $y=f(x)$ gegeben sei. Sind dagegen die beiden Variabelen in der Weise mit einander verbunden, dass eine Funktion von beiden gleich Null gesetzt wird:

$$
f(x, y)=0,
$$

so ist (vgl. S. 3) $y$ eine unentwickelte oder implicite Funktion von $x$, deren explicite Form $y=\varphi(x)$ zwar durch $f(x, y)=0$ bestimmt ist, im allgemeinen aber nicht angegeben werden kann, weil uns die Mittel fehlen, $f(x, y)=0$ nach $y$ aufzulösen. Aus diesem Grunde muss das Verfahren, den Differentialquotienten $\mathrm{zu}$ bilden, in solchem Falle wesentlich modifiziert werden.

Die Formel (10), S. 16, lautete:

$$
y=f(u, v) ; \quad \frac{d y}{d x}=\frac{\partial y}{\partial u} \frac{d u}{d x}+\frac{\partial y}{\partial v} \frac{d v}{d x},
$$

wobei $u$ und $v$ Funktionen von $x$ waren. Setzt man insbesondere $y=0$, so heisst dies nur, dass die Funktionen $u, v$ die Gleichung $f(u, v)=0$ stets erfullen sollen. Dann wird $\frac{d y}{d x}=0$ und man erhält: 


$$
f(u, v)=0 ; \quad \frac{\partial f}{\partial u} \frac{d u}{d x}+\frac{\partial f}{\partial v} \frac{d v}{d x}=0 .
$$

Nimmt man ferner $u=x$ und setzt statt $v$ als neue Bezeiohnung $y$ ein, so hat man, da $\frac{d u}{d x}=1$ wird:

$$
\begin{gathered}
f(x, y)=0 ; \quad \frac{\partial f}{\partial x}+\frac{\partial f}{\partial y} \frac{d y}{d x}=0 \\
\frac{d y}{d x}=-\frac{\frac{\partial f}{\partial x}}{\frac{\partial f}{\partial y}} .
\end{gathered}
$$

1. Beispiel: $f=x^{2}+y^{2}-r^{2}=0$. Man findet direkt:

$$
2 x+2 y \frac{d y}{d x}=0, \text { und daraus } \frac{d y}{d x}=-\frac{x}{y},
$$

oder nach (22): $\frac{\partial f}{\partial x}=2 x, \frac{\partial f}{\partial y}=2 y, \frac{d y}{d x}=-\frac{x}{y}$.

Aus der hier darstellbaren entwickelten Form:

$$
y=\sqrt{r^{2}-x^{2}} \text { entsteht: } \frac{d y}{d x}=-\frac{x}{\sqrt{r^{2}-x^{2}}}=-\frac{x}{y} .
$$

2. Beispiel: $f=y^{n}-x^{p}=0 ; n y^{n-1} \frac{d y}{d x}-p x^{p-1}=0$,

$$
\frac{d y}{d x}=\frac{p x^{p-1}}{n y^{n-1}}=\frac{p}{n} \cdot \frac{x^{p-1}}{\left(x^{\frac{p}{n}}\right)^{n-1}}=\frac{p}{n} x^{\frac{p}{n}-1} .
$$

Nach der Formel (22) giebt es:

$$
\frac{\partial f}{\partial x}=-p x^{p-1}, \frac{\partial f}{\partial y}=n y^{n-1}, \frac{d y}{d x}=\frac{p x^{p-1}}{n y^{n-1}} \text { usw. }
$$

Will man die bei Funktionen in der entwickelten Form vorkommenden Nenner, Wurzelzeichen und dergl. vermeiden, so geht man zu den unentwickelten Formen uber. Statt $\frac{d y}{d x}$ wird gewöhnlich $y^{\prime}$ gesetzt.

3. Beispiel: $y=\frac{1}{\sqrt[3]{x^{2}}}, f=y^{3} x^{2}-1=0 ; 3 y^{2} y^{\prime} x^{2}+2 y^{3} x=0$, 
$y^{\prime}=-\frac{2 y}{3 x}$, oder: $y^{\prime}=-\frac{2}{3 \sqrt[3]{x^{5}}}$, wie auch durch direkte Ableitung aus der entwickelten Form.

4. Beispiel: $y=x \sqrt{\frac{a+b x}{a-b x}}, \frac{y^{2}}{x^{2}}=\frac{a+b x}{a-b x}$,

$$
\begin{gathered}
f=b x y^{2}+b x^{3}-a y^{2}+a x^{2}=0 \\
b y^{2}+2 b x y y^{\prime}+3 b x^{2}-2 a y y^{\prime}+2 a x=0, \\
y^{\prime}=\frac{b y^{2}+3 b x^{2}+2 a x}{2 a y-2 b x y} .
\end{gathered}
$$

5. Beispiel: $y=\sqrt{x+\sqrt{1+x^{2}}}$, oder $f=y^{4}-2 y^{2} x-1=0$; $4 y^{3} y^{\prime}-4 y x y^{\prime}-2 y^{2}=0,{ }^{\prime} y^{\prime}=\frac{y}{2\left(y^{2}-x\right)}=\frac{\sqrt{x+\sqrt{1+x^{2}}}}{2 \sqrt{1+x^{2}}}$.

Aufgaben.

196) $f=a x+b y+c=0 ; \quad \frac{\partial f}{\partial x}=a, \frac{\partial f}{\partial y}=b, \frac{d y}{d x}=-\frac{a}{b}$. 197) $f=\frac{x^{2}}{a^{2}}+\frac{y^{2}}{b^{2}}-1=0 ; \frac{\partial f}{\partial x}=\frac{2 x}{a^{2}}, \frac{\partial f}{\partial y}=\frac{2 y}{b^{2}}$, $\frac{d y}{d x}=-\frac{b^{2} x}{a^{2} y}=-\frac{b x}{a \sqrt{a^{2}-x^{2}}}$.

198) $y=\frac{(x+a)^{\frac{3}{2}}}{(x-a)^{\frac{1}{2}}} ; f=y^{2}(x-a)-(x+a)^{3}=0$ : $\frac{\partial f}{\partial x}=y^{2}-3(x+a)^{2}, \frac{\partial f}{\partial y}=2 y(x-a)$, $\frac{d y}{d x}=\frac{3(x+a)^{2}-y^{2}}{2 y(x-a)}=\frac{3}{2} \sqrt{\frac{x+a}{x-a}}-\frac{1}{2} \sqrt{\left(\frac{x+a}{x-a}\right)^{2}}$. 199) $f=a^{x-y}-x^{y}=0 ; \quad \frac{\partial f}{\partial x}=a^{x-y} \lg a-y x^{y-1}$, $\frac{\partial f}{\partial y}=-a^{x-y} \lg a-x^{y} \lg x, \frac{d y}{d x}=\frac{x \lg a-y}{x \lg (a x)}$. 2.200) $f=\sin x-\cos y=0, \frac{d y}{d x}=-\frac{\cos x}{\sin y}=-1$. 
201) $f=e^{x+y}-a^{x}=0 ; \quad \frac{\partial f}{\partial x}=e^{x+y}-a^{x} \lg a, \quad \frac{\partial f}{\partial y}=e^{x+y}$ $\frac{d y}{d x}=-1+\lg a$.

202) $f=e^{a x+b y}-c=0 ; \quad \frac{\partial f}{\partial x}=a e^{a x+b y}, \quad \frac{\partial f}{\partial y}=b e^{a x+b y}$, $\frac{d y}{d x}=-\frac{a}{b}$. Aus der einfacheren Form: $a x+b y=\lg c$ folgt das gleiche Resultat.

203) $f=\left(e^{x}-1\right)\left(e^{y}-1\right)-1=0, \frac{\partial f}{\partial x}=e^{x}\left(e^{y}-1\right)$ $\frac{\partial f}{\partial y}=e^{y}\left(e^{x}-1\right), \quad \frac{d y}{d x}=-\frac{e^{y}-1}{e^{x}-1} \cdot \frac{e^{x}}{e^{y}}=-\frac{e^{x}}{e^{y}}\left(e^{y}-1\right)^{2}$. 204) $f=a y-e^{\sqrt{b-x}}=0 ; \quad \frac{\partial f}{\partial x}=\frac{e^{\sqrt{b-x}}}{2 \sqrt{b-x}}, \frac{\partial f}{\partial y}=a$, $\frac{d y}{d x}=\frac{-y}{2 \sqrt{b-x}}$.

205) $f=\cos x-x \cos y=0 ; \quad \frac{\partial f}{\partial x}=-\sin x-\cos y$, $\frac{\partial f}{\partial y}=x \sin y, \quad \frac{d y}{d x}=\frac{\sin x+\cos y}{x \sin y}$.

206) $f=y(x+\sin x)=0$; man erkennt leicht, dass die so gestellte Aufgabe uns lediglich auf $d x=0$ oder $d y=0$ führen kann. Denn das $f$ verschwindet nur, wenn entweder $y=0$ oder $x+\sin x=0$ ist. 207) $f=x \sin (x-y)-(x+y)=0 ; \frac{\partial f}{\partial x}=\sin (x-y)+x \cos (x-y)-1$, $\frac{\partial f}{\partial y}=-x \cos (x-y)-1, \frac{d y}{d x}=\frac{\sin (x-y)+x \cos (x-y)-1}{x \cos (x-y)+1}$.

In den folgenden Aufgaben sollen die partiellen Differentialquotienten nicht getrennt entwickelt, sondern es soll unmittelbar nach der Formel differentiiert werden:

$$
f(x, y)=0, \quad \frac{\partial f}{\partial x}+\frac{\partial f}{\partial y} y^{\prime}=0 .
$$


208) $f=y^{2}-2 p x=0 ; \quad 2 y y^{\prime}-2 p=0, y^{\prime}=\frac{p}{y}$.

209) $f=\sqrt{\frac{x}{a}}+\sqrt{\frac{y}{b}}-c=0 ; \frac{1}{2 a} \sqrt{\frac{a}{x}}+\frac{1}{2 b} \sqrt{\frac{b}{y}} y^{\prime}=0$, $y^{\prime}=-\sqrt{\frac{b y}{a x}}$.

210) $f=\arcsin \frac{x}{y}-\lg x=0 ; \frac{1}{\sqrt{y^{2}-x^{2}}}-\frac{x}{y \sqrt{y^{2}-x^{2}}} y^{\prime}-\frac{1}{x}=0$, $y^{\prime}=\frac{x y-y \sqrt{y^{2}-x^{2}}}{x^{2}}$.

211) $f=y^{2}+x^{2}-(a x+b)^{2}=0 ; y y^{\prime}+x-a(a x+b)=0$. 212) $f=(x-\alpha)^{2}+(y-\beta)^{2}+2 \lambda x y=0$;

$(x-\alpha+\lambda y)+(y-\beta+\lambda x) y^{\prime}=0$.

213) $f=y^{2}\left(x^{2}-1\right)-a x^{2}=0 ; y\left(x^{2}-1\right) y^{\prime}+x\left(y^{2}-a\right)=0$.

214) $f=(a x+b y+c)(\alpha x+\beta y+\gamma)-m x^{2}-n y^{2}=0$;

$\left(a+b y^{\prime}\right)(\alpha x+\beta y+\gamma)+\left(\alpha+\beta y^{\prime}\right)(a x+b y+c)$

$-2 m x-2 n y y^{\prime}=0$.

215) $f=\left(x^{2}+y^{2}-1\right)(a x+b y)-m x^{2}-n y^{2}=0$;

$2\left(x+y y^{\prime}\right)(a x+b y)+\left(a+b y^{\prime}\right)\left(x^{2}+y^{2}-1\right)$

$-2 m x-2 n y y^{\prime}=0$.

216) $f=(1-a x)\left(x^{2}+y^{2}\right)-4=0$;

$-a\left(x^{2}+y^{2}\right)+2\left(x+y y^{\prime}\right)(1-a x)=0$.

217) $f=\left(1+x+\frac{1}{y}\right)\left(1+y+\frac{1}{x}\right)=0$;

$\left(1-\frac{y^{\prime}}{y^{2}}\right)\left(1+y+\frac{1}{x}\right)+\left(y^{\prime}-\frac{1}{x^{2}}\right)\left(1+x+\frac{1}{y}\right)=0$.

218) $f=\left(x^{2}+y^{2}-a x\right)^{2}-a^{2}\left(x^{2}+y^{2}\right)=0$;

$\left(x^{2}+y^{2}-a x\right)\left(2 x+2 y y^{\prime}-a\right)-a^{2}\left(x+y y^{\prime}\right)=0$.

219) $f=x^{2} y^{2}-\left(a^{2}-x^{2}\right)(y+b)^{2}=0$;

$x y^{2}+x^{2} y y^{\prime}+x(y+b)^{2}-(y+b) y^{\prime}\left(a^{2}-x^{2}\right)=0$.

220) $f=a^{x}-e^{x-y}=0 ; a^{x} \lg a-e^{x-y}+e^{x-y} y^{\prime}=0, y^{\prime}=1-\lg a$.

Einfacher so: $x \lg a=x-y ; y^{\prime}=1-\lg a$.

221) $f=y^{2}-2 y e^{x}+2 x \lg y=0$;

$y y^{\prime}-e^{x}\left(y+y^{\prime}\right)+\lg y+x y^{-1} y^{\prime}=0$. 
222) $f=\sin x-\sin (2 y-x)=0$;

$$
\cos x+\cos (2 y-x)-2 y^{\prime} \cos (2 y-x)=0 \text {. }
$$

223) $f=y \sin x+x^{2} y^{2}+a \cos y+b=0$;

$y \cos x+y^{\prime} \sin x+2 x y\left(y+x y^{\prime}\right)-a y^{\prime} \sin y=0$.

224) $f=e^{x} \cos y-e^{y} \sin x=0$;

$e^{x} \cos y-e^{y} \cos x-\left(e^{x} \sin y+e^{y} \sin x\right) y^{\prime}=0$.

225) $f=\sin x+\sin y+\sin (x+y)=0$;

$$
y^{\prime}=-\frac{\cos x+\cos (x+y)}{\cos y+\cos (x+y)}
$$

§ 7. Funktionen von der Form:

$$
x=\varphi(t), y=\psi(t) .
$$

Oft ist es zweckmässig, $y$ in der Weise von $x$ abhängig zu machen, dass man beide als Funktionen einer dritten Variabelen darstellt, indem man setzt:

$$
x=\varphi(t), y=\psi(t) .
$$

Kann $t$ daraus eliminiert werden, so erhält man die entsprechende direkte Beziehung zwischen $x$ und $y$ wieder. So geht z. B. $x=r \cos t, y=r \sin t$ leicht in $f=x^{2}+y^{2}-r^{2}$ $=0$ iber.

$\mathrm{Da} t$ in diesen Formen die eigentliche unabhängige Variabele ist, so legen wir ihr die besonderen Werte $t$ und $t_{1}$ bei und erhalten die entsprechenden Funktionswerte:

$$
\begin{array}{ll}
x=\varphi(t) & x_{1}=\varphi\left(t_{1}\right) \\
y=\psi(t) & y_{1}=\psi\left(t_{1}\right),
\end{array}
$$

die wir zu folgender Identität zusammensetzen:

$$
\frac{y_{1}-y}{x_{1}-x}=\frac{\frac{y_{1}-y}{t_{1}-t}}{\frac{x_{1}-x}{t_{1}-t}}
$$

Da nun der Übergang von $t_{1}$ in $t$ auch diejenigen von $x_{1}$ in $x$ und von $y_{1}$ in $y$ zur Folge hat, so gehen alle Differenzen- 
quotienten in (A) gleichzeitig in Differentialquotienten über, und wir erhalten:

$$
\frac{d y}{d x}=\frac{\frac{d y}{d t}}{\frac{d x}{d t}} .
$$

Unter der Gestalt: $\frac{d y}{d t}=\frac{d y}{d x} \cdot \frac{d x}{d t}$, oder: $\frac{d y}{d x}=\frac{d y}{d t} \cdot \frac{d t}{d x}$ ist nur eine Wiederholung von Formel (6), S. 11.

1. Beispiel: $x=a \cos t, y=b \sin t ; \quad \frac{d x}{d t}=-a \sin t$, $\frac{d y}{d t}=b \cos t ; \frac{d y}{d x}=-\frac{b}{a} \operatorname{cotg} t$. Wird $t$ eliminiert, so erhält man die Gleichung: $f=\frac{x^{2}}{a^{2}}+\frac{y^{2}}{b^{2}}-1=0$, oder: $y=\frac{b}{a} \sqrt{a^{2}-x^{2}}$, und daraus für den Differentialquotienten die beiden Formen:

$$
\frac{d y}{d x}=-\frac{b^{2} x}{a^{2} y}, \text { oder } \frac{d y}{d x}=\frac{-b x}{a \sqrt{a^{2}-x^{2}}} .
$$

Da nun $\operatorname{cotg} t=\frac{b x}{a y}$ und auch $=\frac{x}{\sqrt{a^{2}-x^{2}}}$ ist, so lassen die 3 Formen des Differentialquotienten sich auf einander zurückfuhren.

2. Beispiel: $x=t \cos \alpha, y=t \sin \alpha+b ; \frac{d x}{d t}=\cos \alpha$, $\frac{d y}{d t}=\sin \alpha ; \frac{d y}{d x}=\operatorname{tg} \alpha$. Wird $t$ eliminiert, so entsteht: $y=x \operatorname{tg} \alpha+b$ und daraus: $\frac{d y}{d x}=\operatorname{tg} \alpha$.

\section{Aufgaben.}

226) $y=a t$

$$
x=a(1-t)
$$

227) $y=\frac{a}{a-t}$

$$
x=\frac{b}{b-t}
$$

$$
\frac{d y}{d x}=-1
$$

$$
n=\frac{a(b-t)^{2}}{b(a-t)^{2}}
$$


228) $y=\frac{1-t}{1+t}$

$$
x=\frac{2 t}{1+t}
$$

229) $y=\frac{t^{2}}{2}$

$$
x=\frac{2}{3} \sqrt{2 t^{3}}
$$

230) $y=\left(e^{a t}-1\right)^{3}$

$$
x=\left(e^{a t}-1\right)^{2}
$$

231) $y=\frac{4(a-t)^{3}}{a^{2} t^{2}}$

$$
x=\frac{3 a-2 t}{a t}
$$

232) $y=\sin ^{2} t$

$$
x=\sin 2 t
$$

233) $y=b \sin ^{2} t$

$$
x=a \cos ^{2} t
$$

234) $y=1-\cos t$

$$
x=t-\sin t
$$

235) $y=a \cos ^{2} \frac{b t}{2}$

$$
x=b \operatorname{tg} \frac{b t}{2}
$$

2 236) $y=\operatorname{tg} t$

$$
x=\frac{1}{\cos ^{2} t}
$$

237) $y=\sin t-t \cos t$

$$
x=\cos t+t \sin t
$$

238) $y=\frac{1}{2} \sin t+\sin \frac{t}{2}$

$$
x=\frac{1}{2} \cos t+\cos \frac{t}{2}
$$

$$
\text { 2239) } \begin{aligned}
y & =t-\cos t \\
x & =t-\sin t
\end{aligned}
$$

$$
\frac{d y}{d x}=-1
$$

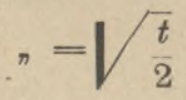

$$
n=\frac{3}{2}\left(e^{a t}-1\right)
$$

$$
"=\frac{4(a-t)^{2}(2 a+t)}{3 a^{2} t}
$$

$\eta=\frac{1}{2} \operatorname{tg} 2 t$

$n=-\frac{b}{a}$

$n=\operatorname{cotg} \frac{t}{2}$

$n=-\frac{a}{b} \sin b t \cos ^{2} \frac{b t}{2}$ $n=\operatorname{tg} t$

$n=-\operatorname{cotg} \frac{3}{4} t$

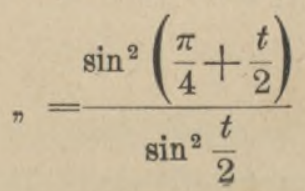


240) $\}=\frac{a \sin t}{1+b \cos t}$

$$
x=\frac{c \cos t}{1+b \cos t}
$$

$$
\frac{d y}{d x}=-\frac{a b+a \cos t}{c \sin t}
$$

241) $y=\sin t(\cos 2 t)^{\frac{1}{2}}$

$$
x=\cos t(\cos 2 t)^{\frac{1}{2}}
$$$$
n=-\operatorname{cotg} 3 t
$$

242) $y=\frac{\sin ^{3} t}{(\cos 2 t)^{\frac{1}{2}}}$

$$
x=\frac{\cos ^{3} t}{(\cos 2 t)^{\frac{1}{2}}}
$$$$
n=-\operatorname{tg} 3 t
$$

243) $y=2 \sin t-\sin 2 t$

$$
x=2 \cos t-\cos 2 t
$$$$
{ }_{n}=\operatorname{tg} \frac{3}{2} t
$$

244) $y=(a+r) \sin t-a \sin \left(\frac{a+r}{a}\right) t$

$$
\begin{aligned}
& y=(a+r) \sin t-a \sin \left(\frac{a+r}{a}\right) t \\
& x=(a+r) \cos t-a \cos \left(\frac{a+r}{a}\right) t
\end{aligned} \quad \frac{d y}{d x}=\operatorname{tg} \frac{2 a+r}{2 a} t
$$

245) $y=\arcsin \frac{t}{\sqrt{1+t^{2}}}$

$$
x=\arccos \frac{1}{\sqrt{1+t^{2}}}
$$

$$
n=1 \text {. }
$$

\section{§ 8. Differentialquotienten hŏherer Ordnung.}

Entwickelte Funktionen. Der Differentialquotient von $y=f(x)$ ist wieder eine Funktion von $x$ und kann deshalb den nämlichen Operationen unterworfen werden, durch welche er selbst aus der gegebenen Funktion hervorgegangen ist. Wir können also setzen:

$$
\begin{gathered}
\frac{d f^{\prime}(x)}{d x}=\lim \frac{f^{\prime}(x+\Delta x)-f^{\prime}(x)}{\Delta x} \\
=\operatorname{m} \frac{1}{\Delta x}\left[\frac{f(x+2 \Delta x)-f(x+\Delta x)}{\Delta x}-\frac{f(x+\Delta x)-f(x)}{\Delta x}\right] \\
=\lim \frac{f(x+2 \Delta x)-2 f(x+\Delta x)+f(x)}{\Delta x^{2}}
\end{gathered}
$$


den Zähler des letzten Ausdruckes schreiben wir $\Delta(\Delta f)=\Delta^{2} f$ $=\Delta(\Delta y)=\Delta^{2} y$ und erhalten:

$$
\frac{d f^{\prime}(x)}{d x}=\lim \frac{\Delta^{2} f}{\Delta x^{2}}=\lim \frac{\Delta^{2} y}{\Delta x^{2}} .
$$

Wir gebrauchen hierfür die Bezeichnung $f^{\prime \prime}(x)$ oder $y^{\prime \prime}$, so dass:

Ebenso setzen wir

$$
y^{\prime \prime}=f^{\prime \prime}(x)=\frac{d f^{\prime}(x)}{d x}=\frac{d}{d x}\left(\frac{d f}{d x}\right)=\frac{d^{2} f}{d x^{2}} .
$$

$$
y^{\prime \prime \prime}=f^{\prime \prime \prime}(x)=\frac{d f^{\prime \prime}(x)}{d x}=\frac{d}{d x}\left(\frac{d^{2} f}{d x^{2}}\right)=\frac{d^{2}}{d x^{2}}\left(\frac{d f}{d x}\right)=\frac{d^{3} f}{d x^{3}},
$$

u. s. f. Diese Ausdrücke

$y^{\prime \prime}, y^{\prime \prime \prime}, y^{\prime \prime \prime \prime}, \ldots y^{(n)}$ oder: $f^{\prime \prime}(x), f^{\prime \prime \prime}(x), \quad f^{\prime \prime \prime \prime}(x), \ldots f^{(n)}(x)$ heissen der zweite, der dritte, ... der $n^{\text {te }}$ Differentialquotient und generell: Differentialquotienten höherer Ordnungen.

1. Beispiel: $y=x^{p} ; \frac{d y}{d x}=p x^{p-1}, \frac{d^{2} y}{d x^{2}}=p(p-1) x^{p-2}$,

$$
\begin{aligned}
& \frac{d^{3} y}{d x^{8}}=p(p-1)(p-2) x^{p-3}, \ldots \\
& \frac{d^{n} y}{d x^{n}}=p(p-1) \ldots(p-n+1) x^{p-n} .
\end{aligned}
$$

2. Beispiel: $y=\sin x ; y^{\prime}=\cos x, y^{\prime \prime}=-\sin x$, $y^{\prime \prime \prime}=-\cos x, y^{\prime \prime \prime \prime}=\sin x$, u. s. w.

3. Beispiel: $f(x)=e^{x} ; f^{\prime}(x)=e^{x}, f^{\prime \prime}(x)=e^{x}$, usw.

A u f gaben.

246) $y=x^{3}$

$$
\frac{d^{2} y}{d x^{2}}=6 x
$$

247) $y=(a-b x)^{4}$

$$
\begin{array}{ll}
\text { 247) } y=(a-b x)^{4} & "=12 b^{2}(a-b x)^{2} \\
\text { 248) } y=x^{4}-2 x^{3}+5 x^{2}+2 & "=12 x^{2}-12 x+10 \\
\text { 249) } y=\sqrt[3]{x^{2}} & n=-\frac{2}{9 \sqrt[3]{x^{4}}}
\end{array}
$$

249) $y=\sqrt[3]{x^{2}}$

250) $y=\sqrt{a^{2}-x^{2}}$

$n=-\frac{a^{2}}{\sqrt{\left(a^{2}-x^{2}\right)^{3}}}$

251) $y=\sqrt{2 p x}$

$\Rightarrow=-\frac{p^{2}}{\sqrt{(2 p x)^{3}}}$ 
252) $y=\frac{1}{\sqrt[3]{a+b x}}$

253) $y=x+\sqrt[3]{(x-a)^{5}}$

254) $y=x(a-x)$

255) $y=x+\frac{1}{x}$

256) $y=\frac{x}{(a-x)^{2}}$

257) $y=e^{m x}$

258) $y=a^{m x}$

259) $y=e^{\varphi(x)}$

260) $y=e^{-x^{2}}$

261) $y=\lg \left(1+x^{2}\right)$

262) $y=x \lg x$

263) $y=x^{2} \lg x$

264) $y=\frac{\lg x}{x}$

265) $y=\sin (a-2 x)$

266) $y=\frac{1-\cos x}{\sin x}$

267) $y=\arccos x$

268) $y=x e^{\sin x}$

269) $y=\left(\frac{1}{x}\right)^{x}$

270) $y=\arcsin \frac{x}{\sqrt{1+x^{2}}}$

271) $y=\arcsin \frac{1-x^{2}}{1+x^{2}}$

Dölp, Aufgaben.

$$
\frac{d^{2} y}{d x^{2}}=\frac{4 b^{2}}{9 \sqrt[3]{(a+b x)^{7}}}
$$

10

$$
\begin{aligned}
& n=-2 \\
& r=\frac{2}{x^{3}}
\end{aligned}
$$$$
n=\frac{10}{9 \sqrt[3]{x-a}}
$$

$n=\frac{4 a+2 x}{(a-x)^{4}}$

$n=m^{2} e^{m x}$

$n=a^{m x}(m \lg a)^{2}$

$n=e^{\varphi(x)}\left[\left(\varphi^{\prime}(x)\right)^{2}+\varphi^{\prime \prime}(x)\right]$

$\eta=2\left(2 x^{2}-1\right) e^{-x^{2}}$

$\eta=\frac{2\left(1-x^{2}\right)}{\left(1+x^{2}\right)^{2}}$

$n=\frac{1}{x}$

" $=3+2 \lg x$

$"=\frac{2 \lg x-3}{x^{3}}$

$\eta=-4 \sin (a-2 x)$

$=\frac{\operatorname{tg} \frac{x}{2}}{2 \cos ^{2} \frac{x}{2}}$

$"=-\frac{x}{\sqrt{\left(1-x^{2}\right)^{3}}}$

$n=e^{\sin x}\left(2 \cos x+x \cos ^{2} x-x \sin x\right)$

$n=\left(\frac{1}{x}\right)^{x}\left(-\frac{1}{x}+(1+\lg x)^{2}\right)$

$"=-\frac{2 x}{\left(1+x^{2}\right)^{2}}$

$n=\frac{4 x}{\left(1+x^{2}\right)^{2}}$ 
272) $y=\operatorname{arctg} \frac{x}{a}$

273) $y=x^{6}$

274) $y=\frac{1}{x}$

275) $y=\sqrt{x}$

276) $y=\lg (a+b x)$

277) $y=\sin (n x)$

$$
\begin{aligned}
\frac{d^{2} y}{d x^{2}} & =-\frac{2 a x}{\left(x^{2}+a^{2}\right)^{2}} \\
y^{\prime \prime \prime} & =6 \cdot 5 \cdot 4 x^{8} \\
n & =-\frac{1 \cdot 2 \cdot 3}{x^{4}} \\
n & =\frac{1 \cdot 3}{8 \sqrt{x^{5}}} \\
n & =\frac{2 b^{3}}{(a+b x)^{8}} \\
n & =-n^{8} \cos (n x) .
\end{aligned}
$$

Ist $y=f(z)$ und $z=\varphi(x)$, so ist $\frac{d y}{d x}=\frac{d y}{d z} \cdot \frac{d z}{d x}$. Wird auf beiden Seiten nochmals differentiiert, so entsteht nach (6), S.11:

$$
\frac{d^{2} y}{d x^{2}}=\frac{d^{2} y}{d z^{2}}\left(\frac{d z}{d x}\right)^{2}+\frac{d^{2} z}{d x^{2}} \cdot \frac{d y}{d z}
$$

Eine weitere Differentiation ergiebt:

$$
\begin{gathered}
\frac{d^{3} y}{d x^{3}}=\frac{d^{3} y}{d z^{3}}\left(\frac{d z}{d x}\right)^{3}+2 \frac{d z}{d x} \cdot \frac{d^{2} z}{d x^{2}} \cdot \frac{d^{2} y}{d z^{2}}+\frac{d^{3} z}{d x^{3}} \cdot \frac{d y}{d z}+\frac{d^{2} y}{d z^{2}} \cdot \frac{d z}{d x} \cdot \frac{d^{2} z}{d x^{2}}, \\
\frac{d^{3} y}{d x^{3}}=\frac{d^{3} y}{d z^{3}}\left(\frac{d z}{d x}\right)^{8}+3 \frac{d^{2} y}{d z^{2}} \cdot \frac{d z}{d x} \cdot \frac{d^{2} z}{d x^{2}}+\frac{d y}{d z} \cdot \frac{d^{3} z}{d x^{3}} .
\end{gathered}
$$

Beispiel: $y=\left(a+b x^{2}\right)^{3}=z^{3}, z=a+b x^{2}$;

$\frac{d y}{d z}=3 z^{2}, \frac{d^{2} y}{d z^{2}}=6 z, \frac{d^{3} y}{d z^{3}}=6, \frac{d z}{d x}=2 b x, \frac{d^{2} z}{d x^{2}}=2 b, \frac{d^{3} z}{d x^{3}}=0$ $\frac{d^{3} y}{d x^{8}}=24 b^{2} x\left(3 a+5 b x^{2}\right)$.

Unentwickelte Funktionen. Implicite ist $y$ eine Funktion von $x$, wenn eine Gleichung $f(x, y)=0$ den Zusammenhang zwischen $x$ und $y$ ausspricht. Wir leiten den Differentialquotienten $\frac{d y}{d x}$ aus der Gleichung: $\frac{\partial f}{\partial x}+\frac{\partial f}{\partial y} \cdot \frac{d y}{d x}=0 \mathrm{ab}$. Wird nun der partielle Differentialquotient $\frac{\partial f}{\partial x}$ nochmals partiell nach $x$ differentiiert, so erhält man den zweiten partiellen Differentialquotienten der Funktion genommen nach $x$ und be- 
zeichnet ihn durch $\frac{\partial^{2} f}{\partial x^{2}}$. Ebenso wird durch $\frac{\partial^{2} f}{\partial y^{2}}$ derjenige Differentialquotient bezeichnet, welcher entsteht, wenn man $f$ zweimal partiell nach $y$ differentiiert. In ganz analoger Weise haben wir unter $\frac{\partial^{2} f}{\partial y \partial x}$ d. h. $\frac{\partial\left(\frac{\partial f}{\partial x}\right)}{\partial y}$ denjenigen Differentialquotienten zu verstehen, der dadurch gewonnen wird, dass man $f$ zuerst partiell nach $x$ und dann partiell nach $y$ differentiiert. Von ihm unterscheidet sich der andere Differentialquotient $\frac{\partial\left(\frac{\partial f}{\partial y}\right)}{\partial x}$ oder $\frac{\partial^{2} f}{\partial x \partial y}$ dadurch, dass bei seiner Ableitung die Funktion $f(x, y)$ zuerst nach $y$ und dann nach $x$ partiell differentiiert wird.

$$
\begin{array}{ll}
\text { Beispiel: } f=x^{3}+3 x^{2} y-y^{3}=0 \\
\begin{array}{ll}
\frac{\partial f}{\partial x}=3 x^{2}+6 x y & \frac{\partial f}{\partial y}=3 x^{2}-3 y^{2} \\
\frac{\partial^{2} f}{\partial x^{2}}=6 x+6 y & \frac{\partial^{2} f}{\partial y^{2}}=-6 y \\
\frac{\partial^{2} f}{\partial x \partial y}=6 x & \frac{\partial^{2} f}{\partial y \partial x}=6 x .
\end{array}
\end{array}
$$

In diesem Beispiele ist $\frac{\partial^{2} f}{\partial x \partial y}=\frac{\partial^{2} f}{\partial y \partial x}$. Es soll nun nachgewiesen werden, dass diese zwei Differentialquotienten, soweit sie mit $x$ und $y$ sich stetig ändern, einander identisch gleich sind. Setzt man in:

$$
\frac{\partial f}{\partial x}=\lim \frac{f\left(x_{1}, y\right)-f(x, y)}{x_{1}-x}
$$

an die Stelle von $y$ die besonderen Werte $y$ und $y_{1}$, bildet den Differenzenquotienten und geht zur Grenze über, so erhält man:

$$
\frac{\partial\left(\frac{\partial f}{\partial x}\right)}{\partial y}=\lim \frac{\frac{f\left(x_{1}, y_{1}\right)-f\left(x, y_{1}\right)}{x_{1}-x}-\frac{f\left(x_{1}, y\right)-f(x, y)}{x_{1}-x}}{y_{1}-y}
$$


wobei durch lim der gleichzeitige Übergang von $x_{1}$ zu $x$ und von $y_{1}$ zu $y$ angezeigt ist. Ebenso entsteht aus:

$$
\frac{\partial f}{\partial y}=\lim \frac{f\left(x, y_{1}\right)-f(x, y)}{y_{1}-y}
$$

durch Substitution der besonderen Werte $x$ und $x_{1}$ und duroh analoge Differenz- und Quotientenbildung der Ausdruck:

$$
\frac{\partial\left(\frac{\partial f}{\partial y}\right)}{\partial x}=\lim \frac{\frac{f\left(x_{1}, y_{1}\right)-f\left(x_{1}, y\right)}{y_{1}-y}-\frac{f\left(x, y_{1}\right)-f(x, y)}{y_{1}-y}}{x_{1}-x}
$$

in dem sich das Zeichen lim wiederum auf den gleichzeitigen Übergang von $x_{1}$ zu $x$ und von $y_{1}$ zu $y$ bezieht. Da jeder der beiden unter dem Zeichen lim stehenden Ausdrücke sich auf die Form bringen lässt:

$$
\frac{f\left(x_{1}, y_{1}\right)-f\left(x_{1}, y\right)-f\left(x, y_{1}\right)+f(x, y)}{\left(x_{1}-x\right)\left(y_{1}-y\right)},
$$

so müssen die angezeigten gleichzeitigen Grenzübergänge auch zu gleichen Resultaten führen, $d$. h. es muss sein:

$$
\frac{\partial\left(\frac{\partial f}{\partial x}\right)}{\partial y}=\frac{\partial\left(\frac{\partial f}{\partial y}\right)}{\partial x} \text { oder: } \frac{\partial^{2} f}{\partial y \partial x}=\frac{\partial^{2} f}{\partial x \partial y} .
$$

Doch ist zu bemerken, dass die ganze Beweisführung nur für ein solches Werthegebiet von $x$ und $y$ gilt, in welchem für beliebige Annäherung des $x_{1}$ an $x$ und des $y_{1}$ an $y$ der Grenzwert

$$
\lim \frac{f\left(x_{1}, y_{1}\right)-f\left(x_{1}, y\right)-f\left(x, y_{1}\right)+f(x, y)}{\left(x_{1}-x\right)\left(y_{1}-y\right)}
$$

endlich und stetig variabel ist.

Soll nun auf beiden Seiten der Gleichung $\frac{\partial f}{\partial x}+\frac{\partial f}{\partial y} \cdot \frac{d y}{\partial x}=0$ nochmals und zwar total nach $x$ differentiiert werden, so muss man sich erinnern, dass $\frac{\partial f}{\partial x}$ und $\frac{\partial f}{\partial y}$ die beiden Variabelen $x$ und $y$ enthalten, und dass daher die Regel Anwendung findet: Wenn $F(x, y)=0$ ist, so wird: 


$$
\frac{d F(x, y)}{d x}=\frac{\partial F}{\partial x}+\frac{\partial F}{\partial y} \cdot \frac{d y}{d x} .
$$

Hiernach erhält man:

$$
\frac{\partial^{2} f}{\partial x^{2}}+\frac{\partial^{2} f}{\partial x \partial y} \cdot \frac{d y}{d x}+\left(\frac{\partial^{2} f}{\partial x \partial y}+\frac{\partial^{2} f}{\partial y^{2}} \cdot \frac{d y}{d x}\right) \frac{d y}{d x}+\frac{d^{2} y}{d x^{2}} \cdot \frac{\partial f}{\partial y}=0
$$

und daraus gewinnt man durch die Substitution $\frac{d y}{d x}=-\frac{\partial f}{\partial x}: \frac{\partial f}{\partial y}$ und die geeigneten Reduktionen schliesslich die Formel:

$$
\frac{d^{2} y}{d x^{2}}=-\frac{\frac{\partial^{2} f}{\partial x^{2}}\left(\frac{\partial f}{\partial y}\right)^{2}-2 \frac{\partial^{2} f}{\partial x \partial y} \cdot \frac{\partial f}{\partial x} \cdot \frac{\partial f}{\partial y}+\frac{\partial^{2} f}{\partial y^{2}}\left(\frac{\partial f}{\partial x}\right)^{2}}{\left(\frac{\partial f}{\partial y}\right)^{8}}
$$

Da die bisherigen Erläuterungen ausreichen, um auch die noch höheren Differentialquotienten einer unentwickelten Funktion herzuleiten, so unterlassen wir es, die bezüglichen allgemeinen Formeln hier besonders aufzustellen

1. Beispiel: $f=y^{2}+b x^{2}-2 a y+a x-b=0$;

$$
\begin{array}{cc}
\frac{\partial f}{\partial x}=2 b x+a & \frac{\partial f}{\partial y}=2 y-2 a \\
\frac{\partial^{2} f}{\partial x^{2}}=2 b & \frac{\partial^{2} f}{\partial y^{2}}=2 \\
\frac{\partial^{2} f}{\partial x \partial y}=0 & \frac{\partial^{2} f}{\partial y \partial x}=0 \\
\frac{d^{2} y}{d x^{2}}=-\frac{4 b(y-a)^{2}+(2 b x+a)^{2}}{4(y-a)^{3}} .
\end{array}
$$

2. Beispiel: $f=e^{a x-b y}-c=0$;

$$
\begin{array}{ll}
\frac{\partial f}{\partial x}=a e^{a x-b y} & \frac{\partial f}{\partial y}=-b e^{a x-b y} \\
\frac{\partial^{2} f}{\partial x^{2}}=a^{2} e^{a x-b y} & \frac{\partial^{2} f}{\partial y^{2}}=b^{2} e^{a x-b y} \\
\frac{\partial^{2} f}{\partial x \partial y}=-a b e^{a x-b y} & \frac{\partial^{2} f}{\partial y \partial x}=-a b e^{a x-b y} \\
\frac{d^{2} y}{d x^{2}}=0 .
\end{array}
$$


Die Form $a x-b y=\lg c$ ermöglicht einfacher das gleiche Resultat.

\section{A u f g a ben:}

Statt der vollständigen Lösungen geben wir hier nur die Teile, aus welchen jene nach (24), S. 53, zusammengesetzt werden.

278) $f=x^{3}-x^{2} y+y^{5}=0$

$\frac{\partial f}{\partial x}=3 x^{2}-2 x y$

$\frac{\partial f}{\partial y}=5 y^{4}-x^{2}$

$\frac{\partial^{2} f}{\partial x^{2}}=6 x-2 y$

$\frac{\partial^{2} f}{\partial y^{2}}=20 y^{3}$

$\frac{\partial^{2} f}{\partial y \partial x}=-2 x$.

279) $f=\left(x^{2}+y^{2}\right)^{2}-a^{2}\left(x^{2}-y^{2}\right)=0$

$\frac{\partial f}{\partial y}=2 y\left(2 x^{2}+2 y^{2}+a^{2}\right)$

$\frac{\partial f}{\partial x}=2 x\left(2 x^{2}+2 y^{2}-a^{2}\right)$

$\frac{\partial^{2} f}{\partial y^{2}}=12 y^{2}+4 x^{2}+2 a^{2}$

$\frac{\partial^{2} f}{\partial x^{2}}=12 x^{2}+4 y^{2}-2 a^{2}$

$\frac{\partial^{2} f}{\partial x \partial y}=8 x y$.

280) $f=e^{\sin x}-x e^{\sin y}=0$

$\frac{\partial f}{\partial y}=-x e^{\sin y} \cos y$

$\frac{\partial f}{\partial x}=e^{\sin x} \cos x-e^{\sin y} \quad \frac{\partial^{2} f}{\partial y^{2}}=x e^{\sin y}\left(\sin y-\cos ^{2} y\right)$

$\frac{\partial^{2} f}{\partial x^{2}}=e^{\sin x}\left(\cos ^{2} x-\sin x\right)$

$\frac{\partial^{2} f}{\partial y \partial x}=-e^{\sin y} \cos y$.

281) $f=y+y e^{-x}-x=0$

$\frac{\partial f}{\partial y}=1+e^{-x}$

$\frac{\partial f}{\partial x}=-y e^{-x}-1$

$\frac{\partial^{2} f}{\partial y^{2}}=0$

$\frac{\partial^{2} f}{\partial x^{2}}=y e^{-x}$

$\frac{\partial^{2} f}{\partial y \partial x}=-e^{-x}$.

282) $f=\sin x+\sin y+\sin (x-y)=0$

$\frac{\partial f}{\partial y}=\cos y-\cos (x-y)$

$\frac{\partial f}{\partial x}=\cos x+\cos (x-y)$

$\frac{\partial^{2} f}{\partial y^{2}}=-\sin y-\sin (x-y)$

$\frac{\partial^{2} f}{\partial x^{2}}=-\sin x-\sin (x-y)$

$\frac{\partial^{2} f}{\partial y \partial x}=\sin (x-y)$. 
Funktionen von der Form: $x=\varphi(t), y=\psi(t)$. Der erste Differentialquotient ist unter (23), S. 45 , entwickelt:

$$
\frac{d y}{d x}=\frac{d y}{d t}: \frac{d x}{d t} .
$$

Wird auf beiden Seiten nach $x$ differentiiert, so entsteht:

$$
\frac{d^{2} y}{d x^{2}}=\frac{\frac{d^{2} y}{d t^{2}} \cdot \frac{d t}{d x} \cdot \frac{d x}{d t}-\frac{d^{2} x}{d t^{2}} \cdot \frac{d t}{d x} \cdot \frac{d y}{d t}}{\left(\frac{d x}{d t}\right)^{2}}
$$

Unter Berücksichtigung der Relation $\frac{d t}{d x}=1: \frac{d x}{d t}$ geht diese Formel uber in

$$
\frac{d^{2} y}{d x^{2}}=\frac{\frac{d^{2} y}{d t^{2}} \cdot \frac{d x}{d t}-\frac{d^{2} x}{d t^{2}} \cdot \frac{d y}{d t}}{\left(\frac{d x}{d t}\right)^{3}}
$$

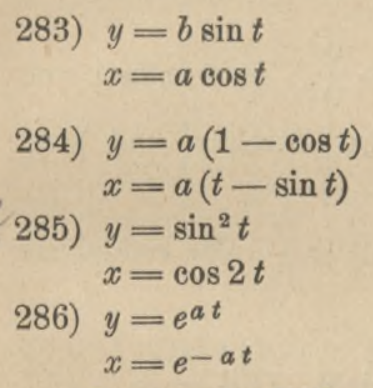

A ufgaben.

Der zweite Differentialquotient kann aus dem ersten auch auf folgende Weise abgeleitet werden:

$$
\frac{d^{2} y}{d x^{2}}=\frac{d\left(\frac{d y}{d x}\right)}{d t} \cdot \frac{d t}{a x}
$$

Beispiel: $y=\frac{1}{1-t}, x=\lg t$;

$$
\frac{d y}{d x}=\frac{t}{(1-t)^{2}}, \quad \frac{d\left(\frac{d y}{d x}\right)}{d t}=\frac{1+t}{(1-t)^{3}}, \frac{d t}{d x}=t, \frac{d^{2} y}{d x^{2}}=\frac{t^{2}+t}{(1-t)^{3}} .
$$


\& 9. Anwendung der Differentialrechnung zur Ermittelung der Werte unbestimmter Formen.

Die Formen $\frac{0}{0}$ und $\frac{\infty}{\infty}$. Es kann sein, dass die beiden Funktionen $\varphi(x)$ und $\psi(x)$ für den nämlichen Wert $x=a$ verschwinden. Ein Bruch von der Form $f(x)=\frac{\varphi(x)}{\psi(x)}$ hat dann für $x=a$ den unbestimmten Wert $f(a)=\frac{0}{0}$. Dass aber trotz dieser Unbestimmtheit der Wert ein bestimmter sein kann, soll zunächst an Beispielen erläutert werden.

1. Beispiel: $f(x)=\frac{x^{3}-a^{3}}{x^{2}-a^{2}}, f(a)=\frac{0}{0}$. Werden aber vor der Substitution $x=a$ Zähler und Nenner durch $(x-a)$ dividiert, so wird $f(x)=\frac{x^{2}+a x+a^{2}}{x+a}$, woraus dann $f(a)=\frac{3}{2} a$ gefunden wird.

2. Beispiel: $f(x)=\frac{e^{x}-1}{x}, f(0)=\frac{0}{0}$. Zieht man 1 von der Reihe für $e^{x}$ ab, dividiert den Rest durch $x$ und setzt dann $x=0$, so findet $\operatorname{man} f(0)=1$.

3. Beispiel: $f(x)=\frac{1-\cos x}{\sin ^{2} x} ; f(0)=\frac{0}{0}$. Die reducierte Form: $f(x)=\frac{2 \sin ^{2} \frac{x}{2}}{4 \sin ^{2} \frac{x}{2} \cos ^{2} \frac{x}{2}}=\frac{1}{2 \cos ^{2} \frac{x}{2}}$ giebt: $f(0)=\frac{1}{2}$.

4. Beispiel: $f(x)=\frac{x^{2}-\sqrt{a^{3} x}}{\sqrt{a x}-a} ; f(a)=\frac{0}{0}$. $f(a)=\left(\frac{\sqrt{x}}{\sqrt{a}} \cdot \frac{\sqrt{x^{8}}-\sqrt{a^{3}}}{\sqrt{x}-\sqrt{a}}\right)_{x=a}=(x+\sqrt{a x}+a)_{x=a}=3 a$.

Als Ursache der Unbestimmtheit zeigen uns die Beispiele den Umstand an, dass Zähler und Nenner einen gemeinsamen Faktor besitzen, der für $x=a$ verschwindet und so zugleich 
Zähler und Nenner auf Null bringt. Statt nun, wie geschehen, diesen Faktor aufzusuchen und vor der Substitution durch Division zu entfernen, bedienen wir uns mit Vorteil der Methode des Differentiierens. Ist

$$
f(x)=\frac{\varphi(x)}{\psi(x)} \text { und } f(a)=\frac{\varphi(a)}{\psi(a)}=\frac{0}{0}
$$

so ist $f(a+\delta)=\left[\frac{\varphi(x+\delta)}{\psi(x+\delta)}\right]_{x=a}$ nicht mehr unbestimmt und geht in $f(a)$ über, wenn $\delta$ die Grenze 0 erreicht. Da weiter $\varphi(a)=0$ und $\psi(a)=0$ sind, so kann man auch setzen:

$$
f(a+\delta)=\left[\frac{\varphi(x+\delta)-\varphi(x)}{\phi(x+\delta)-\psi(x)}\right]_{x=a}=\left[\frac{\frac{\varphi(x+\delta)-\varphi(x)}{(x+\delta)-x}}{\frac{\psi(x+\delta)-\psi(x)}{(x+\delta)-x}}\right]_{x=a}
$$

Lassen wir $\delta$ in Null übergehen, so wird gleichzeitig:

$\lim f(a+\delta)=f(a)$,

$\lim \left[\frac{\varphi(x+\delta)-\varphi(x)}{(x+\delta)-x}\right]_{x=a}=\left[\varphi^{\prime}(x)\right]_{x=a}=\varphi^{\prime}(a)$,

$\lim \left[\frac{\psi(x+\delta)-\psi(x)}{(x+\delta)-x}\right]_{x=a}=\left[\psi^{\prime}(x)\right]_{x=a}=\psi^{\prime}(a)$,

$$
f(a)=\frac{\varphi^{\prime}(a)}{\psi^{\prime}(a)} \text {. }
$$

1. Beispiel; $f(x)=\frac{\varphi(x)}{\psi(x)}=\frac{x^{2}-5 x+6}{x^{3}-2 x^{2}-x+2}, f(2)=\frac{0}{0}$, $\varphi^{\prime}(x)=2 x-5, \psi^{\prime}(x)=3 x^{2}-4 x-1, f(2)=\frac{\varphi^{\prime}(2)}{\psi^{\prime}(2)}=-\frac{1}{3}$. 2. Beispiel: $f(x)=\frac{\sin x}{x} ; f(0)=\left(\frac{\cos x}{1}\right)_{x=0}=1$.

3. Beispiel: $f(x)=\frac{a^{x}-1}{x a^{x}} ; \quad f(0)=\frac{0}{0}, \quad \varphi^{\prime}(0)=\lg a$, $\psi^{\prime}(0)=\left(a^{x}+x a^{x} \lg a\right)_{x=0}=1, f(0)=\lg a$.

Es kommt vor, dass ein Bruchwert auch in der neuen Form noch unbestimmt bleibt, indem $\varphi^{\prime}(a)=0$ und $\psi^{\prime}(a)=0$ 
ist. Das angezeigte Verfahren der Wertermittelung muss dann bei der neuen Form unverändert wiederholt werden.

4. Beispiel: $f(x)=\frac{x^{3}-a x^{2}-a^{2} x+a^{3}}{2 x^{3}-3 a x^{2}+a^{3}} ; f(a)=\frac{0}{0}$, daher $f(a)=\left[\frac{3 x^{2}-2 a x-a^{2}}{6 x^{2}-6 a x}\right]_{x=a}=\frac{0}{0}$ und $f(a)=\left[\frac{6 x-2 a}{12 x-6 a}\right]_{x=a}=\frac{2}{3}$.

5. Beispiel: $f(x)=\frac{\sin 2 x-2 \sin x}{2 e^{x}-x^{2}-2 x-2} ; f(0)=\frac{0}{0}$. Die Unbestimmtheit verschwindet erst nach dreimaliger Differentiation von Zähler und Nenner, und nan findet:

$$
f(0)=\left[\frac{\varphi^{\prime \prime \prime}(x)}{\psi^{\prime \prime \prime}(x)}\right]_{x=0}=\left[\frac{2 \cos x-8 \cos 2 x}{2 e^{x}}\right]_{x=0}=-3 .
$$

Will man von der Voraussetzung ausgehen, dass $\varphi(x)$ und $\phi(x)$ nur darum für $x=a$ gleichzeitig verschwinden, weil sie den gemeinsamen Faktor $(x-a)$ besitzen, so kann (25) etwas kurzer auf folgende Weise entwickelt werden:

$$
f(x)=\frac{\varphi(x)}{\psi(x)}=\frac{(x-a) \varrho(x)}{(x-a) \pi(x)}, f(a)=\frac{\varrho(a)}{\pi(a)} ;
$$

$$
\varphi^{\prime}(x)=\varrho(x)+(x-a) \varrho^{\prime}(x), \quad \psi^{\prime}(x)=\pi(x)+(x-a) \pi^{\prime}(x) \text {. }
$$

Für $x=a$, aber auch nur für diesen Wert von $x$, wird $\varrho(a)=\varphi^{\prime}(a), \quad \pi(a)=\psi^{\prime}(a)$ und $f(a)=\frac{\varphi^{\prime}(a)}{\psi^{\prime}(a)}$.

Auch eine geometrische Ableitung der Formel (25) ist möglich. Wenn $A B$ die Funktionskurve des Zählers $u=\varphi(x)$, $A C$ diejenige des Nenners $v=\psi(x)$ und $\varphi(a)=0, \psi(a)=0$ ist, so müssen sich die beiden Kurven in dem Punkt $A$ schneiden, da ihm die Werte $x=a, u=0, v=0$ entsprechen. Nun sollen dem beliebigen Werte $x=O P$ die Funktionswerte $u=\varphi(x)=B P$ und $v=\psi(x)=C P$ zugehören. Es ist dann

$$
f(x)=\frac{u}{v}=\frac{(x-a) \operatorname{tg} \alpha}{(x-a) \operatorname{tg} \beta}=\frac{\operatorname{tg} \alpha}{\operatorname{tg} \beta} .
$$

Geht $x$ in $a$ über, so werden die Sekanten $A B$ und $A C$ zu Tangenten der entsprechenden Kurven im Punkt $A$, und damit 
Fig. 4.

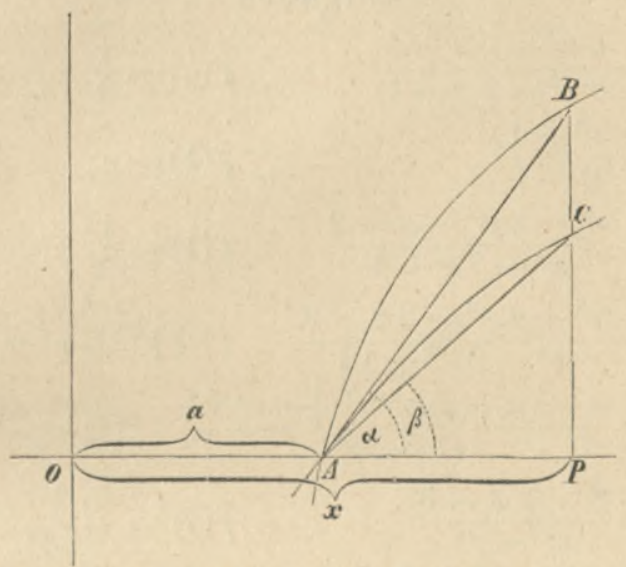

geht zugleich $\operatorname{tg} \alpha$ in $\varphi^{\prime}(a), \operatorname{tg} \beta$ in $\psi^{\prime}(a)$ tiber. Für $x=a$ ist mithin

$$
\begin{gathered}
f(a)=\left(\frac{u}{v}\right)_{x=a}=\frac{\varphi^{\prime}(a)}{\psi^{\prime}(a)} . \\
\text { Wird } f(a)=\left[\frac{\varphi(x)}{\psi(x)}\right]_{x=a}=\frac{\infty}{\infty}, \text { so ist } f(a)=\left[\frac{\frac{1}{\psi(x)}}{\frac{1}{\varphi(x)}}\right]_{x=a}=\frac{0}{0}
\end{gathered}
$$
und damit ist diese Form auf die vorhergehende zurtiokgefuhrt. Wir finden so:

$$
\begin{gathered}
f(a)=\left[\frac{\frac{-\psi^{\prime}(x)}{[\psi(x)]^{2}}}{\frac{\varphi^{\prime}(x)}{[\varphi(x)]^{2}}}\right]_{x=a}=\left[\frac{\phi^{\prime}(x)}{\varphi^{\prime}(x)} \cdot\left(\frac{\varphi(x)}{\psi(x)}\right)^{2}\right]_{x=a} \\
f(a)=\frac{\psi^{\prime}(a)}{\varphi^{\prime}(a)} \cdot[f(a)]^{2}, \quad f(a)=\frac{\varphi^{\prime}(a)}{\psi^{\prime}(a)}
\end{gathered}
$$

Hat ein Bruch für $x=a$ die unbestimmte Form $\frac{0}{0}$ oder $\frac{\infty}{\infty}$, so findet man seinen Wert, indem man Zähler und Nenner differentiiert und dann erst $x=a$ setzt. 


\section{Aufgaben.}

287) $f(x)=\frac{6 x^{2}-5 x+1}{8 x^{2}-2 x-1}$

$f\left(\frac{1}{2}\right)=\frac{1}{6}$

288) $\quad n=\frac{x^{n}-1}{x-1}$

$f(1)=n$

289) $n=\frac{x^{3}-2 x^{2}-x+2}{x^{3}-7 x+6}$

$f(1)=\frac{1}{2}, f(2)=\frac{3}{5}$

290) $"=\frac{x^{3}+4 x^{2}-2 x-8}{x^{4}-8 x^{2}+12}$

$f( \pm \sqrt{2})=\frac{-4 \mp \sqrt{2}}{4}$

291) $"=\frac{x-(n+1) x^{n+1}+n x^{n+2}}{(1-x)^{2}}$

$f(1)=\frac{n(n+1)}{2}$

292) $"=\frac{2-\sqrt{x-3}}{x^{2}-49}$

$f(7)=-\frac{1}{56}$

293) $n=\frac{\sqrt{3 x}-\sqrt{12-x}}{2 x-3 \sqrt{19-5 x}}$

$f(3)=\frac{8}{69}$

294) $n=\frac{a-\sqrt[5]{2 x^{5}-a^{5}}}{\sqrt[3]{x^{8}-a^{3}}}$

$f(a)=0$

295) $"=\frac{\sqrt{2 a^{3} x-x^{4}}-a \sqrt[3]{a^{2} x}}{a-\sqrt[4]{a x^{3}}} f(a)=\frac{16}{9} a$

296) $\quad n=\frac{x}{e^{x}-e^{-x}}$

$f(0)=\frac{1}{2}$

297) $\quad " \quad=\frac{e^{x}-e^{-x}}{1-x-\lg (e-x)}$

$f(0)=\frac{2 e}{1-e}$

298) $\quad "=\frac{x e^{\cos x}}{1-\sin x-\cos x}$

$f(0)=-e$

299) $"=\frac{e^{x}-e^{\sin x}}{x-\sin x}$

$f(0)=1$

300) $"=\frac{a^{x}-1}{x}$

$f(0)=\lg a$

301) $"=\frac{\lg x}{\sqrt{x^{2}-1}}$

$f(1)=0$

302) $"=\frac{a^{\lg x}-x}{\lg x}$

$f(1)=\lg a-1$ 
303) $f(x)=\frac{\lg \left(x^{2}-3\right)}{x^{2}+3 x-10}$

304) $\quad=\frac{\lg (a+x)-\lg a}{x}$

305) $\quad=\frac{\lg \frac{x+\sqrt{x^{2}-a^{2}}}{a}}{\sqrt{x^{2}-a^{2}}}$

306) $\quad n=\frac{\sin (n x)}{x}$

307) $"=\frac{\sin x}{\sin 2 x}$

308). $\quad "=\frac{\sin (a x)}{\sin (b x)}$

309) $\quad n=\frac{2 \sin x-1}{\cos 3 x}$

310) $"=\frac{x \cos x}{x-\sin x}$

311) $\quad=\frac{x \cos x-\sin x}{x^{2}}$

312) $n=\frac{\sin 2 x-2 \sin x}{2 e^{x}-2-2 x}$

313) $"=\frac{2 \operatorname{tg}^{2} x-\operatorname{cotg} x-1}{2 \sin ^{2} x-\cos ^{2} x-\frac{1}{2}}$

314),$=\frac{\operatorname{tg}^{2} x-2 \operatorname{tg} x-3}{\operatorname{tg}^{2} x-4 \operatorname{tg} x+3}$

315) $"=\frac{\arcsin (2-x)}{\sqrt{x^{2}-3 x+2}}$

316) $\quad=\frac{\arcsin \frac{\sqrt{a^{2}-x^{2}}}{a}}{\sqrt{a^{2}-x^{2}}}$

317) $"=\frac{\arccos (1-x)}{\sqrt{2 x-x^{2}}}$

318),$=\frac{\lg \cos x}{x}$ $f(2)=\frac{4}{7}$
$f(0)=\frac{1}{a}$

$f(a)=\frac{1}{a}$

$f(0)=n$

$f(\pi)=-\frac{1}{2}$

$f(0)=\frac{a}{b}$

$f\left(\frac{\pi}{6}\right)=-\frac{1}{3} \sqrt{3}$

$f(0)=\frac{1}{0}$

$f(0)=0$

$f(0)=0$

$f\left(\frac{\pi}{4}\right)=\frac{10}{3}$

$f(\operatorname{arctg} 3)=2$

$f(2)=0$

$f(a)=\frac{1}{a}$

$f(0)=1$

$f(0)=0$ 
319) $f(x)=\frac{\sin x-\cos x}{1-\operatorname{tg} x}$

320) $\pi=\frac{\lg \left(1+e^{x}\right)}{a+b x}$

321) $n=\frac{\lg (a+x)}{x}$

322) $\quad "=\frac{x^{4}}{e^{x}}$

323) $\quad=\frac{\operatorname{tg} x}{\operatorname{tg} 3 x}$

324) $\quad=\frac{\operatorname{cotg} 2 x}{\operatorname{cotg} x}$

325) $\quad=\frac{\frac{\pi}{x}}{\operatorname{cotg} \frac{x \pi}{2}}$

$$
\begin{aligned}
& f\left(\frac{\pi}{4}\right)=-\sqrt{\frac{1}{2}} \\
& f(\infty)=\frac{1}{b} \\
& f(\infty)=0
\end{aligned}
$$$$
f(\infty)=0
$$$$
f\left(\frac{\pi}{2}\right)=3
$$$$
f(0)=\frac{1}{2}
$$

$$
f(0)=\frac{\pi^{2}}{2}
$$

Die Form $\infty-\infty$. Wenn in $f(x)=\frac{\varphi(x)}{\psi(x)}-\frac{\pi(x)}{\varrho(x)}$ nur $\phi(a)$ und $\varrho(a)$ gleich Null werden, so ist $f(a)=\infty-\infty$. Man setzt dann vor der Substitution $x=a$ statt der Differenz den Quotienten $[\varphi(x) \varrho(x)-\pi(x) \psi(x)]: \psi(x) \varrho(x)$, welcher für $x=a$ die unbestimmte Form $\frac{0}{0}$ annimmt.

326) $f(x)=\frac{1}{x^{5}}-\frac{1}{\sin x}=\frac{\sin x-x^{8}}{x^{3} \sin x} \quad f(0)=\infty$

327) $"=\frac{x}{\operatorname{cotg} x}-\frac{\frac{\pi}{2}}{\cos x} \quad f\left(\frac{\pi}{2}\right)=-1$

328) $\quad \pi=\frac{1}{x-1}-\frac{1}{\lg x}$ $f(1)=-\frac{1}{2}$

329) $\quad n=\frac{1}{\sin x}+\frac{1}{\lg (1-x)}$ $f(0)=+\frac{1}{2}$

330) $\quad=\operatorname{tg} x-\frac{1}{1-\sin x}$ $f\left(\frac{\pi}{2}\right)=\infty$ 
Die Form $0 . \infty$ entsteht, wenn in $f(x)=\varphi(x) \cdot \psi(x)$ für $x=a$ der eine Faktor verschwindet, während der zweite unendlich gross wird. Man giebt dann der Funktion die Form:

$$
f(a)=\left[\frac{\varphi(x)}{\frac{1}{\phi(x)}}\right]_{x=a}=\frac{0}{0} \text { oder }=\frac{\infty}{\infty},
$$

je nachdem $\varphi(a)=0, \psi(a)=\infty$ ist, oder umgekehrt.

331) $f(x)=\frac{1}{e^{x}-1} \cdot \lg a^{x}$

$$
f(0)=\lg a
$$

332) $"=x \cdot \lg \left(1+\frac{1}{x}\right)$

$$
f(\infty)=1
$$

333) $\quad n=(1-x) \operatorname{tg} \frac{\pi}{2} x$

$f(1)=\frac{2}{\pi}$

334) $\cap=\arcsin \frac{x-a}{a} \cdot \operatorname{cotg}(x-a) f(a)=\frac{1}{a}$

335) $\quad \pi=(1-\sin x) \cdot \operatorname{tg} x$

$$
f\left(\frac{\pi}{2}\right)=0 \text {. }
$$

Weitere unbestimmte Formen. Die Funktion $y=u^{v}$, worin $u$ und $v$ Funktionen von $x$ sind, lässt sich auch so schreiben $y=e^{v \lg u}$, weil $\lg y=v \lg u$. Wird nun für $x=a$

1) $u=0$ und $v=0$, so ist $y=0^{0}$,

2) $u=\infty \quad n \quad v=0, \quad n y=\infty^{0}$,

3) $u=1 \quad, v=\infty, \quad n y=1^{\infty}$.

Wenn endlich $y=\sqrt[v]{u}$ ist, so ist

$\lg y=\frac{1}{v} \lg u$, oder $y=e^{\frac{1}{v} \lg u}$. Wird dann für $x=a$
4) $u=1$ und $v=0$, so ist $y=\sqrt[0]{1}$,
5) $u=0 \quad, \quad v=\infty, \quad, \quad, y=\sqrt[\infty]{0}$,
6) $u=\infty \quad, v=\infty, \quad, y=\sqrt[\infty]{\infty}$.

In diesen Fällen wird der Exponent von $e$ gleich $0 . \infty$.

336) $f(x)=x^{x}$

$f(0)=1$

337) $n=x^{\frac{1}{x}}$

$f(\infty)=1$ 
338) $f(x)=(\sin x)^{\frac{1}{\cos x}}$

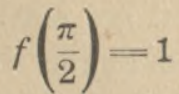

339) $n=(\sin x)^{\operatorname{tg} x}$ $f\left(\frac{\pi}{2}\right)=1$.

Anmerkung. Wenn, wie es vorkommt, die unbestimmte Form irgend eines Ausdrucks durch Differentation nicht beseitigt werden kann, so nimmt man in der Regel dazu die Reihenentwicklung zu Hilfe.

§ 10. Maxima und Minima der Funktionen.

Entwickelte Funktionen. Wir setzen voraus, dass die Funktion $y=f(x)$ von $x=x_{0}$ bis $x=x_{0}+h$ endlich und stetig variabel sei (S. 7). Man kann für die Stellen $x$ dieses Gebietes die Formel (2), S. 7, so schreiben:

$$
f(x+\Delta x)-f(x)=\Delta x\left[f^{\prime}(x)+\varepsilon\right]
$$

wobei $\varepsilon$ eine veränderliche Grösse bedeutet, welche mit $\Delta x$ gleichzeitig unendlich klein ist. Nun nehmen wir an, $f^{\prime}(x)$ sei von Null verschieden. Dann kann man $\Delta x$ so klein wählen, dass $|\varepsilon|<\left|f^{\prime}(x)\right|$ wird (vgl. S. 6); dadurch erhält die eckige Klammer auf der rechten Seite der letzten Gleichung: das Vorzeichen von $f^{\prime}(x)$. Da die linke Seite den Zuwachs der Funktion $f(x)$ darstellt, so können wir sagen:

Ist der Differentialquotient $f^{\prime}(x)$ von Null versohieden, dann kann man um $x$ ein kleines Gebiet derart abgrenzen, dass in ihm bei positivem $f^{\prime}(x)$ die Zunahmen der Variabelen und der Funktion dasselbe Zeichen haben, während sie bei negativem $f^{\prime}(x)$ entgegengesetzte Zeichen besitzen. Mit anderen Worten: Bei positivem $f^{\prime}(x)$ wächst die Funktion gleichzeitig: mit der Variabelen und nimmt gleichzeitig mit ihr ab. Bei negativem $f^{\prime}(x)$ wächst die Funktion, wenn die Variabele abnimmt und umgekehrt.

Zur Ergänzung dieser Sätze nehmen wir jetzt an, $f^{\prime}(x)$ sei für eine beliebig kleine aber endliche Strecke gleich Null, 
etwa zwischen $a$ und $b$, wobei $b>a$ sein mag. Dann setzen wir mit Benutzung einér willkürlichen reellen Konstanten $x$

$$
\Phi(x)=f(x)-x^{2} x, \quad \varphi(x)=f(x)+x^{2} x
$$

und finden durch Differentiation

$$
\Phi^{\prime}(x)=f^{\prime}(x)-x^{2}=-x^{2}, \quad \varphi^{\prime}(x)=f^{\prime}(x)+x^{2}=+x^{2} .
$$

Nach dem oben abgeleiteten Satze wächst also $\varphi(x)$ beständig auf der Strecke von $a$ bis $b$, während $\Phi(x)$ auf ihr beständig abnimmt. Demnach ist $\Phi(b)<\Phi(a)$ und $\varphi(b)>\varphi(a)$, d. h.

$$
f(b)-f(a)<x^{2}(b-a) \text { und } f(b)-f(a)>-x^{2}(b-a) \text {. }
$$

Wäre nun $f(b)$ von $f(a)$ verschieden, so würde hieraus ein Widerspruch folgen, da man $x^{2}$ so klein wählen könnte, dass

$$
|f(b)-f(a)|>|b-a| x^{2}
$$

wäre. Es muss demnach $f(b)=f(a)$ sein; und da die gleichen Schlüsse für jeden zwischen $a$ und $b$ gelegenen Punkt gelten, so baben wir bewiesen: Ist für eine endliche Strecke der Differentialquotient $f^{\prime}(x)=0$, so ist für diese Strecke die Funktion $f(x)$ konstant.

Die abgeleiten Sätze lassen sich umkehren: Wächst die Funktion $f(x)$, so ist der Differentialquotient $f^{\prime}(x)$ positiv oder auch für einzelne Punkte Null; nimmt die Funktion $f(x) \mathbf{a b}$, so ist der Differentialquotient negativ oder auch für einzelne Punkte Null. Ist die Funktion $f(x)$ konstant, dann hat der Differentialquotient $f^{\prime}(x)$ den Wert Null und umgekehrt.

Die eben entwickelten Sätze lassen sich sehr einfach geometrisch veranschaulichen, wenn $\operatorname{man} x$ und $y$ als die rechtwinkligen Koordinaten einer ebenen Kurve ansieht. Dann ist $\frac{d y}{d x}=f^{\prime}(x)$ die trigonometrische Tangente des Winkels, den die im Punkte $(x, y)$ angelegte Bertihrungslinie der Kurve mit der Richtung der wachsenden $x$ einschliesst ( $\S 2$, S. 5 ), und zwar soll die Bertihrungslinie immer in der Richtung vom Punkte $(x, y)$ nach dem Punkte $(x+d x, y+d y)$ genommen werden. Ist nun die Funktion $y=f(x)$ für das wachsende $x$ im Zunehmen begriffen, so steigt die Kurve, wenn man sie 
im Sinne der wachsenden $x$ durchläuft. Es sind $d x$ und $d y$ beide positiv. (Fig. 5a an der Stelle M, Fig. 6a an der Stelle $M_{m}$, Fig. $7 \mathrm{a}$ an den Stellen $M$, und $M_{m}$.) Die Berührungslinie schliesst mit der Richtung der wachsenden $x$ einen Winkel ein, dessen Kosinus und Sinus beide positiv sind, nämlich der Kosinus $=\frac{d x}{\sqrt{d x^{2}+d y^{2}}}$ und der Sinus $=\frac{d y}{\sqrt{d x^{2}+d y^{2}}} \cdot$ (Es ist dies der positive spitze Winkel $\alpha$, in Fig. $5 \mathrm{a}$, der positive spitze Winkel $\alpha_{\prime \prime}$ in Fig. $6 \mathrm{a}$, und in Fig. $7 \mathrm{a}$ sind es resp. die positiven spitzen Winkel $\alpha$, und $\alpha_{\prime \prime}$.) Ist dagegen die Funktion $y=f(x)$ für wachsende $x$ im Abnehmen begriffen, so fällt die Kurve, wenn man sie im Sinne der wachsenden $x$ durchläuft. Hier ist $d x$ positiv und $d y$ negativ. (Fig. 5a an der Stelle $M_{n}$, Fig. $6 \mathrm{a}$ an der Stelle $M_{1}$, Fig. $8 \mathrm{a}$ an den Stellen $M$, und $M_{n}$.) Die Berührungslinie in der oben angegebenen Richtung schliesst mit der Richtung der wachsenden $x$ einen Winkel ein, dessen Kosinus positiv, dessen Sinus negativ ist. (Es ist dies der negative spitze Nebenwinkel von $\alpha_{\prime \prime}$ in Fig. 5a, von $\alpha$, in Fig. 6a, von $\alpha$, und von $\alpha_{n}$, in Fig. 8a.) Diese Winkel liegen unterhalb der Abseissenaxe und werden hergestellt, indem man die Bertihrungslinie tiber ihren Schnittpunkt mit der Abscissenaxe hinaus verlängert.

Hiernach kann man die oben entwickelten Sätze auch so aussprechen:

Wenn die Kurve steigt, so schliesst ihre Berührungslinie in der Richtung vom Punkte $(x, y)$ nach dem Punkte $(x+d x$, $y+d y)$ mit der Richtung der wachsenden $x$ einen positiven spitzen Winkel ein, und umgekehrt.

Wenn die Kurve fällt, so schliesst ihre Berührungslinie in der Richtung vom Punkte $(x, y)$ nach dem Punkte $(x+d x$, $y+d y)$ mit der Richtung der wachsenden $x$ einen negativen spitzen Winkel ein, und umgekehrt. 
Fig. 5 a.

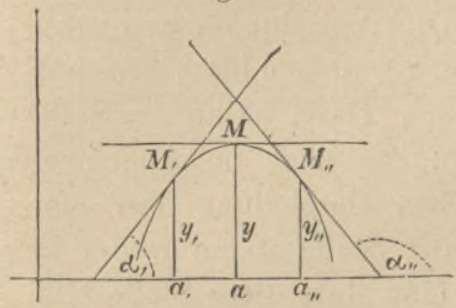

Fig. 5 b.

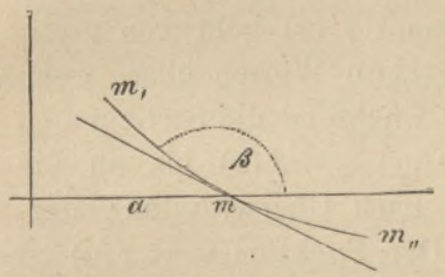

Fig. 7 a.

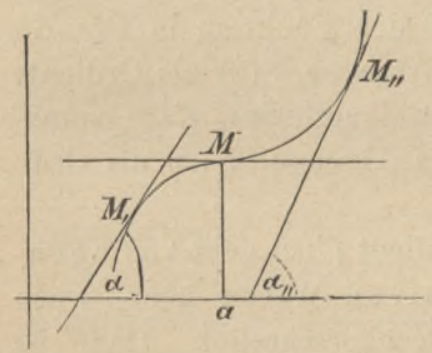

Fig. 7 b.

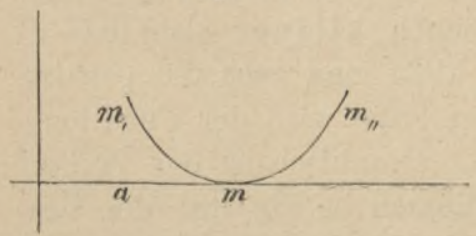

Fig. 6 a.

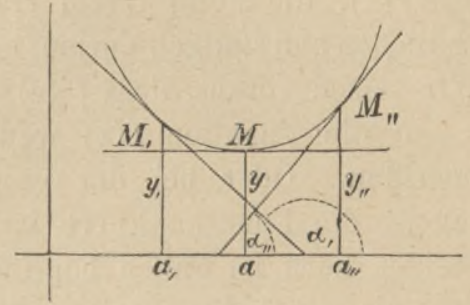

Fig. $6 \mathrm{~b}$.

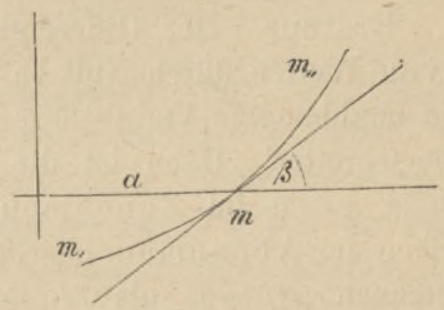

Fig. 8 a.

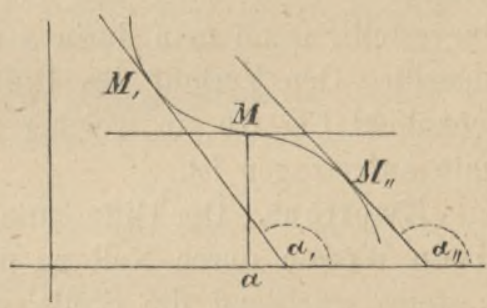

Fig. 8 b.

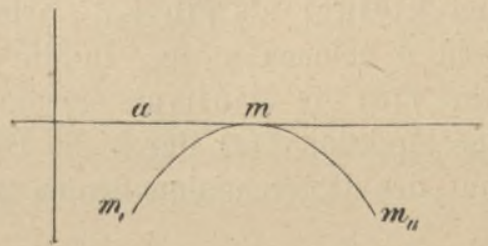


Nach dieser Vorbereitung betrachten wir eine Funktion $y=f(x)$, die nebst ihrem ersten Differentialquotienten einen endlichen und stetigen Verlauf hat von $x=a-\delta$ bis $x=a+\delta$. Wir setzen voraus, dass $f^{\prime}(a)=0$ sei. Dann ist an der Stelle $x=a$ die Funktion $f(x)$ weder im Zu- noch im Abnehmen begriffen. Oder bei der graphischen Darstellung darf man sagen: die Funktionskurve ist an der Stelle $x=a$ weder im Steigen noch im Fallen begriffen. Man darf diese Stelle bildlich als ein momentanes Niveau bezeichnen. Hier sind vier Fälle zu untersoheiden:

Erstens. Der Differentialquotient $f^{\prime}(x)$ geht von positiven Werten durch Null zu negativen Werten tber, wenn die unabhängige Variabele $x$ stetig wachsend die Stelle $x=a$ ubersohreitet. Dann ist die Funktion $y=f(x)$ bis an die Stelle $x=a$ im Zunehmen und nach Überschreitung derselben im Abnehmen begriffen. Der Funktionswert $f(a)$ ist demnach grösser als $f(a-\delta)$ und grösser als $f(a+\delta)$, wie klein man auch die positive Zahl $\delta$ nehmen möge. In diesem Falle nennt man den Funktionswert $f(a)$ ein Maximum. Graphisch findet sich der Verlauf der Funktion in Fig. 5 a dargestellt, wenn man darin $x$ als Abscisse, $f(x)$ als Ordinate ansieht. Den Verlauf des Differentialquotienten $f^{\prime}(x)$ veranschaulicht Fig. 5 b, in welcher $x$ als Abscisse, $f^{\prime}(x)$ als Ordinate aufgetragen ist.

Zweitens. Der Differentialquotient $f^{\prime}(x)$ geht von negativen Werten durch Null zu positiven Werten über, wenn $x$ stetig wachsend die Stelle $x=a$ überschreitet. Dann ist die Funktion $f(x)$ bis an die Stelle $x=a$ im Abnehmen und nach Überschreitung derselben im Zunehmen begriffen. Der Funktionswert $f(a)$ ist demnach kleiner als $f(a-\delta)$ und kleiner als $f(a+\delta)$, wie klein man auch die positive Zahl $\delta$ nehmen möge. In diesem Falle wird der Funktionswert $f(a)$ ein Minimum genannt. Graphisch ist der Verlauf der Funktion $f(x)$ durch die Ordinaten in Fig. 6a, der Verlauf des Differentialquotienten $f^{\prime}(x)$ duroh die Ordinaten in 
Fig. $6 \mathrm{~b}$ veranschaulicht. Die unabhängige Variabele $x$ ist in beiden Figuren als Abscisse genommen.

Drittens. Der Differentialquotient $f^{\prime}(x)$ ist positiv für $x=a-\delta$, erreicht stetig abnehmend den Wert Null für $x=a$ und nimmt nach Überschreitung dieser Stelle stetig $\mathrm{zu}$, so dass er fuir $x=a+\delta$ wieder positiv ist, wie klein auch $\delta$ genommen werden möge. Dann ist die Funktion $f(x)$ vor und nach der Stelle $x=a$ im Zunehmen begriffen. Sie hat also an dieser Stelle weder Maximum noch Minimum. Die Funktion $f(x)$ wird durch die Ordinaten in Fig. 7a, ihr Differentialquotient $f^{\prime}(x)$ durch die Ordinaten in Fig. 7b dargestellt.

Viertens. Der Differentialquotient $f^{\prime}(x)$ ist negativ für $x=a-\delta$, erreicht stetig wachsend den Wert Null für $x=a$ und nimmt nach Überschreitung dieser Stelle stetig $\mathrm{ab}$, so dass er für $x=a+\delta$ wieder negativ ist, wie klein auch $\delta$ genommen werden möge. Dann ist die Funktion $f(x)$ vor und nach der Stelle $x=a$ im Abnehmen begriffen. Sie hat also an dieser Stelle weder Maximum nooh Minimum. Ihr Verlauf wird durch die Ordinaten in Fig. 8a, der Verlauf des Differentialquotienten $f^{\prime}(x)$ durch die Ordinaten in Fig. 8 b vor Augen geführt.

[Wenn eine ebene Kurve in einem gewissen Punkte von einer geraden Linie zugleich berührt und geschnitten wird, so nennt man diesen Punkt einen Wendepunkt. In dem dritten und vierten der eben untersehiedenen Fälle hat die Funktionskurve einen Wendepunkt, und ihre Tangente in diesem Punkte liegt hier speciell parallel zur Abseissenachse.]

Man kann die gewonnenen Resultate auch so zusammenfassen:

I. Eine Funktion $y=f(x)$, die nebst ihrem ersten Differentialquotienten einen endlichen und stetigen Verlauf hat von $x=a-\delta$ bis $x=a+\delta$, besitzt an der Stelle $x=a$ ein Maximum (Minimum), wenn der Differentialquotient $f^{\prime}(x)$ an dieser Stelle dureh Null 
hindurchgehend sein Vorzeichen ändert. Ein Maximum findet statt, wenn $f^{\prime}(a-\delta)>0$ und $f^{\prime}(a+\delta)<0$; ein Minimum dagegen, wenn $f^{\prime}(a-\delta)<0$ und $f^{\prime}(a+\delta)>0$ sind.

II. Wenn aber der Differentialquotient $f^{\prime}(x)$ mit wachsendem $x$ an der Stelle $x=a$ stetig variabel durch Null hindurchgeht, ohne sein Vorzeichen zu ändern, so hat die Funktion $f(x)$ an dieser Stelle weder Maximum noch Minimum.

Wir setzen jetzt voraus, dass die Funktion $y=f(x)$ und ihre aufeinander folgenden Differentialquotienten $f^{\prime}(x), f^{\prime \prime}(x)$, $f^{\prime \prime \prime}(x), \ldots$ bis zu dem von der $n^{\text {ten }}$ Ordnung: $f^{n}(x)$ von $x=a-\delta$ bis $x=a+\delta$ endlich und stetig variabel verlaufen. Es soll ferner $f^{n}(x)$ der Differentialquotient von der niedrigsten Ordnung sein, welcher für $x=a$ einen von Null verschiedenen Wert hat. Alle vorhergehenden Differentialquotienten sollen für $x=a$ zu Null werden: $f^{\prime}(a)=f^{\prime \prime}(a)=\ldots=f^{n-1}(a)=0$. Dann lässt sich $\delta$ positiv und so klein wählen, dass von $x=a-\delta$ bis $x=a+\delta$ der Differentialquotient $f^{n}(x)$ sein Vorzeichen nicht ändert. Infolgedessen geht $f^{n-1}(x)$ mit wachsendem $x$ an der Stelle $x=a$ von positiven zu negativen Werten tiber oder umgekehrt von negativen zu positiven Werten, je nachdem $f^{n}(a)$ negativ oder positiv ist. Im erstgenannten Falle ist $f^{n-2}(a)$ ein Maximum, im zweiten Falle dagegen ein Minimum, wie aus dem Satze I. ohne weiteres hervorgeht. Jedenfalls sieht man, dass $f^{n-2}(x)$ zwischen $a-\delta$ und $a+\delta$ sein Vorzeichen nicht ändert, und dass dies Vorzeichen dasselbe ist wie bei $f^{n}(x)$. Danach erhält man bei $f^{n-3}(x)$ dieselbe Vorzeichen-Änderung wie bei $f^{n-1}(x)$, und es wiederholen sich periodisch dieselben Erscheinungen, wenn man schrittweise zu Differentialquotienten von geringerer Ordnung tubergeht.

Ist nun $n$ eine gerade Zahl und $f^{n}(a)$ negativ, so gelangt man zu der Einsicht, dass $f^{\prime \prime}(x)$ negativ ist. Folglich geht $f^{\prime}(x)$ mit wachsendem $x$ an der Stelle $x=a$ von posi- 
Fig. 9.
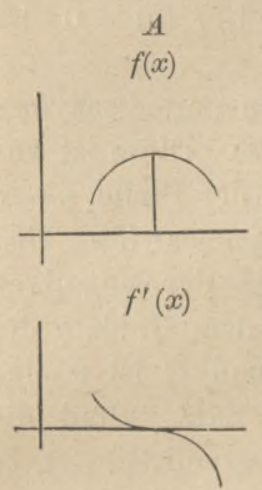

$f^{\prime \prime}(x)$

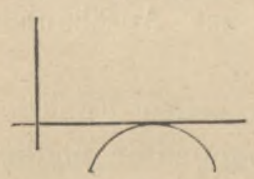

$f^{\prime \prime \prime}(x)$

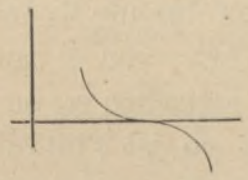

$f^{\prime \prime \prime \prime}(x)$

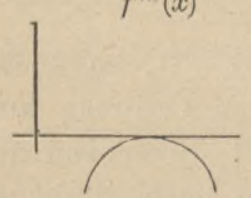

B

$f(x)$

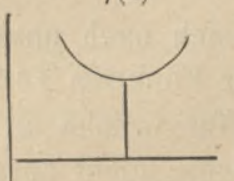

$f^{\prime}(x)$

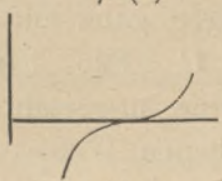

$f^{\prime \prime}(x)$

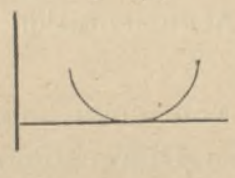

$f^{\prime \prime \prime}(x)$

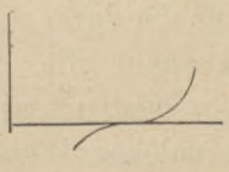

$f^{\prime \prime \prime \prime}(x)$

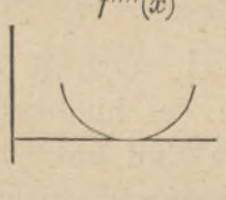

C

$f(x)$

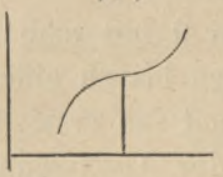

$f^{\prime}(x)$

D

$f(x)$

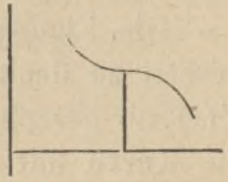

$f^{\prime}(x)$

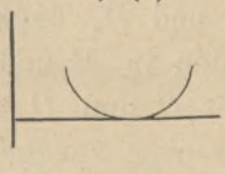

$f^{\prime \prime}(x)$

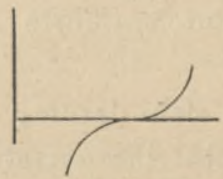

$f^{\prime \prime \prime}(x)$

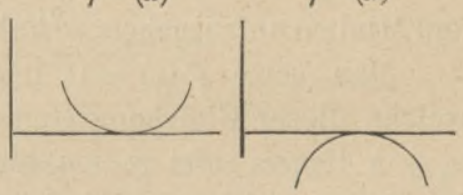

$f^{\prime \prime \prime \prime}(x)$

tiven zu negativen Werten uber, und es ist $f(a)$ ein Maximum. (Fig. 9. A.)

Ist $n$ eine gerade Zahl und $f^{n}(a)$ positiv, so findet sich, dass $f^{\prime \prime}(x)$ positiv ist. Folglich geht $f^{\prime}(x)$ mit wachsenden $x$ an der Stelle $x=a$ von negativen zu positiven Werten über, und es ist $f(a)$ ein Minimum. (Fig. 9 B.)

Ist $n$ ungerade, so hat $f^{\prime}(x)$ positive oder negative Werte, je nachdem $f^{n}(a)$ positiv oder negativ ist. Folglich 
hat $f(x)$ weder Maximum noch Minimum an der Stelle $x=a$, sondern nur ein momentanes Niveau, weil $f^{\prime}(a)=0$ ist. (Fig. 9, $C$ und $D$.)

Die Figuren 9 hat man sich nach unten periodisoh fortgesetzt zu denken bis zu einer Funktion $f^{n}(x)$. Diese ist von $f^{\prime}(x)$ an gerechnet die erste, für welche an der Stelle $x=a$ die Kurve mit der Abscissenachse nicht zusammentrifft. Und zwar verläuft $f^{n}(x)$ ganz auf dem Gebiete der negativen Ordinaten in $A$ und $D$, dagegen ganz auf dem Gebiete der positiven Ordinaten in $B$ und $C$. Für $A$ und $B$ ist $n$ eine gerade Zahl, für $C$ und $D$ eine ungerade Zahl. Sind die Figuren 9 in der eben angegebenen Weise vervollständigt, so hat man sie von unten nach oben sehrittweise zu durchlaufen, um die oben durchgeführte Argumentation zur Anschauung zu bringen.

Handelt es sich darum, von einer gegebenen Funktion $y=f(x)$, die nebst ihren ersten Differentialquotienten endlich und stetig verläuft von $x=x_{0}$ bis $x=x_{0}+h$, die Maxima und Minima aufzusuchen, so haben wir dafur die folgende Regel:

Man setze $f^{\prime}(x)=0$ und suche die Werte von $x$ auf, welche dieser Gleichung Genüge leisten und zwischen $x_{0}$ und $x_{0}+h$ liegen. Ist $x=a$ ein soleher Wert, so ist $f(a)$ ein Maximum oder ein Minimum, je nachdem $f^{\prime \prime}(a)$ negativ oder positiv ist.

Findet sich ausser $f^{\prime}(a)=0$ auch $f^{\prime \prime}(a)=0$, so suche man den Differentialquotienten der niedrigsten Ordnung auf, der für $x=a$ nicht den Wert Null hat. Ist dieser von ungerader Ordnung, so findet weder Maximum noch Minimum statt. Ist er von gerader Ordnung, so ist $f(a)$ ein Maximum oder ein Minimum, je nachdem jener Differentialquotient für $x=a$ einen negativen oder einen positiven Wert hat.

1. Beispiel: Für welche Werte von $x$ wird $f(x)=x+\frac{1}{x}$ ein Max. oderMin. $f^{\prime}(x)=1-\frac{1}{x^{2}}=0$ giebt $x= \pm 1 ; f^{\prime \prime}(x)=\frac{2}{x^{3}}$. 
Da nun $f^{\prime \prime}(1)=2$, d. h. positiv ist, so muss $f(1)=2$ ein Min. sein, sowie $f(-1)=-2$ ein Max. ist, weil $f^{\prime \prime}(-1)=-2$ negativ ist.

2. Beispiel: $f(x)=\sin x+\cos x ; f^{\prime}(x)=\cos x-\sin x=0$, $x=\frac{\pi}{4}$ und $\frac{5 \pi}{4} ; f^{\prime \prime}(x)=-\sin x-\cos x ; f\left(\frac{\pi}{4}\right)=\sqrt{2}$ ist ein Max., weil $f^{\prime \prime}\left(\frac{\pi}{4}\right)<0, f\left(\frac{5 \pi}{4}\right)=-\sqrt{2}$ ist ein Min., weil $f^{\prime \prime}\left(\frac{5 \pi}{4}\right)>0$. Periodische Wiederholung. 3. Beispiel: Fur welchen Wert von $x$ wird $f(x)=\left(\frac{a}{x}\right)^{x}$
ein Max. oder Min.?

$f^{\prime}(x)=\left(\frac{a}{x}\right)^{x}(\lg a-\lg x-1)=0$, oder $\lg x=\lg \frac{a}{e}, x=\frac{a}{e} ;$ $f^{\prime \prime}(x)=-\frac{1}{x} f(x)+f^{\prime}(x)(\lg a-\lg x-1) ; f^{\prime \prime}\left(\frac{a}{e}\right)=-\frac{e}{a} \cdot e^{\frac{a}{e}}$, d. h. negativ, mithin ist $f\left(\frac{a}{e}\right)=e^{\frac{a}{e}}$ ein Max.

4. Beispiel: Für welche Werte von $x$ wird $f(x)=\sin x \sin (\alpha-x)$ ein Max. oder Min.?

$f^{\prime}(x)=\sin (\alpha-2 x)=0$, woraus $\alpha-2 x=0$, oder $=\pi$, oder $=2 \pi$; $x=\frac{\alpha}{2}$, oder $=\frac{\alpha-\pi}{2}$, oder $=\frac{\alpha}{2}-\pi ; f^{\prime \prime}(x)=-2 \cos (\alpha-2 x)$. Nun ist $f^{\prime \prime}\left(\frac{\alpha}{2}\right)=-2$, also $f\left(\frac{\alpha}{2}\right)=\sin ^{2} \frac{\alpha}{2}$ ein Max., $f^{\prime \prime}\left(\frac{\alpha-\pi}{2}\right)=2$, mithin $f\left(\frac{\alpha-\pi}{2}\right)=-\cos ^{2} \frac{\alpha}{2}$ ein Min. Der dritte Wert ist dem ersten gleich. Periodische Wiederholung.

5. Beispiel: Für welche Werte von $x$ wird $f(x)=\frac{\sin x}{1+\operatorname{tg} x}$ ein Max. oder Min.?

$f^{\prime}(x)=\frac{\cos x-\sin x \operatorname{tg}^{2} x}{(1+\operatorname{tg} x)^{2}}=0$, oder $\operatorname{tg}^{3} x=1, x=\frac{\pi}{4}$ und $x=\frac{5 \pi}{4}$. 
Wenn $f^{\prime}(x)=\frac{u}{v}$ ist, so ist $f^{\prime \prime}(x)=\frac{v \cdot u^{\prime}-u \cdot v^{\prime}}{v^{2}}$.

Da nun für den in Betracht kommenden Wert von $x$ der Zähler des Bruches $\frac{u}{v}$ versehwindet, so reduziert sich die letzte Gleichung auf $f^{\prime \prime}(x)=\frac{u^{\prime}}{v}$.

In unserem Beispiele wird $f\left(\frac{\pi}{4}\right)=\frac{1}{4} \sqrt{2}$ ein Max., $f\left(\frac{5 \pi}{4}\right)$ ein Min., weil $f^{\prime \prime}\left(\frac{\pi}{4}\right)$ negativ und $f^{\prime \prime}\left(\frac{5 \pi}{4}\right)$ positiv ist. Periodische
Wiederholung.

\section{Aufgaben.}

$\mathrm{Zu}$ den nachfolgenden Aufgaben sind als Lösungen nur die Werte von $x$ angegeben, für welche die Funktion ihre Maximal- und Minimalwerte annimmt; diese selbst können durch Substitution leicht gebildet werden.

340) $f(x)=x^{3}-2 a x^{2}+a^{2} x \quad$ Mx. für $x=\frac{a}{3}$, Mn.für $x=a$

341) ${ }_{n}=x^{3}+6 x^{2}-15 x \quad$ Mx. , $x=-5$, Mn. für $x=1$

342) $\quad n=x^{2}(1-x)$

Mx. , $x=\frac{2}{3}$, Mn.für $x=0$

343) $"=x^{2}(a-x)^{2}$

Mx.,$x=\frac{a}{2}$, Mn.,$x=a$

344) $n=\frac{x}{x^{2}+1}$

Mn.,$x=0$

345) $n=x+\frac{a^{2}}{x}$

Mx. : $x=1$, Mn.,$x=-1$

346) $\quad n=\frac{a^{3}}{x^{2}}+\frac{1}{(1-x)^{2}}$

Mx.,$x=-a$, Mn.für $x=a$

347) $"=\frac{x}{x^{2}+2 x+4}$

348) $"=\frac{x}{a x^{2}-b x+c}$

Mn.,$x=\frac{a}{a+1} ; a>0$

Mx. , $x=2$

Mn.,$x=-2$

Mx. , $x=\sqrt{\frac{c}{a}}$

Mn. , $x=-\sqrt{\frac{c}{a}}$ 
349) $f(x)=\frac{x^{2}-3 x+2}{x^{2}+3 x+2}$

350) $"= \pm \sqrt{2 a x-x^{2}}$

$351),=\sqrt{2 a x^{2}(2 a-x)}$

352) $"=x+\sqrt{1-x}$

353) $\eta=x \sqrt{2-x^{2}}$

354) $"=x \sqrt{a x-x^{2}}$

355) $"=(a+x) \sqrt{a^{2}-x^{2}}$

356) $"=e^{\frac{x}{a}}+e^{-\frac{x}{a}}$

357) $"=\frac{x^{2}}{e^{x}}$

358) $\quad n=\frac{a^{x}}{x}$

359) $"=x e^{\frac{1}{x}}$

360),$=e^{2 x}-e^{x}$

361) $"=x^{x}$

362) $n=x^{\frac{1}{x}}$

363) $"=\frac{x}{\lg x}$

364) $n=x^{1-\lg x}$

365) $"=2 \sin x+\sin 2 x$
Mx. für $x=-\frac{\sqrt{2}}{2}$
Mn. , $x=\sqrt{2}$

Mx. " $x=a$, Mn. für $x=a$

Mx.,$x=\frac{4 a}{3}$, Mn.,$\quad x=0$

Mx. \# $x=\frac{3}{4}$

Mx. , $x=1$, Mn. für $x=-1$

Mx.,$x=\frac{3 a}{4}$

Mx.,$x=\frac{a}{2}$

Mn.,$x=0$

Mx. , $x=2$, Mn. für $x=0$

Mn.,$x=\frac{1}{\lg a} ; a>1$

Mn.,$x=1$

Mn.,$x=\lg \frac{1}{2}$

Mn.,$x=\frac{1}{e}$

Mx.,$x=e$

Mn.,$x=e$

Mx.,$x=\sqrt{e}$

Mx. , $x=\frac{\pi}{3}$

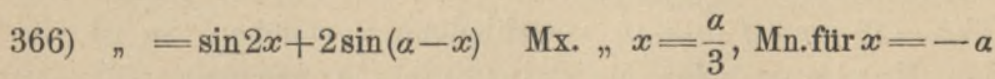
367) $"=\frac{\cos ^{2} x}{a^{2}}+\frac{\sin ^{2} x}{b^{2}}, a>b \quad$ Mx. , $x=\frac{\pi}{2}$, Mn.,$x=0$ 368) $\quad \pi=\cos x+\cos (\alpha-x) ; \quad \alpha<\pi$

Mx.für $x=\frac{\alpha}{2}$ 
369) $f(x)=\sin x \cdot \sin (\alpha+x)$

370) $n=\sin x \cdot \cos (\alpha-x)$

371) $n=e^{x} \cdot \cos x$

372) $n=\frac{e^{x}}{\sin x}$
$\mathrm{Mx}$. für $x=\frac{\pi-a}{2}$

Mn. für $x=\pi-\frac{\alpha}{2}$ Mx.,$x=\frac{\alpha}{2}+\frac{\pi}{4}$,

Mn. für $x=\frac{\alpha}{2}+\frac{3 \pi}{4}$ Mx. , $x=\frac{\pi}{4}$, Mn. für $x=\frac{5 \pi}{4}$ Mn.,$x=\frac{\pi}{4}$, Mx.,$x=\frac{5 \pi}{4}$.

Bisher haben wir nur solche Maxima und Minima behandelt, bei denen der Differentialquotient der Funktion endlich ist und sich stetig ändert. Es kann aber auch der Fall vorkommen, dass die Funktion $y=f(x)$ von $x=a-\delta$ bis $x=a+\delta$ endlich und stetig verläuft, der Differentialquotient $f^{\prime}(x)$ - dagegen an der Stelle $x=a$ eine Unterbrechung der Stetigkeit erleidet. Hier haben wir $\lim f^{\prime}(a-\delta)$ zu unterscheiden von $\lim f^{\prime}(a+\delta)$ bei unendlich kleinem $\delta$. Wir können diese Ausdrücke ohne Missverständnisse zu befürchten ktirzer durch $f^{\prime}(a-0)$ und $f^{\prime}(a+0)$ bezeichnen. Wir verstehen also unter $f^{\prime}(a-0)$ den Grenzwert, welchem sich $f^{\prime}(x)$ unaufhörlich annähert, wenn man $x$ von kleineren Werten aus stetig wachsend an die Stelle $x=a$ heranriteken lässt. Dagegen ist $f^{\prime}(a+0)$ der Grenzwert der Funktion $f^{\prime}(x)$ für den Fall, dass man $x$ von grösseren Werten aus stetig abnehmend an die Stelle $x=a$ bringt. Haben nun $f^{\prime}(a-0)$ und $f^{\prime}(a+0)$ verschiedene Vorzeichen, so ist $f(a)$ ein Maximum oder ein Minimum, je nachdem $f^{\prime}(a-0)$ positiv oder negativ ist.

Beispiel: Es sei $x=a(t-\sin t), y=a(1-\cos t)$, also $\frac{d y}{d x}=\operatorname{cotg} \frac{1}{2} t$ und $\frac{d^{2} y}{d x^{2}}=-\frac{1}{4 a\left(\sin \frac{1}{2} t\right)^{4}}$. Die Funktion $y$ hat 
ein Maximum $y=2 a$ mit stetiger Änderung von $\frac{d y}{d x}$ an der Stelle $t=\pi, x=a \pi$, dagegen ein Minimum $y=0$ mit unstetiger Änderung von $\frac{d y}{d x}$ an der Stelle $t=0, x=0$. Hier ist $\frac{d y}{d x}=-\infty$ für $x=-0$ und $\frac{d y}{d x}=+\infty$ für $x=+0$. Periodische Wiederholung mit der Periode $t=2 \pi$.

Unentwickelte Funktionen. Soll aus einer Gleichung $f(x, y)=0$ ein Maximal- oder ein Minimalwert für $y$ hervorgehen, so muss die unabhängige Variabele $x$ so gewählt werden, dass die Bedingung befriedigt ist $\frac{d y}{d x}=0$. Da nun $\frac{d y}{d x}=-\frac{\partial f}{\partial x}: \frac{\partial f}{\partial y}$, so geht diese in die andere Bedingung über $\frac{\partial f}{\partial x}=0$. Im allgemeinen werden in dieser Gleichung die beiden Variabelen enthalten sein. In Verbindung mit $f(x, y)=0$ giebt sie die gesuchten Werte von $x$ und die zugehörigen Werte von $y$. Wenn für ein solches zusammengehöriges Wertpaar $x$ und $y$ nicht nur $\frac{\partial f}{\partial x}=0$, sondern zugleich auch $\frac{\partial f}{\partial y}=0$ wird, so ist $\frac{d y}{d x}$ nicht mehr gleich Null, sondern unbestimmt, ein Fall, der später eingehend behandelt werden wird. Hier setzen wir voraus, dass $\frac{\partial f}{\partial y}$ nicht verschwinde. Unter den angeführten Umständen reduziert sich der zweite Differentialquotient auf den Ausdruck $\frac{d^{2} y}{d x^{2}}=-\frac{\partial^{2} f}{\partial x^{2}}: \frac{\partial f}{\partial x}$ nach Formel (24), S. 53.

Beispiel: $f=y^{2}+2 x^{2} y+4 x-3=0$;

$$
\frac{\partial f}{\partial x}=4 x y+4=0, \frac{\partial f}{\partial y}=2 y \cdot+2 x^{2} \text {. }
$$

Die beiden ersten Gleichungen ergeben $x=1, y=-1$ und $x=-\frac{1}{2}, y=2$. Da für das erste Wertpaar auch $\frac{\partial f}{\partial y}=0$ 
wird, so ist $y$ weder Maximum noch Minimum. Dagegen wird für das zweite Wertpaar $\frac{d^{2} y}{d x^{2}}=-\frac{16}{9}$, mithin ist $y=2$ ein Maximum.

373) $f=\frac{x^{2}}{a^{2}}+\frac{y^{2}}{b^{2}}-1=0$;

\section{Aufgaben.}

Für $x=0$ wird $y=b$ ein Max., $y=-b$ ein Min.

374) $f=\left(x^{2}+y^{2}\right)^{2}-a^{2}\left(x^{2}-y^{2}\right)=0$.

Für $x=0$ wird $y=0$ weder Max. noch Min., für $x= \pm \frac{a}{2} \sqrt{\frac{3}{2}}$

wird $y=\frac{a}{4} \sqrt{2}$ ein Max. und $y=-\frac{a}{4} \sqrt{2}$ ein Min.

375) $f=x^{3}-3 a^{2} x+y^{3}=0$;

$x=a$ giebt $y=a \sqrt{2}$ als Max. und $x=-a$ gieht

$y=-a \sqrt[3]{2}$ als Min.

376) $f=4 x^{2}-20 x-8 y+41=0$;

$x=\frac{5}{2}$ macht $y=2$ zu einem Min.

377) $f=(y-x)^{3}+x+6=0$;

$x=-6-\frac{1}{3 \sqrt{3}}$ macht $y=-6+\frac{2}{3 \sqrt{3}}$ zu einem Max.,

$x=-6+\frac{1}{3 \sqrt{3}}$ macht $y=-6-\frac{2}{3 \sqrt{3}}$ zu einem Min.

378) $f=y^{2}-a y-\sin x=0$; $a>2$;

$x=\frac{\pi}{2}$ macht $y=\frac{1}{2} a+\frac{1}{2} \sqrt{a^{2}+4}$ zu einem Max. und $y=\frac{1}{2} a-\frac{1}{2} \sqrt{a^{2}+4} \mathrm{zu}$ einem Min.

Was findet sich für $x=-\frac{\pi}{2}$ ?

Relative Maxima und Minima. Gegeben ist die Funktion $z=f(x, y)$ und weiter zwischen $x$ und $y$ die Gleichung $\varphi(x, y)=0$. Es sollen nun $x$ und $y$ so bestimmt werden, 
dass sie der Gleichung $\varphi(x, y)=0$ gentigen und zugleich $z$ $\mathrm{zu}$ einem Maximum oder einem Minimum machen.

Beispiel: In den Kreis $\varphi(x, y)=x^{2}+y^{2}-r^{2}=0$ das an Fläche grösste Rechteck zu zeichnen, dessen Seiten den Koordinatenachsen parallel laufen. Sind $x, y$ die Koordinaten des Eckpunktes im ersten Quadranten und also beide positiv, so ist $z=4 x y$ der Wert, welcher ein Max. werden soll, und zugleich müssen $x$ und $y$ die Bedingung befriedigen: $\varphi(x, y)=x^{2}+y^{2}-r^{2}=0$, weil der Punkt zur Kreislinie gehört. Hier kann leicht $y=\sqrt{r^{2}-x^{2}}$ aus der Gleichung $\varphi=0$ genommen und in $z=4 x y$ substituiert werden; man findet dann, dass $z=4 x \sqrt{r^{2}-x^{2}}$ für $x=r \sqrt{\frac{1}{2}}$ ein Maximum wird.

In vielen Fällen ist jedoch eine solche Substitution äusserst schwierig, wenn nicht ganz unmöglich, so dass die Aufgabe auf andere Weise gelöst werden muss. Da $y$ durch die Gleichung $\varphi=0$ von $x$ abhängt, so muss $x$ als die einzige unabhängige Variabele angesehen werden, und es müssen die Werte von $x$, welche $z$ zu einem Maximum oder einem Minimum machen, der Bedingung $\frac{d z}{d x}=0$ gentigen, d. h. es muss sein

$$
\frac{d z}{d x}=\frac{\partial f}{\partial x}+\frac{\partial f}{\partial y} \cdot \frac{d y}{d x}=0 .
$$

Aus der Gleichung $\varphi=0$ ergiebt sich:

$$
\frac{\partial \varphi}{\partial x}+\frac{\partial \varphi}{\partial y} \cdot \frac{d y}{d x}=0
$$

und durch Elimination von $\frac{d y}{d x}$ aus (A) und (B) entsteht:

$$
\begin{aligned}
& \frac{d z}{d x}=\frac{\frac{\partial f}{\partial x} \cdot \frac{\partial \varphi}{\partial y}-\frac{\partial f}{\partial y} \cdot \frac{\partial \varphi}{\partial x}}{\frac{\partial \varphi}{\partial y}}=0 \\
& \text { oder: } \frac{\partial f}{\partial x} \cdot \frac{\partial \varphi}{\partial y}-\frac{\partial f}{\partial y} \cdot \frac{\partial \varphi}{\partial x}=0
\end{aligned}
$$


Aus (C) und $\varphi=0$ findet man jetzt die Werte für $x$ und $y$, durch welche $z$ ein Maximum oder ein Minimum wird, und die weitere Frage, ob Maximum oder Minimum, wird wieder durch das Vorzeichen von $\frac{d^{2} z}{d x^{2}}$ entschieden, welcher Ausdruck unten noch gebildet wird.

Dieses Verfahren lässt übrigens folgende Abänderung zu. Die Bedingung $(C)$ kann auch durch die folgenden Gleichungen

$$
\frac{\partial f}{\partial x}+\lambda \frac{\partial \varphi}{\partial x}=0, \frac{\partial f}{\partial y}+\lambda \frac{\partial \varphi}{\partial y}=0
$$

ausgedrtickt werden, wenn $\lambda$ eine willkürliche Konstante bedeutet, durch deren Elimination (D) wieder in (C) tibergeht. Tritt aber die weitere Gleichung $\varphi=0$ noch hinzu, so haben wir 3 Gleichungen zwisohen den 3 Unbekannten $x, y$ und $\lambda$. Da nun weiter

$$
\frac{\partial f}{\partial x}+\lambda \frac{\partial \varphi}{\partial x}=\frac{\partial(f+\lambda \varphi)}{\partial x}, \frac{\partial f}{\partial y}+\lambda \frac{\partial \varphi}{\partial y}=\frac{\partial(f+\lambda \varphi)}{\partial y}
$$

ist, so dienen zur Bestimmung von $x, y$ und $\lambda$ die folgenden 3 Gleichungen:

$$
\frac{\partial(f+\lambda \varphi)}{\partial x}=0, \frac{\partial(f+\lambda \varphi)}{\partial y}=0, \varphi(x, y)=0 .
$$

Es bleibt noch tibrig, $\frac{d^{2} z}{d x^{2}}$ abzuleiten. Aus (A) und (B) entsteht:

$$
\begin{aligned}
\frac{d^{2} z}{d x^{2}} & =\frac{d}{d x}\left[\frac{\partial f}{\partial x}+\frac{\partial f}{\partial y} \cdot \frac{d y}{d x}\right] \\
0 & =\frac{d}{d x}\left[\frac{\partial \varphi}{\partial x}+\frac{\partial \varphi}{\partial y} \cdot \frac{d y}{d x}\right] .
\end{aligned}
$$

Wird die letzte Gleichung mit $\lambda$ multipliziert und zur vorhergehenden addiert, so erhält man:

$$
\frac{d^{2} z}{d x^{2}}=\frac{d}{d x}\left[\frac{\partial(f+\lambda \varphi)}{\partial x}+\frac{\partial(f+\lambda \varphi)}{\partial y} \cdot \frac{d y}{d x}\right] .
$$

Führt man rechts die Differentiation durch, so ersoheint $\frac{d^{2} y}{d x^{2}}$ mit dem Faktor $\frac{\partial(f+\lambda \varphi)}{\partial y}$ multipliziert. Bei den hier in 
Betracht kommenden Werten versohwindet dieser nach (27), so dass wir für unsern Fall schreiben können:

$$
\begin{aligned}
\frac{d^{2} z}{d x^{2}}=\frac{\partial^{2}(f+\lambda \varphi)}{\partial x^{2}}+ & \frac{\partial^{2}(f+\lambda \varphi)}{\partial y \partial x} \cdot \frac{d y}{d x} \\
& +\left[\frac{\partial^{2}(f+\lambda \varphi)}{\partial x \partial y}+\frac{\partial^{2}(f+\lambda \varphi)}{\partial y^{2}} \cdot \frac{d y}{d x}\right] \frac{d y}{d x}
\end{aligned}
$$

Wird endlich $\frac{d y}{d x}=-\frac{\partial \varphi}{\partial x}: \frac{\partial \varphi}{\partial y}$ gesetzt, so geht daraus hervor:

$$
\frac{d^{2} z}{d x^{2}}=\frac{\frac{\partial^{2}(f+\lambda \varphi)}{\partial x^{2}}\left[\frac{\partial \varphi}{\partial y}\right]^{2}-2 \frac{\partial^{2}(f+\lambda \varphi)}{\partial x \partial y} \cdot \frac{\partial \varphi}{\partial x} \frac{\partial \varphi}{\partial y}+\frac{\partial^{2}(f+\lambda \varphi)}{\partial y^{2}}\left[\frac{\partial \varphi}{\partial x}\right]^{2}}{\left[\frac{\partial \varphi}{\partial y}\right]^{2}} .
$$

Da der Nenner dieses Wertes ohne Einfluss auf das Vorzeichen ist, so wird $z$ ein Maximum, wenn der Zähler negativ, ein Minimum, wenn der Zähler positiv ist.

Handelt es sich also darum, $z=f(x, y)$ zu einem Max. oder Min. zu machen, während $\varphi(x, y)=0$ ist, dann gilt folgende Regel:

Man bestimme die drei Unbekannten $\lambda, x, y$ so, dass die Gleichungen (27) erfüllt werden. Wenn die erhaltenen Werte den Ausdruck $\frac{d^{2} z}{d x^{2}}$ positiv (negativ) machen, haben wir ein Minimum (Maximum).

1. Beispiel: Für welche Werte von $x$ und $y$ wird $z=x y$ ein Max. oder Min., wenn die Bedingungsgleichung gegeben ist: $\varphi=x^{3}+y^{3}-a x y=0 . \quad f+\lambda \varphi=x y+\lambda\left(x^{3}+y^{3}-a x y\right)$; $\frac{\partial(f+\lambda \varphi)}{\partial x}=y+\lambda\left(3 x^{2}-a y\right)=0 ; \frac{\partial(f+\lambda \varphi)}{\partial y}=x+\lambda\left(3 y^{2}-a x\right)=0$.

Aus den vorstehenden Gleichungen folgt $x=y$, und dann aus $\varphi=0$ weiter:

$$
\begin{gathered}
x=y=\frac{a}{2}, \text { und endlich } \lambda=-\frac{2}{a}, z=\frac{a^{2}}{4} \\
\frac{\partial^{2}(f+\lambda \varphi)}{\partial x^{2}}=6 \lambda x=-6, \frac{\partial^{2}(f+\lambda \varphi)}{\partial y^{2}}=6 \lambda y=-6,
\end{gathered}
$$




$$
\frac{\partial^{2}(f+\lambda \varphi)}{\partial x \partial y}=1-\lambda a=3 ; \frac{\partial \varphi}{\partial x}=3 x^{2}-a y=\frac{a^{2}}{4},
$$

$\frac{\partial \varphi}{\partial y}=3 y^{2}-a x=\frac{a^{2}}{4} ; \frac{d^{2} z}{d x^{2}}=-18$, folglich $z=\frac{a^{2}}{4}$ ein Max.

2. Beispiel: Aus einer Seite $a$ und dem gegenüber liegenden Winkel $\alpha$ soll das an Fläche grösste Dreieck gezeichnet werden. Nennen wir die an der Seite $a$ liegenden Winkel $x$ und $y$, so ist $F=\frac{a^{2} \sin x \sin y}{2 \sin \alpha}$. Da nun ein aus konstanten und variabelen Faktoren zusammengesetztes Produkt seinen grössten oder kleinsten Wert annimmt, wenn das Produkt der variabelen Faktoren am grössten oder kleinsten wird, so dürfen wir $a^{2}$ und $2 \sin \alpha$ unterdrücken und $z=f(x, y)=\sin x \sin y$ als diejenige Funktion ansehen, welche durch passende Wahl von $x$ und $y$ zu einem Max. wird. Zugleich besteht die Relation $\varphi=x+y+\alpha-\pi=0$, so dass $f+\lambda \varphi=\sin x \sin y$ $+\lambda(x+y+\alpha-\pi), \quad \frac{\partial(f+\lambda \varphi)}{\partial x}=\cos x \sin y+\lambda=0$, $\frac{\partial(f+\lambda \varphi)}{\partial y}=\sin x \cos y+\lambda=0$ ist, woraus leicht $\sin (x-y)=0$, oder auch $x-y=0$ erhalten wird, weil die andere Relation $x-y=\pi$ hier nicht zulässig ist. Für $y=x$ wird dann weiter. $\frac{\partial^{2}(f+\lambda \varphi)}{\partial x^{2}}=-\sin ^{2} x, \quad \frac{\partial^{2}(f+\lambda \varphi)}{\partial y^{2}}=-\sin ^{2} x, \quad \frac{\partial^{2}(f+\lambda \varphi)}{\partial x \partial y}$ $=\cos ^{2} x$, und da $\frac{\partial \varphi}{\partial x}=1, \frac{\partial \varphi}{\partial y}=1$ ist, so wird für $y=x$ der Wert $\frac{d^{2} z}{d x^{2}}$ negativ und die Fläche ein Max. Unter allen möglichen Dreiecken mit gegebener Basis und gegebenem Winkel an der Spitze besitzt das gleichschenkelige die grösste Fläche.

Eine andere Aufgabe, namlich die: In einen gegebenen Kreisabschnitt das grösste Dreieck zu zeichnen, fällt mit dieser genau zusammen. 


\section{Aufgaben.}

379). Wie gross muss der Winkel an der Basis eines gleichschenkeligen Dreiecks in gegebenem Kreise gewählt werden, wenn die Fläche des Dreiecks ein Max. werden soll?

Wenn $r$ der Halbmesser des Kreises und $x$ der gesuchte Winkel ist, so ist $F=4 r^{2} \sin ^{3} x \cos x$ und wird Max. für $\cos x=\frac{1}{2}$, oder $x=60^{\circ}$. Im Kreise hat das gleichseitige Dreieck die grösste Fläche.

380) In einem Dreieck ist die Basis $=a$ und der Winkel an der Spitze $=\alpha$ gegeben. Wie ist das Dreieck zu zeichnen, wenn sein Umfang ein Max. werden soll?

Sind $x$ und $y$ die beiden anderen Seiten, so ist:

$$
f=x+y+a, \quad \varphi=x^{2}+y^{2}-2 x y \cos \alpha-a^{2}=0 .
$$

Man findet $f$ als Max. für $x=y$.

381) Eine Strecke $a$ ist so in zwei Teile zu teilen, dass das Produkt aus den Längen der Abschnitte ein Max. wird. $z=x y, \varphi=x+y-a=0 ; z$ wird Max. für $y=x=\frac{a}{2}$.

382) Die Summe der beiden Katheten eines rechtwinkeligen Dreiecks ist konstant, nämlich $x+y=2 s$. Wie sind die Katheten zu wählen, wenn die Hypotenuse $z$ ein Min. werden soll?

$$
z=\text { Min. für } x=y \text {. }
$$

383) Zwischen den Schenkeln eines Winkels $\alpha$, dessen Scheitel $A$ ist, liegt ein Punkt $M$. Man soll dureh $M$ eine Gerade ziehen, welche die Schenkel in $B$ und $C$ sohneidet und zwar so, dass das Dreieck $A B C$ ein Min. wird.

Wir ziehen durch $M$ zu $A C$ eine Parallele, welche $A B$ in $D$ schneidet. Gegeben: $A D=a, M D=b$; gesetzt: $A B=x, \quad A C=y . \quad F=\frac{1}{2} x y \sin \alpha$, oder $z=x y ; \quad \varphi=\frac{a}{x}+\frac{b}{y}-1=0 . \quad F=$ Min. für $x=2 a$, oder $M B=M C$.

384) In der Basis $A B$ eines Dreiecks $A B C$ ist ein Punkt $M$ gegeben; eine Parallele zu $A B$ soll $A C$ in $D$ und $C B$ 
in $E$ schneiden. In welcher Entfernung von der Basis muss $D E$ gezogen werden, wenn das Dreieck $D E M$ ein Max. sein soll?

Wir setzen Basis $A B=c$, Höhe des Dreiecks $A B C=h$, Basis $D E=y$, Höhe des Dreiecks $D M E=x$ und haben: $F=\frac{1}{2} x y, \varphi=c x+h y-c h=0 ; F=$ Max. für $x=\frac{1}{2} h$.

385) Die Grundlinie $b$ und die Höhe $h$ eines Dreiecks sind gegeben. Wie ist das Dreieck zu zeichnen, wenn die Summe der beiden anderen Seiten ein Min. werden soll? Sind $x$ und $y$ die Winkel an der Basis, so ist $z=\frac{h}{\sin x}+\frac{h}{\sin y}$ die Seitensumme, $b h=2 F=\frac{b^{2} \sin x \sin y}{\sin (x+y)}$ oder $\varphi=\operatorname{cotg} x+\operatorname{cotg} y-\frac{b}{h}=0$. Für $x=y$ wird $z$ ein Min.

386) Auf den Seiten $A B$ und $B C$ eines Dreiecks sollen die Punkte $D$ und $E$ so gefunden werden, dass ihre Verbindungslinie $D E$ das Dreieck halbiert und ein Min. wird.

$$
B D=x, B E=y, B A=c, B C=a ;
$$

$D E^{2}=x^{2}+y^{2}-2 x y \cos B, \varphi=x y-\frac{a c}{2}=0$. Man fin$\operatorname{det} z=x^{2}+y^{2}-a c \cos B=$ Min., wenn $x=y=\sqrt{\frac{a b}{2}}$.

Die Aufgabe, "von einem Dreieck $D B E$ ist der Inhalt und der Winkel an der Spitze gegeben, die Grundlinie soll ein Min. werden", fällt mit der vorstehenden zusammen.

387) Eine Gerade $D E$ und 2 auf der nämlichen Seite gelegene Punkte $A$ und $B$ sind gegeben, man soll in $D E$ einen Punkt $C$ so finden, dass $A C+C B$ ein Min. wird.

Man ziehe $A P$ und $B Q$ senkrecht auf $D E$; setze $A P=p, B Q=q, P Q=c ; \Varangle A C P=x, \Varangle B C Q=y$, so ist $z=A C+B C=\frac{p}{\sin x}+\frac{q}{\sin y}, \quad \varphi=p \operatorname{cotg} x+$ $q \operatorname{cotg} y-c=0 ; z$ wird Min. für $x=y$. 
Ist $A C B$ der Weg eines Lichtstrahls, so liefert die Lösung dieser Aufgabe das Gesetz der Reflexion durch die Forderung, dass der Weg ein Min. sein solle.

388) Ein Winkel $B$ und eine Strecke $D E=b$ sind gegeben. Es soll $b$ so in den Winkel eingetragen werden, dass die Endpunkte $D$ und $E$ auf die Schenkel des Winkels fallen und das Dreieck $D B E$ ein Max. wird.

Es sei $B D=x, B E=y ; \quad F=\frac{1}{2} x y \sin B, \varphi=x^{2}$ $+y^{2}-2 x y \cos B-b^{2}=0 . \quad F=$ Max. für $x=y$.

Man vergleiche den Zusatz zu 386). - Die Aufgabe bietet eine andere Fassung und andere Lösung von Beispiel 2.

389) Gegeben ist ein rechter Winkel mit dem Scheitel $A$ und auf dem einen Schenkel die Punkte $B$ und $C$. Man soll auf dem andern Schenkel einen Punkt $D$ so finden, dass Winkel $B D C=v$ möglichst gross wird.

$$
A B=b, \quad A C=c, A D=x, \Varangle A B D=\beta,
$$

$\gamma A C D=\gamma ; \quad \operatorname{tg} \beta=\frac{x}{b}, \quad \operatorname{tg} \gamma=\frac{x}{c} ; \quad \operatorname{tg} v=\frac{(c-b) x}{b c+x^{2}}$ wird mit $v$ selbst Max. für $x= \pm \sqrt{b c}$.

390) Welches von allen Vierecken, die aus den 4 gegebenen Seiten $a, b, c, d$ konstruiert werden können, hat die grösste Fläche?

Ist $x$ der von $a$ und $b, y$ der von $c$ und $d$ eingeschlossene Winkel, so ist $2 F=a b \sin x+c d \sin y$.

Durch die Diagonale wird die Relation vermittelt:

$\varphi=2 a b \cos x-2 c d \cos y-a^{2}-b^{2}+c^{2}+d^{2}=0$.

Man findet, dass für $\sin (x+y)=0$, d. h. $x+y=\pi$ $F$ ein Max. wird. Das Viereck ist also ein Kreisviereck.

391) In eine Ellipse soll das grösste Rechteck gezeichnet werden, dessen Seiten den Achsen der Ellipse parallel laufen.

Gl. der El.: $x=a \cos t, y=b \sin t$; $F=4 x y=2 a b \sin 2 t=$ Max. für $t=45^{\circ}$, oder: $x=a \sqrt{\frac{1}{2}}, y=b \sqrt{\frac{1}{2}}$. 
392) Wie ist der Halbmesser eines Kreises zu wählen, wenn ein Sektor von gegebenem Umfang $=2 a$ die grösste Fläche besitzen soll?

Ist der Halbmesser $=x$ und der Bogen $=2 y$, so ist $x+y=a$ und $F=x y$ ein Max. für $x=\frac{a}{2}$.

393) Ein Parabelsegment wird durch eine zur Achse senkrechte Sehne $B C$ begrenzt, deren Abstand vom Scheitel $A$ gleich $a$ ist. Eine zweite Sehne $M N$ soll parallel der ersten innerhalb des Segmentes so gezogen werden, dass das Rechteck, dessen eine Seite $M N$ ist, und dessen Gegenseite in $B C$ liegt, die grösste Fläche besitzt.

Sind $x, y$ die Koordinaten des gesuchten Punktes $M$, so ist $F=y(a-x)$ und $y^{2}=2 p x ; F=\operatorname{Max}$. für $x=\frac{a}{3}$.

394) Die vorhergehenden Angaben; jetzt soll der Umfang des Rechtecks ein Min. werden.

$\frac{1}{2} U=y+a-x ; y^{2}=2 p x$. Man findet $U=$ Min. für $y=p, x=\frac{1}{2} p$.

395) Zwei Tangenten eines Kreises bilden einen Winkel $=2 \alpha$. Wie ist eine dritte Tangente zu legen, wenn das dem Kreise umschriebene Tangentendreieck ein Min. werden soll?

Die Winkel, welche die dritte Tangente mit den gegebenen bildet, mögen $2 x$ und $2 y$ sein; dann ist:

$$
F=r^{2}(\operatorname{cotg} x+\operatorname{cotg} y+\operatorname{cotg} \alpha) \text { und }
$$

$\varphi=x+y+\alpha-90^{\circ}=0$. F wird Min. für $x=y$.

396) In einem Kreise ist eine Sehne $A B$ gegeben, und es soll im kleineren Abschnitt zu ihr parallel eine zweite Sehne $C D$ so gezogen werden, dass das Paralleltrapez $A B C D$ ein Max. wird.

Es sei $M$ der Mittelpunkt, $M H$ der zu $A B$ senkrechte Halbmesser, $\Varangle A M H=\alpha, \Varangle C M H=x$, so ist $2 F=r^{2}(\sin x+\sin \alpha)(\cos x-\cos \alpha)$ und wird Max. für 
$x=\frac{\alpha}{3}$, wie aus der Bestimmungsgleichung $\cos 2 x$ $=\cos (x-\alpha)$ folgt.

397) Es soll bewiesen werden, dass der Kreis als das reguläre Vieleck angesehen werden kann, welches bei konstantem Umfange die grösste Fläche besitzt.

Ist $x$ die Seitenzahl und $P$ der Umfang des Vielecks, dann wird seine Fläche $F=\frac{P^{2}}{4 x} \operatorname{cotg} \frac{\pi}{x}$ ein Max., wenn $\sin \frac{2 \pi}{x}=\frac{2 \pi}{x}$, d. h. $x=\infty$ ist.

398) Ein gerader Kreiskegel, dessen Spitze $A$ heisst und dessen Basisdurchmesser $B C=2 r$ und dessen Seitenkante $A B=s$ ist, soll durch eine zu $A C$ parallele Ebene so geschnitten werden, dass der Inhalt der entstehenden Parabelfläche ein Max. ist.

Es sei $S$ der auf $A B$ gelegene Scheitel, $S D$ die zu $A C$ parallele Achse der Parabel, mithin $D$ ein Punkt von $B C$. Es sei ferner $S D=x$ und die zu $x$ gehörige Ordinate $=y$, so ist $F=\frac{2}{3} x y$ und $y^{2}=B D . D C$ $=D C(2 r-D C)$. Daraus ergiebt sich $y=\frac{2 r}{s} \sqrt{s x-x^{2}}$ und $F$ wird Max. für $x=\frac{3}{4} s$ oder $A S=\frac{1}{4} A B$.

399) Um ein Rechteck soll die an Fläche kleinste Ellipse beschrieben werden.

$a$ und $b$ die halben Reohteckseiten, $x$ und $y$ die Halbachsen. $F=x y \pi$ und $\varphi=\frac{a^{2}}{x^{2}}+\frac{b^{2}}{y^{2}}-1=0$. Es wird $F=$ Min. für $x=a \sqrt{2}, y=b \sqrt{2}$. Vgl. 391).

400) Um ein gleichschenkeliges Dreieck soll die an Fläche kleinste Ellipse gezeichnet werden, deren kleine Achse parallel zur Grundlinie des Dreiecks läuft.

Spitze des Dreiecks $A$, Basis $B C=2 n$, Höhe $=h$. Sind $u$ und $v$ die Halbachsen, so heisst die Scheitelgleichung der Fllipse: $u^{2} y^{2}=v^{2}\left(2 u x-x^{2}\right)$ und so be- 
steht die Relation: $u^{2} n^{2}=v^{2}\left(2 u h-h^{2}\right)$. Die Fläche $F=u v \pi$ wird ein Min. für $u=\frac{2}{3} h$.

401) In ein gleichschenkeliges Dreieck soll eine Ellipse so gezeichnet werden, dass sie die Seiten des Dreiecks berühre, dass ihre kleine Achse parallel zur Grundlinie des Dreiecks laufe und dass ihre Fläche ein Max. sei.

$A B=2 n$ die Basis, $C$ Spitze, $D C=h$ Höhe und zugleich $X$-Achse, $A B Y$-Achse, $u$ und $v$ Halbachsen. Nun ist $u^{2} y^{2}=v^{2}\left(2 u x-x^{2}\right)$ die Scheitelgleichung der Ellipse und $y=-\frac{n}{h} x+n$ die Gleichung von $A C$. Suchen wir die Abscissen der Schnittpunkte zwischen der Ellipse und $A C$ und setzen den Wurzelteil gleich Null, so ist dies die Bedingung dafür, dass die Gerade die Kurve berührt. Sie heisst: $v^{2}+\frac{2 n^{2} u}{h}-n^{2}=0 ; \quad F=u v \pi$ wird Max. für $u=\frac{h}{3}$.

402) In ein gleichschenkeliges Dreieck soll eine Parabel so gezeichnet werden, dass ihre Achse auf der Mitte der Grundlinie rechtwinkelig stehe, dass sie die beiden gleichen Seiten berühre und das von der Basis begrenzte Parabelsegment ein Max. sei.

Es sei $A$ die Spitze, $h$ die Höhe, $B C=2 n$ die Basis des Dreiecks, $A$ der Anfangspunkt der Koordinaten, $S$ der Parabelscheitel, $A S=u$. Die Achse der $x$ geht dureh die Mitte der Grundlinie des Dreiecks. Die Parabelgleichung heisst: $y^{2}=2 p(x-u)$, die Gleichung von $A B: y=\frac{n}{h} x=k x$. Als Bedingung dafür, dass $A B$ die Kurve berihre, wird gefunden: $p-2 k^{2} u=0$, worin auch $p$ noch unbekannt ist. Die Fläche $F=\frac{2}{3} \sqrt{2 p(h-u)^{3}}$ wird ein Max. für $u=\frac{h}{4}$. 
403) Ein Kreis ist dureh eine Gleichung $y^{2}=2 r x-x^{2}$ bestimmt. Anfangspunkt und X-Achse des Systems sind zugleich Scheitel und Achse einer Parabel, welche den Kreis in den Punkten $B$ und $C$ schneidet. Wie muss der Parameter $p$ der Parabel gewählt werden, wenn das von $B C$ begrenzte Segment derselben ein Max. werden soll?

Die Koordinaten von $B$ mögen $x$ und $y$ sein, dann ist $F=\frac{2}{3} x y, y^{2}=2 p x$ und $y^{2}=2 r x-x^{2}$, woraus folgt, dass $F=\frac{8}{3} \sqrt{p(r-p)^{3}}$ ein Max. wird für $p=\frac{r}{4}$.

404) Welcher von allen geraden Kreiscylindern, deren Oberfläche $=a^{2}$ ist, besitzt das grösste Volumen?

$x=$ Basishalbmesser, $y=$ Höhe, $V=x^{2} y \pi$, $\varphi=2 \pi x(x+y)-a^{2} ; V$ wird ein Max. für $y=2 x$.

405) Ein gerader Kreiskegel soll parallel zur Basis so geschnitten werden, dass der zwischen der Schnittfläche und der Kegelbasis gelegene Kreiscylinder an Volumen ein Max. ist.

$r=$ Basishalbmesser, $h=$ Höhe des Kegels; $x=$ Basishalbmesser, $y=$ Höhe des Cylinders. $V=x^{2} y \pi$; $\varphi=h x+r y-h r=0 . \quad V$ wird Max. für $x=\frac{2 r}{3}$.

406) Welcher von allen geraden Kreiskegeln von gleicher Seitenlänge $=s$ hat den grössten Kubikinhalt?

Das Volumen wird ein Max., wenn der Basishalbmesser $=s \sqrt{\frac{2}{3}}$ ist.

407) Aus einem Kreise, dessen Halbmesser $r$ ist, soll ein Sektor so geschnitten werden, dass der übrig bleibende Teil der Kreisfläche, als Kegelmantel verbraucht, einen Kegel bestimmt, dessen Volumen ein Max. ist.

Ist $x$ der Halbmesser der Kegelbasis, so wird $V$ ein Max. für $x=r \sqrt{\frac{2}{3}}$.

408) Eine Pyramide soll parallel zur Basis durchschnitten und die Schnittfäche als Endfläche eines Prismas benutzt werden, dessen kongruente Grundfläche in der Basis der 
Pyramide liegt. Wo ist der Schnitt zu fuhren, wenn das Volumen des Prismas ein Max. werden soll?

$B$ Grundfläche, $h$ Höhe der Pyramide; $y$ Grundfläohe, $x$ Höhe des Prismas. Es ist $y: B=(h-x)^{2}: h^{2}$ und $V=x y$ Max. für $x=\frac{h}{3}$. 一(Vgl. die Aufgabe mit 405).

409) Dieselben Angaben, nur soll statt des Prismas eine zweite Pyramide konstruiert werden, deren Grundfläche die Schnittfläche ist und deren Spitze in der Basis der gegebenen Pyramide liegt.

410) Dieselben Angaben, nur sollen statt der beiden Pyramiden 2 Kegel gesetzt werden.

411) In der Achse einer Parabel ist ein Punkt $B$ gegeben und man soll senkrecht zur Achse zwischen $B$ und dem Scheitel $A$ eine Sehne $C D$ so ziehen, dass der Kegel, welchen das Dreieck $B C D$ bei seiner Drehung um die Parabelachse erzeugt, möglichst grosses Volumen habe. Schneidet $C D$ die Achse in $P$ und ist $A B=a, A P=x$, $C P=y$, so ist $y^{2}-2 p x=0$ und $V=\frac{y^{2} \pi(a-x)}{3}$ ein Max. für $x=\frac{a}{2}$.

412) Eine regelmässige Pyramide mit quadratischer Basis soll so in eine Kugel gezeichnet werden, dass ihre Ecken in der Kugeloberfläche liegen und ihr Volumen ein Max. sei.

Ist $x$ der Abstand der Grundfläche von dem Kugelmittelpunkt und $y$ die halbe Seite dieser quadratisohen Grundfläche, so ist $V=\frac{4}{3} y^{2}(r+x)$ und $x^{2}+2 y^{2}-r^{2}=0$. Für $x=\frac{r}{3}$ wird $V$ ein Max.

413) Für welchen Punkt eines Ellipsenquadranten schliessen Durchmesser und Normale den grössten Winkel ein?

Ellipsengleichung $b^{2} x^{2}+a^{2} y^{2}=a^{2} b^{2}$; gesuchter Punkt $x, y ;$ gesuchter Winkel $=\psi ; \operatorname{tg} \psi=\frac{a^{2}-b^{2}}{a^{2} b^{2}} x y$ wird mit $x y$ ein Max. für $x=a \sqrt{\frac{1}{2}}, y=b \sqrt{\frac{1}{2}}$. 
414) Nachzuweisen, dass die Tangente durch den in der vorhergehenden Aufgabe gefundenen Punkt mit den Achsen ein Minimaldreieck einschliesst.

415) Zwei Kugeln liegen ganz auseinander. In welchem Punkte ihrer Centrallinie kann das Max. der Summe der beiden Kugelhauben übersehen werden?

416) Über der Centrallinie zweier Kugeln als Durchmesser ist in irgend einer der hindurohgehenden Ebenen ein Kreis beschrieben. Von welchem Punkte dieses Kreises aus kann die grösste Summe der beiden Kugelhauben ubersehen werden?

\section{§ 11. Die Reihen von Taylor und Maclaurin.}

Mittelwertsatz. Wenn die Funktion $f(x)$ und ihr Differentialquotient $f^{\prime}(x)$ zwischen $x=a$ und $x=b$ uberall stetig ist, und $M$ das Max., $m$ das Min. von $f^{\prime}(x)$ in diesem Intervalle bedeutet, so setzen wir

$$
\begin{aligned}
& \Phi(x)=f(x)-f(a)-M(x-a) \\
& \varphi(x)=f(x)-f(a)-m(x-a) .
\end{aligned}
$$

Dann ist in diesem Intervall

$$
\Phi^{\prime}(x)=f^{\prime}(x)-M<0, \quad \varphi^{\prime}(x)=f^{\prime}(x)-m>0,
$$

also wächst in ihm $\varphi(x)$ ununterbrochen, während $\Phi(x)$ abnimmt (vgl. S. 64). Da nun für $x=a$ die Funktionen $\Phi$ und $\varphi$ verschwinden, so ist $\Phi(b)$ negativ und $\varphi(b)$ positiv, d. h.

$$
f(b)-f(a)-M(b-a)<0, \quad f(b)-f(a)-m(b-a)>0 .
$$

Lässt man also die Variabele $t$ in

$$
f(b)-f(a)-(b-a) f^{\prime}(t)=\psi(t)
$$

das Intervall von $a$ bis $b$ durehlaufen, so wird $\psi$ für $f^{\prime}=M$ negativ und für $f^{\prime}=m$ positiv werden. Folglich giebt es ein $t$ zwischen $a$ und $b$, für welohes $\psi(t)=0$ wird. Bezeichnen wir dies $t$ mit $a+\theta(b-a)$, wobei $\theta$ einen positiven echten Bruch bedeutet, so folgt

$$
f(b)-f(a)=(b-a) f^{\prime}(a+\theta(b-a))(0 \leqq \Theta \leqq 1) .
$$


Dieser Satz heisst der Mittelwertsatz. Wir können ihn geometrisch leicht repräsentieren: Wenn eine Kurve zwischen $A$ und $B$ stetig verläuft

Fig. 10 .

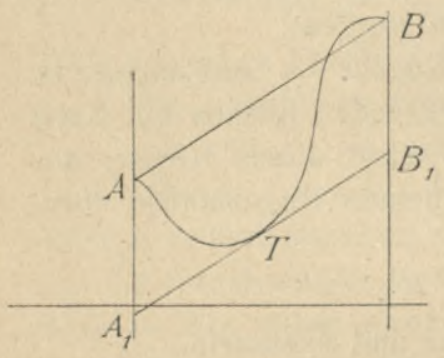
und das Steigungsmass ihrer Tangenten für die gleiche Strecke stetig sich ändert, dann giebt es zwischen $A$ und $B$ mindestens eine Tangente $A_{1} B_{1}$, welche der Sehne $A B$ parallel läuft.

Aus dem Satze folgt: Wenn $f(a)=0$ und $f(b)=0$ ist, dann giebt es für $f^{\prime}(x)=0$ eine Lösung, die zwischen $x=a$ und $x=b$ liegt.

Taylorsche Reihe. Es sei $f(x)$ eine Funktion, die nebst ihren ersten $n$ Ableitungen auf der Strecke von $a$ bis $b$ einwertig, endlich und stetig sein soll. Wir betrachten die Funktion

$$
\begin{aligned}
\phi(x)=f(b)-f(x)- & \frac{b-x}{1} f^{\prime}(x)-\frac{(b-x)^{2}}{1.2} f^{\prime \prime}(x)-\ldots \\
& -\frac{(b-x)^{n-1}}{1.2 \ldots(n-1)} f^{(n-1)}(x)-\frac{(b-x)^{p}}{p} K,
\end{aligned}
$$

in weleher $p$ und $K$ von $x$ unabhängige Grössen sein sollen. Wir wollen $K$ durch eine lineare Gleichung so bestimmt denken, dass $\psi(a)=0$ wird. Die Form von $\psi$ zeigt, dass auch $\psi(b)=0$ wird, wenn $p$ positiv ist. Nach dem Zusatze zum Mittelwertsatze giebt es daher einen Wert $x=\xi$ zwischen $a$ und $b$ für den

$$
\psi^{\prime}(x)=-\frac{(b-x)^{n-1}}{1.2 \ldots(n-1)} f^{(n)}(x)+(b-x)^{p-1} K
$$

versehwindet; d. h. es giebt einen Mittelwert $\xi$, für den

$$
K=\frac{(b-\xi)^{n-p}}{1.2 \ldots(n-1)} f^{(n)}(\xi)
$$


wird. Setzt man dies in $\psi(a)$ ein, so wird, da $\psi(a)=0$,

$$
\begin{aligned}
f(b)= & f(a)+\frac{b-a}{1} f^{\prime}(a)+\frac{(b-a)^{2}}{1.2} f^{\prime \prime}(a)+\ldots \\
& +\frac{(b-a)^{n-1}}{1.2 \ldots(n-1)} f^{(n-1)}(a)+\frac{(b-a)^{p}(b-\xi)^{n-p}}{p .1 .2 \ldots(n-1)} f^{(n)}(\xi) .
\end{aligned}
$$

Statt $a$ und $b$ schreiben wir $x$ und $x+h$, statt $\xi$ wieder $a+(b-a) \Theta=x+h \Theta ;$ dann entsteht

$$
\begin{aligned}
& f(x+h)=f(x)+\frac{h}{1} f^{\prime}(x)+\frac{h^{2}}{1.2} f^{\prime \prime}(x)+\ldots \\
+ & \frac{h^{n-1}}{1.2 \ldots(n-1)} f^{(n-1)}(x)+\frac{h^{n}(1-\theta)^{n-p}}{p \cdot 1.2 \ldots(n-1)} f^{(n)}(x+\theta h) .
\end{aligned}
$$

Das letzte Glied nennt man den Rest der Reihe und schreibt dafür $R$. Setzt man in ihm $p=1$, dann hat man die Lagrangesche Restform

$$
R_{\mathrm{I}}=\frac{h^{n}(1-\theta)^{n-1}}{1.2 \ldots(n-1)} f^{(n)}(x+\theta h),
$$

und für $p=n$ entsteht die Cauchysche Rcstform

$$
R_{\mathrm{II}}=\frac{h^{n}}{1.2 \ldots n} f^{(n)}(x+\theta h) .
$$

(29) ist die Taylorsche Reihe. Sie ist abgeleitet unter der Voraussetzung, dass die Funktion $f(x+h)$ und ihre Differentialquotienten von der ersten bis zur $n^{\text {ten }}$ Ordnung einwertig, endlich und stetig variabel verlaufen auf einem Gebiete, welches die Stelle $h=0$ in sich enthält.

Maclaurinsche Reihe. Wird in (29) statt $x$ geschrieben 0 und $x$ statt $h$, so folgt die Reihe

$$
\begin{gathered}
f(x)=f(0)+\frac{x}{1} f^{\prime}(0)+\frac{x^{2}}{1.2} f^{\prime \prime}(0)+\ldots \\
\quad+\frac{x^{n-1}}{1.2 \ldots(n-1)} f^{(n-1)}(0)+R^{(0)} \\
R^{(0)}=\frac{(1-\theta)^{n-p} x^{n}}{p .1 .2 \ldots(n-1)} f^{(n)}(\theta x)
\end{gathered}
$$


und insbesondere

$$
R_{\mathrm{I}}^{(0)}=\frac{(1-\Theta)^{n-1} x^{n}}{1.2 \ldots(n-1)} f^{(n)}(\Theta x), \quad R_{\mathrm{II}}^{(0)}=\frac{x^{n}}{1.2 \ldots n} f^{(n)}(\Theta x) .
$$

Sie ist gültig, wenn die Funktion $f(x)$ und ihre Differentialquotienten von der ersten bis zur $n^{\text {ten }}$ Ordnung einwertig, endlich und stetig variabel verlaufen auf einem Gebiete, welches die Stelle $x=0$ in sich enthält.

Natürlich fordert die Berechnung von $f^{\prime}(0), f^{\prime \prime}(0) \ldots$, dass zuerst $f^{\prime}(x), f^{\prime \prime}(x) \ldots$ gebildet und darin $x=0$ gesetzt wird.

Ist in (29) $\lim R=0$ für $n=\infty$ und die oben ausgesprochene Bedingung erfüllt für die Funktion und ihre sämmtlichen Differentialquotienten, so darf man die unendliche Reihe ansetzen:

$$
f(a+x)=f(a)+\frac{x}{1} f^{\prime}(a)+\frac{x^{2}}{1.2} f^{\prime \prime}(a)+\frac{x^{3}}{1.2 .3} f^{\prime \prime \prime}(a)+\ldots
$$

Ebenso darf man in (31) die unendliche Reihe nehmen, wenn $\lim R_{\mathrm{I}}=0$ für $n=\infty$ und wenn die oben ausgesprochene Bedingung für die Funktion und ihre sämmtlichen Differentialquotienten erfullt ist. Dann hat man:

$$
f(x)=r^{\prime}(0)+\frac{x}{1} f^{\prime}(0)+\frac{x^{2}}{1.2} f^{\prime \prime}(0)+\frac{x^{3}}{1.2 .3} f^{\prime \prime \prime}(0)+\ldots
$$

Die Bedingung: $\lim R=0$, resp. $\lim R^{(0)}=0$ für $n=\infty$ fordert mehr als die blosse Konvergenz der entstehenden unendlichen Reihen; denn bei blosser Konvergenz kann $R$ sich auch einer von 0 versehiedenen Grenze nähern.

\section{Aufgaben.}

417) Das Binomium. Zu $f(x)=x^{n}$ gehören die Werte $f(a)=a^{n}, \quad f^{\prime}(a)=n a^{n-1}, \quad f^{\prime \prime}(a)=n(n-1) a^{n-2}$, $f^{\prime \prime \prime}(a)=n(n-1)(n-2) a^{n-3}$, usw. Nach der Reihe von Taylor erhält man $f(x+a)$ oder:

$$
\begin{aligned}
(a+x)^{n}=a^{n}+n & a^{n-1} \frac{x}{1}+n(n-1) a^{n-2} \frac{x^{2}}{1.2} \\
& +n(n-1)(n-2) a^{n-3} \frac{x^{3}}{1.2 .3}+\cdots
\end{aligned}
$$


418) Die Reihe für $(1+x)^{-n}$ zu finden. $f(x)=x^{-n}, f(1)=1$, $f^{\prime}(1)=-n, f^{\prime \prime}(1)=n(n+1), f^{\prime \prime \prime}(1)=-n(n+1)(n+2)$ usw.

$(1+x)^{-n}=1-n \frac{x}{1}+n(n+1) \frac{x^{2}}{1.2}-n(n+1)(n+2) \frac{x^{3}}{1.2 .3}+\ldots$

419) Man entwickele den Ausdruck $\sqrt{1+x}$ in eine Reihe.

$f(x)=x^{\frac{1}{2}}, f^{\prime}(x)=\frac{1}{2} x^{-\frac{1}{2}}, f^{\prime \prime}(x)=-\frac{1}{4} x^{-\frac{3}{2}}$, usw.;

daher $f(1)=1, f^{\prime}(1)=\frac{1}{2}, f^{\prime \prime}(1)=-\frac{1}{4}$, usw.

$$
\sqrt{1+x}=1+\frac{x}{2}-\frac{1}{2} \cdot \frac{x^{2}}{4}+\frac{1.3}{2.4} \cdot \frac{x^{3}}{6}-\ldots
$$

420) Man leite die Reihe her:

$$
\frac{1}{\sqrt{1+x}}=1-\frac{1}{2} x+\frac{1.3}{2.4} x^{2}-\frac{1.3 .5}{2.4 .6} x^{3}+\ldots
$$

421) Wenn $f(x)=x^{3}-3 x^{2}+4 x-5$ ist, soll $f(x+2)$ entwickelt werden. Es ist $f(2)=-1, f^{\prime}(2)=4, f^{\prime \prime}(2)=6$, $f^{\prime \prime \prime}(2)=6 ; f(x+2)=-1+4 x+3 x^{2}+x^{3}$.

422) Nach der Formel von Maclaurin soll abgeleitet werden:

$$
\frac{a+x}{b+x}=\frac{a}{b}+\frac{b-a}{b^{2}} x+\frac{a-b}{b^{3}} x^{2}+\frac{b-a}{b^{4}} x^{8}+\ldots
$$

423) Die Reihe für $a^{x}$. $f(x)=a^{x}, f(0)=1, f^{\prime}(0)=\lg \alpha$, $f^{\prime \prime}(0)=(\lg a)^{2}, f^{\prime \prime \prime}(0)=(\lg a)^{3}$, usw.

$$
a^{x}=1+\frac{x}{1} \lg a+\frac{x^{2}}{1.2}(\lg a)^{2}+\frac{x^{3}}{1.2 .3}(\lg a)^{3}+\ldots
$$

Daraus geht die Reihe für $e^{x}$ hervor, wenn man $e$ statt $a$ setzt, wodurch $\lg a$ zu $\lg e=1$ wird.

424) Die Reihe für $\lg (1+x) . \quad f(x)=\lg x, \quad f(1)=0$, $f^{\prime}(1)=1, f^{\prime \prime}(1)=-1, f^{\prime \prime \prime}(1)=2, f^{\prime \prime \prime \prime}(1)=-1.2 .3$, $f^{\text {(V) }}(1)=1 \cdot 2 \cdot 3 \cdot 4$, usw.

$$
\lg (1+x)=x-\frac{x^{2}}{2}+\frac{x^{3}}{3}-\frac{x^{4}}{4}+\frac{x^{5}}{5}-\ldots
$$

Wird darin $-x$ statt $x$ gesetzt, so entsteht:

$$
\begin{aligned}
& \lg (1-x)=-x-\frac{x^{2}}{2}-\frac{x^{3}}{3}-\frac{x^{4}}{4}-\frac{x^{5}}{5} \cdots \\
& \lg \frac{1+x}{1-x}=2\left(\frac{x}{1}+\frac{x^{3}}{3}+\frac{x^{5}}{5}+\cdots\right)
\end{aligned}
$$


Durch Substitution von $x=\frac{1}{2 z+1}$ geht die vorstehende in folgende für die Berechnung bequeme Formel über:

$\lg (z+1)=\lg z+2\left[\frac{1}{2 z+1}+\frac{1}{3} \frac{1}{(2 z+1)^{3}}+\frac{1}{5} \frac{1}{(2 z+1)^{5}}+\ldots\right]$. Aus $a^{x}=y$ folgt $\operatorname{Lg} y=x$ (für die Basis $a$ ); andrerseits ergiebt $e^{x \lg a}=y$ die Beziehung $\lg y=x \lg a$, und daraus

$$
\operatorname{Lg} y=\frac{1}{\lg a} \lg y, \quad \operatorname{Lg}(1+x)=\frac{1}{\lg a} \lg (1+x) .
$$

Will man also $\operatorname{Lg}(1+x)$ in eine Reihe entwiokeln, so kommt man auf die für $\lg (1+x)$ zurück, nur dividiert durch $\lg a$. Die einfachste und natürlichste Entwicklung wird also für $a=e$ stattfinden. Deshalb heissen die zur Basis $e$ gehörigen Logarithmen die natürlichen.

425) Nachzuweisen, dass:

$$
\left(1+e^{x}\right)^{3}=8+12 \frac{x}{1}+24 \frac{x^{2}}{1.2}+54 \frac{x^{3}}{1.2 .3}+\ldots
$$

426) Die Reihe für $\sin (x+a) . \quad f(x)=\sin x, f(a)=\sin a$, $f^{\prime}(a)=\cos a, f^{\prime \prime}(a)=-\sin \alpha$, usw.

$\sin (x+a)=\sin a+\cos a \frac{x}{1}-\sin a \frac{x^{2}}{1.2}-\cos a \frac{x^{3}}{1.2 .3}+\cdots$

Für $a=0$ wird:

$$
\sin x=x-\frac{x^{3}}{1.2 .3}+\frac{x^{5}}{1 \cdot 2 \cdot 3 \cdot 4 \cdot 5}-\ldots
$$

427) Die Reihe für $\cos (x+a) . \quad f(x)=\cos x, f(a)=\cos a$, $f^{\prime}(a)=-\sin a, f^{\prime \prime}(a)=-\cos a, f^{\prime \prime \prime}(a)=\sin a$, usw. $\cos (a+x)=\cos a-\sin a \frac{x}{1}-\cos a \frac{x^{2}}{1.2}+\sin a \frac{x^{3}}{1.2 \cdot 3}+\ldots$ Für $a=0$ erhält man:

$$
\cos x=1-\frac{x^{2}}{1 \cdot 2}+\frac{x^{4}}{1 \cdot 2 \cdot 3 \cdot 4}-\ldots
$$

428) Auf ähnliche Weise entwickelt man:

$\operatorname{tg}(a+x)=\operatorname{tg} a+\frac{1}{\cos ^{2} a} \frac{x}{1}+\frac{2 \operatorname{tg} a}{\cos ^{2} a} \frac{x^{2}}{1.2}+\frac{2\left(1+3 \operatorname{tg}^{2} a\right)}{\cos ^{2} a} \frac{x^{3}}{1.2 .3}+\ldots$

Das Gesetz der Koefficienten ist hier nicht ubersichtlich. 
429) Nach der Formel von Maclaurin soll $f(x)=\cos ^{2} x$ entwickelt werden.

$$
\cos ^{2} x=1-2 \frac{x^{2}}{1.2}+8 \frac{x^{4}}{1.2 \cdot 3 \cdot 4}-32 \frac{x^{6}}{1.2 \ldots .6}+\ldots
$$

430) Mittelst der Reihe von Maclaurin ist die Formel von Moivre abzuleiten. Gegeben ist $f(x)=(\cos x+i \sin x)^{n}$. Man findet: $f^{\prime}(x)=n(\cos x+i \sin x)^{n-1}(-\sin x+i \cos x)=$ $=n i(\cos x+i \sin x)^{n}=n i \cdot f(x)$, und daraus folgt, dass $f^{\prime \prime}(x)=n i \cdot f^{\prime}(x)=(n i)^{2} f(x)$ ist usw. Nun ist $f(0)=1_{4}$ $f^{\prime}(0)=n i, f^{\prime \prime}(0)=-n^{2}, f^{\prime \prime \prime}(0)=-n^{3} i$, usw., und

$$
\begin{aligned}
(\cos x+i \sin x)^{n}=1- & \frac{n^{2} x^{2}}{1.2}+\frac{n^{4} x^{4}}{1.2 .3 .4}-\ldots \\
& \left.+i\left(\frac{n x}{1}-\frac{n^{3} x^{3}}{1.2 .3}+\cdots\right)\right),
\end{aligned}
$$

oder nach den für $\sin x$ und $\cos x$ entwickelten Reihen:

$$
(\cos x+i \sin x)^{n}=\cos (n x)+i \sin (n x) .
$$

431) Die Funktion $f(x)=\operatorname{arc} \sin x$ soll in eine Reihe entwickeit werden. Weil die höheren Differentialquotienten wenig einfache Formen annehmen, benutzen wir die sogenannte Methode der unbestimmten Koefficienten, die stets mit Sicherheit angewendet werden darf, sobald die Möglichkeit der Entwickelung fest steht. Wir setzen:

$$
\arcsin x=A+B x+C x^{2}+D x^{3}+E x^{4}+\ldots
$$

und finden sofort $A=0$ für $x=0$. Wird auf beiden Seiten differentiiert, so entsteht:

$$
\frac{1}{\sqrt{1-x^{2}}}=B+2 C x+3 D x^{2}+4 E x^{3}+\cdots
$$

Für die linke Seite liefert Aufgabe 420) die Reihe:

$$
\left(1-x^{2}\right)^{-\frac{1}{2}}=1+\frac{1}{2} x^{2}+\frac{1.3}{2.4} x^{4}+\frac{1.3 .5}{2.4 .6} x^{6}+\cdots
$$


und so erhält man durch Vergleichung:

$$
B=1, \quad C=0, \quad D=\frac{1}{2.3}, \quad E=0 \text {, usw., }
$$

$\arcsin x=x+\frac{1}{2} \frac{x^{3}}{3}+\frac{1.3}{2.4} \frac{x^{5}}{5}+\frac{1.3 .5}{2.4 \cdot 6} \frac{x^{7}}{7}+\cdots$

Fur $x=1$ wird are $\sin x=\frac{\pi}{2}$.

432) Die Reihe fur $\operatorname{arctg} x$. Setzt man:

$\operatorname{arctg} x=A+B x+C x^{2}+D x^{3}+E x^{4}+\cdots$,

so ist $A=0$ für $x=0$. Die Differentation giebt

$$
\frac{1}{1+x^{2}}=B+2 C x+3 D x^{2}+4 E x^{3}+\ldots
$$

Da nun weiter, wie bekannt

$$
\frac{1}{1+x^{2}}=1-x^{2}+x^{4}-x^{6}+\ldots
$$

so findet man durch Vergleichung, dass:

$$
\begin{gathered}
B=1, \quad C=0, \quad D=-\frac{1}{3}, \quad E=0, \text { usw. } \\
\operatorname{arctg} x=\frac{x}{1}-\frac{x^{3}}{3}+\frac{x^{5}}{5}-\frac{x^{7}}{7}+\cdots
\end{gathered}
$$

Für $x=1$ ist are $\operatorname{tg} x=\frac{\pi}{4}$, und man hat:

$$
\frac{\pi}{4}=1-\frac{1}{3}+\frac{1}{5}-\frac{1}{7}+\frac{1}{9}-\frac{1}{11}+\ldots
$$

Werden die Glieder paarweise vereinigt, so entsteht die zur Berechnung von $\pi$ praktischere Form:

$$
\frac{\pi}{4}=2\left(\frac{1}{1.3}+\frac{1}{5.7}+\frac{1}{9.11}+\ldots .\right) .
$$

Setzt $\operatorname{man} \operatorname{tg} \alpha=\frac{1}{2}$ und $\operatorname{tg} \beta=\frac{1}{3}$, so ist $\operatorname{tg}(\alpha+\beta)=1$ und daher $(\alpha+\beta)=\frac{\pi}{4}$. Man darf mithin setzen:

$$
\frac{\pi}{4}=\left(\frac{1}{2}+\frac{1}{3}\right)-\frac{1}{3}\left(\frac{1}{2^{3}}+\frac{1}{3^{3}}\right)+\frac{1}{5}\left(\frac{1}{2^{5}}+\frac{1}{3^{5}}\right)-\ldots
$$

In allen vorstehenden Aufgaben ist jedesmal die Restform der Maclaurinschen Reihe zu untersuchen. 
Zum Schlusse dieses Kapitels stellen wir noch einige später notwendige Formeln auf. Wird in der Reihe für $e^{x}$ an die Stelle von $x$ die imaginäre Variabele $i x$ gesetzt, so entsteht:

$$
\begin{aligned}
& e^{i x}=1-\frac{x^{2}}{1.2}+\frac{x^{4}}{1.2 .3 .4}-\frac{x^{6}}{1.2 \ldots .6}+\cdots \\
& \quad+i\left(\frac{x}{1}-\frac{x^{3}}{1.2 .3}+\frac{x^{5}}{1.2 .3 .4 .5}-\cdots\right),
\end{aligned}
$$

oder es ist $e^{i x}=\cos x+i \sin x$, und allgemein:

$$
e^{i n x}=\cos (n x)+i \sin (n x) .
$$

Da nun auch $e^{i n x}=(\cos x+i \sin x)^{n}$ ist, so erhalten wir leicht durch Vergleichung die Formel von Moivre wieder. Aus

folgt leicht:

$$
e^{i x}=\cos x+i \sin x \text { und } e^{-i x}=\cos x-i \sin x
$$

$$
\cos x=\frac{e^{i x}+e^{-i x}}{2}, \sin x=\frac{e^{i x}-e^{-i x}}{2 i},
$$

worin auch $n x$ statt $x$ gesetzt werden darf.

Tritt ebenso in Aufgabe No.424) an die Stelle von $x$ die imaginäre Variabele $i x$, so erhält man:

$$
\lg \frac{1+i x}{1-i x}=2 i\left(\frac{x}{1}-\frac{x^{3}}{3}+\frac{x^{5}}{5}-\ldots\right),
$$

woraus dann unter Berilcksichtigung der Reihe für are $\operatorname{tg} x$ folgt:

$$
\operatorname{arctg} x=\frac{1}{2 i} \lg \frac{1+i x}{1-i x}
$$




\section{Funktionen von zwei unabhängigen Variabelen.}

$\S 12$. Entwiekelung der Differentialquotienten.

Sind in der gegebenen Funktion $z=f(x, y)$ die beiden Variabelen $x$ und $y$ unabhängig von einander, so können die Werte, welche $z$ nach einander annimmt, wenn man den Variabelen $x$ und $y$ immer andere und andere Werte beilegt, auf folgende Weise graphisch veranschaulicht werden:

In der gewöhnlichen Koordinatenebene benutzt man in bekannter Weise das gewählte $x$ und $y$ zur Festlegung eines Punktes $D$ (Fig. 8), errichtet in

Fig. 11.

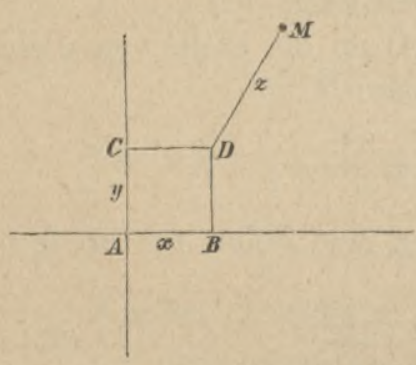

$D$ ein Perpendikel zur Koordinatenebene und trägt darauf den Wert von $z$ ab, welcher den gewählten Werten für $x$ und $y$ entspricht. So erhält man den Punkt $M$ im Raume. Bei mehrwertigen Funktionen findet man mehr als einen Punkt auf der nämlichen Senkrechten. Der Inbegriff aller Lagen, welohe der Punkt $M$ vermöge der Gleichung $z=f(x, y)$ annehmen kann, ist eine Oberfläche.

Eine Änderung des Funktionswertes $z=f(x, y)$ kann nun auf dreierlei Weise veranlasst werden, und zwar:

1) dadurch, dass bei festem $y$ nur $x$ ubergeht in $x_{1}$,

2) dadurch, dass bei festem $x$ nur $y$ übergeht in $y_{1}$,

3) dadurch, dass gleichzeitig $x$ und $y$ ïbergehen in $x_{1}$ und $y_{1}$. Demgemäss müssen auch 3 versohiedene Arten von Differenzen der Funktionswerte unterschieden werden, nämlich:

1) $f\left(x_{1}, y\right)-f(x, y)$; 2) $f\left(x, y_{1}\right)-f(x, y)$; 3) $f\left(x_{1}, y_{1}\right)-f(x, y)$. 
Dass man die Werte:

$$
\frac{\partial f}{\partial x}=\lim \frac{f\left(x_{1}, y\right)-f(x, y)}{x_{1}-x}, \quad \frac{\partial f}{\partial y}=\lim \frac{f\left(x, y_{1}\right)-f(x, y)}{y_{1}-y}
$$

die partiellen Differentialquotienten der Funktion nennt, wurde Pg. 15 und 17 erörtert, wo sich auch der Modus ihrer Ableitung angegeben findet. Wir haben uns deshalb hier nur noch mit derjenigen Veränderung $d z$ zu befassen, welche der Funktionswert $z$ dadurch erfährt, dass sich die beiden Variabelen $x$ und $y$ gleichzeitig um die verschwindend kleinen Werte $d x$ und $d y$ ändern. Wenn den beliebig, aber doch ganz bestimmt gewählten Werten $x, y$ und $x_{1}, y_{1}$ die Funktionswerte $z$ und $z_{1}$, zugehören, so ist $\left(z_{1}-z\right)=f\left(x_{1}, y_{1}\right)-f(x, y)$, oder:

$z_{1}-z=\frac{f\left(x_{1}, y_{1}\right)-f\left(x, y_{1}\right)}{x_{1}-x}\left(x_{1}-x\right)+\frac{f\left(x, y_{1}\right)-f(x, y)}{y_{1}-y}\left(y_{1}-y\right)$.

Durch den Übergang zu den Grenzen wird dieser Ausdruck in den folgenden übergeführt:

$$
d z=\frac{\partial f}{\partial x} d x+\frac{\partial f}{\partial y} d y
$$

Wird diese Formel nach ihrer eigenen Anleitung nochmals differentiiert und dabei berücksichtigt, dass $d x$ und $d y$ konstante Werte sind, so entsteht:

$$
\begin{aligned}
& d^{2} z=\frac{\partial\left(\frac{\partial f}{\partial x} d x+\frac{\partial f}{\partial y} d y\right)}{\partial x} d x+\frac{\partial\left(\frac{\partial f}{\partial x} d x+\frac{\partial f}{\partial y} d y\right)}{\partial y} d y \\
& d^{2} z=\frac{\partial^{2} f}{\partial x^{2}} d x^{2}+2 \frac{\partial^{2} f}{\partial x \partial y} d x d y+\frac{\partial^{2} f}{\partial y^{2}} d y^{2}
\end{aligned}
$$

\section{Aufgaben.}

433) $z=3 x^{2}+x y^{2}+y^{4}$

434) $z=\frac{x y}{x+y}$

435) $z=\sqrt{x^{2}-y^{2}}$

436) $z=\left(2 x-5 y^{2}\right)^{3}$

$$
\begin{aligned}
d z & =\left(6 x+y^{2}\right) d x+\left(2 x y+4 y^{3}\right) d y \\
& =\frac{x^{2} d y+y^{2} d x}{(x+y)^{2}} \\
" & =\frac{x d x-y d y}{\sqrt{x^{2}-y^{2}}} \\
& =3\left(2 x-5 y^{2}\right)^{2}(2 d x-10 y d y
\end{aligned}
$$




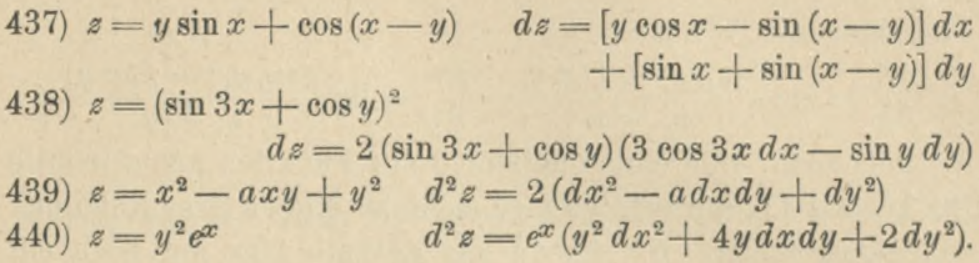

§ 13. Maxima und Minima von Funktionen mit zwei unabhängigen Variabelen.

Wenn auf der eingangs des vorhergehenden Paragraphen erwähnten Funktionsoberfläche der Punkt $M$ von der $X Y$-Ebene einen Abstand $z$ hat, der algebraisch grösser ist als furr alle Punkte seiner unmittelbarsten Umgebung, so nennt man ihn einen Maximalpunkt; ist er dagegen kleiner, so heisst er ein Minimalpunkt. Der zugehörige Funktionswert $z$ wird dann ein Maximum, beziehungsweise ein Minimum genannt. Werden dureh einen solchen Punkt $M$ nach beliebigen Richtungen Ebenen senkrecht zur $X Y$-Ebene gelegt, so entstehen auf der Oberfläche Schnittkurven, und es mutssen alle Tangenten, welche durch $M$ an diese Kurven gelegt werden können, parallel zur $X Y$-Ebene sein. Legen wir in der $X Y$-Ebene beliebige Kurven durch denPunkt $D$, so entsprechen diesen ebenfalls beliebige Gleichungen zwischen den Variabelen $x$ und $y$. Jedem Punkte einer solchen Kurve entspricht ein bestimmtes Wertpaar $x$ und $y$, und diesem wieder ein ganz bestimmtes $z$, woraus dann folgt, dass jeder Kurve in der $X Y$-Ebene eine ganz bestimmte Kurve auf der Funktionsoberfläche entsprechen muss. Kann nun ein Wert $z$ so gefunden werden, dass er für alle diese Funktionskurven ein Maximum oder ein Minimum vorstellt, so ist er auch ein Maximum oder ein Minimum der Funktion. In $\S 10$ haben wir Werte für $x$ und $y$ ermittelt, für welche $z=f(x, y)$ ebenfalls ein Maximal- oder ein Minimalwert wurde, doch mussten die gefundenen Werte noch weiter der Bedingung genügen: $\varphi(x, y)=0$. Daher unterseheidet sich unsere jetzige Aufgabe von der damaligen nur durch den Umstand, dass die erwähnte Bedingung 
ganz fehlt, d. h. die Abhängigkeit zwischen $x$ und $y$ beliebig gewählt werden darf. Fügen wir deshalb der gegebenen Funktion $z=f(x, y)$ noch die willkürliche Bedingung $\varphi(x, y)=0$, oder statt ihrer noch die beiden willkürlichen Bedingungen $x=\chi(t)$, $y=\psi(t)$ hinzu, so ist diese Aufgabe auf die fruhere zurtickgefuihrt. Hierdurch ist $z$ in eine Funktion der einzigen Variabelen $t$ umgewandelt, und es muss für den Fall eines Maximums oder Minimums:

$$
\frac{d z}{d t}=\frac{\partial f}{\partial x} \cdot \frac{d x}{d t}+\frac{\partial f}{\partial y} \cdot \frac{d y}{d t}=0
$$

sein. Bei der Willkürlichkeit von $\frac{d x}{d t}=x^{\prime}$ und $\frac{d y}{d t}=y^{\prime}$ kann diese Bedingung nur erfullt werden, wenn:

$$
\frac{\partial f}{\partial x}=0 \text { und } \frac{\partial f}{\partial y}=0 .
$$

Aus diesen Gleichungen gehen diejenigen Werte von $x$ und $y$ hervor, welche $z$ zu einem Maximum oder Minimum machen. Diese selbst werden, wie gewöhnlich, durch das Vorzeichen des zweiten Differentialquotienten von einander unterschieden. Es ist: $\frac{d^{2} z}{d t^{2}}=\frac{\partial f}{\partial x} x^{\prime \prime}+\frac{\partial f}{\partial y} y^{\prime \prime}+x^{\prime}\left(\frac{\partial^{2} f}{\partial x^{2}} x^{\prime}+\frac{\partial^{2} f}{\partial x \partial y} y^{\prime}\right)$

Gemäss der Relationen (37) wird:

$$
+y^{\prime}\left(\frac{\partial^{2} f}{\partial x \partial y} x^{\prime}+\frac{\partial^{2} f}{\partial y^{2}} y^{\prime}\right)
$$

$$
\frac{d^{2} z}{d t^{2}}=\frac{\partial^{2} f}{\partial x^{2}} x^{\prime 2}+2 \frac{\partial^{2} f}{\partial x \partial y} x^{\prime} y^{\prime}+\frac{\partial^{2} f}{\partial y^{2}} y^{\prime 2} .
$$

Die zweiten Differentialquotienten sind hier konstante Grössen, weil sie mit den aus (37) hervorgehenden Werten von $x$ und $y$ zu bilden sind. Wir nennen sie $a, b$ und $c$ und haben:

$\frac{d^{2} z}{d t^{2}}=a x^{\prime 2}+2 b x^{\prime} y^{\prime}+c y^{\prime 2}=\frac{\left(a x^{\prime}+b y^{\prime}\right)^{2}+\left(a c-b^{2}\right) y^{\prime 2}}{a}$.

Der letzte Ausdruck bleibt für alle Werte von $x^{\prime}$ und $y^{\prime}$ positiv, wenn $\left(a c-b^{2}\right)>0$ und zugleich $a>0$ ist. Die Funktion hat dann ein Minimum. Ist dagegen $\left(a c-b^{2}\right)>0$ und $a<0$, so 
muss der Ausdruck stets negativ werden, wie man $x^{\prime}$ und $y^{\prime}$ auch wählen mag. Die Funktion hat ein Maximum. Ist endlich $\left(a c-b^{2}\right)<0$, so kann der Wert durch geeignete Wahl von $x^{\prime}$ und $y^{\prime}$ nach Belieben positiv oder negativ gemacht werden. Hiernach ist es für Maximal- oder Minimalwerte ein gemeinsames Erfordernis, dass $\frac{\partial^{2} f}{\partial x^{2}} \cdot \frac{\partial^{2} f}{\partial y^{2}}-\left(\frac{\partial^{2} f}{\partial x \partial y}\right)^{2}>0$ ist, und es ist dann

$$
\begin{aligned}
& z \text { ein Maximum, wenn } \frac{\partial^{2} f}{\partial x^{2}}<0, \\
& z \text { ein Minimum, wenn } \frac{\partial^{2} f}{\partial x^{2}}>0 .
\end{aligned}
$$

\section{A $\mathrm{u} f \mathrm{gab}$ en.}

441) $z=x^{2}+y^{2}+x y-6 x-4 y+5$ Min. für $x=\frac{8}{3}, y=\frac{2}{3}$

442) $z=x^{2}-6 x y+y^{3}+3 x+6 y \quad$ " $\quad x=\frac{27}{2}, y=5$

443) $z=4 x y+\frac{1}{x}+\frac{1}{y}$

",$x=y=\frac{1}{2} \sqrt[3]{2}$

444) $z=(x+y)^{2}-(x+5 y+x y)$

" $, x=-1, y=3$

445) $z=x y^{2}(a-x-y)^{3}$

Max. für $x=\frac{a}{6}, y=\frac{a}{3}$

446) $z=\sin x+\sin y+\sin (x+y)$

$$
" \quad n x=y=60^{\circ}
$$

447) $z=\sin x \sin y \sin (\alpha-x-y)$

त $\quad x=y=\frac{\alpha}{3}$

448) $z=x^{3}-3 a x y+y^{3}$

$\eta \quad n x=y=a$.

449) DasVolumen eines rechtwinkeligen Parallelopipedesist $=a^{3}$. Wie gross müssen die Kanten sein, wenn die Oberfläche ein Min. werden soll?

Der Körper ist ein Würfel, dessen Kanten $=a$ sind.

450) Eine Zahl $a$ soll in 3 solche Teile zerlegt werden, dass deren Produkt ein Max. wird.

$$
z=x y(a-x-y) \text { wird Max., wenn jeder Teil }=\frac{a}{3} \text { ist. }
$$

451) In einen Kreis soll das an Fläche grösste Dreieck gezeichnet werden. 
Die zu den Seiten gehörenden Centriwinkel mögen $x, y$ und $(2 \pi-x-y)$ heissen, so wird $F=\frac{1}{2} r^{2}[\sin x+\sin y$ $+\sin (2 \pi-x-y)]$ ein Max., wenn die 3 Centriwinkel gleich, mithin auch die Dreieckseiten gleich sind.

452) Die Summe der 3 Seiten eines Dreiecks ist $=2 s$. Wie muss man die einzelnen Seiten wählen, wenn die Fläche ein Max. sein soll?

$F=\sqrt{s(s-x)(s-y)(x+y-s)}$ wird ein Max., wenn die 3 Seiten gleich sind.

453) Aus einer Kugel soll das an Volumen grösste rechtwinkelige Parellelopiped geschnitten werden.

Kugelhalbmesser $=\boldsymbol{r}$, die halben Kanten $x, y, u$, so besteht die Relation: $x^{2}+y^{2}+u^{2}=r^{2}$. Das Volumen $z=8 x y u$ wird Max. für $x=y=u=r \sqrt{\frac{1}{3}}$.

\section{14. Homogene Funktionen.}

Wir sagen, der Ausdruck $a x^{2} y^{3}$ sei von der fünften Dimension, weil die Exponentensumme der beiden Variabelen 5 beträgt, und nennen $\frac{a\left(x^{4}+y^{2} x^{2}\right)}{x+y}$ einen Ausdruck dritter Dimension, weil die Differenz aus der Exponentensumme des Zählers und des Nenners gleich 3 ist. Dass die Dimension eines Ausdrucks auch eine negative oder gebrochene Zahl sein kann, ist selbstverständlich. Sind die einzelnen Teile einer Funktion von gleich hoher Dimension, so heisst die Funktion homogen. Zur sicheren Beurteilung, ob eine Funktion die erwähnte Eigenschaft besitze, dient das folgende Kriterium: Ersetzt man die Variabelen $x, y, z$, usw. durch die Werte $t x, t y, t z$, usw., wobei $t$ einen beliebigen Faktor vorstellt, so muss man für eine Funktion von $n^{\text {ter }}$ Dimension die Relation finden:

$$
f(t x, t y, t z, . .)=t^{n} f(x, y, z, . .) \text {. }
$$

1. Beispiel: $f(x, y)=5 x^{2}-2 x y+7 y^{2}$;

$$
f(t x, t y)=5 t^{2} x^{2}-2 t x t y+7 t^{2} y^{2}=t^{2} f(x, y) \text {. }
$$


2. Beispiel: $f(x, y, z)=\frac{x^{3} z^{\frac{3}{2}}-y^{4} x^{\frac{1}{2}}+y^{\frac{5}{2}} z^{2}}{x^{\frac{1}{2}} y^{\frac{1}{2}}+z}$

$f(t x, t y, t z)=\frac{t^{3} x^{3} t^{\frac{3}{2}} z^{\frac{3}{2}}-t^{4} y^{4} t^{\frac{1}{2}} x^{\frac{1}{2}}+t^{\frac{5}{2}} y^{\frac{5}{2}} t^{2} z^{2}}{t^{\frac{1}{2}} x^{\frac{1}{2}} t^{\frac{1}{2}} y^{\frac{1}{2}}+t z}=t^{\frac{7}{2}} f(x, y, z)$.

Euler's Lehrsatz über homogene Funktionen. Wir setzen in (38) $t x=u, t y=v, t z=w$, und differentiieren die beiden Seiten nach $t$ :

$$
\frac{\partial f}{\partial u} \cdot \frac{d u}{d t}+\frac{\partial f}{\partial v} \cdot \frac{d v}{d t}+\frac{\partial f}{\partial w} \cdot \frac{d w}{d t}=n t^{n-1} f(x, y, z)
$$

oder, weil $\frac{d u}{d t}=x, \frac{d v}{d t}=y, \frac{d w}{d t}=z$ ist,

$$
\frac{\partial f}{\partial u} \cdot x+\frac{\partial f}{\partial v} \cdot y+\frac{\partial f}{\partial w} \cdot z=n t^{n-1} f(x, y, z) .
$$

Lässt man hierin die willkürliche Grösse $t$ in 1 übergehen, so verwandelt sich $\frac{\partial f}{\partial u}$ in $\frac{\partial f}{\partial x}, \frac{\partial f}{\partial v}$ in $\frac{\partial f}{\partial y}, \frac{\partial f}{\partial w}$ in $\frac{\partial f}{\partial z}$ und man erhält:

$$
x \cdot \frac{\partial f}{\partial x}+y \cdot \frac{\partial f}{\partial y}+z \cdot \frac{\partial f}{\partial z}=n \cdot f(x, y, z) .
$$

Diese Formel stellt den Satz von Euler dar.

1. Beispiel: $f=\frac{x^{2}}{a^{2}}+\frac{y^{2}}{b^{2}}-z^{2}$;

$$
\frac{2 x}{a^{2}} \cdot x+\frac{2 y}{b^{2}} \cdot y-2 z \cdot z=2\left(\frac{x^{2}}{a^{2}}+\frac{y^{2}}{b^{2}}-z^{2}\right) .
$$

2. Beispiel: $f=x^{3}+2 a x^{2} y-a y^{3}=0$;

$\left(3 x^{2}+4 a x y\right) x+\left(2 a x^{2}-3 a y^{2}\right) y=3\left(x^{3}+2 a x^{2} y-a y^{3}\right)$.

3. Beispiel: $f=\sqrt{x^{3}-y^{2} z}=R$;

$$
\begin{gathered}
\frac{\partial f}{\partial x}=\frac{3 x^{2}}{2 R}, \frac{\partial f}{\partial y}=\frac{-2 y z}{2 R}, \frac{\partial f}{\partial z}=\frac{-y^{2}}{2 R} \\
\frac{3 x^{3}-2 y^{2} z-y^{2} z}{2 R}=\frac{3}{2}\left(\frac{x^{3}-y^{2} z}{R}\right)=\frac{3}{2} \sqrt{x^{3}-y^{2} z} .
\end{gathered}
$$

4. Beispiel: $f=\frac{x y}{x^{3}+y^{3}}$;

$$
x \cdot \frac{\partial f}{\partial x}+y \cdot \frac{\partial f}{\partial y}=-\frac{y^{4} x+x^{4} y}{\left(x^{3}+y^{8}\right)^{2}}=-\frac{x y}{x^{8}+y^{3}} .
$$


5. Beispiel: $f=\frac{1}{\sqrt{x}}+\frac{\sqrt{y}}{z}$;

$$
-\frac{1}{2} x^{-\frac{3}{2}} \cdot x+\frac{1}{2} \frac{y^{-\frac{1}{2}}}{z} \cdot y-\frac{y^{\frac{1}{2}}}{z^{2}} \cdot z=-\frac{1}{2}\left(\frac{1}{\sqrt{x}}+\frac{\sqrt{y}}{z}\right) .
$$

§ 15. Die Reihen von Taylor und Maclaurin für Funktionen mit zwel unabhängigen Variabelen.

Ersetzen wir in $f(x, y)$ die Variabele $x$ durch $x+\xi$ und $y$ durch $y+\eta$, so kann die Funktion in eine Reihe nach Potenzen und Produkten von $\xi$ und $\eta$ entwickelt werden. Um diese Entwickelung auf $\S 11$ zurückzufuhren, setzen wir $\xi=r t, \eta=s t$ und $f(x+r t, y+s t)=F(t)$, indem wir uns unter $x, y, r, s$ bestimmt gewählte Werte und unter $t$ eine Variabele vorstellen. Nach dem Maclaurin'schen Satze ist jetzt:

$$
F(t)=F(0)+F^{\prime}(0) \frac{t}{1}+F^{\prime \prime}(0) \frac{t^{2}}{1.2}+F^{\prime \prime \prime}(0) \frac{t^{3}}{1.2 .3}+\ldots
$$

Bei der Bildung der Funktionen $F^{\prime}, F^{\prime \prime}$, usw. ist es zweckmässig, $x+r t=u, y+s t=v$ zu setzen, wodurch $F(t)$ in $f(u, v)$ umgewandelt wird. Zugleich ist $\frac{d u}{d t}=r, \frac{d v}{d t}=s$, und die höheren Differentialquotienten versohwinden. So ist:

$$
\begin{aligned}
F(t) & =f(u, v) \\
F^{\prime}(t) & =\frac{\partial f}{\partial u} \cdot \frac{d u}{d t}+\frac{\partial f}{\partial v} \cdot \frac{d v}{d t} \\
F^{\prime \prime}(t) & =\frac{\partial^{2} f}{\partial u^{2}}\left(\frac{d u}{d t}\right)^{2}+2 \frac{\partial^{2} f}{\partial u \partial v} \frac{d u}{d t} \frac{d v}{d t}+\frac{\partial^{2} f}{\partial v^{2}}\left(\frac{d v}{d t}\right)^{2} \\
F^{\prime \prime \prime}(t) & =\frac{\partial^{3} f}{\partial u^{8}}\left(\frac{d u}{d t}\right)^{3}+3 \frac{\partial^{3} f}{\partial u^{2} \partial v}\left(\frac{d u}{d t}\right)^{2} \frac{d v}{d t}+3 \frac{\partial^{3} f}{\partial u \partial v^{2}} \frac{d u}{d t}\left(\frac{d v}{d t}\right)^{2} \\
& +\frac{\partial^{3} f}{\partial v^{8}}\left(\frac{d v}{d t}\right)^{3} ; \text { usw. }
\end{aligned}
$$

Setzt man $t=0$, so geht $f(u, v)$ in $f(x, y)$ über und ebenso verwandeln sich die partiellen Differentialquotienten von $f$ nach $u$ und $v$ in die Differentialquotienten nach $x$ und $y$. So entstehen die Werte $F(0), F^{\prime}(0), F^{\prime \prime}(0)$ usw. und es giebt: 


$$
\begin{gathered}
F(t)=f(x, y)+\left[\frac{\partial f}{\partial x} r+\frac{\partial f}{\partial y} s\right] \frac{t}{1}+\left[\frac{\partial^{2} f}{\partial x^{2}} r^{2}+2 \frac{\partial^{2} f}{\partial x \partial y} r s+\frac{\partial^{2} f}{\partial y^{2}} s^{2}\right] \frac{t^{2}}{1.2} \\
+\left[\frac{\partial^{3} f}{\partial x^{3}} r^{3}+3 \frac{\partial^{3} f}{\partial x^{2} \partial y} r^{2} s+3 \frac{\partial^{3} f}{\partial x \partial y^{2}} r s^{2}+\frac{\partial^{3} f}{\partial y^{3}} s^{3}\right] \frac{t^{3}}{1.2 .3} \cdots
\end{gathered}
$$

Werden nun für $F(t), r t$, st die Werte wieder gesetzt, die sie vertreten, so ergiebt sich:

$$
\begin{gathered}
f(x+\xi, y+\eta)=f(x, y)+\left[\frac{\partial f}{\partial x} \xi+\frac{\partial f}{\partial y}\right]+\left[\frac{\partial^{2} f}{\partial x^{2}} \frac{\xi^{2}}{1.2}\right. \\
\left.+2 \frac{\partial^{2} f}{\partial x \partial y} \frac{\xi \eta}{1.2}+\frac{\partial^{2} f}{\partial y^{2}} \frac{\eta^{2}}{1.2}\right]+\left[\frac{\partial^{3} f}{\partial x^{3}} \frac{\xi^{3}}{1.2 .3}\right.
\end{gathered}
$$

$\left.+3 \frac{\partial^{3} f}{\partial x^{2} \partial y} \frac{\xi^{2} \eta}{1.2 .3}+3 \frac{\partial^{3} f}{\partial x \partial y^{2}} \frac{\xi \eta^{2}}{1.2 .3}+\frac{\partial^{3} f}{\partial y^{3}} \frac{\eta^{3}}{1.2 .3}\right]+\cdots$

Wird darin $x=0$ und $y=0$ gesetzt, so erhält man die Reihe für $f(\xi, \eta)$, welche in die Reihe für $f(x, y)$ übergeht, wenn $\xi$ mit $x$ und $\eta$ mit $y$ vertauseht wird.

\section{A u f g a ben.}

454) Wenn $f(x, y)=x^{3}+x y^{2}$ ist, so soll $f(x+\xi, y+\eta)$ allgemein entwickelt und dann $\xi=1, \eta=2$ gesetzt werden. $f(x+1, y+2)=\left[x^{3}+y^{2} x\right]+\left[3 x^{2}+y^{2}+4 x y\right]+[7 x+4 y]+5$.

455) Gegeben ist $f(x, y)=\sin x \cos y$. Es soll $f(x+\delta, y+\delta)$ in eine Reihe entwickelt werden.

$f(x+\delta, y+\delta)=\sin x \cos y+\cos (x+y) \frac{\delta}{1}$

$-2 \sin (x+y) \frac{\delta^{2}}{1.2}-4 \cos (x+y) \frac{\delta^{3}}{1.2 .3} \cdots$

456) Es soll die Funktion $a^{x} \lg (1+y)$ in eine Reihe entwickelt werden.

Man lässt $x$ in $(x+\xi), y$ in $(y+\eta)$ tibergehen, entwickelt die Funktionen nach Potenzen und Produkten von $\xi$ und $\eta$, setzt dann in den als Koefficienten auftretenden Differentialquotienten $x=0$ und $y=0$ und vertauscht hierauf $\xi$ mit $x$ und $\eta$ mit $y$. So erbält man: $a^{x} \lg (1+y)=y+x y \lg a-\frac{1}{2} y^{2}+\frac{x^{2} y}{2}(\lg a)^{2}-\frac{x y^{2}}{2} \lg a+\frac{1}{3} y^{3}+\ldots$ 


\section{Integralrechnung.}

\section{Unbestimmte Integrale.}

\section{§ 1. Die einfachen Integralformen.}

Die Integralrechnung hat zunächst die Aufgabe zu lösen, zu einem gegebenen Differentialquotienten die ursprïngliche Funktion wieder herzustellen, oder allgemeiner, zu einer gegebenen Funktion $f(x)$ diejenige Funktion $F(x)$ zu finden, deren Differentialquotient $f(x)$ ist. Wir nennen dann $F$ das Integral von $f$ und sagen, es sei

$$
\int f(x) d x=F(x), \text { wenn } \frac{d[F(x)]}{d x}=f(x)
$$

ist; $f(x)$ heisse der Integrand. Da nun der Differentialquotient von $F(x)$ unverändert bleibt, wenn zu $F(x)$ eine beliebige Konstante $C$ zugefugt wird, so sind $\mathrm{zu}$ einem vorgelegten Integranden unzählig viele Integrale möglich. Doch können ihre Werte nur um eine additive Konstante verschieden sein. Denn wären $F$ und $G$ zwei Integrale von $f$, so wäre

$$
f=\frac{d F}{d x}=\frac{d G}{d x} ; \quad \frac{d(F-G)}{d x}=0,
$$

und also nach S. $65 F-G$ eine Konstante.

Aus unseren Definitionen geht hervor, dass

$$
\int d x=x+C, \quad \int d[f(x)]=f(x)+C .
$$

Fine Anzahl einfacher Integralformen wird erhalten, wenn man die in $\S 1$ zusammengestellten Differentialformen kurzweg umkehrt und schreibt: 
1) $\int a d x=a x+C$

2) $\int a x^{n} d x=\frac{a x^{n+1}}{n+1}+C$

3) $\int \frac{1}{x} d x=\lg x+C$

4) $\int e^{x} d x=e^{x}+C$

5) $\int a^{x} d x=\frac{a^{x}}{\lg a}+C$

6) $\int \sin x d x=-\cos x+C$

7) $\int \cos x d x=\sin x+C$

8) $\int \frac{1}{\cos ^{2} x} d x=\operatorname{tg} x+C$

9) $\int \frac{1}{\sin ^{2} x} d x=-\operatorname{cotg} x+C$

10) $\int \frac{1}{1+x^{2}} d x=\operatorname{arctg} x+C=-\operatorname{arccotg} x+C_{1}$

11) $\int \frac{1}{\sqrt{1-x^{2}}} d x=\arcsin x+C=-\arccos x+C_{1}$.

Sind $u$ und $v$ Funktionen von $x$, so ist $\frac{d(u+v)}{d x}=u^{\prime}+v^{\prime}$, und daher ist umgekehrt:

$$
\int\left(u^{\prime}+v^{\prime}\right) d x=u+v=\int u^{\prime} d x+\int v^{\prime} d x
$$

das zeigt: Eine Summe aus einer endlichen Zahl von Summanden integriert man, indem man jeden Teil integriert.

Ist $a$ konstant, so ist $d(a \cdot u)=a \cdot u^{\prime}$ und darum auch:

$$
\int a \cdot u^{\prime} d x=a \cdot u=a \int u^{\prime} d x .
$$

Ein konstanter Faktor unter dem Integralzeichen darf vor dasselbe gestellt werden. 
Aufgaben.

Die beliebige Konstante ist den Lösungen nicht zugefugt.

12) $\int 7 x^{4} d x=\frac{7 x^{5}}{5}$

13) $\int \frac{2}{3 x^{5}} d x=-\frac{1}{6 x^{4}}$

14) $\int \sqrt{x} d x=\frac{2}{3} \sqrt{x^{3}}$

15) $\int \frac{1}{\sqrt{x}} d x=2 \sqrt{x}$

16) $\int 5 \sqrt[3]{x^{2}} d x=3 \sqrt[3]{x^{5}}$

17) $\int \frac{a}{\sqrt[4]{5 x^{8}}} d x=4 a \sqrt[4]{\frac{x}{5}}$

18) $\int(a+b x) d x=a x+\frac{b x^{2}}{2}$, oder $=\frac{(a+b x)^{2}}{2 b}$, indem sich beide Werte nur durch eine Konstante unterscheiden.

19) $\int\left(5 x^{2}-6 x+3-\frac{2}{x}+\frac{5}{x^{2}}\right) d x=\frac{5 x^{3}}{3}-3 x^{2}+3 x-2 \lg x-\frac{5}{x}$ 20) $\int \frac{\left(x^{2}-1\right)^{3}}{x} d x=\frac{x^{6}}{6}-\frac{3 x^{4}}{4}+\frac{3 x^{2}}{2}-\lg x$

21) $\int(3+2 \sqrt[4]{x})^{3} d x=27 x+\frac{216}{5} x^{\frac{5}{4}}+24 x^{\frac{3}{2}}+\frac{32}{7} x^{\frac{7}{4}}$

22) $\int \frac{\sqrt{x}-2 \sqrt[3]{x^{2}}+4 \sqrt[4]{5 x^{8}}}{6 \sqrt[3]{x}} d x=\frac{1}{7} x^{\frac{7}{6}}-\frac{1}{4} x^{4}+\frac{8}{17} \sqrt[12]{125 x^{17}}$

23) $\int \frac{3+5 \sqrt[3]{x^{2}}}{\sqrt{x^{3}}} d x=-\frac{6}{\sqrt{x}}+30 \sqrt[6]{x}$

Die Methode der Substitution. Sieht man, dass die Funktion unter dem Integralzeichen ein sogenanntes vollständiges Differential, d. h. der Differentialquotient einer einfachen und bekannten Funktion multipliziert mit $d x$ ist, so kann das Integral sofort hingeschrieben werden. Gewöhnlich muss aber diese Form, wenn sie uberhaupt möglich ist, erst durch Transformation herbeigeführt werden, indem man $x=\varphi(z)$ 
setzt und den gegebenen Differentialquotienten nach $x$ in den Differentialquotienten der nämlichen Funktion, aber genommen nach $z$, umwandelt. Es muss dies auf folgende Weise geschehen: Bei dem Integral $y=\int f(x) d x$ ist $\frac{d y}{d x}=f(x)$. Wird nun $x=\varphi(z)$ gesetzt, so dass $y$ als Funktion von $z$ erscheint, dann ist $\frac{d y}{d z}=\frac{d y}{d x} \cdot \frac{d x}{d z}=f[\varphi(z)] \cdot \varphi^{\prime}(z)$. Hieraus folgt:

$$
y=\int f(x) d x=\int f[\varphi(z)] \cdot \varphi^{\prime}(z) d z .
$$

Man muss nun versuchen, $\varphi$ so zu wählen, dass der Integrand des letzten Integrals möglichst einfach wird.

1. Beispiel: $y=\int \frac{d x}{e^{x}+e^{-x}} ; \quad e^{x}=z$ oder $x=\lg z$; $y=\int \frac{1}{z+z^{-1}} \cdot \frac{d z}{z}=\int \frac{d z}{z^{2}+1} ; \quad y=\operatorname{arctg} z=\operatorname{arctg} e^{x}$.

2. Beispiel: $y=\int \frac{x d x}{\sqrt{x^{2}+a^{2}}} ; \sqrt{x^{2}+a^{2}}=z$ od. $x=\sqrt{z^{2}-a^{2}}$ $y=\int \frac{\sqrt{z^{2}-a^{2}}}{z} \frac{z}{\sqrt{z^{2}-a^{2}}} d z=\int d z=z=\sqrt{x^{2}+a^{2}}$.

3. Beispiel: $y=\int \frac{\operatorname{arctg}^{2} x}{1+x^{2}} d x$; aretg $x=z$ oder $x=\operatorname{tg} z$ $y=\int \frac{z^{2}}{1+\operatorname{tg}^{2} z} \frac{d z}{\cos ^{2} z}=\int z^{2} d z=\frac{1}{3} z^{3}=\frac{1}{3} \operatorname{arctg}^{3} x$.

Allgemeine Regeln über die Anwendung dieser Methode der Substitution giebt es nicht. Die Anwendung der Formel (1) kann an den folgenden Aufgaben geübt werden.

24) $y=\int(a+b x)^{n} d x ; \quad a+b x=z, \frac{d x}{d z}=\frac{1}{b}$;

$$
y=\frac{1}{b} \int z^{n} d z=\frac{1}{b} \frac{z^{n+1}}{n+1}=\frac{(a+b x)^{n+1}}{(n+1) b}
$$


25) $y=\int \frac{1}{a+b x} d x ; \quad a+b x=z$;

$$
y=\frac{1}{b} \int \frac{1}{z} d z=\frac{1}{b} \lg z=\frac{1}{b} \lg (a+b x)
$$

26) $y=\int \sqrt{a+b x} d x ; \quad a+b x=z^{2}, \quad \frac{d x}{d z}=\frac{2 z}{b}$;

$$
y=\frac{2}{b} \int z^{2} d z=\frac{2}{b} \cdot \frac{z^{3}}{3}=\frac{2}{3 b} \sqrt{(a+b x)^{3}}
$$

27) $y=\int e^{m x} d x ; \quad m x=z, \frac{d x}{d z}=\frac{1}{m}$;

$$
y=\frac{1}{m} \int e^{z} d z=\frac{e^{z}}{m}=\frac{e^{m x}}{m}
$$

28) $y=\int \frac{1}{a^{2}+x^{2}} d x ; x=a z$. Statt nun zu setzen: $\frac{d x}{d z}=a$, ist es gebräuchlich zu schreiben: $d x=a d z$;

$$
y=\frac{1}{a} \int \frac{1}{1+z^{2}} d z=\frac{1}{a} \operatorname{arctg} z=\frac{1}{a} \operatorname{arctg} \frac{x}{a}
$$

29) $y=\int \sin a x d x ; \quad a x=z, \quad d x=\frac{1}{a} d z$;

$$
y=\frac{1}{a} \int \sin z d z=-\frac{1}{a} \cos z=-\frac{1}{a} \cos a x
$$

$$
\text { 30) } \begin{aligned}
y & =\int \frac{1}{x^{2}+4 x+5} d x=\int \frac{1}{(x+2)^{2}+1} d x \\
y & =\int \frac{1}{z^{2}+1} d z=\operatorname{arctg} z=\operatorname{arctg}(x+2)
\end{aligned}
$$

Die Integration nach Teilen. Sind $u$ und $v$ Funktionen von $x$, so ist $\frac{d(u \cdot v)}{d x}=v \cdot u^{\prime}+u \cdot v^{\prime}$, und daraus folgt sofort: $\int v \cdot u^{\prime} d x+\int u \cdot v^{\prime} d x=u \cdot v$, wofür man auch setzen kann:

$$
\int u \cdot v^{\prime} d x=u \cdot v-\int v \cdot u^{\prime} d x .
$$

Diese Formel hat folgenden Sinn: Soll ein Produkt $u \cdot v^{\prime}$, dessen zweiter Faktor $v^{\prime}$ ein vollständiger Differentialquotient ist, integriert werden, so integriert man zunächst nur diesen Faktor $v^{\prime}$ und bildet aus dem Integral $v$ und dem unveränderten 
ersten Faktor $u$ das Produkt $u . v$; dann wird $u$ differentiiert, das Produkt $u^{\prime} \cdot v$ gebildet und $\int v \cdot u^{\prime} d x$ von $u . v$ abgezogen. Durch dieses Verfahren wird nur ein Teil des ganzen Integrales fertig erhalten, während der andere Teil sich noch unter dem Integralzeichen befindet. Ist dieses ubrig gebliebene Integral $\int v \cdot u^{\prime} d x$ aber einfacher als das gegebene, so ist die Methode zweckmässig; besonders ist sie dies dann, wenn durch wiederholte Anwendung das zurïckbleibende Integral immer einfacher und schliesslich auch in ausfuhrbarer Form erhalten wird. Da ein gegebener Integrand sich auf ganz verschiedene Arten in ein Produkt $u \cdot v^{\prime}$ zerlegen lässt, so ist die vorteilhafteste Wahl der Faktoren eine Sache der Gesohicklichkeit.

Wir lassen zunächst $\mathrm{zu}(2)$ einige Beispiele folgen.

31) $y=\int x^{3} e^{x} d x ; \quad u=x^{3}, \quad v^{\prime}=e^{x}$

$\int x^{3} e^{x} d x=x^{3} e^{x}-3 \int x^{2} e^{x} d x ; \int x^{2} e^{x} d x=x^{2} e^{x}-2 \int x e^{x} d x ;$ $\int x e^{x} d x=x e^{x}-\int e^{x} d x=x e^{x}-e^{x}$. Stellen wir nun noch das Ganze zusammen und addieren

$$
\begin{array}{rlrl}
\int x^{3} e^{x} d x & = & x^{3} e^{x}- & 3 \int x^{2} e^{x} d x \\
-3 \int x^{2} e^{x} d x & = & -3 x^{2} e^{x}+2.3 \int x e^{x} d x \\
2.3 \int x e^{x} d x & = & 2.3 x e^{x}-1.2 .3 \int e^{x} d x \\
-1.2 .3 & \int^{x} d x & =-1.2 .3 . e^{x} \\
\int x^{8} e^{x} d x & =e^{x}\left(x^{3}-3 x^{2}+2.3 x-1.2 .3\right) .
\end{array}
$$

32) $\int x^{4}(1+x)^{3} d x=\frac{x^{5}}{5}(1+x)^{3}-\frac{3}{5} \int x^{5}(1+x)^{2} d x$;

$\int x^{5}(1+x)^{2} d x=\frac{x^{6}}{6}(1+x)^{2}-\frac{2}{6} \int x^{6}(1+x) d x ;$

$\int x^{6}(1+x) d x=\frac{x^{7}}{7}+\frac{x^{8}}{8}$

$\int x^{4}(1+x)^{3} d x=\frac{x^{5}}{5}(1+x)^{3}-\frac{3 x^{6}}{5.6}(1+x)^{2}+\frac{3.2}{5.6}\left(\frac{x^{7}}{7}+\frac{x^{8}}{8}\right)$.

33) $\int x \cdot \cos x d x=x \sin x-\int \sin x d x=x \sin x+\cos x$. 
34) $\int \cos ^{2} x d x=\int \cos x \cdot \cos x d x=\cos x \sin x+\int \sin ^{2} x d x$ $=\sin x \cos x+\int\left(1-\cos ^{2} x\right) d x ;$

$2 \int \cos ^{2} x d x=\sin x \cos x+\int 1 . d x ;$

$\int \cos ^{2} x d x=\frac{1}{2} \sin x \cos x+\frac{x}{2}$

35) $\int \sin ^{2} x d x=\frac{x}{2}-\frac{1}{2} \sin x \cos x$

36) $y=\int \frac{x^{2}}{\sqrt{1-x^{2}}} d x=-\int x \cdot \frac{-x}{\sqrt{1-x^{2}}} d x$

$\int \sqrt{1-x^{2}} d x=\int \frac{1-x^{2}}{\sqrt{1-x^{2}}} d x=\int \frac{1}{\sqrt{1-x^{2}}} d x-y$;

$2 y=-x \sqrt{1-x^{2}}+\arcsin x$,

$\int \frac{x^{2}}{\sqrt{1-x^{2}}} d x=-\frac{x}{2} \sqrt{1-x^{2}}+\frac{1}{2} \arcsin x$

37) $\int \frac{1}{x} \lg x d x=(\lg x)^{2}-\int \frac{1}{x} \lg x d x ; \int \frac{1}{x} \lg x d x=\frac{1}{2}(\lg x)^{2}$

38) $\int x^{3} \lg x d x=\frac{x^{4}}{4} \lg x-\frac{1}{4} \int x^{3} d x=\frac{x^{4}}{4}\left(\lg x-\frac{1}{4}\right)$

39) $\int x^{2} \cos x d x=x^{2} \sin x-2 \int x \sin x d x$;

$\int x \sin x d x=-x \cos x+\int \cos x d x$

$\int x^{2} \cos x d x=\left(x^{2}-2\right) \sin x+2 x \cos x$

40) $\int e^{x} \sin x d x=e^{x} \sin x-\int e^{x} \cos x d x$;

$\int e^{x} \cos x d x=e^{x} \cos x+\int e^{x} \sin x d x$.

Durch Subtraktion und Addition entsteht:

$\int e^{x} \sin x d x=\frac{e^{x}}{2}(\sin x-\cos x)$ bezw.

41) $\int e^{x} \cos x d x=\frac{e^{x}}{2}(\sin x+\cos x)$

42) $\int 1 \cdot \arcsin x d x=x \arcsin x-\int \frac{x}{\sqrt{1-x^{2}}} d x$

$=x \arcsin x+\sqrt{1-x^{2}}$. 


$$
\int \frac{f^{\prime}(x)}{f(x)} d x=\lg f(x)+C
$$

ist nur die Umkehrung von Aufgabe 195 Diff. $R$. und heisst in Worten: Ist der Zähler eines Bruches der Differentialquotient des Nenners, so ist das Integral des Bruches der natürliche Logarithmus des Nenners.
43) $\int \frac{a}{b+c x} d x=\frac{a}{c} \int \frac{c}{b+c x} d x=\frac{a}{c} \lg (b+c x)$
44) $\int \frac{x^{n-1}}{a+b x^{n}} d x=\frac{1}{n b} \lg \left(a+b x^{n}\right)$
45) $\int \frac{e^{x}}{e^{x}+a} d x=\lg \left(e^{x}+a\right)$
46) $\int \frac{\cos x}{\sin x} d x=\lg \sin x$
47) $\int \frac{\sin x}{a+b \cos x} d x=-\frac{1}{b} \lg (a+b \cos x)$.

§ 2. Integration rationaler algebraischer Bruche.

$$
\int \frac{\varphi(x)}{a x+b} d x \text {. }
$$

Nachdem durch Division mit dem Nenner in den Zähler der Bruch in einen ganzen Quotienten und einen Restbruch, dessen Zählen die Variabele nicht mehr enthält, zerlegt ist, werden die einzelnen Teile integriert.

Aufgaben.

48) $\begin{aligned} \int \frac{10 x^{4}-17 x^{3}-24 x^{2}+16 x-14}{2 x-5} d x=\int\left(5 x^{8}+4 x^{2}-2 x+3+\frac{1}{2 x-5}\right) d x \\ =\frac{5 x^{4}}{4}+\frac{4 x^{8}}{3}-x^{2}+3 x+\frac{1}{2} \lg (2 x-5)\end{aligned}$

49) $\int \frac{x^{4}-6 x^{8}+13 x^{2}-10 x+3}{x-2} d x=\frac{x^{4}}{4}-\frac{4 x^{3}}{3}+\frac{5 x^{2}}{2}+3 \lg (x-2)$

50) $\int \frac{2 x^{3}+7 x^{2}+4 x+2}{2 x+3} d x=\frac{x^{8}}{3}+x^{2}-x+\frac{5}{2} \lg (2 x+3)$

51) $\int \frac{x+a}{x+b} d x=x+(a-b) \lg (x+b)$ 
52) $\int \frac{12-28 x+17 x^{2}-10 x^{8}}{1-5 x} d x=\frac{2 x^{3}}{3}-\frac{3 x^{2}}{2}+5 x-\frac{7}{5} \lg (1-5 x)$.

Steht die Variabele unter einem Wurzelzeichen, so ist der Ausdruck zuerst durch Substitution rational zu machen.

53)

$$
\begin{aligned}
\int \frac{\sqrt{x}}{1+\sqrt{x}} d x & =2 \int \frac{z^{2}}{z+1} d z, \text { wenn } z=\sqrt{x} \\
& =2\left(\frac{x}{2}-\sqrt{x}+\lg (1+\sqrt{x})\right)
\end{aligned}
$$

$$
\text { 54) } \int \frac{5}{2+\sqrt{x}} d x=10 \sqrt{x}-20 \lg (2+\sqrt{x})
$$

$$
\begin{aligned}
\int \frac{x^{2} \sqrt{x}}{1+\sqrt{x}} d x= & \frac{x^{3}}{3}-\frac{2}{5} \sqrt{x^{5}}+\frac{x^{2}}{2}-\frac{2}{3} \sqrt{x^{3}} \\
& +x-2 \sqrt{x}+2 \lg (1+\sqrt{x}) . \\
& \int \frac{\varphi(x)}{x^{2}+2 a x+b} d x .
\end{aligned}
$$

Wenn der Zähler von höherem Grade ist als der Nenner, so scheiden wir durch Division einen Quotienten ab und integrieren denselben Glied für Glied. Das Integral des noch ubrigen Restbruches hat im allgemeinen folgende Form:

$$
\int \frac{m x+n}{x^{2}+2 a x+b} d x \text {. }
$$

Zunächst lösen wir die Gleichung: $x^{2}+2 a x+b=0$, nennen die Wurzeln $\alpha$ und $\beta$, setzen $x^{2}+2 a x+b=(x-\alpha)(x-\beta)$ und unterscheiden 3 Fälle.

1) Die Wurzeln $\alpha$ und $\beta$ sind reell und versehieden. Der Bruch wird dann in Partialbrïche zerlegt wie folgt:

$$
\frac{m x+n}{x^{2}+2 a x+b}=\frac{m x+n}{(x-\alpha) \cdot(x-\beta)}=\frac{A}{x-\alpha}+\frac{B}{x-\beta} .
$$

Die Zähler $A$ und $B$ sind nun so zu bestimmen, dass die Gleichung identisch richtig ist, $\mathrm{d}$. h. dass die beiden Seiten für jeden beliebigen Wert von $x$ gleich sind. Wir setzen:

$$
\frac{m x+n}{x-\beta}=A+\frac{B(x-\alpha)}{x-\beta}
$$


wählen $x=\alpha$ und finden $\frac{m \alpha+n}{\alpha-\beta}=A$. Auf entsprechende Weise findet man auch $B$; doch kann $B$ auch aus $A$ dadurch abgeleitet werden, dass man $\alpha$ mit $\beta$ vertauscht. $B=-\frac{m \beta+n}{\alpha-\beta}$.

$$
\frac{m x+n}{(x-\alpha)(x-\beta)}=\frac{\frac{m \alpha+n}{\alpha-\beta}}{x-\alpha}-\frac{\frac{m \beta+n}{\alpha-\beta}}{x-\beta}
$$

$\int \frac{m x+n}{x^{2}+2 a x+b} d x=\frac{(m \alpha+n) \lg (x-\alpha)-(m \beta+n) \lg (x-\beta)}{\alpha-\beta}$.

Aufgaben.

56) $\int \frac{x+13}{x^{2}-4 x-5} d x=3 \lg (x-5)-2 \lg (x+1)$.

57) $\int \frac{2 x+6}{2 x^{2}+3 x+1} d x=5 \lg \left(x+\frac{1}{2}\right)-4 \lg (x+1)$

58) $\int \frac{6 x-13}{x^{2}-\frac{7}{2} x+\frac{3}{2}} d x=2 \lg (x-3)+4 \lg \left(x-\frac{1}{2}\right)$

59) $\int \frac{\frac{5}{6} x-16}{x^{2}+3 x-18} d x=\frac{7}{3} \lg (x+6)-\frac{3}{2} \lg (x-3)$

60) $\int \frac{3 x^{3}+5 x^{2}-29 x-25}{x^{2}+x-12} d x=\frac{3 x^{2}}{2}+2 x+2 \lg (x-3)+3 \lg (x+4)$

61) $\int \frac{\sqrt[3]{x^{2}}-\sqrt{x}+1}{\sqrt[3]{x}-1} d x=\frac{3 x^{\frac{4}{3}}}{4}-\frac{6 x^{\frac{7}{6}}}{7}+x-\frac{6 x^{\frac{5}{6}}}{5}+3 x^{\frac{2}{3}}$ $-2 x^{\frac{1}{2}}+6 x^{\frac{1}{3}}-6 x^{\frac{1}{b}}+3 \lg \left(x^{\frac{1}{t}}-1\right)+9 \lg \left(x^{\frac{1}{t}}+1\right)$. Man setze $x^{\frac{1}{6}}=z$.

62) $\int \frac{1}{x^{2}-a^{2}} d x=\frac{1}{2 a} \lg \frac{x-a}{x+a}$

63) $\int \frac{1}{x^{2}+2 x-1} d x=\int \frac{1}{(x+1)^{2}-2} d x=\frac{1}{2 \sqrt{2}} \lg \frac{x+1-\sqrt{2}}{x+1+\sqrt{2}}$

64) $\int \frac{1}{x^{2}+a^{2}} d x=\int \frac{1}{x^{2}-(a i)^{2}} d x=\frac{1}{2 a i} \lg \frac{x-a i}{x+a i}$.

Es ist aber auch bereits gefunden:

$$
\int \frac{1}{x^{2}+a^{2}} d x=\frac{1}{a} \operatorname{arctg} \frac{x}{a},
$$


und da wir wissen, dass zwei Integralwerte der nämlichen Funktion nur um eine Konstante differieren können, so ist es gestattet zu setzen: $\frac{1}{2 i} \lg \frac{x-a i}{x+a i}=\operatorname{arctg} \frac{x}{a}+C$. Wird diese Formel zur Verwandlung von Integralwerten benutzt, so kann die Konstante $C$ in die willkürliche Konstante des Integrals aufgenommen werden. Setzen wir nun noch $x-p$ für $x$ und $q$ für $a$, so entsteht die Formel:

$$
\frac{1}{2 i} \lg \frac{x-p-q i}{x-p+q i}=\operatorname{arctg} \frac{x-p}{q}+C,
$$

die bald Verwendung finden wird (vgl. S. 99).

2) Die beiden Wurzeln $\alpha$ und $\beta$ sind reell und gleich. Dann nimmt die Formel (4) die Form $\frac{0}{0}$ an und erhält ihren wahren Wert, wenn man Zähler und Nenner nach $\beta$ differentiiert und dann $\beta=\alpha$ setzt. So wird gefunden:

$$
\begin{aligned}
\int \frac{m x+n}{(x-\alpha)^{2}} d x & =-\left[\frac{m \lg (x-\beta)-\frac{m \beta+n}{x-\beta}}{-1}\right]_{\beta=\alpha} \\
= & m \lg (x-\alpha)-\frac{m \alpha+n}{x-\alpha} .
\end{aligned}
$$

Will man diese Formel direkt ableiten, so setzt man:

$$
\frac{m x+n}{(x-\alpha)^{2}}=\frac{A}{(x-\alpha)^{2}}+\frac{B}{x-\alpha},
$$

oder $m x+n=A+B(x-\alpha)$ und findet $B=m$ und $A=m \alpha+n$. Die beiden Teile sind dann leicht zu integrieren. Man könnte auch so verfahren:

$$
\begin{aligned}
\int \frac{m x+n}{(x-\alpha)^{2}} d x=\frac{m}{2} \int \frac{2(x-\alpha)}{(x-\alpha)^{2}} d x+ & \int \frac{m \alpha+n}{(x-\alpha)^{2}} d x \\
& =m \lg (x-\alpha)-\frac{m \alpha+n}{x-\alpha} .
\end{aligned}
$$
65) $\int \frac{2 x-13}{(x-5)^{2}} d x=2 \lg (x-5)+\frac{3}{x-5}$
66) $\int \frac{3 x+1}{(x+2)^{2}} d x=3 \lg (x+2)+\frac{5}{x+2}$. 
3) Die beiden Wurzeln $\alpha$ und $\beta$ der Gleichung $x^{2}+2 a x+b=0$ sind complex und von der Form:

$$
\alpha=p+q i, \beta=p-q i \text {. }
$$

Es ist dann $p=-a, q=\sqrt{b-a^{2}}$, und Formel (4) gestaltet sich unter Verwendung von (5) wie folgt:

$\int \frac{m x+n}{x^{2}+2 a x+b} d x=\int \frac{m x+n}{(x-p-q i)(x-p+q i)} d x$

$=\frac{\{m(p+q i)+n\} \lg (x-p-q i)-\{m(p-q i)+n\} \lg (x-p+q i)}{2 q i}$

$=\frac{m p+n}{q} \cdot \frac{1}{2 i} \lg \frac{x-p-q i}{x-p+q i}+\frac{m}{2} \lg [(x-p-q i)(x-p+q i)]$

$=\frac{m}{2} \lg \left(x^{2}+2 a x+b\right)+\frac{m p+n}{q} \operatorname{arctg} \frac{x-p}{q}+C$.

Übrigens kann diese Formel auch direkt entwickelt werden:

$\int \frac{m x+n}{x^{2}+2 a x+b} d x=\frac{m}{2} \int \frac{2 x+2 a}{x^{2}+2 a x+b} d x+\int \frac{n-a m}{x^{2}+2 a x+b} d x$

$$
=\frac{m}{2} \lg \left(x^{2}+2 a x+b\right)+\int \frac{n-a m}{(x+a)^{2}+\left(b-a^{2}\right)} d x .
$$

Da nach der Voraussetzung $\sqrt{b-a^{2}}$ reell ist, so ist die Substitution $(a+x)=z \sqrt{b-a^{2}}$ möglich, und wir erhalten:

$\int \frac{n-a m}{(x+a)^{2}+\left(b-a^{2}\right)} d x=\frac{n-a m}{\sqrt{b-a^{2}}} \int \frac{1}{z^{2}+1} d z=\frac{n-a m}{\sqrt{b-a^{2}}} \operatorname{arctg} z$. So findet man sohliesslich:

$\int \frac{m x+n}{x^{2}+2 a x+b} d x=\frac{m}{2} \lg \left(x^{2}+2 a x+b\right)+\frac{n-a m}{\sqrt{b-a^{2}}} \operatorname{arctg} \frac{x+a}{\sqrt{b-a^{2}}}$. Diese Formel stimmt bis auf die Integrations-Konstante mit (7) uberein, weil dort $p=-a, q=\sqrt{b-a^{2}}$ gesetzt ist.

\section{Aufgaben.}

67) $\int \frac{2 u-10}{u^{2}+2 u+10} d u=\lg \left(u^{2}+2 u+10\right)-4 \operatorname{arctg} \frac{u+1}{3}$

68) $\int \frac{2 u-20}{u^{2}-8 u+25} d u=\lg \left(u^{2}-8 u+25\right)-4 \operatorname{arctg} \frac{u-4}{3}$ 
69) $\int \frac{3 u+4}{u^{2}+4 u+8} d u=\frac{3}{2} \lg \left(u^{2}+4 u+8\right)-\operatorname{arctg} \frac{u+2}{2}$

70) $\int \frac{u+6}{u^{2}-3} d u=\frac{1}{2} \lg \left(u^{2}-3\right)+\sqrt{3} \lg \frac{u-\sqrt{3}}{u+\sqrt{3}}$

71) $\int \frac{u+6}{u^{2}+3} d u=\frac{1}{2} \lg \left(u^{2}+3\right)+2 \sqrt{3} \operatorname{arctg} \frac{u}{\sqrt{3}}$

72) $\int \frac{6 u}{u^{2}+4 u+13} d u=3 \lg \left(u^{2}+4 u+13\right)-4 \operatorname{arctg} \frac{u+2}{3}$

73) $\int \frac{10 u-44}{u^{2}-4 u+20} d u=5 \lg \left(u^{2}-4 u+20\right)-6 \operatorname{arctg} \frac{u-2}{4}$

74) $\int \frac{u^{2}}{5 u^{2}+12} d u=\frac{1}{5} u-\frac{1}{25} \sqrt{60} \operatorname{arctg} u \sqrt{\frac{5}{12}}$

75) $\int \frac{4 u-5}{u^{2}-6 u+10} d u=2 \lg \left(u^{2}-6 u+10\right)+7 \operatorname{arctg}(u-3)$

76) $\int \frac{27 u^{6}}{3 u^{2}+2} d u=\frac{9 u^{5}}{5}-2 u^{3}+4 u-\frac{8}{\sqrt{6}} \operatorname{arctg} u \sqrt{\frac{3}{2}}$

77) $\int \frac{1}{a+b u^{2}} d u=\frac{1}{\sqrt{a b}} \operatorname{arctg} u \sqrt{\frac{b}{a}}$, wenn $a b>0$ ist.

Integration eines Bruches von der Form:

$$
\frac{\varphi(x)}{f(x)}=\frac{a_{0} x^{m}+a_{1} x^{m-1}+a_{2} x^{m-2}+\ldots+a_{m}}{x^{n}+b_{1} x^{n-1}+b_{2} x^{n-2}+\ldots+b_{n}} .
$$

Es wird vorausgesetzt, dass $m<n$ ist. Sollte diese Bedingung bei einem gegebenen Bruche noch nicht erfullt sein, so kann man die Division, so lange es geht, ausführen. Der gegebene Bruch wird dann gleich einer ganzen Funktion nebst einem angehängten Bruch von der Form $\frac{\varphi(x)}{f(x)}$, in welchem $m<n$ ist. Vor allem anderen muss nun der Nenner in Binomialfaktoren zerlegt und darum die Gleichung aufgelöst werden:

$$
f(x)=x^{n}+b_{1} x^{n-1}+b_{2} x^{n-2}+\ldots+b_{n}=0
$$

Wir haben bei einer Wurzel $\alpha$ zwei Fälle zu unterscheiden.

1) Eine der Wurzeln $\alpha$ von $f(x)=0$ ist von den thbrigen verschieden, oder mit anderen Worten, $f(x)$ ist durch $(x-\alpha)$ teilbar aber nicht durch $(x-\alpha)^{2}$, so dass wir 
setzen können $f(x)=(x-\alpha) g(x)$, wo dann $g(x)=0$ die Wurzel $\alpha$ nicht mehr besitzt. Wir setzen

Daraus folgt

$$
\frac{\varphi(x)}{f(x)}=\frac{\varphi(x)}{(x-\alpha) g(x)}=\frac{A}{x-\alpha}+\frac{\psi(x)}{g(x)} .
$$

$$
\varphi(x)=A g(x)+\psi(x)(x-\alpha)
$$

und für $x=\alpha$ findet man $A=\varphi(\alpha): g(\alpha)$. Nun ist aber

und

$$
f^{\prime}(x)=(x-\alpha) g^{\prime}(x)+g(x) \text { also } f^{\prime}(\alpha)=g(\alpha)
$$

$$
\begin{gathered}
A=\frac{\varphi(\alpha)}{f^{\prime}(\alpha)} \\
\frac{\varphi(x)}{f(x)}=\frac{\varphi(\alpha)}{f^{\prime}(\alpha)} \frac{1}{x-\alpha}+\frac{\varphi(x)}{g(x)} .
\end{gathered}
$$

Einen solehen Bruch wie den ersten rechts nennt man dann einen Partialbruch. Das Problem der Integration ist hierdurch auf ein einfacheres zurückgeführt, da $g(x)$ nur vom Grade $(n-1)$ ist. Wenn $f(x)=0$ uberhaupt nur von einander verschiedene Wurzeln $\alpha_{1}, \alpha_{2}, \ldots \alpha_{n}$ enthält, ist es tiberhaupt erledigt. In diesem allgemeinen Falle wird

$$
\int \frac{\varphi(x)}{f(x)} d x=\frac{\varphi(\alpha)}{f^{\prime}(\alpha)} \lg (x-\alpha)+\int \frac{\psi(x)}{g(x)} d x,
$$

und für $f(x)=\left(x-\alpha_{1}\right)\left(x-\alpha_{2}\right) \ldots\left(x-\alpha_{n}\right)$ erhält man, da jetzt der gegebene Bruch vollständig in Partialbrüche zerlegt werden kann,

$$
\begin{aligned}
\int \frac{\varphi(x)}{f(x)} d x=\frac{\varphi\left(\alpha_{1}\right)}{f^{\prime}\left(\alpha_{1}\right)} \lg \left(x-\alpha_{1}\right)+\frac{\varphi\left(\alpha_{2}\right)}{f^{\prime}\left(\alpha_{2}\right)} \lg \left(x-\alpha_{2}\right)+\ldots & \\
& +\frac{\varphi\left(\alpha_{n}\right)}{f^{\prime}\left(\alpha_{n}\right)} \lg \left(x-\alpha_{n}\right)
\end{aligned}
$$

Beispiel: $\frac{\varphi(x)}{f(x)}=\frac{7 x^{2}+7 x-176}{x^{8}-9 x^{2}+6 x+56}$;

$$
\begin{gathered}
a_{1}=-2, \alpha_{2}=4, \alpha_{3}=7 ; f^{\prime}(x)=3 x^{2}-18 x+6 \\
\frac{\varphi(x)}{f(x)}=\frac{A_{1}}{x+2}+\frac{A_{2}}{x-4}+\frac{A_{8}}{x-7} \\
A_{1}=\frac{\varphi(-2)}{f^{\prime}(-2)}=-3, A_{2}=\frac{\varphi(4)}{f^{\prime}(4)}=2, A_{8}=\frac{\varphi(7)}{f^{\prime}(7)}=8 \\
\frac{\varphi(x)}{f(x)}=-\frac{3}{x+2}+\frac{2}{x-4}+\frac{8}{x-7} .
\end{gathered}
$$


78) $\int \frac{7 x^{2}+7 x-176}{x^{3}-9 x^{2}+6 x+56} d x=-3 \lg (x+2)+2 \lg (x-4)+8 \lg (x-7)$. A ufg a ben.

79) $\int \frac{5 u^{2}-7 u+13}{u^{3}-6 u^{2}+11 u-6} d u=\frac{11}{2} \lg (u-1)-19 \lg (u-2)+\frac{37}{2} \lg (u-3)$

80) $\int \frac{u^{2}+5 u+41}{(u+3)(u-1)\left(u-\frac{1}{2}\right)} d u=\frac{5}{2} \lg (u+3)+\frac{47}{2} \lg (u-1)-25 \lg \left(u-\frac{1}{2}\right)$

81) $\int \frac{1}{u^{3}-a^{2} u} d u=\frac{1}{2 a^{2}} \lg \left(u^{2}-a^{2}\right)-\frac{1}{a^{2}} \lg u$

82) $\int \frac{10 u^{3}+40 u^{2}+40 u+6}{u^{4}+6 u^{3}+11 u^{2}+6 u} d u=\lg u+2 \lg (u+1)$ $+3 \lg (u+2)+4 \lg (u+3)$

83) $\int \frac{17 u^{2}-u-26}{\left(u^{2}-1\right)\left(u^{2}-4\right)} d u=\frac{5}{3} \lg (u-1)-\frac{4}{3} \lg (u+1)$

$$
+\frac{10}{3} \lg (u-2)-\frac{11}{3} \lg (u+2)
$$

84) $\int \frac{4 u^{3}+9 u^{2}-270 u+653}{(u-3)(u-4)(u-7)(u+5)} d u=\lg \left(u^{2}-7 u+12\right)$ $+4 \lg (u-7)-2 \lg (u+5)$

85) $\int \frac{u^{2}-3 u+3}{u^{8}-4 u^{2}-7 u+10} d u=\frac{13}{21} \lg (u+2)+\frac{13}{28} \lg (u-5)-\frac{1}{12} \lg (u-1)$ 86) $\int \frac{5 u^{2}-7 a u+11 a^{2}}{u^{3}-6 a u^{2}+11 a^{2} u-6 a^{3}} d u=\frac{9}{2} \lg (u-a)-17 \lg (u-2 a)$ $+\frac{35}{2} \lg (u-3 a)$ 87) $\int \frac{2 u^{3}+12 a u^{2}-8 a^{2} u-12 a^{3}}{\left(u^{2}-a^{2}\right)\left(u^{2}-4 a^{2}\right)} d u=\lg \left(u^{2}-a^{2}\right)+3 \lg \frac{u-2 a}{u+2 a}$ 88) $\int \frac{2 u^{4}-10 u^{3}+21 u^{2}-20 u+5}{u^{2}-3 u+2} d u=\frac{2 u^{3}}{3}-2 u^{2}+5 u$ $+\lg (u-2)+2 \lg (u-1)$.

Wenn die Koefficienten in $f(x)$ reell sind, aber unter den Wurzeln $\alpha_{1}, \alpha_{2} \ldots \alpha_{n}$ sich auch complexe Werte befinden, so müssen diese paarweise konjugiert vorkommen und die Form haben: $\alpha=p+q i, \beta=p-q i$. Die Zerlegung in Partialbrïche lässt sich mit folgenden Modifikationen auch hier nach der besprochenen Methode durchführen. 
Man hat

$$
\frac{\varphi(x)}{f(x)}=\frac{\varphi(p+q i)}{f^{\prime}(p+q i)} \frac{1}{x-p-q i}+\frac{\varphi(p-q i)}{f^{\prime}(p-q i)} \frac{1}{x-p+q i}+\ldots
$$
und wenn wir $\frac{\varphi(p+q i)}{f^{\prime}(p+q)}=s+t i$ und also $\frac{\varphi(p-q}{f^{\prime}(p-q)}=s-t i$
setzen,

$$
\begin{aligned}
\int \frac{\varphi(x)}{f(x)} d x & =(s+t i) \lg (x-p-q i)+(s-t i) \lg (x-p+q i)+\ldots \\
& =s \lg \left[(x-p)^{2}+q^{2}\right]+t i \lg \frac{x-p-q i}{x-p+q i}+\ldots,
\end{aligned}
$$

wo rechts eine beliebige Integrationskonstante addiert werden kann. Benutzt man nun die auf S. 119 bei Formel (5) gemachte Bemerkung, dann folgt

$t i \lg \frac{x-p-q i}{x-p+q i}=-2 t \cdot \frac{1}{2 i} \lg \frac{x-p-q i}{x-p+q i}$

$$
=-2 t \operatorname{arctg} \frac{x-p}{q}+C .
$$

So gelangen wir von dem oben gegebenen komplexen zu dem reellen Ausdrucke

$$
\int \frac{\varphi(x)}{f(x)} d x=s \lg \left[(x-p)^{2}+q^{2}\right]-2 t \operatorname{arctg} \frac{x-p}{q}+\ldots
$$

Wir wollen dies an den folgenden Beispielen durchfuhren.

$$
\begin{gathered}
\frac{\varphi(x)}{f(x)}=\frac{2 x}{\left(x^{2}+1\right)\left(x^{2}+3\right)}=\frac{2 x}{(x-i)(x+i)(x-r i)(x+r i)}, r=\sqrt{3} \\
\frac{\varphi(x)}{f(x)}=\frac{A_{1}}{x-i}+\frac{A_{2}}{x+i}+\frac{A_{3}}{x-r i}+\frac{A_{4}}{x+r i} \\
A_{1}=\frac{\varphi(i)}{f^{\prime}(i)}=\frac{1}{2}, \quad A_{2}=\frac{\varphi(-i)}{f^{\prime}(-i)}=\frac{1}{2}, \quad A_{3}=\frac{\varphi(r i)}{f^{\prime}(r i)}=-\frac{1}{2}, \\
A_{4}=\frac{\varphi(-r i)}{f^{\prime}(-r i)}=-\frac{1}{2} \\
\frac{\varphi(x)}{f(x)}=\frac{\frac{1}{2}}{x-i}+\frac{\frac{1}{2}}{x+i}-\frac{\frac{1}{2}}{x-r i}-\frac{\frac{1}{2}}{x+r i} \\
\int \frac{\varphi(x)}{f(x)} d x=\frac{1}{2}[\lg (x-i)+\lg (x+i)]-\frac{1}{2}[\lg (x-r i)+\lg (x+r i)] \\
\quad=\frac{1}{2} \lg \left(x^{2}+1\right)-\frac{1}{2} \lg \left(x^{2}-r^{2} i^{2}\right)
\end{gathered}
$$


89) $\int \frac{2 x}{\left(x^{2}+1\right)\left(x^{2}+3\right)} d x=\frac{1}{2} \lg \frac{x^{2}+1}{x^{2}+3}$.

$$
\begin{gathered}
\frac{\varphi(x)}{f(x)}=\frac{10 x^{3}+110 x+400}{\left(x^{2}-4 x+29\right)\left(x^{2}-2 x+5\right)} \\
=\frac{A_{1}}{x-2-5 i}+\frac{A_{2}}{x-2+5 i}+\frac{A_{3}}{x-1-2 i}+\frac{A_{4}}{x-1+2 i} \\
A_{1}=\frac{\varphi(2+5 i)}{f^{\prime}(2+5 i)}=2-3 i, \quad A_{2}=\frac{\varphi(2-5 i)}{f^{\prime}(2-5 i)}=2+3 i, \\
A_{3}=\frac{\varphi(1+2 i)}{f^{\prime}(1+2 i)}=3-4 i, \quad A_{4}=\frac{\varphi(1-2 i)}{f^{\prime}(1-2 i)}=3+4 i ;
\end{gathered}
$$

$\frac{\varphi(x)}{f(x)}=\frac{2-3 i}{x-2-5 i}+\frac{2+3 i}{x-2+5 i}+\frac{3-4 i}{x-1-2 i}+\frac{3+4 i}{x-1+2 i}$ $\int \frac{\varphi(x)}{f(x)} d x=(2-3 i) \lg (x-2-5 i)+(2+3 i) \lg (x-2+5 i)$ $+(3-4 i) \lg (x-1-2 i)+(3+4 i) \lg (x-1+2 i)$ $=2 \lg [(x-2-5 i)(x-2+5 i)]+3 \lg [(x-1-2 i)(x-1+2 i)]$ $-3 i \lg \frac{x-2-5 i}{x-2+5 i}-4 i \lg \frac{x-1-2 i}{x-1+2 i}$.

Multipliziert man die Faktoren aus und wendet auf die beiden letzten Teile Formel (5), S. 119, an, so giebt es:

90) $\int \frac{10 x^{3}+110 x+400}{\left(x^{2}-4 x+29\right)\left(x^{2}-2 x+5\right)} d x=2 \lg \left(x^{2}-4 x+29\right)$ $+3 \lg \left(x^{2}-2 x+5\right)+6 \operatorname{arctg} \frac{x-2}{5}+8 \operatorname{arctg} \frac{x-1}{2}$.

Die besprochene Methode kommt im Grunde darauf hinaus, einen Bruch von der Form $\frac{m x+n}{x^{2}+2 a x+b}$ zu integrieren, wenn $b>a^{2}$ ist. Das kann auch direkt so geschehen. $\int \frac{m x+n}{x^{2}+2 a x+b} d x=\frac{1}{2} m \int \frac{(2 x+2 a) d x}{x^{2}+2 a x+b}+(n-m a) \int \frac{d x}{(x+a)^{2}+\left(b-a^{2}\right)}$ $=\frac{1}{2} m \lg \left(x^{2}+2 a x+b\right)+\frac{n-m a}{\sqrt{b-a^{2}}} \int \frac{d z}{1+z^{2}}$, wenn $\sqrt{b-a^{2}} z=x+a$ ist. Also erhält man $\int \frac{m x+n}{x^{2}+2 a x+b} d x=\frac{1}{2} m \lg \left(x^{2}+2 a x+b\right)+\frac{n-m a}{\sqrt{b-a^{2}}} \operatorname{arctg} \frac{x+a}{\sqrt{b-a^{2}}}$. 


$$
\frac{\varphi(x)}{f(x)}=\frac{8 x^{2}-29 x+61}{(x-1)\left(x^{2}-6 x+13\right)}=\frac{A}{x-1}+\frac{m x+n}{x^{2}-6 x+13}
$$
$A=\frac{\varphi(1)}{f^{\prime}(1)}=5$. Dieser Wert wird sofort benutzt :

$\frac{8 x^{2}-29 x+61}{(x-1)\left(x^{2}-6 x+13\right)}=\frac{5\left(x^{2}-6 x+13\right)+(m x+n)(x-1)}{(x-1)\left(x^{2}-6 x+13\right)}$

Nach dem Satze von den unbestimmten Koefficienten ergeben sich aus den Zählern die Relationen: $8=5+m, 61=65-n$, oder $m=3, n=4$.

$$
\frac{\varphi(x)}{f(x)}=\frac{5}{x-1}+\frac{3 x+4}{x^{2}-6 x+13} .
$$

91) $\int \frac{8 x^{2}-29 x+61}{(x-1)\left(x^{2}-6 x+13\right)} d x=5 \lg (x-1)+\frac{3}{2} \lg \left(x^{2}-6 x+13\right)$ $+\frac{13}{2} \operatorname{arctg} \frac{x-3}{2}$.

$$
\frac{\varphi(x)}{f(x)}=\frac{1}{x^{4}+x^{2}+1}=\frac{m x+n}{x^{2}+x+1}+\frac{r x+t}{x^{2}-x+1} .
$$

Nachdem die rechte Seite gleichnamig gemacht ist, werden die Zähler gleichgesetzt.

$$
1=(m x+n)\left(x^{2}-x+1\right)+(r x+t)\left(x^{2}+x+1\right) ;
$$

$m+r=0, n-m+r+t=0, m-n+r+t=0, n+t=1$;

$$
m=\frac{1}{2}, n=\frac{1}{2}, t=\frac{1}{2}, r=-\frac{1}{2}
$$

$$
\frac{1}{x^{4}+x^{2}+1}=\frac{\frac{1}{2} x+\frac{1}{2}}{x^{2}+x+1}-\frac{\frac{1}{2} x-\frac{1}{2}}{x^{2}-x+1}
$$

92) $\int \frac{1}{x^{4}+x^{2}+1} d x=\frac{1}{4} \lg \frac{x^{2}+x+1}{x^{2}-x+1}+\frac{1}{2 \sqrt{3}} \operatorname{arctg} \frac{2 x+1}{\sqrt{3}}$ $+\frac{1}{2 \sqrt{3}} \operatorname{arctg} \frac{2 x-1}{\sqrt{3}}$

93) $\int \frac{15 x^{2}+66 x+21}{(x-1)\left(x^{2}+4 x+29\right)} d x=3 \lg (x-1)+6 \lg \left(x^{2}+4 x+29\right)$ $+\frac{42}{5} \operatorname{arctg} \frac{x+2}{5}$

94) $\int \frac{3 x^{2}-5 x+2}{x^{3}-2 x^{2}+3 x-6} d x=\frac{4}{7} \lg (x-2)+\frac{17}{14} \lg \left(x^{2}+3\right)$ $-\frac{1}{7 \sqrt{3}} \operatorname{arctg} \frac{x}{\sqrt{3}}$ 
95) $\int \frac{7 x^{2}+6 x-16}{(x-3)\left(x^{2}+4\right)} d x=5 \lg (x-3)+\lg \left(x^{2}+4\right)+6 \operatorname{arctg} \frac{x}{2}$

96) $\int \frac{2 x^{3}+28 x^{2}-257 x+531}{\left(x^{2}-16 x+69\right)\left(x^{2}-6 x+16\right)} d x=\frac{5}{2} \lg \left(x^{2}-16 x+69\right)$

$+\frac{43}{\sqrt{5}} \operatorname{arctg} \frac{x-8}{\sqrt{5}}-\frac{3}{2} \lg \left(x^{2}-6 x+16\right)-\frac{2}{\sqrt{7}} \operatorname{arctg} \frac{x-3}{\sqrt{7}}$

2. Eine der Wurzeln $\alpha$ von $f(x)=0$ kommt in der Multiplicität $r$ vor, oder mit anderen Worten $f(x)$ ist durch $(x-\alpha)^{r}$ aber nicht mehr durch $(x-\alpha)^{r+1}$ teilbar, so dass wir setzen können $f(x)=g(x)(x-\alpha)^{r}$, wo dann $g(x)=0$ die Wurzel $\alpha$ nicht mehr besitzt. Wir setzen

$\frac{\varphi(x)}{f(x)}=\frac{\varphi(x)}{(x-\alpha)^{r} g(x)}=\frac{A_{1}}{(x-\alpha)^{r}}+\frac{A_{2}}{(x-\alpha)^{r-1}}+\cdots+\frac{A_{r}}{x-\alpha}+\frac{\psi(x)}{g(x)}$, und suchen die Zähler $A_{1}, A_{2}, \ldots A_{r}$ der ersten Partialbrïche $\mathrm{zu}$ bestimmen. Es ist nicht praktisch, allgemeine Formeln aufzustellen und nach diesen zu rechnen; vorzuziehen ist der folgende Weg. Wir setzen $x-\alpha=y$ und bilden

$$
\frac{\varphi(y+\alpha)}{g(y+\alpha)}=A_{1}+A_{2} y+. .+A_{r} y^{r-1}+\frac{\psi(y+\alpha)}{g(y+\alpha)} y^{r}
$$

dann dividieren wir auf gewöhnliche Art, nach aufsteigenden Potenzen von $y$ geordnet $\varphi(y+\alpha)$ durch $g(y+\alpha)$ bis zur $(r-1)^{\text {ten }}$ Potenz von $y$; die erhaltenen Partial-Quotienten geben nach der Methode der unbestimmten Koeffizienten die Grössen $A$.

1. Beispiel. $\frac{x+2}{x^{4}+x^{3}}=\frac{A_{1}}{x^{3}}+\frac{A_{2}}{x^{2}}+\frac{A_{3}}{x}+\frac{\psi(x)}{x+1}$. Hier ist die Einfuhrung von $y$ tuberflussig. Dividiert man

so findet sich

$$
\frac{2+x}{1+x}=2-x+x^{2}-x^{3}+\cdots
$$

$$
\frac{x+2}{x^{4}+x^{3}}=\frac{2}{x^{3}}-\frac{1}{x^{2}}+\frac{1}{x}-\frac{1}{x+1} .
$$

Fur $\psi(x)$ ergeben nämlich die früheren Methoden den Wert -1 . 
2. Beispiel. $\frac{x^{2}+x+1}{(x-2)^{2}(x+1)}=\frac{A_{1}}{(x-2)^{2}}+\frac{A_{2}}{x-2}+\frac{\phi(x)}{x+1}$ $\frac{y^{2}+5 y+7}{y+3}=A_{1}+A_{2} y+\frac{\phi(y+2)}{y+3} y^{2}$, für $x=y+2$.

Die Division

$$
\frac{7+5 y+y^{2}}{3+y}=\frac{7}{3}+\frac{8}{8} y+\frac{1}{27} y^{2}+\cdots
$$

liefert

$$
\frac{x^{2}+x+1}{(x-2)^{2}(x+1)}=\frac{7}{3(x-2)^{2}}+\frac{8}{9(x-2)}+\frac{1}{9(x+1)}
$$

die früheren Methoden ergeben nämlich $\phi(x)=\frac{1}{9}$.

Hat man diese Zerfällung in Partialbrüche gemacht, so kann jeder einzelne integriert werden.

$\frac{5 u^{3}-11 u^{2}+5 u+4}{(u-1)^{4}}=\frac{A_{1}}{(u-1)^{4}}+\frac{A_{2}}{(u-1)^{3}}+\frac{A_{8}}{(u-1)^{2}}+\frac{A_{4}}{u-1} ;$

unsere Methode giebt hier sehr bequem für $u-1=y$

$$
3-2 y+4 y^{2}+5 y^{8}=A_{1}+A_{2} y+A_{3} y^{2}+A_{4} y^{3},
$$

weil $g(x)$ nicht vorhanden ist. Aus dieser Gleichung können wir sofort die Zahlen-Werte der vier Koeffizienten $A$ entnehmen. Das ergiebt

$$
A_{1}=3, A_{2}=-2, A_{3}=4, A_{4}=5 ;
$$

$\frac{5 u^{3}-11 u^{2}+5 u+4}{(u-1)^{4}}=\frac{3}{(u-1)^{4}}-\frac{2}{(u-1)^{3}}+\frac{4}{(u-1)^{2}}+\frac{5}{u-1}$

97) $\int \frac{5 u^{3}-11 u^{2}+5 u+4}{(u-1)^{4}} d u=-\frac{1}{(u-1)^{3}}+\frac{1}{(u-1)^{2}}-\frac{4}{u-1}$

$+5 \lg (u-1)$

98) $\int \frac{u^{3}-4 u^{2}+1}{(u-2)^{2}} d u=\frac{u^{2}}{2}-4 \lg (u-2)+\frac{7}{u-2}$

99) $\int \frac{2 u^{5}-u^{4}+4 u^{2}-5 u+1}{(u-3)^{6}} d u=2 \lg (u-3)-\frac{29}{u-3}$

$$
-\frac{84}{(u-3)^{2}}-\frac{490}{3(u-3)^{3}}-\frac{721}{4(u-3)^{4}}-\frac{427}{5(u-3)^{6}}
$$


100) $\int \frac{3 u^{2}-17 u+21}{(u-2)^{3}} d u=\frac{1}{2(u-2)^{2}}+\frac{5}{u-2}+3 \lg (u-2)$

101) $\int \frac{6 u^{3}+89 u^{2}+438 u+719}{(u+5)^{4}} d u=-\frac{4}{3(u+5)^{3}}+\frac{1}{(u+5)^{2}}$

$+\frac{1}{u+5}+6 \lg (u+5)$.

Wenn der Zähler ein einfacher Wert ist, kann auch die Integration nach Teilen Anwendung finden.

102) $\int u^{n}(u-\alpha)^{-p} d u=-\frac{u^{n}(u-\alpha)^{-p+1}}{p-1}+\frac{n}{p-1} \int u^{n-1}(u-\alpha)^{-p+1} d u$ $\int u^{2}(u-\alpha)^{-4} d u=-\frac{u^{2}(u-\alpha)^{-3}}{3}+\frac{2}{3} \int u(u-\alpha)^{-3} d u$ $\int u(u-\alpha)^{-3} d u=-\frac{u(u-\alpha)^{-2}}{2}+\frac{1}{2} \int(u-\alpha)^{-2} d u$ $\int(u-\alpha)^{-2} d u=-(u-\alpha)^{-1}$

103) $\int \frac{u^{2}}{(u-\alpha)^{4}} d u=-\frac{1}{3}\left[\frac{u^{2}}{(u-\alpha)^{3}}+\frac{u}{(u-\alpha)^{2}}+\frac{1}{u-\alpha}\right]$ 104) $\int \frac{u^{3}+a^{2} u-a^{3}}{(u-a)^{3}} d u=(u-a)-\frac{a^{3}}{2(u-a)^{2}}-\frac{4 a^{2}}{u-a}+3 a \lg (u-a)$ 105) $\int \frac{u^{2}}{(a+b u)^{3}} d u=-\frac{u^{2}}{2 b(a+b u)^{2}}-\frac{u}{b^{2}(a+b u)}+\frac{1}{b^{3}} \lg (a+b u)$ 106) $\int \frac{u^{2}}{\left(u^{2}+1\right)\left(u^{2}+4\right)} d u=\frac{1}{3} \int \frac{3 u^{2}}{\left(u^{2}+1\right)\left(u^{2}+4\right)} d u$ $=\frac{1}{3} \int \frac{4\left(u^{2}+1\right)-\left(u^{2}+4\right)}{\left(u^{2}+1\right)\left(u^{2}+4\right)} d u=\frac{1}{3} \int \frac{4}{u^{2}+4} d u$ $-\frac{1}{3} \int \frac{1}{u^{2}+1} d u=\frac{2}{3} \operatorname{arctg} \frac{u}{2}-\frac{1}{3} \operatorname{arctg} u$ $\frac{9 x^{4}-3 x^{3}-23 x^{2}+30 x-1}{(x-1)^{4}(x+3)}=\frac{3}{(x-1)^{4}}+\frac{2}{(x-1)^{3}}+\frac{5}{(x-1)^{2}}+\frac{7}{x-1}+\frac{2}{x+3}$ 107) $\int \frac{9 x^{4}-3 x^{3}-23 x^{2}+30 x-1}{(x-1)^{4}(x+3)} d x=-\frac{1}{(x-1)^{3}}-\frac{1}{(x-1)^{2}}$ $-\frac{5}{x-1}+7 \lg (x-1)+2 \lg (x+3)$ 
108) $\int \frac{x^{4}-7 x^{8}+3 x^{2}-11 x+13}{x^{3}(x+1)\left(x^{2}+x+1\right)} d x=-\frac{13}{2 x^{2}}+\frac{37}{x}+51 \lg x$ $-35 \lg (x+1)-8 \lg \left(x^{2}+x+1\right)-\frac{10}{\sqrt{3}} \operatorname{arctg} \frac{2 x+1}{\sqrt{3}}$

109) $\int \frac{x^{3}-2 x^{2}+7 x+4}{(x-1)^{2}(x+1)^{2}} d x=-\frac{5}{2(x-1)}-\lg (x-1)$ $+\frac{3}{2(x+1)}+2 \lg (x+1)$

110) $\int \frac{x^{3}-22 x^{2}+57 x+32}{(x+1)^{2}(x-3)^{2}} d x=\frac{3}{x+1}+5 \lg (x+1)-\frac{2}{x-3}$ $-4 \lg (x-3)$

111) $\int \frac{7 x^{3}-32 x^{2}+50 x-28}{(x-1)^{3}(x-4)} d x=-\frac{1}{2(x-1)^{2}}+\frac{2}{x-1}$ $+3 \lg (x-1)+4 \lg (x-4)$

112) $\int \frac{x^{3}+1}{x^{4}-3 x^{3}+3 x^{2}-x} d x=-\frac{1}{(x-1)^{2}}-\frac{1}{x-1}+2 \lg (x-1)-\lg x$

113) $\int \frac{3 x^{2}+x-2}{(x-1)^{3}\left(x^{2}+1\right)} d x=-\frac{1}{2(x-1)^{2}}-\frac{5}{2(x-1)}$ $-\frac{3}{2} \lg (x-1)+\frac{3}{4} \lg \left(x^{2}+1\right)-\operatorname{arctg} x$

114) $\int \frac{1}{x^{3}(x-1)^{2}(x+1)} d x=-\frac{1}{2 x^{2}}-\frac{1}{x}+2 \lg x$

$$
-\frac{1}{4} \lg (x+1)-\frac{1}{2(x-1)}-\frac{7}{4} \lg (x-1) .
$$

Die bisher entwickelte Zerlegung in Partialbrtiche ist auch noch ausführbar, wenn die gleichen Wurzeln komplex sind. $\frac{2 x+1}{\left(x^{2}+1\right)^{2}}=\frac{2 x+1}{(x-i)^{2}(x+i)^{2}}=\frac{A_{2}}{(x-i)^{2}}+\frac{A_{1}}{x-i}+\frac{B_{2}}{(x+i)^{2}}+\frac{B_{1}}{x+i}$ $=\frac{-\frac{1}{4} i}{x-i}+\frac{\frac{1}{4} i}{x+i}-\frac{\frac{2 i+1}{4}}{(x-i)^{2}}+\frac{\frac{2 i-1}{4}}{(x+i)^{2}}$.

115) $\int \frac{2 x+1}{\left(x^{2}+1\right)^{2}} d x=-\frac{1}{4} i \lg \frac{x-i}{x+i}-\frac{\frac{2 i-1}{4}}{x+i}+\frac{\frac{2 i+1}{4}}{x-i}$ $=\frac{1}{2} \operatorname{arctg} x+\frac{x-2}{2\left(x^{2}+1\right)}$ 


$$
\begin{aligned}
& \frac{x^{2}}{\left(x^{2}+1\right)^{3}}=\frac{\frac{i}{8}}{(x+i)^{3}}-\frac{\frac{1}{16}}{(x+i)^{2}}+\frac{\frac{i}{16}}{x+i} \\
&-\frac{\frac{i}{8}}{(x-i)^{3}}-\frac{\frac{1}{16}}{(x-i)^{2}}-\frac{\frac{i}{16}}{x+i}
\end{aligned}
$$

116) $\int \frac{x^{2}}{\left(x^{2}+1\right)^{3}} d x=\frac{1}{8} \frac{x^{3}-x}{\left(x^{2}+1\right)^{2}}+\frac{1}{8} \operatorname{arctg} x$.

Reduktionsformeln sind Formeln, durch welche eine Aufgabe auf eine andere leichtere von ähnlicher Gestalt reduziert wird. Sie können nicht selten dazu dienen, durch wiederholte Anwendung derartige Integrale entweder ganz auszuführen, oder doch so weit zu vereinfachen, dass ihre weitere Ausfuhrung nach den bisherigen Methoden sehr erleichtert ist.

Die Richtigkeit der nachfolgenden Reduktionsformel erkennt man leicht, indem man beide Seiten differentiiert. Man sieht dabei zugleich ein, dass die Zahlen $m, n, p$ beliebig sein können; nur darf $(n+1)$ nicht Null werden.

$$
\int x^{n}\left(a+b x^{m}\right)^{p} d x=\frac{x^{n+1}}{n+1}\left(a+b x^{m}\right)^{p}
$$

$$
-\frac{m b p}{n+1} \int x^{m+n}\left(a+b x^{m}\right)^{p-1} d x
$$

118) $\int x^{4}\left(a+b x^{2}\right)^{3} d x=\frac{x^{5}}{5}\left(a+b x^{2}\right)^{3}-\frac{6 b}{5} \int x^{6}\left(a+b x^{2}\right)^{2} d x$.

In dieser Gestalt kann die Formel dazu dienen, ein positives $p$ nach und nach so weit zu reduzieren, dass der Exponent kleiner als 1 wird. Soll dagegen ein negatives $p$ dem absoluten Werte nach verkleinert werden, so muss man die Formel 117) umkehren. Wird dann noch $p$ statt $p-1$ und $n$ statt $n+m$ gesetzit, so heisst sie:

119) $\begin{aligned} \int x^{n}\left(a+b x^{m}\right)^{p} d x & =\frac{x^{n-m+1}}{m b(p+1)}\left(a+b x^{m}\right)^{p+1} \\ & -\frac{n-m+1}{m b(p+1)} \int x^{n-m}\left(a+b x^{m}\right)^{p+1} d x .\end{aligned}$

Die Formel wird unbrauchbar für $p=-1$. 
120) $\int \frac{x^{5}}{\left(1+x^{2}\right)^{3}} d x=-\frac{x^{4}}{4\left(1+x^{2}\right)^{2}}-\frac{x^{2}}{2\left(1+x^{2}\right)}+\frac{1}{2} \lg \left(1+x^{2}\right)$.

Die nämliche Aufgabe kann auch so behandelt werden:

$$
\begin{aligned}
& \frac{x^{5}}{\left(1+x^{2}\right)^{3}}=\frac{x^{3}\left(1+x^{2}\right)-x^{3}}{\left(1+x^{2}\right)^{3}}=\frac{x^{3}}{\left(1+x^{2}\right)^{2}}-\frac{x^{3}}{\left(1+x^{2}\right)^{3}} \\
& \frac{x^{3}}{\left(1+x^{2}\right)^{2}}=\frac{x\left(1+x^{2}\right)-x}{\left(1+x^{2}\right)^{2}}=\frac{x}{1+x^{2}}-\frac{x}{\left(1+x^{2}\right)^{2}} \\
& \frac{x^{3}}{\left(1+x^{2}\right)^{3}}=\frac{x\left(1+x^{2}\right)-x}{\left(1+x^{2}\right)^{3}}=\frac{x}{\left(1+x^{2}\right)^{2}}-\frac{x}{\left(1+x^{2}\right)^{3}} \\
& \frac{x^{5}}{\left(1+x^{2}\right)^{3}}=\frac{x}{\left(1+x^{2}\right)^{3}}-\frac{2 x}{\left(1+x^{2}\right)^{2}}+\frac{x}{x^{2}+1} .
\end{aligned}
$$

121) $\int \frac{x^{5}}{\left(1+x^{2}\right)^{3}} d x=-\frac{1}{4\left(1+x^{2}\right)^{2}}+\frac{1}{1+x^{2}}+\frac{1}{2} \lg \left(x^{2}+1\right)$.

Die Formeln 117) und 119) sind insofern nicht praktisch, als in der ersten bei gleichem Vorzeichen von $m$ und $n$, in der zweiten bei verschiedenen Vorzeichen der Integrand des Integrals rechts nicht direkt vereinfacht ist. Wir. verweisen deshalb auf die Formeln 139)-142), bei welohen dieser Übelstand vermieden ist.

$$
\int \frac{\varphi(x)}{x^{n}-1} d x
$$

Nach der Formel von Moivre (Diff. R. Aufgabe 430) ist

$$
\sqrt[n]{\cos \varphi+i \sin \varphi}=\cos \frac{\varphi}{n}+i \sin \frac{\varphi}{n} .
$$

Soll die Gleichung $x^{n}-1=0$ gelöst, oder $x=\sqrt[n]{1}$ nach dieser Formel gefunden werden, so setzt man $1=\cos 2 k \pi$ $+i \sin 2 k \pi$, indem man $k$ eine beliebige ganze Zahl bedeuten lässt. Es ist dann:

$$
\sqrt[n]{1}=\cos \frac{2 k \pi}{n}+i \sin \frac{2 k \pi}{n}
$$

und für jeden beliebigen Wert von $k$ geht aus der Formel einer der verschiedenen Werte von $\sqrt[n]{1}$ hervor:

$$
\begin{array}{ll}
k=0 ; & w_{0}=\cos 0+i \sin 0=1 \\
k=1 ; & w_{1}=\cos \frac{2 \pi}{n}+i \sin \frac{2 \pi}{n}
\end{array}
$$




$$
\begin{aligned}
& k=2 ; \quad w_{2}=\cos \frac{4 \pi}{n}+i \sin \frac{4 \pi}{n} \\
& k . \quad . \quad . \quad . \quad . \quad . \quad . \quad . \quad .
\end{aligned}
$$

Wird mit der Wahl der ganzen Zahl $k$ in dieser Weise fortgefahren, so wiederholen sich nur die bereits gefundenen Wurzelwerte. So findet man z. B. für $k=n$ :

$w_{n}=\cos \frac{2 n \pi}{n}+i \sin \frac{2 n \pi}{n}=\cos 2 \pi+i \sin 2 \pi$

$$
=\cos 0+i \sin 0=v_{0} .
$$

Weiter ist nach dem Satze von Moivre:

$$
\begin{aligned}
& w_{1}^{2}=\left(\cos \frac{2 \pi}{n}+i \sin \frac{2 \pi}{n}\right)^{2}=\cos \frac{4 \pi}{n}+i \sin \frac{4 \pi}{n}=w_{2} \\
& w_{1}^{3}=\left(\cos \frac{2 \pi}{n}+i \sin \frac{2 \pi}{n}\right)^{3}=\cos \frac{6 \pi}{n}+i \sin \frac{6 \pi}{n}=w_{3}, \text { usw. }
\end{aligned}
$$

Hiernach ist: $w_{0}=1 ; w_{2}=w_{1}^{2} ; \quad w_{3}=w_{1}^{3}, \ldots w_{n-1}=w_{1}^{n-1}$. Es ist auch $\cos \frac{n-k}{n} 2 \pi+i \sin \frac{n-k}{n} 2 \pi=\cos \left(2 \pi-\frac{2 k \pi}{n}\right)$ $+i \sin \left(2 \pi-\frac{2 k \pi}{n}\right)=\cos \frac{2 k \pi}{n}-i \sin \frac{2 k \pi}{n}$, oder es sind die beiden Wurzeln $w_{k}$ und $w_{n-k}$ konjugiert komplex. Ist $n$ gerade, so ist $w_{\frac{n}{2}}$ sich selbst konjugiert und also reell, so dass die beiden reellen Wurzeln \pm 1 existieren. So finden wir $\mathrm{z} . \mathrm{B}$. für $w=\sqrt[6]{1}$, dass $w_{1}=\cos \frac{\pi}{3}+i \sin \frac{\pi}{3}$ und $w_{5}=\cos \frac{5 \pi}{3}$ $+i \sin \frac{5 \pi}{3}=\cos \left(-\frac{\pi}{3}\right)+i \sin \left(-\frac{\pi}{3}\right)=\cos \frac{\pi}{3}-i \sin \frac{\pi}{3}$, also $w_{5}$ konjugiert $\mathrm{zu} w_{1}$ ist.

1. Beispiel: $x=\sqrt[3]{1} ; \quad w_{1}=\cos \frac{2 \pi}{3}+i \sin \frac{2 \pi}{3}=-\frac{1}{2}$ $+\frac{i}{2} \sqrt{3} ; w_{2}=\cos \frac{4 \pi}{3}+i \sin \frac{4 \pi}{3}=-\frac{1}{2}-\frac{i}{2} \sqrt{3}=w_{1}^{2} ;$ $w_{3}=\cos 2 \pi+i \sin 2 \pi=\cos 0+i \sin 0=w_{0}=1$. 
2. Beispiel: $x=\sqrt[4]{1} ; \quad w_{0}=1 ; \quad w_{1}=i$; $w_{2}=w_{1}^{2}=-1 ; \quad w_{3}=w_{1}^{3}=-i$.

3. Beispiel: $x=\sqrt[6]{1} ; \quad w_{0}=1 ; \quad w_{1}=\frac{1}{2}+\frac{i}{2} \sqrt{3}$;

$w_{2}=-\frac{1}{2}+\frac{i}{2} \sqrt{3} ; \quad w_{3}=-1 ; \quad w_{4}=-\frac{1}{2}-\frac{i}{2} \sqrt{3} ;$

$w_{5}=\frac{1}{2}-\frac{i}{2} \sqrt{3}$. Konjugiert ist $w_{5}$ mit $w_{1}, w_{4}$ mit $w_{2}$ und $w_{3}$ mit sich selbst.

Der grösseren Einfachheit wegen wollen wir $w_{1}=\varepsilon$ setzen und können dann die tibrigen Werte von $\sqrt[n]{1}$ durch $\varepsilon^{2}, \varepsilon^{8}, \varepsilon^{4}, \ldots \varepsilon^{n-1}, \varepsilon^{n}=1$ bezeichnen.

Es ist dann:

$\frac{\varphi(x)}{x^{n}-1}=\frac{A_{0}}{x-1}+\frac{A_{1}}{x-\varepsilon}+\frac{A_{2}}{x-\varepsilon^{2}}+\ldots+\frac{A_{n-1}}{x-\varepsilon^{n-1}}$ und nach Formel (8), S. 112:

$$
A_{k}=\left[\frac{\varphi(x)}{n x^{n-1}}\right]_{x=\varepsilon^{k}}=\left[\frac{x \varphi(x)}{n x^{n}}\right]_{x=\varepsilon^{k}}=\frac{\varepsilon^{k} \varphi\left(\varepsilon^{k}\right)}{n} .
$$

$\frac{\varphi(x)}{x^{n}-1}=\frac{1}{n}\left[\frac{\varphi(1)}{x-1}+\frac{\varepsilon \varphi(\varepsilon)}{x-\varepsilon}+\frac{\varepsilon^{2} \varphi\left(\varepsilon^{2}\right)}{x-\varepsilon^{2}}+\ldots+\frac{\varepsilon^{n-1} \varphi\left(\varepsilon^{n-1}\right)}{x-\varepsilon^{n-1}}\right]$.

122) $\int \frac{\varphi(x)}{x^{n}-1} d x=\frac{1}{n}[\varphi(1) \lg (x-1)+\varepsilon \varphi(\varepsilon) \lg (x-\varepsilon)$

$\left.+\ldots+\varepsilon^{n-1} \varphi\left(\varepsilon^{n-1}\right) \lg \left(x-\varepsilon^{n-1}\right)\right]$

Die Glieder dieser Formel können paarweise zu reellen Ausdrücken vereinigt werden. Da nämlich die Wurzelwerte $\varepsilon^{k}$ und $\varepsilon^{n-k}$ konjugiert sind, so mtissen es auch die Zähler $A_{k}=\varepsilon^{k} \varphi\left(\varepsilon^{k}\right)$ und $A_{n-k}=\varepsilon^{n-k} \varphi\left(\varepsilon^{n-k}\right)$ sein, oder es muss $A_{n-k}=A-B i$ sein, wenn $A_{k}=A+B i$ ist. Mithin können wir, da $\left(x-\varepsilon^{k}\right)$ $\left(x-\varepsilon^{n-k}\right)=x^{2}-2 x \cos \frac{2 k \pi}{n}+1$ ist, nach (9), S. 125, setzen: $\varepsilon^{k} \varphi\left(\varepsilon^{k}\right) \lg \left(x-\varepsilon^{k}\right)+\varepsilon^{n-k} \varphi\left(\varepsilon^{n-k}\right) \lg \left(x-\varepsilon^{n-k}\right)=$ $A \lg \left(x^{2}-2 x \cos \frac{2 k \pi}{n}+1\right)-2 B \operatorname{arctg} \frac{x-\cos \frac{2 k \pi}{n}}{2 k \pi}$. $\sin \frac{2 k \pi}{n}$ 
123) $\int \frac{1}{x^{3}-1} d x=\frac{1}{3}\left[\lg (x-1)+\left(-\frac{1}{2}+\frac{i}{2} \sqrt{3}\right) \lg \left(x+\frac{1}{2}-\frac{i}{2} \sqrt{3}\right)\right.$

$$
\left.+\left(-\frac{1}{2}-\frac{i}{2} \sqrt{3}\right) \lg \left(x+\frac{1}{2}+\frac{i}{2} \sqrt{3}\right)\right]
$$

$=\frac{1}{3}\left[\lg (x-1)-\frac{1}{2} \lg \left(x^{2}+x+1\right)-\sqrt{3} \operatorname{arctg} \frac{2 x+1}{\sqrt{3}}\right]$

124) $\int \frac{x^{2}-3 x}{x^{4}-1} d x=\frac{3}{4} \lg \frac{x^{2}+1}{x^{2}-1}+\frac{1}{4} \lg \frac{x-1}{x+1}+\frac{1}{2} \operatorname{arctg} x$.

Man setze in der folgenden Aufgabe $\sqrt{3}=r$.

125) $\int \frac{x}{x^{6}-1} d x$

$=\frac{1}{6}\left[\begin{array}{c}\lg (x-1)+\frac{-1+r i}{2} \lg \left(x-\frac{1+r i}{2}\right)-\frac{1+r i}{2} \lg \left(x+\frac{1-r i}{2}\right) \\ +\lg (x+1)+\frac{-1+r i}{2} \lg \left(x+\frac{1+r i}{2}\right) \\ -\frac{1+r i}{2} \lg \left(x-\frac{1-r i}{2}\right)\end{array}\right\}$

$=\frac{1}{6}\left[\lg \left(x^{2}-1\right)-\frac{1}{2} \lg \left(x^{4}+x^{2}+1\right)-\sqrt{3} \operatorname{arctg} \frac{2 x-1}{\sqrt{3}}\right.$

$\left.+\sqrt{3} \operatorname{arctg} \frac{2 x+1}{\sqrt{3}}\right]$

Die Ausrechnung solcher Integrale ist oft leichter, wenn man die als konjugiert bezeichneten Glieder schon bei der Zerlegung paarweise zusammen fasst. So ist z. B.:

$$
\frac{a x^{2}+b x+c}{x^{3}-1}=\frac{A}{x-1}+\frac{m x+n}{x^{2}+x+1} .
$$

$A=\frac{\varphi(1)}{f^{\prime}(1)}=\frac{a+b+c}{3}$. Wird der Wert für $A$ substituiert, die rechte Seite gleichnamig gemacht und dann die Zähler gleich gesetzt, so ergiebt sich daraus nach dem Satze von den unbestimmten Koefficienten: $m=\frac{2 a-b-c}{3}, n=\frac{a+b-2 c}{3}$. 126) $\int \frac{a x^{2}+b x+c}{x^{3}-1} d x=\frac{1}{3}[(a+b+c) \lg (x-1)$ $\left.+\frac{1}{2}(2 a-b-c) \lg \left(x^{2}+x+1\right)+(b-c) \sqrt{3} \operatorname{arctg} \frac{2 x+1}{\sqrt{3}}\right]$ 
127)

$$
\begin{gathered}
\int \frac{a x^{3}+b x^{2}+c x+d}{x^{4}-1} d x=\frac{a+c}{4} \lg \left(x^{2}-1\right)+\frac{a-c}{4} \lg \left(x^{2}+1\right) \\
+\frac{b+d}{4} \lg \frac{x-1}{x+1}+\frac{b-d}{2} \operatorname{arctg} x \\
\int \frac{\varphi(x)}{x^{n}+1} d x .
\end{gathered}
$$

Die Gleichung: $x^{n}+1=0$, oder $x=\sqrt[n]{-1}$ wird wieder mit Hilfe der Moivre'schen Formel gelöst, indem man setzt: $-1=\cos (1+2 k) \pi+i \sin (1+2 k) \pi$, wenn $k$ irgend eine ganze Zahl bedeutet. Dann ist:

$$
\begin{aligned}
& \sqrt[n]{-1}=\cos \frac{1+2 k}{n} \pi+i \sin \frac{1+2 k}{n} \pi \\
& k=0 ; \quad w_{0} \quad=\cos \frac{\pi}{n}+i \sin \frac{\pi}{n} \\
& k=1 ; \quad w_{1} \quad=\cos \frac{3 \pi}{n}+i \sin \frac{3 \pi}{n} \\
& k=2 ; \quad w_{2} \quad=\cos \frac{5 \pi}{n}+i \sin \frac{5 \pi}{n} \\
& k=n-1 ; \quad w_{n-1}=\cos \frac{2 n-1}{n} \pi+i \sin \frac{2 n-1}{n} \pi .
\end{aligned}
$$

Es kann leicht nachgewiesen werden, dass jede andere ganze Zahl $k$ einen Wurzelwert $w$ hervorbringt, welcher mit einem der vorhergehenden zusammenfällt, so dass im Ganzen nur $n$ unter sich verschiedene Werte möglich sind. Nach dem öfter angeführten Satze von Moivre ist

$$
\begin{gathered}
w_{0}^{3} \quad=\cos \frac{3 \pi}{n}+i \sin \frac{3 \pi}{n}=w_{1} \\
w_{0}^{5} \quad=\cos \frac{5 \pi}{n}+i \sin \frac{5 \pi}{n}=w_{2} \\
. \quad \cdot . \cdot . \\
w_{0}^{2 n-1}=\cos \frac{2 n-1}{n} \pi+i \sin \frac{2 n-1}{n} \pi=w_{n-1} .
\end{gathered}
$$


Auch diese Wurzelwerte sind paarweise konjugiert complex, denn es ist

$$
\begin{aligned}
w_{n-1-k} & =\cos \frac{2 n-2 k-1}{n} \pi+i \sin \frac{2 n-2 k-1}{n} \pi \\
& =\cos \left(2 \pi-\frac{2 k+1}{n} \pi\right)+i \sin \left(2 \pi-\frac{2 k+1}{n} \pi\right) \\
& =\cos \frac{2 k+1}{n} \pi-i \sin \frac{2 k+1}{n} \pi
\end{aligned}
$$

ein Wert, welcher zu $w_{k}$ konjugiert ist.

1. Beispiel: $x=\sqrt[3]{-1} ; \quad w_{0}=\frac{1}{2}+\frac{i}{2} \sqrt{3}, w_{1}=-1$, $w_{2}=\frac{1}{2}-\frac{i}{2} \sqrt{3}$.

2. Beispiel: $x=\sqrt[4]{-1} ; \quad w_{0}=\sqrt{\frac{1}{2}}+i \sqrt{\frac{1}{2}}$, $w_{1}=-\sqrt{\frac{1}{2}}+i \sqrt{\frac{1}{2}}, \quad w_{2}=-\sqrt{\frac{1}{2}}-i \sqrt{\frac{1}{2}}, \quad w_{3}=\sqrt{\frac{1}{2}}-i \sqrt{\frac{1}{2}}$. Es ist $w_{0}$ zu $w_{3}$ und $w_{1} \mathrm{zu} \quad w_{2}$ konjugiert.

Um das vorgelegte Integral auszuführen, setzen wir $w_{0}=\varepsilon$ und

$$
\frac{\varphi(x)}{x^{n}+1}=\frac{A_{1}}{x-\varepsilon}+\frac{A_{2}}{x-\varepsilon^{3}}+\frac{A_{3}}{x-\varepsilon^{5}}+\ldots+\frac{A_{n}}{x-\varepsilon^{2 n-1}}
$$

$A_{k}=\left[\frac{\varphi(x)}{n x^{n-1}}\right]_{x=\varepsilon^{2 k-1}}=\left[\frac{x \varphi(x)}{n x^{n}}\right]_{x=\varepsilon^{2 k-1}}=-\frac{1}{n} \varepsilon^{2 k-1} \varphi\left(\varepsilon^{2 k-1}\right)$.

$$
\frac{\varphi(x)}{x^{n}+1}=-\frac{1}{n}\left[\frac{\varepsilon \varphi(\varepsilon)}{x-\varepsilon}+\frac{\varepsilon^{3} \varphi\left(\varepsilon^{3}\right)}{x-\varepsilon^{3}}+\ldots+\frac{\varepsilon^{2 n-1} \varphi\left(\varepsilon^{2 n-1}\right)}{x-\varepsilon^{2 n-1}}\right] \text {. }
$$

128) $\int \frac{\varphi(x)}{x^{n}+1} d x$

$=-\frac{1}{n}\left[\varepsilon \varphi(\varepsilon) \lg (x-\varepsilon)+\varepsilon^{3} \varphi\left(\varepsilon^{3}\right) \lg \left(x-\varepsilon^{3}\right)+\ldots \varepsilon^{2 n-1} \varphi\left(\varepsilon^{2 n-1}\right) \lg \left(x-\varepsilon^{2 n-1}\right)\right]$.

Ist $\varepsilon^{k} \varphi\left(\varepsilon^{k}\right)=A+B i$, so ist ' der konjugierte Wert $\varepsilon^{2 n-k} \varphi\left(\varepsilon^{2 n-k}\right)=A-B i$, und die entsprechenden Glieder des Integrales lassen sich wiederum zu reellen Werten vereinigen. 
$(A+B i) \lg \left(x-\cos \frac{2 k+1}{n} \pi-i \sin \frac{2 k+1}{n} \pi\right)$

$+(A-B i) \lg \left(x-\cos \frac{2 k+1}{n} \pi+i \sin \frac{2 k+1}{n} \pi\right)$

$=A \lg \left(x^{2}-2 x \cos \frac{2 k+1}{n} \pi+1\right)-2 B \operatorname{arctg} \frac{x-\cos \frac{2 k+1}{n} \pi}{\sin \frac{2 k+1}{n} \pi}$.

129) $\int \frac{2 x^{2}-x+3}{x^{3}+1} d x=2 \lg (x+1)+\frac{2}{\sqrt{3}} \operatorname{arctg} \frac{2 x-1}{\sqrt{3}}$

130) $\int \frac{x^{2}}{x^{4}+1} d x=-\frac{\sqrt{2}}{4}\left[\frac{1}{2} \lg \frac{x^{2}+x \sqrt{2}+1}{x^{2}-x \sqrt{2}+1}\right.$

$-\operatorname{arctg}(x \sqrt{2}-1)-\operatorname{arctg}(x \sqrt{2}+1)]$

131) $\int \frac{x^{5}+x^{2}+4}{x^{6}+1} d x=\frac{1}{6}\left[\lg \left(x^{6}+1\right)-2 \sqrt{3} \lg \frac{(2 x-\sqrt{3})^{2}+1}{(2 x+\sqrt{3})^{2}+1}\right.$

$\left.+6 \operatorname{arctg} \frac{x^{3}-2 x}{2 x^{2}-1}\right]$

132) $\int \frac{a x^{2}+b x+c}{x^{3}+1} d x=\frac{1}{3}[(a-b+c) \lg (x+1)$

$\left.+\frac{1}{2}(2 a+b-c) \lg \left(x^{2}-x+1\right)+(b+c) \sqrt{3} \operatorname{arctg} \frac{2 x-1}{\sqrt{3}}\right]$

133) $\int \frac{1}{x^{6}-1} d x=\frac{1}{2} \int \frac{1}{x^{3}-1} d x-\frac{1}{2} \int \frac{1}{x^{3}+1} d x$

$=\frac{1}{6}\left[\lg \frac{x-1}{x+1}+\frac{1}{2} \lg \frac{x^{2}-x+1}{x^{2}+x+1}-\sqrt{3} \operatorname{arctg} \frac{2 x+1}{\sqrt{3}}-\sqrt{3} \operatorname{arctg} \frac{2 x-1}{\sqrt{3}}\right]$

134) $\int \frac{1}{x^{4}+1} d x=\frac{1}{4 \sqrt{2}} \lg \frac{x^{2}+x \sqrt{2}+1}{x^{2}-x \sqrt{2}+1}+\frac{1}{2 \sqrt{2}} \operatorname{arctg} \frac{x \sqrt{2}}{1-x^{2}}$

135) $\int \frac{x^{5}}{\left(x^{3}+1\right)^{2}} d x=\frac{1}{3} \lg \left(x^{3}+1\right)+\frac{1}{3\left(x^{3}+1\right)}$. 


\section{\& 3. Reduktionsformeln.}

Verwickelte Integrale können oft durch Reduktionsformeln (vgl. S. 131) auf einfachere Formen gebracht werden, und wir wollen einige dazu dienliche Formeln jetzt aufstellen.

Reduktionsformeln fur: $\int \frac{1}{\left(a+b x+c x^{2}\right)^{n}} d x$. Setzt man $a+b x+c x^{2}=t$, so ist:

$$
\frac{d}{d x}\left(\frac{b+2 c x}{t^{n}}\right)=\frac{2 c}{t^{n}}-\frac{n(b+2 c x)^{2}}{t^{n+1}} .
$$

Da $(b+2 c x)^{2}$ durch $4 c t-\left(4 a c-b^{2}\right)$ ersetzt werden kann, so ist auch:

$$
\frac{d}{d x}\left(\frac{b+2 c x}{t^{n}}\right)=\frac{\left(4 a c-b^{2}\right) n}{t^{n+1}}-\frac{2 c(2 n-1)}{t^{n}} .
$$

Wird diese Gleichung Glied für Glied integriert, und $n-1$ statt $n$ gesetzt, so kann ihr folgende Form gegeben werden: 136) $\int \frac{1}{t^{n}} d x=\frac{b+2 c x}{(n-1)\left(4 a c-b^{2}\right) t^{n-1}}+\frac{(2 n-3) 2 c}{(n-1)\left(4 a c-b^{2}\right)} \int \frac{1}{t^{n-1}} d x$. Furr $b=0$ und $c=1$ entsteht die speciellere Formel:

$$
\begin{aligned}
\int \frac{1}{\left(x^{2}+a\right)^{n}} d x=\frac{1}{2(n-1) a} & \cdot \frac{x}{\left(x^{2}+a\right)^{n-1}} \\
& +\frac{2 n-3}{2(n-1) a} \int \frac{1}{\left(x^{2}+a\right)^{n-1}} d x
\end{aligned}
$$

Behält $t$ seine Bedeutung, so ist:

$$
\begin{aligned}
& \frac{d}{d x}\left(\frac{x^{m-1}}{t^{n}}\right)=(m-1) \frac{x^{m-2}}{t^{n}}-\frac{n x^{m-1}(b+2 c x)}{t^{n+1}} \\
& =\frac{(m-1) x^{m-2} t-n x^{m-1}(b+2 c x)}{t^{n+1}}
\end{aligned}
$$

Wird wieder Glied für Glied integriert, so kann die Gleichung in folgende Form gebracht werden:

138) $\int \frac{x^{m}}{t^{n+1}} d x=-\frac{1}{(2 n-m+1) c} \cdot \frac{x^{m-1}}{t^{n}}$

$$
-\frac{(n-m+1) b}{(2 n-m+1) c} \int \frac{x^{m-1}}{t^{n+1}} d x+\frac{(m-1) a}{(2 n-m+1) c} \int \frac{x^{m-2}}{t^{n+1}} d x .
$$


Wie wir sehen, zerlegt diese Formel das vorgelegte Integral in einen fertig gestellten Teil und zwei noch auszuführende Integrale, bei welchen der Nenner $t^{n+1}$ unverändert geblieben, der Exponent des Zählers aber um 1 und 2 kleiner geworden ist. Die Formel kommt in Anwendung, wenn $m$ positiv ist. Sie lässt sich aber auch leicht so umsetzen, dass das letzte Integral rechts zur Aufgabe wird. Nach dieser Umformung ist sie brauchbar für ein negatives $m$. Gewöhnlich wird aber in diesem Falle durch die Substitution $x=\frac{1}{z}$ das Integral transformiert, oder vor der Integration die Zerlegung in Partialbrïche ausgeführt.

Die Reduktionsformeln für $\int x^{n}\left(a+b x^{m}\right)^{p} d x$.

$$
\begin{aligned}
\frac{d}{d x}\left[x^{n}\left(a+b x^{m}\right)^{p}\right] & =n x^{n-1}\left(a+b x^{m}\right)^{p}+m b p\left(a+b x^{m}\right)^{p-1} x^{m+n-1} . \\
x^{n-1}\left(a+b x^{m}\right)^{p} & =a x^{n-1}\left(a+b x^{m}\right)^{p-1}+b x^{m+n-1}\left(a+b x^{m}\right)^{p-1}
\end{aligned}
$$

ist eine identische Gleichung, durch deren Vermittelung man erhält:

$$
\begin{aligned}
\frac{d}{d x}\left[x^{n}\left(a+b x^{m}\right)^{p}\right]= & n a x^{n-1}\left(a+b x^{m}\right)^{p-1} \\
& +b(m p+n) x^{m+n-1}\left(a+b x^{m}\right)^{p-1} .
\end{aligned}
$$

Das Integral dieser Gleichung kommt in 2 verschiedenen Formen zur Anwendung:

$$
\begin{array}{rl}
\int x^{n-1}\left(a+b x^{m}\right)^{p-1} & d x=\frac{x^{n}\left(a+b x^{m}\right)^{p}}{n a} \\
& -\frac{b(m p+n)}{n a} \int x^{m+n-1}\left(a+b x^{m}\right)^{p-1} d x
\end{array}
$$

140) $\int x^{m+n-1}\left(a+b x^{m}\right)^{p-1} d x=\frac{x^{n}\left(a+b x^{m}\right)^{p}}{b(m p+n)}$

$$
-\frac{n a}{b(m p+n)} \int x^{n-1}\left(a+b x^{m}\right)^{p-1} d x .
$$

Je nach der Absicht, den Exponenten von $x$ um $m$ Einheiten zu vergrössern oder zu verkleinern, muss die eine oder die andere Formel Anwendung finden. Die Potenz des Binoms bleibt unverändert. 
$\frac{d}{d x}\left[x^{n}\left(a+b x^{m}\right)^{p}\right]=n x^{n-1}\left(a+b x^{m}\right)^{p}$

$+b m p x^{m+n-1}\left(a+b x^{m}\right)^{p-1}$.

$$
b x^{m+n-1}=x^{n-1}\left(a+b x^{m}\right)-a x^{n-1} ;
$$

$\frac{d}{d x}\left[x^{n}\left(a+b x^{m}\right)^{p}\right]=(m p+n) x^{n-1}\left(a+b x^{m}\right)^{p}$

$$
\text { - } a m p x^{n-1}\left(a+b x^{m}\right)^{p-1} \text {. }
$$

Aus den Integralwerten dieser Gleichung lassen sich die folgenden Formeln zusammensetzen:

141) $\int x^{n-1}\left(a+b x^{m}\right)^{p} d x=\frac{x^{n}\left(a+b x^{m}\right)^{p}}{m p+n}$

$$
+\frac{a m p}{m p+n} \int x^{n-1}\left(a+b x^{m}\right)^{p-1} d x
$$

142) $\int x^{n-1}\left(a+b x^{m}\right)^{p-1} d x=$

$$
\begin{aligned}
& =-\frac{x^{n}\left(a+b x^{m}\right)^{p}}{a m p} \\
& +\frac{m p+n}{a m p} \int x^{n-1}\left(a+b x^{m}\right)^{p} d x .
\end{aligned}
$$

Während die zweite Formel dazu dient, den Exponenten $p$ um 1 zu vergrössern, wird derselbe durch die erste um 1 verkleinert.

§4. Irrationale algebraisehe Funktionen.

$$
\int f(x, \sqrt[n]{a+b x}) d x .
$$

Ein solehes Integral wird rational durch die Substitution:

$$
\sqrt[n]{a+b x}=z, \quad x=\frac{z^{n}-a}{b}, \quad d x=\frac{n z^{n-1}}{b} d z .
$$

Beispiel: $S=\int x \sqrt{a+b x} d x ; \quad a+b x=z^{2}, \quad x=\frac{z^{2}-a}{b}$,

$$
d x=\frac{2 z}{b} d z ; \quad S=\frac{2}{b^{2}} \int\left(z^{4}-a z^{2}\right) d z=\frac{2}{b^{2}}\left(\frac{z^{5}}{5}-\frac{a z^{3}}{3}\right) .
$$

143) $\int x \sqrt{a+b x} d x=\frac{2}{b^{2}} \sqrt{(a+b x)^{3}}\left(\frac{a+b x}{5}-\frac{a}{3}\right)$.

Das Integral kann auch mittelst der Integration nach Teilen ausgeführt werden:

$$
\begin{aligned}
\int x(a+b x)^{\frac{1}{2}} d x & =\frac{2(a+b x)^{\frac{3}{2}} x}{3 b}-\frac{2}{3 b} \int(a+b x)^{\frac{3}{2}} d x \\
& =\frac{2(a+b x)^{\frac{3}{2}} x}{3 b}-\frac{2}{3 b} \cdot \frac{2}{5 b}(a+b x)^{\frac{5}{2}}
\end{aligned}
$$


144) $\int x(a+b x)^{\frac{1}{2}} d x=\frac{2}{5 b^{2}} \sqrt{(a+b x)^{3}}\left(b x-\frac{2 a}{3}\right)$

145) $\int \frac{x^{2}}{\sqrt[3]{1+x}} d x=3 \int\left(z^{3}-1\right)^{2} z d z=3\left(\frac{z^{8}}{8}-\frac{2 z^{5}}{5}+\frac{z^{2}}{2}\right)$

$$
=3 \sqrt[3]{(1+x)^{2}}\left(\frac{1}{8}(1+x)^{2}-\frac{2}{5}(1+x)+\frac{1}{2}\right)
$$

146) $\int \frac{1}{\sqrt{a+b x}} d x=\frac{2}{b} \sqrt{a+b x}$

147) $\int \frac{1}{\sqrt[n]{(a+b x)^{p}}} d x=\frac{n}{(n-p) b \sqrt[n]{(a+b x)^{p-n}}}$

148) $\int \frac{1}{\sqrt[3]{a+b x}} d x=\frac{3}{2 b} \sqrt[3]{(a+b x)^{2}}$

149) $\int x \sqrt[3]{a+b x} d x=\frac{3}{28 b^{2}}(4 b x-3 a) \sqrt[3]{(a+b x)^{4}}$

150) $\int x^{2} \sqrt[3]{a+b x} d x=\frac{3}{b^{8}}\left[\frac{(a+b x)^{8}}{10}-\frac{2 a}{7}(a+b x)^{2}\right.$

$$
\left.+\frac{a^{2}}{4}(a+b x)\right] \sqrt[3]{a+b x}
$$

151) $\int \frac{x}{\sqrt[4]{a+b x}} d x=\frac{4}{21 b^{2}}(3 b x-4 a) \sqrt[4]{(a+b x)^{3}}$

152) $\int x \sqrt[3]{x-4} d x=\frac{3}{7}\left(x^{2}-x-12\right) \sqrt[3]{x-4}$

153) $\int \frac{x^{2}}{3 \sqrt[3]{x+2}} d x=\left(\frac{x^{2}}{8}+\frac{9-3 x}{10}\right) \sqrt[3]{(x+2)^{2}}$

154) $\int \frac{x^{2}}{\sqrt{3 x+5}} d x=\frac{2}{27}\left(\frac{9 x^{2}}{5}-4 x+\frac{40}{3}\right) \sqrt{5+3 x}$

155) $\int \frac{\sqrt{x}}{x-1} d x=2 \sqrt{x}+\lg \frac{\sqrt{x}-1}{\sqrt{x}+1}$

156) $\int \frac{1}{x \sqrt{a+x}} d x=\frac{1}{\sqrt{a}} \lg \frac{\sqrt{x+a}-\sqrt{a}}{\sqrt{x+a}+\sqrt{a}}$

157) $\int \frac{1}{x \sqrt{x-a}} d x=\frac{2}{\sqrt{a}} \operatorname{arctg} \sqrt{\frac{x-a}{a}}$

158) $\int \frac{1}{(x+1) \sqrt{1-x}} d x=\frac{1}{\sqrt{2}} \lg \frac{\sqrt{1-x}-\sqrt{2}}{\sqrt{1-x}+\sqrt{2}}$ 
159) $\int \frac{1}{x \sqrt{a+b x}} d x=\frac{1}{\sqrt{a}} \lg \frac{\sqrt{a+b x}-\sqrt{a}}{\sqrt{a+b x}+\sqrt{a}} ; \quad a>0$

160) $\int \frac{1+\sqrt{x}-\sqrt[3]{x^{2}}}{1+\sqrt[3]{x}} d x=-\frac{3}{4} x^{\frac{4}{3}}+\frac{6}{7} x^{\frac{7}{6}}+x-\frac{6}{5} x^{\frac{8}{6}}$ $+2 x^{\frac{1}{2}}-6 x^{\frac{1}{6}}+6 \operatorname{arctg} \sqrt[6]{x}$

161) $\int \frac{x}{\sqrt[3]{x+1}-\sqrt{x+1}} d x=-\frac{3}{2}(x+1)^{\frac{2}{3}}-\frac{6}{5}(x+1)^{\frac{5}{8}}$

$$
-(x+1)-\frac{6}{7}(x+1)^{\frac{7}{6}}-\frac{3}{4}(x+1)^{\frac{4}{3}}-\frac{2}{3}(x+1)^{\frac{3}{2}}
$$

Man setze $x+1=z^{6}$.

162) $\int \frac{\sqrt{x+1}}{x} d x=2 \sqrt{1+x}+\lg \frac{\sqrt{x+1}-1}{\sqrt{x+1}+1}$

163) $\begin{aligned} \int \frac{1}{\sqrt{x-a}-\sqrt{x-b}} d x & =\frac{1}{b-a} \int(\sqrt{x-a}+\sqrt{x-b}) d x \\ & =\frac{2}{3(b-a)}\left[(x-a)^{\frac{3}{2}}+(x-b)^{\frac{3}{2}}\right]\end{aligned}$

164) $\int \frac{1}{\sqrt{x+a}+\sqrt{x}} d x=\frac{2}{3 a}\left[(x+a)^{\frac{3}{2}}-x^{\frac{3}{2}}\right]$

165) $\int \frac{1+\sqrt{x}}{1-\sqrt{x}} d x=-(x+4 \sqrt{x}+4 \lg (1-\sqrt{x}))$

166) $\int \frac{1}{x+\sqrt{x}} d x=2 \lg (1+\sqrt{x})$

167) $\int \sqrt{1+\sqrt{x}} d x=4 \sqrt{1+\sqrt{x}}\left[\frac{(1+\sqrt{x})^{2}}{5}-\frac{1+\sqrt{x}}{3}\right]$

168) $\int \frac{1}{\sqrt{1+x}+\sqrt[3]{1+x}} d x=2 \sqrt{1+x}-3 \sqrt[3]{1+x}$

$$
+6 \sqrt[6]{1+x}-6 \lg (1+\sqrt[6]{1+x})
$$

169) $\int \frac{1}{\alpha+\beta \sqrt{a+b x}} d x=\frac{2}{b \beta} \sqrt{a+b x}-\frac{2 \alpha}{b \beta^{2}} \lg (\alpha+\beta \sqrt{a+b x})$

170) $\int \frac{1}{(\alpha+\beta x) \sqrt{a+b x}} d x=2 \int \frac{1}{\alpha b-a \beta+\beta z^{2}} d z$.

Die weitere Ausfuhrung hängt von dem besonderen Werte von $\beta(\alpha b-a \beta)$ ab. Hier ist $z^{2}=a+b x$. 
Bei derartigen Aufgaben kann man zweckmässig vor der Substitution die Integration nach Teilen vornehmen, um dadurch etwas hohe Werte von $n$ zu erniedrigen. Die folgenden Beispiele dienen zur Anleitung.

$$
\int x^{n} \sqrt{a+b x} d x=\frac{2}{3 b}(a+b x)^{\frac{3}{2}} x^{n}-\frac{2 n}{3 b} \int x^{n-1}(a+b x)^{\frac{3}{2}} d x
$$

$\int x^{n} \sqrt[3]{a+b x} d x=\frac{3(a+b x)^{\frac{4}{3}} x^{n}}{4 b}-\frac{3 n}{4 b} \int x^{n-1}(a+b x)^{\frac{4}{3}} d x$

$$
\begin{gathered}
\int x^{n} \frac{1}{\sqrt[3]{a+b x}} d x=\frac{3(a+b x)^{\frac{2}{3}} x^{n}}{2 b}-\frac{3 n}{2 b} \int x^{n-1}(a+b x)^{\frac{2}{3}} d x . \\
\int \frac{A_{n} x^{n}+A_{n-1} x^{n-1}+\ldots+A_{1} x+A_{0}}{\sqrt{a+2 b x+c x^{2}}} d x .
\end{gathered}
$$

Mit Hilfe des Satzes von den unbestimmten Koeffizienten kann ein solehes Integral in einen fertigen Teil und in ein noch auszuführendes Integral zerlegt werden. Wie dies geschieht, wollen wir an einem Beispiele zeigen. Wir setzen:

$$
\begin{aligned}
& \int \frac{30 x^{5}+30 x^{4}+12 x^{3}+21 x^{2}-15 x-1}{\sqrt{4+2 x+3 x^{2}}} d x \\
& =\left(\alpha_{4} x^{4}+\alpha_{3} x^{3}+\alpha_{2} x^{2}+\alpha_{1} x+\alpha_{0}\right) \sqrt{4+2 x+3 x^{2}} \\
& +K \int \frac{1}{\sqrt{4+2 x+3 x^{2}}} d x
\end{aligned}
$$

worin die $\alpha$ und $K$ noch unbestimmte Koeffizienten sind. $\mathrm{Zu}$ dieser identischen Gleichung werden wir durch die Thatsache berechtigt, dass auf beiden Seiten der Form nach völlig gleiche Ausdrïcke entstehen, wenn wir wieder differentiieren. Man erhält dann:

$$
\begin{aligned}
& \frac{30 x^{5}+30 x^{4}+12 x^{3}+21 x^{2}-15 x-1}{\sqrt{4+2 x+3 x^{2}}} \\
& =\left(4 \alpha_{4} x^{3}+3 \alpha_{3} x^{2}+2 \alpha_{2} x+\alpha_{1}\right) \sqrt{4+2 x+3 x^{2}} \\
& \quad+\frac{(1+3 x)\left(\alpha_{4} x^{4}+\alpha_{3} x^{3}+\alpha_{2} x^{2}+\alpha_{1} x+\alpha_{0}\right)+K}{\sqrt{4+2 x+3 x^{2}}} .
\end{aligned}
$$$$
=\left(4 \alpha_{4} x^{8}+3 \alpha_{3} x^{2}+2 \alpha_{2} x+\alpha_{1}\right)\left(3 x^{2}+2 x+4\right)
$$$$
+(1+3 x)\left(\alpha_{4} x^{4}+\alpha_{3} x^{3}+\alpha_{2} x^{2}+\alpha_{1} x+\alpha_{0}\right)+K \text {. }
$$ 
Der Satz von den unbestimmten Koeffizienten führt zu den Werten der $\alpha$. Es ist $30=15 \alpha_{4}, \alpha_{4}=2 ; 30=12 \alpha_{3}+9 \alpha_{4}$, $\alpha_{3}=1 ; 12=9 \alpha_{2}+7 \alpha_{3}+16 \alpha_{4}, \alpha_{2}=-3 ; 21=6 \alpha_{1}+5 \alpha_{2}+12 \alpha_{3}$, $\alpha_{1}=4 ;-15=3 \alpha_{0}+3 \alpha_{1}+8 \alpha_{2}, \alpha_{0}=-1 ;-1=K+4 \alpha_{1}+\alpha_{0}$, $K=-16$.

175) $\int \frac{30 x^{5}+30 x^{4}+12 x^{3}+21 x^{2}-15 x-1}{\sqrt{4+2 x+3 x^{2}}} d x$

$=\left(2 x^{4}+x^{3}-3 x^{2}+4 x-1\right) \sqrt{4+2 x+3 x^{2}}-16 \int \frac{1}{\sqrt{4+2 x+3 x^{2}}} d x$.

Bevor wir uns mit der weiteren Ausführung des übrig gebliebenen Integrales beschäftigen, sollen zur Berechnung des anderen Teiles allgemein gültige Formeln aufgestellt werden, und wir wählen dazu als Zähler eine Funktion von ganz beliebigem Grade. Nur der einfacheren Darstellung wegen nennen wir diesen Grad nicht $n$, sondern wir wählen dafür eine beliebige Zahl, z. B. $n=9$.

$$
\begin{aligned}
& \int \frac{A_{9} x^{9}+A_{8} x^{8}+\ldots+A_{1} x+A_{0}}{\sqrt{a+2 b x+c x^{2}}} d x \\
& =\left(\alpha_{8} x^{8}+\alpha_{7} x^{7}+\ldots+\alpha_{1} x+\alpha_{0}\right) \sqrt{a+2 b x+c x^{2}} \\
& \quad+K \int \frac{1}{\sqrt{a+2 b x+c x^{2}}} d x .
\end{aligned}
$$

Wird diese Gleichung differentiiert und der Nenner durch Multiplikation beseitigt, so erhält man:

$A_{9} x^{9}+A_{8} x^{8}+A_{7} x^{2}+\ldots .+A_{1} x+A_{0}$

$$
\begin{aligned}
& =\left(8 \alpha_{8} x^{7}+7 \alpha_{7} x^{6}+\ldots+\alpha_{1}\right)\left(a+2 b x+c x^{2}\right) \\
& +\left(\alpha_{8} x^{8}+\alpha_{7} x^{7}+\alpha_{6} x^{6}+\ldots+\alpha_{1} x+\alpha_{0}\right)(b+c x)+K
\end{aligned}
$$

und durch Vergleich der Koeffizienten gleich hoher Potenzen von $x$ die folgenden Relationen:

$$
\begin{aligned}
& A_{9}=9 c \alpha_{8} \\
& A_{8}=8 c \alpha_{7}+17 b \alpha_{8} \\
& A_{7}=7 c \alpha_{6}+15 b \alpha_{7}+8 a \alpha_{8} \\
& A_{6}=6 c \alpha_{5}+13 b \alpha_{6}+7 a \alpha_{7} \\
& A_{5}=5 c \alpha_{4}+11 b \alpha_{5}+6 a \alpha_{6} \\
& A_{4}=4 c \alpha_{3}+9 b \alpha_{4}+6 a \alpha_{5}
\end{aligned}
$$

Dölp. Aufgaben. 


$$
\begin{aligned}
& A_{3}=3 c \alpha_{2}+7 b \alpha_{3}+4 a \alpha_{4} \\
& A_{2}=2 c \alpha_{1}+5 b \alpha_{2}+3 a \alpha_{8} \\
& A_{1}=1 c \alpha_{0}+3 b \alpha_{1}+2 a \alpha_{2} \\
& A_{0}=\quad 1 b \alpha_{0}+1 a \alpha_{1}+K
\end{aligned}
$$

Aus diesen sehr übersichtlichen Gleichungen werden die Koeffizienten $\alpha$ und $K$ berechnet. Selbstrerständlich kann unter den $A$ eine Anzahl den Wert Null besitzen.

Die Ermittelung des übrig gebliebenen Integrales ist eine ganz versohiedene, je nachdem $c$ positiv oder negativ ist. Wir untersuchen zuerst:

$$
\begin{gathered}
\int \frac{1}{\sqrt{a+2 b x+c x^{2}}} d x, \text { wenn } c>0, \quad \text { und setzen } \\
\sqrt{a+2 b x+c x^{2}}=x \sqrt{c}+z, \quad x=\frac{z^{2}-a}{2(b-\sqrt{c} z)}, \\
d x=\frac{-\sqrt{c} z^{2}+2 b z-a \sqrt{c}}{2(b-\sqrt{c} z)^{2}} d z=\frac{z+x \sqrt{c}}{b-\sqrt{c} z} d z, \\
\sqrt{a+2 b x+c x^{2}}=\frac{-\sqrt{c} z^{2}+2 b z-a \sqrt{c}}{2(b-\sqrt{c} z)} \\
\int \frac{1}{\sqrt{a+2 b x+c x^{2}}} d x=\int \frac{1}{b-z \sqrt{c}} d z=-\frac{1}{\sqrt{c}} \lg (b-z \sqrt{c}) \\
=-\frac{1}{\sqrt{c}} \lg \left(b+c x-\sqrt{c} \sqrt{a+2 b x+c x^{2}}\right) \\
\text { oder }=\frac{1}{\sqrt{c}} \lg \left(b+c x+\sqrt{c} \sqrt{\left.a+2 b x+c x^{2}\right)},\right.
\end{gathered}
$$

je nachdem $\sqrt{c}$ mit dem + oder - Vorzeichen genommen wird. Dass die beiden Werte, wie es sein muss, nur um eine Konstante, nämlich um $\frac{1}{\sqrt{c}} \lg \left(b^{2}-a c\right)$ differieren, ist leicht
nachzuweisen.

Für $c<0$ treten in dieser Formel imaginäre Werte auf. Statt sie umzuwandeln ist es bequemer, diesen Fall beson. ders zu behandeln. Wir schreiben hier:

$$
\int \frac{1}{\sqrt{a+2 b x-c x^{2}}} d x, \text { wenn } c>0 \text {. }
$$




$$
y=\int \frac{d x}{\sqrt{a+2 b x-c x^{2}}}=\int \frac{\sqrt{c} d x}{\sqrt{\left(b^{2}+a c\right)-(b-c x)^{2}}} .
$$

Man setzt $z=\frac{b-c x}{\sqrt{b^{2}+a c}}, \quad x=\frac{b-z \sqrt{b^{2}+a c}}{c}$,

$d x=-\frac{1}{c} \sqrt{b^{2}+a c} d z, \sqrt{a+2 b x-c x^{2}}=\sqrt{\frac{b^{2}+a c}{c}\left(1-z^{2}\right)}$.

178) $\int \frac{d x}{\sqrt{a+2 b x-c x^{2}}}=-\frac{1}{\sqrt{c}} \int \frac{d z}{\sqrt{1-z^{2}}}=-\frac{1}{\sqrt{c}} \arcsin z$

$$
=-\frac{1}{\sqrt{c}} \arcsin \frac{b-c x}{\sqrt{b^{2}+a c}} .
$$

Die in diesem zweiten Falle benutzte Transformation wird unmöglich, falls $b^{2}+a c=0$ ist. Das kann nur für ein negatives $a$ eintreten, da $c$ positiv sein sollte. Dann geht aber für $\alpha=-a$ der Radikand $-\alpha+2 b x-c x^{2}$ in

$$
-\frac{1}{c}\left[(c x-b)^{2}-\left(b^{2}-a c\right)\right]=-\frac{1}{c}(c x-b)^{2}
$$

uber, so dass $\sqrt{a+2 b x-c x^{2}}=\sqrt{\frac{-1}{c}}(c x-b)$ wird, die Irrationalität also verschwindet und das Integral unter Verwendung imaginärer Grössen nach früheren Vorschriften behandelt werden kann.

In den Resultaten der nachfolgenden Aufgaben ist der in der Aufgabe vorkommende Wurzelwert häufig kurzweg durch $R$ bezeichnet.

$$
\text { 179) } \begin{aligned}
\int \frac{x^{3}+5 x^{2}-3 x+4}{\sqrt{x^{2}+x+1}} d x & =\left(\frac{x^{2}}{3}+\frac{25}{12} x-\frac{163}{24}\right) \sqrt{x^{2}+x+1} \\
& +\frac{85}{16} \lg \left(x+\frac{1}{2}+\sqrt{x^{2}+x+1}\right)
\end{aligned}
$$

180) $\int \frac{4 x^{4}+15 x^{3}-9 x^{2}+14 x+46}{\sqrt{x^{2}+6 x+5}} d x=\left(x^{3}-2 x^{2}+3 x+7\right) R$

181) $\int \frac{5 x^{2}-2 x+10}{\sqrt{3 x^{2}-5 x+8}} d x=\left(\frac{5}{6} x+\frac{17}{12}\right) R$

$$
+\frac{55}{8 \sqrt{3}} \lg \left(3 x-\frac{5}{2}+\sqrt{3} R\right)
$$


182) $\int \frac{x^{3}+4 x^{2}-6 x+3}{\sqrt{5+6 x-x^{2}}} d x=-\left(\frac{x^{2}}{3}+\frac{9 x}{2}+\frac{227}{6}\right) R$

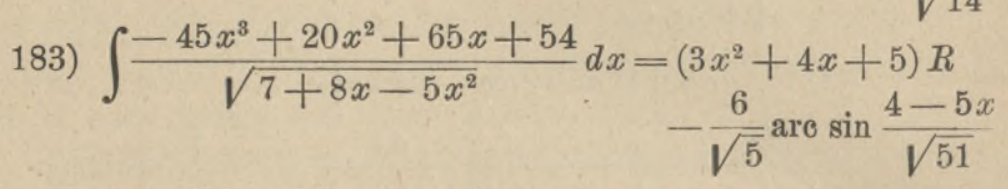
$-139 \arcsin \frac{3-x}{\sqrt{14}}$ 184) $\int \frac{m x^{2}+n x+p}{\sqrt{a+2 b x+c x^{2}}} d x=\left(\frac{m x}{2 c}+\frac{2 c n-3 b m}{2 e^{2}}\right) \sqrt{a+2 b x+c x^{2}}$ $+\left(p-\frac{2 b c n-3 b^{2} m+a c m}{2 c^{2}}\right) \int \frac{1}{\sqrt{a+2 b x+c x^{2}}} d x$

185) $\int \sqrt{a+2 b x+c x^{2}} d x=\int \frac{c x^{2}+2 b x+a}{\sqrt{a+2 b x+c x^{2}}} d x$

$$
=\left(\frac{x}{2}+\frac{b}{2 c}\right) R+\left(\frac{a}{2}-\frac{b^{2}}{2 c}\right) \int \frac{1}{\sqrt{a+2 b x+c x^{2}}} d x
$$

Es wird also z. B.

$$
\begin{aligned}
& \int \sqrt{1+x^{2}} d x=\frac{1}{2} x \sqrt{1+x^{2}}+\frac{1}{2} \lg \left(x+\sqrt{1+x^{2}}\right), \\
& \int \sqrt{1-x^{2}} d x=\frac{1}{2} x \sqrt{1-x^{2}}+\frac{1}{2} \arcsin x .
\end{aligned}
$$

186) $\int \sqrt{3 x^{2}+10 x+9} d x=\left(\frac{x}{2}+\frac{5}{6}\right) R+\frac{1}{\sqrt{27}} \lg (3 x+5+\sqrt{3} R)$ 187) $\int \sqrt{11+12 x-8 x^{2}} d x=\left(\frac{x}{2}-\frac{3}{8}\right) R-\frac{31}{8 \sqrt{2}} \arcsin \frac{3-4 x}{\sqrt{31}}$ 188) $\int x \sqrt{8+x-x^{2}} d x=\int \frac{-x^{3}+x^{2}+8 x}{\sqrt{8+x-x^{2}}} d x$

$$
=\left(\frac{x^{2}}{3}-\frac{x}{12}-\frac{67}{24}\right) R-\frac{33}{16} \arcsin \frac{1-2 x}{\sqrt{33}}
$$

189) $\int \frac{x}{\sqrt{a+2 b x+c x^{2}}} d x=\frac{1}{c} \sqrt{a+2 b x+c x^{2}}$

$$
-\frac{b}{c} \int \frac{1}{\sqrt{a+2 b x+c x^{2}}} d x
$$


190) $\int \frac{x^{2}}{\sqrt{a+2 b x+c x^{2}}} d x=\left(\frac{x}{2 c}-\frac{3 b}{2 c^{2}}\right) R+\frac{3 b^{2}-a c}{2 c^{2}} \int \frac{1}{K} d x$

191) $\int \frac{x^{8}}{\sqrt{a+2 b x+c x^{2}}} d x=\left(\frac{x^{2}}{3 c}-\frac{5 b x}{6 c^{2}}+\frac{5 b^{2}}{2 c^{3}}-\frac{2 a}{3 c^{2}}\right) R$ $+\left(\frac{3 a b}{2 c^{2}}-\frac{5 b^{3}}{2 c^{3}}\right) \int \frac{1}{R} d x$

192) $\int \frac{x^{4}}{\sqrt{3+2 x+x^{2}}} d x=\left(\frac{x^{3}}{4}-\frac{7 x^{2}}{12}+\frac{x}{3}+\frac{5}{2}\right) R$

$$
-\frac{7}{2} \lg (1+x+R)
$$

193) $\int \frac{x^{5}}{\sqrt{1+2 x+3 x^{2}}} d x=\left(\frac{x^{4}}{15}-\frac{x^{3}}{20}+\frac{x^{2}}{108}+\frac{7 x}{405}-\frac{19}{810}\right) R$ $+\frac{1}{162 \sqrt{3}} \lg (1+3 x+\sqrt{3} R)$

194) $\int x \sqrt{a+2 b x+c x^{2}} d x=\int \frac{c x^{3}+2 b x^{2}+a x}{\sqrt{a+2 b x+c x^{2}}} d x$

$$
=\left(\frac{x^{2}}{3}+\frac{b x}{6 c}+\frac{a}{3 c}-\frac{b^{2}}{2 c^{2}}\right) R+\left(\frac{b^{3}}{2 c^{2}}-\frac{a b}{2 c}\right) \int \frac{1}{R} d x
$$

195) $\int \sqrt{\left(5 x^{2}+4 x+3\right)^{3}} d x=\int \frac{25 x^{4}+40 x^{3}+46 x^{2}+24 x+9}{\sqrt{5 x^{2}+4 x+3}} d x$ $=\left(\frac{5 x^{3}}{4}+\frac{3 x^{2}}{2}+\frac{79 x}{40}+\frac{63}{100}\right) R+\frac{363}{200 \sqrt{5}} \lg (2+5 x+\sqrt{5} R)$

196) $\int(2 x-5) \sqrt{2+3 x-x^{2}} d x=\int \frac{(2 x-5)\left(2+3 x-x^{2}\right)}{\sqrt{2+3 x-x^{2}}} d x$ $=\left(\frac{2 x^{2}}{3}-3 x+\frac{1}{6}\right) R+\frac{17}{4} \arcsin \frac{3-2 x}{\sqrt{17}}$

197) $\int\left(x^{2}-3 x+5\right) \sqrt{3 x^{2}-2 x+6} d x$ $=\left(\frac{x^{3}}{4}-\frac{37 x^{2}}{36}+\frac{625}{216} x-\frac{503}{216}\right) R+\frac{2227}{216 \sqrt{3}} \lg (3 x-1+\sqrt{3} R)$ 198) $\int \frac{1}{\sqrt{a+b x^{2}}} d x= \pm \frac{1}{\sqrt{b}} \lg \left(b x \pm \sqrt{b} \sqrt{a+b x^{2}}\right)$ 199) $\int \frac{1}{\sqrt{a-b x^{2}}} d x=\frac{1}{\sqrt{b}} \arcsin x \sqrt{\frac{b}{a}}$ 
200) $\int \frac{d x}{\sqrt{2 b x+c x^{2}}}=\frac{1}{\sqrt{c}} \lg \left(b+c x+\sqrt{c} \sqrt{2 b x+c x^{2}}\right)$

201) $\int \frac{1}{\sqrt{2 b x-c x^{2}}} d x=-\frac{1}{\sqrt{c}} \arcsin \frac{b-c x}{b}$

202) $\int \sqrt{a+b x^{2}} d x=\frac{x}{2} \sqrt{a+b x^{2}}+\frac{a}{2 \sqrt{b}} \lg \left(b x+\sqrt{b} \sqrt{a+b x^{2}}\right)$

203) $\int \sqrt{a-b x^{2}} d x=\frac{x}{2} \sqrt{a-b x^{2}}+\frac{a}{2 \sqrt{b}} \arcsin x$

204) $\int \sqrt{2 a x+x^{2}} d x=\frac{a+x}{2} R-\frac{a^{2}}{2} \lg (a+x+R)$

205) $\int \sqrt{2 a x-x^{2}} d x=\frac{x-a}{2} R-\frac{a^{2}}{2} \arcsin \frac{a-x}{a}$

206) $\int x^{2} \sqrt{2 a x-x^{2}} d x=\left(\frac{x^{3}}{4}-\frac{a x^{2}}{12}-\frac{5 a^{2} x}{24}-\frac{5 a^{3}}{8}\right) R$

$-\frac{5 a^{4}}{8} \arcsin \frac{a-x}{a}$.

Die Relationen (10), S.145, fuhren zu den folgenden Formeln:

207) $\int \frac{x^{n}}{\sqrt{a+b x^{2}}} d x=\left(\alpha_{n-1} x^{n-1}+\alpha_{n-2} x^{n-2}\right.$

$$
\left.+\cdots+\alpha_{1} x+\alpha_{0}\right) \sqrt{a+b x^{2}}+K \int \frac{1}{\sqrt{a+b x^{2}}} d x .
$$

$a_{n-1}=\frac{1}{n b} ; \quad a_{n-2}=0 ; \quad \alpha_{n-3}=-\frac{n-1}{n-2} \cdot \frac{a}{b} \alpha_{n-1} ; \quad \alpha_{n-4}=0 ;$

$\alpha_{n-5}=-\frac{n-3}{n-4} \cdot \frac{a}{b} \alpha_{n-3} ; \alpha_{n-6}=0 ; \alpha_{n-7}=-\frac{n-5}{n-6} \cdot \frac{a}{b} \alpha_{n-5}$ usw.

$K=-a \alpha_{1}$, so dass für ungerade $n$ stets $K=0$ ist.

208) $\int \frac{x}{\sqrt{a+b x^{2}}} d x=\frac{1}{b} \int \frac{b x}{\sqrt{a+b x^{2}}} d x=\frac{1}{b} \sqrt{a+b x^{2}}$

209) $\int \frac{x^{2}}{\sqrt{a+b x^{2}}} d x=\frac{x}{2 b} \sqrt{a+b x^{2}}-\frac{a}{2 b \sqrt{b}} \lg (b x+\sqrt{b} R)$

210) $\int \frac{x^{3}}{\sqrt{a+b x^{2}}} d x=\left(\frac{x^{2}}{3 b}-\frac{2 a}{3 b^{2}}\right) \sqrt{a+b x^{2}}$

211) $\int \frac{x^{4}}{\sqrt{4+5 x^{2}}} d x=\left(\frac{x^{3}}{20}-\frac{3 x}{50}\right) R+\frac{6}{25 \sqrt{5}} \lg (5 x+\sqrt{5} R)$ 
212) $\int \frac{x^{5}}{\sqrt{3+2 x^{2}}} d x=\left(\frac{x^{4}}{10}-\frac{x^{2}}{5}+\frac{3}{5}\right) \sqrt{3+2 x^{2}}$.

Das Integral 207) kann auch mittelst einer Reduktionsfurmel ausgeführt werden.

$$
\begin{aligned}
& \int \frac{x^{n}}{\sqrt{a+b x^{2}}} d x=\frac{1}{b} \int x^{n-1} \frac{b x}{\sqrt{a+b x^{2}}} d x \\
& =\frac{1}{b} x^{n-1} \sqrt{a+b x^{2}}-\frac{n-1}{b} \int x^{n-2} \sqrt{a+b x^{2}} d x
\end{aligned}
$$

$\int x^{n-2} \sqrt{a+b x^{2}} d x=a \int \frac{x^{n-2}}{\sqrt{a+b x^{2}}} d x+b \int \frac{x^{n}}{\sqrt{a+b x^{2}}} d x$.

213) $\int \frac{x^{n}}{\sqrt{a+b x^{2}}} d x=\frac{1}{n b} x^{n-1} \sqrt{a+b x^{2}}-\frac{(n-1) a}{n b} \int \frac{x^{n-2}}{\sqrt{a+b x^{2}}} d x$

214) $\int \frac{x^{6}}{\sqrt{3-5 x^{2}}} d x=-\left(\frac{x^{5}}{30}+\frac{x^{3}}{40}+\frac{9 x}{400}\right) \sqrt{3-5 x^{2}}$

$$
+\frac{27}{400 \sqrt{5}} \arcsin \frac{5 x}{\sqrt{15}}
$$

Wenn $n$ eine gerade Zahl, so ist:

215) $\int \frac{x^{n}}{\sqrt{1-x^{2}}} d x=-\left(\frac{1}{n} x^{n-1}+\frac{1}{n} \cdot \frac{n-1}{n-2} x^{n-3}\right.$

$\left.+\frac{1}{n} \cdot \frac{n-1}{n-2} \cdot \frac{n-3}{n-4} x^{n-5}+\cdots+\frac{1}{n} \cdot \frac{n-1}{n-2} \cdots \frac{3}{2} x\right) \sqrt{1-x^{2}}$

$+\frac{1}{n} \cdot \frac{n-1}{n-2} \cdot \frac{n-3}{n-4} \ldots \frac{3}{2} \arcsin x$

216) $\int \frac{x^{6}}{\sqrt{1-x^{2}}} d x=-\left(\frac{x^{5}}{6}+\frac{5 x^{3}}{24}+\frac{5 x}{16}\right) \sqrt{1-x^{2}}+\frac{5}{16} \arcsin x$.

Wenn $n$ eine ungerade Zahl, so ist:

217) $\int \frac{x^{n}}{\sqrt{1-x^{2}}} d x=-\left(\frac{1}{n} x^{n-1}+\frac{1}{n} \cdot \frac{n-1}{n-2} x^{n-3}\right.$

$$
\left.+\frac{1}{n} \cdot \frac{n-1}{n-2} \cdot \frac{n-3}{n-4} x^{n-5}+\ldots\right) \sqrt{1-x^{2}}
$$

218) $\int \frac{x^{7}}{\sqrt{1-x^{2}}} d x=-\left(\frac{x^{6}}{7}+\frac{6 x^{4}}{35}+\frac{8 x^{2}}{35}+\frac{16}{35}\right) \sqrt{1-x^{2}}$. 


$$
S=\int \frac{1}{(x-\alpha) \sqrt{a+2 b x+c x^{2}}} d x
$$

Durch die Substitution $x-\alpha=\frac{1}{z}$ verwandelt $\operatorname{sich} S$ in folgendes Integral:

$$
S=-\int \frac{1}{\sqrt{c+2(b+c \alpha) z+\left(a+2 b \alpha+c \alpha^{2}\right) z^{2}}} d z .
$$

Je nachdem der Koeffizient von $z^{2}$ positiv, Null oder negativ wird, ist der Wert dieses Integrales verschieden.

1) $a+2 b \alpha+c \alpha^{2}>0$ und $=m$ gesetzt:

219) $\int \frac{1}{(x-\alpha) \sqrt{a+2 b x+c x^{2}}} d x$

$$
=\mp \frac{1}{\sqrt{m}} \lg \frac{a+b \alpha+(b+c \alpha) x \pm \sqrt{m} \sqrt{a+2 b} \overline{x+c x^{2}}}{x-\alpha} \text {. }
$$

2) $a+2 b \alpha+c \alpha^{2}<0$ und $=m$ :

220) $\int \frac{1}{(x-\alpha) \sqrt{a+2 b x+c x^{2}}} d x$

$$
=\frac{1}{\sqrt{-m}} \arcsin \frac{a+b \alpha+(b+c \alpha) x}{(x-\alpha) \sqrt{b^{2}-a c}} .
$$

Ist endlich 3) $a+2 b \alpha+c \alpha^{2}=0$,

so ist $\alpha$ eine Wurzel von $a+2 b x+c x^{2}=0$. Nennen wir die andere Wurzel $\beta$, so ist $c(x-\alpha)(x-\beta)=c x^{2}+2 b x+a$. Wird endlich $(x-\alpha)=z^{2}$ gesetzt, so ist $(x-\beta)=\left(z^{2}+\alpha-\beta\right)$ und

$$
S=\frac{2}{\sqrt{c}} \int \frac{1}{z^{2} \sqrt{z^{2}+\alpha-\beta}} d z .
$$

Durch die weitere Substitution $z=\frac{1}{u}$ geht $S$ tiber in:

$$
S=-\frac{2}{\sqrt{ } c} \int \frac{u}{\sqrt{(\alpha-\beta) u^{2}+1}} d u=-\frac{2}{\sqrt{c}} \frac{\sqrt{(\alpha-\beta) u^{2}+1}}{\alpha-\beta}
$$

und daraus durch die umgekehrten Substitutionen:

$$
S=-\frac{2}{c} \frac{\sqrt{c(x-\alpha)(x-\beta)}}{(\alpha-\beta)(x-\alpha)}=-\frac{2}{c} \frac{\sqrt{a+2 b x+c x^{2}}}{(\alpha-\beta)(x-\alpha)} .
$$


Da ferner $\alpha+\beta=-\frac{2 b}{c}$ ist, so kann $\alpha-\beta=2 \frac{b+c \alpha}{c}$ gesetzt werden. So wird schliesslich:

221) $\int \frac{1}{(x-\alpha) \sqrt{a+2 b x+c x^{2}}} d x=-\frac{\sqrt{a+2 b x+c x^{2}}}{(b+c \alpha)(x-\alpha)}$

222) $\int \frac{1}{(x-1) \sqrt{x^{2}+2 x+3}} d x=\frac{1}{\sqrt{6}} \lg \frac{2 x+4+\sqrt{6} R}{x-1}$

223) $\int \frac{1}{(x-2) \sqrt{x^{2}-6 x+1}} d x=-\frac{1}{\sqrt{7}} \arcsin \frac{x+5}{(x-2) \sqrt{8}}$

224) $\int \frac{1}{(x+1) \sqrt{1-x^{2}}} d x=-\sqrt{\frac{1-x}{1+x}}$.

Wenn $a>0$ ist, so ist:

225) $\int \frac{1}{x \sqrt{a+2 b x+c x^{2}}} d x=\frac{1}{\sqrt{a}} \lg \frac{a+b x+\sqrt{a} \sqrt{a+2 b x+c x^{2}}}{x}$.

Wenn $a<0$ ist, so ist:

226) $\int \frac{1}{x \sqrt{a+2 b x+c x^{2}}} d x=\frac{1}{\sqrt{-a}} \arcsin \frac{a+b x}{x \sqrt{b^{2}-a c}}$

227) $\int \frac{1}{x \sqrt{2 a x-x^{2}}} d x=-\frac{\sqrt{2 a x-x^{2}}}{a x}$

228) $\int \frac{1}{x \sqrt{1+x^{2}}} d x=\lg \frac{1-\sqrt{1+x^{2}}}{x}$

229) $\int \frac{1}{x \sqrt{x^{2}+x-1}} d x=\arcsin \frac{x-2}{x \sqrt{5}}$

230) $\int \frac{1}{x \sqrt{x^{2}-1}} d x=-\arcsin \frac{1}{x}$.

$$
S=\int \frac{1}{(x-\alpha)^{n} \sqrt{a+2 b x+c x^{2}}} d x .
$$

Durch die Substitution $x-\alpha=\frac{1}{z}$ erhält man:

$$
S=-\int \frac{z^{n-1}}{\sqrt{c+2(b+c \alpha) z+\left(a+2 b \alpha+c \alpha^{2}\right) z^{2}}} d z .
$$


231) $\int \frac{1}{(x-1)^{3} \sqrt{3-2 x^{2}}} d x=-\left(\frac{1}{2(x-1)^{2}}+\frac{3}{x-1}\right) \sqrt{3-2 x^{2}}$

$$
+7 \lg \frac{3-2 x-\sqrt{3-2 x^{2}}}{x-1}
$$

232) $\int \frac{1}{(x-2)^{4} \sqrt{1-4 x+x^{2}}} d x=\left(\frac{1}{9(x-2)^{3}}+\frac{2}{27(x-2)}\right)$ li

233) $\int \frac{1}{x^{2} \sqrt{a+2 b x+c x^{2}}} d x=-\frac{\sqrt{a+2 b x+c x^{2}}}{a x}$

$$
-\frac{b}{a} \int \frac{1}{x \sqrt{a+2 b x+c x^{2}}} d x
$$

234) $\int \frac{1}{x^{8} \sqrt{a+2 b x+c x^{2}}} d x=\left(\frac{-1}{2 a x^{2}}+\frac{3 b}{2 a^{2} x}\right) R$

$$
+\left(\frac{3 b^{2}}{2 a^{2}}-\frac{c}{2 a}\right) \int \frac{1}{x \sqrt{a+2 b x+c x^{2}}} d x
$$

235) $\int \frac{1}{x^{2} \sqrt{1-4 x+x^{2}}} d x=-\frac{1}{x} R+2 \lg \frac{1-2 x-R}{x}$

236) $\int \frac{1}{x^{3} \sqrt{1+x^{2}}} d x=-\frac{\sqrt{1+x^{2}}}{2 x^{2}}-\frac{1}{2} \lg \frac{1-\sqrt{1+x^{2}}}{x}$

237) $\int \frac{1}{x^{4} \sqrt{3-2 x+x^{2}}} d x=-\left(\frac{1}{9 x^{3}}+\frac{5}{54 x^{2}}+\frac{1}{54 x}\right) R$

$$
-\frac{2}{27 \sqrt{3}} \lg \frac{3-x-\sqrt{3} R}{x}
$$

238) $\int \frac{\sqrt{a+2 b x+c x^{2}}}{x} d x=\int \frac{b+c x}{\sqrt{a+2 b x+c x^{2}}} d x$

$$
\begin{gathered}
+a \int \frac{1}{x \sqrt{a+2 b x+c x^{2}}} d x+b \int \frac{1}{\sqrt{a+2 b x+c x^{2}}} d x . \\
\int \frac{\varphi(x)}{f(x)} \cdot \frac{1}{\sqrt{a+2 b x+c x^{2}}} d x .
\end{gathered}
$$

Man zerlegt $\frac{\varphi(x)}{f(x)}$ in Partialbrüche und damit das ganze Integral in eine Summe integrierbarer Teile. 
239) $\int \frac{3 x+1}{x^{2}-x-6} \cdot \frac{1}{\sqrt{3 x^{2}+4 x-7}} d x=\int\left(\frac{2}{x-3}+\frac{1}{x+2}\right) \frac{1}{R} d x$

$$
=\frac{1}{\sqrt{8}} \lg \frac{11 x-1-\sqrt{32} R}{x-3}-\frac{1}{\sqrt{3}} \arcsin \frac{4 x+11}{5(x+2)}
$$

240) $\int \frac{4 x+17}{x^{2}+x-6} \cdot \frac{1}{\sqrt{5-2 x^{2}}} d x=\frac{5}{\sqrt{3}} \arcsin \frac{5-4 x}{(x-2) \sqrt{10}}$

$$
-\frac{1}{\sqrt{13}} \arcsin \frac{6 x+5}{(x+3) \sqrt{10}}
$$

241) $\int \frac{3 x-5}{(x-1)^{2} \sqrt{x^{2}+4}} d x=\frac{2}{5} \cdot \frac{\sqrt{x^{2}+4}}{x-1}$

$$
+\frac{17}{5 \sqrt{5}} \lg \frac{x+4-\sqrt{5} \sqrt{x^{2}+4}}{x-1}
$$

242) $\int \frac{1}{\left(x^{2}-9\right) \sqrt{5+6 x-7 x^{2}}} d x=\frac{1}{6 \sqrt{40}} \arcsin \frac{7-9 x}{(x-3) \sqrt{11}}$ $-\frac{1}{6 \sqrt{76}} \arcsin \frac{12 x-2}{(x+3) \sqrt{11}}$

$$
S=\int \frac{1}{(x-p-q i) \sqrt{a+2 b x+c x^{2}}} d x .
$$

Dieses Integral geht hervor aus 219), wenn man $\alpha=p+q i$ setzt. Dann ist auch $m=a+2 b p+c\left(p^{2}-q^{2}\right)+2 q(b+c p) i$. Setzen wir $m=\varrho(\cos t+i \sin t)$, oder $\varrho \cos t=a+2 b p$ $+c\left(p^{2}-q^{2}\right), \varrho \sin t=2 q(b+c p)$, so sind $\varrho$ und $t$ hierdurch bestimmt. Zugleich ist:

$$
\frac{1}{\sqrt{m}}=\frac{1}{\sqrt{\varrho}} \cdot \frac{1}{\cos \frac{t}{2}+i \sin \frac{t}{2}}=\frac{1}{\sqrt{\varrho}}\left(\cos \frac{t}{2}-i \sin \frac{t}{2}\right) \text {. }
$$

$S=\frac{1}{\sqrt{\varrho}}\left(\cos \frac{t}{2}-i \sin \frac{t}{2}\right) \cdot \lg \left[a+b p+(b+c p) x-\sqrt{\varrho} \cos \frac{t}{2} R\right.$ $\left.+\left\{(b+c x) q-\sqrt{\varrho} \sin \frac{t}{2} R\right\} i\right]-\frac{1}{\sqrt{\varrho}}\left(\cos \frac{t}{2}-i \sin \frac{t}{2}\right) \lg (x-p-q i)$.

Wenn nun das vorstehende Integral bei der Zerlegung von $\frac{\varphi(x)}{f(x)}$ in Partialbriuche entstanden ist, so muss noch ein zweites 
existieren, welches sich von diesem nur durch das Vorzeichen von $i$ untersoheidet. In abgekürzter Bezeichnung mögen beide heissen:

$$
\begin{aligned}
& S=(A+B i) \lg (P+Q i)-(A+B i) \lg (x-p-q i) \\
& S_{1}=(A-B i) \lg (P-Q i)-(A-B i) \lg (x-p+q i)
\end{aligned}
$$

$S+S_{1}=A \lg \left(P^{2}+Q^{2}\right)+2 B \operatorname{arctg} \frac{P}{Q}-A \lg \left[(x-p)^{2}+q^{2}\right]$

Setzt $\operatorname{man} x^{2}=z$, so ist:

$$
+2 B \operatorname{arctg} \frac{x-p}{q} \text {. }
$$

243)

$$
\begin{aligned}
\int \frac{x}{\left(1+x^{2}\right) \sqrt{1-x^{4}}} d x & =\frac{1}{2} \int \frac{1}{(1+z) \sqrt{1-z^{2}}} d z \\
& =-\frac{1}{2} \sqrt{\frac{1-z}{1+z}}=-\frac{1}{2} \sqrt{\frac{1-x^{2}}{1+x^{2}}}
\end{aligned}
$$

244) $\int \sqrt{\frac{a+x}{a-x}} d x=\int \frac{a}{\sqrt{a^{2}-x^{2}}} d x+\int \frac{x}{\sqrt{a^{2}-x^{2}}} d x$

$$
=-\sqrt{a^{2}-x^{2}}+a \cdot \arcsin \frac{x}{a} \text {. }
$$

Setzt man $x^{8}=z^{2}$, so ist:

245) $\int \frac{\sqrt{x}}{\sqrt{a^{3}-x^{3}}} d x=\frac{2}{3} \int \frac{d z}{\sqrt{a^{3}-z^{2}}}=\frac{2}{3} \arcsin \sqrt{\frac{x^{3}}{a^{8}}}$.

Man setze $x^{2}=z$ in:

246) $\int x^{3} \sqrt{\frac{1+x^{2}}{1-x^{2}}} d x=\int \frac{x^{3}}{\sqrt{1-x^{4}}} d x+\int \frac{x^{5}}{\sqrt{1-x^{4}}} d x$

$$
\begin{aligned}
& =\frac{1}{2} \int \frac{z}{\sqrt{1-z^{2}}} d z+\frac{1}{2} \int \frac{z^{2}}{\sqrt{1-z^{2}}} d z \\
= & -\left(\frac{1}{2}+\frac{x^{2}}{4}\right) \sqrt{1-x^{4}}+\frac{1}{4} \arcsin x^{2}
\end{aligned}
$$

247) $\int\left(x+\sqrt{1+x^{2}}\right)^{n} d x=\frac{1}{2} \int\left(z^{n}+z^{n-2}\right) d z$ für $z=x+\sqrt{1+x^{2}}$

$$
=\frac{1}{2}\left[\frac{\left(x+\sqrt{1+x^{2}}\right)^{n+1}}{n+1}+\frac{\left(x+\sqrt{1+x^{2}}\right)^{n-1}}{n-1}\right] .
$$


§ 5. Exponential- und logarithmische Funktionen.

$$
\int f\left(e^{x}\right) d x
$$

Diese Funktionen werden vereinfacht durch $z=e^{x}$.

248) $\int e^{m x} d x=\frac{e^{m x}}{m}$

249) $\int\left(e^{3 x}+\sqrt{e^{x}}\right) d x=\frac{e^{3 x}}{3}+2 \sqrt{e^{x}}$

250) $\int \frac{e^{x}-1}{e^{x}+1} d x=\int \frac{z-1}{z(z+1)} d z=2 \lg \left(e^{x}+1\right)-x$

251) $\int \frac{1}{e^{x}+e^{-x}} d x=\operatorname{arctg} e^{x}$

252) $\int \frac{1}{\sqrt{a+b e^{x}}} d x=\frac{1}{\sqrt{a}} \lg \frac{\sqrt{a+b e^{x}}-\sqrt{a}}{\sqrt{a+b e^{x}}+\sqrt{a}} ; \quad z=\sqrt{a}+b e^{x}$

253) $\int \frac{1}{e^{x}} d x=-\frac{1}{e^{x}}$

254) $\int e^{x} \sqrt{1+e^{x}} d x=\frac{2}{3} \sqrt{\left(1+e^{x}\right)^{3}}$

255) $\int \frac{e^{x}}{\left(e^{x}-1\right)^{2}} d x=-\frac{1}{e^{x}-1}$

256) $\int\left(e^{x}+e^{-x}\right)^{2} d x=\frac{e^{2 x}-e^{-2 x}}{2}+2 x$

257) $\int \frac{e^{x}}{e^{x}+a} d x=\lg \left(e^{x}+a\right)$

258) $\int \frac{4 e^{x}+6 e^{-x}}{9 e^{x}-4 e^{-x}} d x=\frac{35}{36} \lg \left(e^{2 x}-\frac{4}{9}\right)-\frac{3 x}{2}$

259) $\int \frac{1}{e^{x}+e^{2 x}} d x=\lg \left(1+e^{x}\right)-\frac{1}{e^{x}}-x$

260) $\int \frac{e^{x}}{\left(e^{x}+a\right)^{n}} d x=-\frac{1}{(n-1)\left(e^{x}+a\right)^{n-1}}$.

$$
\int f\left(x, e^{x}\right) d x
$$

Neben der Substitution wird bei diesen Funktionen auch das Integrieren nach Teilen oft mit Vorteil angewendet. 
261) $\int e^{x} x^{4} d x=e^{x} x^{4}-4 \int e^{x} x^{3} d x$

262) $\int e^{x} x^{3} d x=e^{x} x^{3}-3 \int e^{x} x^{2} d x$

usw.

263) $\int e^{x} x^{4} d x=e^{x}\left(x^{4}-4 x^{3}+12 x^{2}-24 x+24\right)$

264) $\int e^{x} x^{n} d x=e^{x}\left(x^{n}-n x^{n-1}+n[n-1] x^{n-2}+\ldots\right)$

265) $\int e^{m x} x^{n} d x=\frac{e^{m x} x^{n}}{m}-\frac{n}{m} \int e^{m x} x^{n-1} d x$

266) $\int(a+b x)^{n} e^{m x} d x=\frac{e^{m x}(a+b x)^{n}}{m}-\frac{n b}{m} \int e^{m x}(a+b x)^{n-1} d x$.

Diese Reduktionsformel ist für positive und negative $m$ gleichmässig anwendbar. Man kann sie auch umkehren und $-p$ für $n-1$ setzen.

267) $\int(a+b x)^{-p} c^{m x} d x=-\frac{e^{m x}(a+b x)^{-p+1}}{(p-1)^{b}}$

$$
+\frac{m}{(p-1) b} \int e^{m x}(a+b x)^{-p+1} d x .
$$

Für $p=1$ ist diese Formel nicht mehr brauchbar, das Integral muss dann durch Reihenentwickelung weiter geführt werden.

268) $\int \frac{e^{x}}{x} d x=\int\left(\frac{1}{x}+1+\frac{x}{1.2}+\frac{x^{2}}{1.2 .3}+\ldots\right) d x$ $=\lg x+x+\frac{x^{2}}{4}+\frac{x^{8}}{18}+\cdots$

269) $\int x^{4} e^{2 x} d x=e^{2 x}\left(\frac{x^{4}}{2}-x^{8}+\frac{3 x^{2}}{2}-\frac{3 x}{2}+\frac{3}{4}\right)$

270) $\int x^{8} e^{-x} d x=-e^{-x}\left(x^{3}+3 x^{2}+6 x+6\right)$

271) $\int \frac{e^{x}}{x^{4}} d x=-e^{x}\left(\frac{1}{3 x^{3}}+\frac{1}{6 x^{2}}+\frac{1}{6 x}\right)+\frac{1}{6} \int \frac{e^{x}}{x} d x$

272) $\int \frac{e^{x}}{(x-a)^{n}} d x=e^{a} \int \frac{e^{z}}{z^{n}} d z$, wenn $z=x-a$. 
Exponentialfunktionen mit der Basis $a$ werden in sclche mit der Basis $e$ umgewandelt, indem man $a=e^{m}$ setzt.

273) $\int a^{b x} f(x) d x=\int e^{m b x} f(x) d x ; \quad m=\lg a$

274) $\int x^{8} a^{x} d x=a^{x}\left(\frac{x^{3}}{m}-\frac{3 x^{2}}{m^{2}}+\frac{6 x}{m^{3}}-\frac{6}{m^{4}}\right)$

275) $\int \frac{a^{x}}{x^{4}} d x=-a^{x}\left(\frac{1}{3 x^{3}}+\frac{m}{6 x^{2}}+\frac{m^{2}}{6 x}\right)+\frac{m^{3}}{6} \int \frac{a^{x}}{x} d x$

276) $\int f(x) d x=\int e^{z} f\left(e^{z}\right) d z$, wenn $z=\lg x$ ist.

277) $\int(\lg x)^{n} d x=\int e^{z} z^{n} d z=e^{z}\left(z^{n}-n z^{n-1}+\ldots\right)$

$$
=x\left[(\lg x)^{n}-n(\lg x)^{n-1}+n(n-1)(\lg x)^{n-2}-\ldots\right] .
$$

Dieses Integral lässt sich auch sehr leicht mittelst der Integration nach Teilen ausführen.

278) $\int(\lg x)^{n} d x=x(\lg x)^{n}-n \int(\lg x)^{n-1} d x$

279) $\int \lg x d x=x(\lg x-1)$

280) $\int(\lg x)^{2} d x=x\left([\lg x]^{2}-2 \lg x+2\right)$

281) $\int x^{n} \lg x d x=\frac{x^{n+1}}{n+1} \lg x-\frac{1}{n+1} \int x^{n} d x=\frac{x^{n+1}}{n+1}\left(\lg x-\frac{1}{n+1}\right)$

282) $\int x^{-n} \lg x d x=-\frac{1}{(n-1) x^{n-1}}\left(\lg x+\frac{1}{n-1}\right)$

283) $\int x^{3} \lg x d x=\frac{x^{4}}{4}\left(\lg x-\frac{1}{4}\right)$

284) $\int \frac{\lg x}{x^{4}} d x=-\frac{1}{3 x^{3}}\left(\lg x+\frac{1}{3}\right)$

285) $\int \frac{1}{x} \lg x d x=\frac{1}{2}(\lg x)^{2}$

286) $\int(a+b x)^{n} \lg x d x=\frac{(a+b x)^{n+1}}{(n+1) b} \lg x-\frac{1}{(n+1) b} \int \frac{(a+b x)^{n+1}}{x} d x$

287) $\int(4+3 x)^{2} \lg x d x=\frac{(4+3 x)^{3}}{9} \lg x-\frac{64}{9} \lg x-16 x-6 x^{2}-x^{3}$ 
288) $\int \lg (a+b x) d x=\frac{a+b x}{b}(\lg [a+b x]-1)$

289) $\int x^{n} \lg \left(a+b x^{m}\right) d x=\frac{x^{n+1}}{n+1} \lg \left(a+b x^{m}\right)-\frac{m b}{n+1} \int \frac{x^{m+n}}{a+b x^{m}} d x$

290) $\int \lg \left(a+b x^{2}\right) d x=x \lg \left(a+b x^{2}\right)-2 x+2 a \int \frac{1}{a+b x^{2}} d x$

291) $\int x \lg \left(1+x^{2}\right) d x=\frac{1+x^{2}}{2} \lg \left(1+x^{2}\right)-\frac{x^{2}}{2}$

292) $\int x^{3} \lg \left(x^{2}+3\right) d x=\frac{x^{4}}{4} \lg \left(x^{2}+3\right)-\frac{1}{4}\left(\frac{x^{4}}{2}-3 x^{2}+9 \lg \left(x^{2}+3\right)\right)$

293) $\int \frac{x}{\sqrt{a^{2}+x^{2}}} \lg x d x=\sqrt{a^{2}+x^{2}} \cdot \lg x-\int \frac{\sqrt{a^{2}+x^{2}}}{x} d x$

$$
=(\lg x-1) \sqrt{a^{2}+x^{2}}-a \lg \frac{a-\sqrt{a^{2}+x^{2}}}{x} .
$$

§6. Trigonometrische und eyklometrische Funktionen.

Die Grundformeln zur Integration dieser Funktionsarten werden durch Umkehrung der entsprechenden Differentialformeln gewonnen.

294) $\int \cos x d x=\sin x$

295) $\int \sin x d x=-\cos x$

296) $\int \cos n x d x=\frac{1}{n} \sin n x$

297) $\int \sin n x d x=-\frac{1}{n} \cos n x$

298) $\int \frac{1}{\sin ^{2} x} d x=-\operatorname{cotg} x$

299) $\int \frac{1}{\cos ^{2} x} d x=\operatorname{tg} x$

300) $\int \operatorname{tg} x d x=-\int \frac{-\sin x}{\cos x} d x=-\lg \cos x$

301) $\int \operatorname{cotg} x d x=\int \frac{\cos x}{\sin x} d x=\lg \sin x$ 
302) $\int \operatorname{tg} n x d x=-\frac{1}{n} \lg \cos n x$

303) $\int \operatorname{cotg} n x d x=\frac{1}{n} \lg \sin n x$

304) $\int \sin x \cos x d x=\frac{1}{2} \sin ^{2} x+C=-\frac{1}{2} \cos ^{2} x+C_{1}$

$$
=\frac{1}{2} \int \sin 2 x d x=-\frac{1}{4} \cos 2 x+C_{2} .
$$

Je 2 dieser 3 Werte differieren nur um eine Konstante

305) $\int \sin ^{2} x d x=\frac{1}{2} \int(1-\cos 2 x) d x=\frac{1}{2} x-\frac{1}{4} \sin 2 x$

306) $\int \cos ^{2} x d x=\int\left(1-\sin ^{2} x\right) d x=\frac{1}{2} x+\frac{1}{4} \sin 2 x$.

Aus 300) und 301) folgt:

307) $\int(\operatorname{tg} x+\operatorname{cotg} x) d x=\lg \sin x-\lg \cos x=\lg \operatorname{tg} x$, oder

308) $\int \frac{2}{\sin 2 x} d x=\lg \operatorname{tg} x$, oder:

309) $\int \frac{1}{\sin x} d x=\lg \operatorname{tg} \frac{x}{2}$

310) $\begin{aligned} & \int \frac{1}{\cos x} d x=\int \frac{1}{\sin \left(\frac{\pi}{2}-x\right)} d x=-\int \frac{1}{\sin z} d z=-\lg \operatorname{tg} \frac{z}{2} \\ & =-\lg \operatorname{tg}\left(\frac{\pi}{4}-\frac{x}{2}\right)=\lg \operatorname{cotg}\left(\frac{\pi}{4}-\frac{x}{2}\right)=\lg \operatorname{tg}\left(\frac{\pi}{4}+\frac{x}{2}\right)\end{aligned}$

Höhere Potenzen von $\sin x$ und $\cos x$ können mit Hilfe von Reduktionsformeln integriert werden. Sei:

$$
S_{n}=\int \sin ^{n} x d x
$$

$\int \sin ^{n} x d x=\int \sin ^{n-1} x \sin x d x$

$$
=-\cos x \sin ^{n-1} x+(n-1) \int \sin ^{n-2} x \cos ^{2} x d x
$$

$S_{n}=-\cos x \sin ^{n-1} x+(n-1) \int \sin ^{n-2} x\left(1-\sin ^{2} x\right) d x$

$S_{n}=-\cos x \sin ^{n-1} x+(n-1) S_{n-2}-(n-1) S_{n}$

$n S_{n}=-\cos x \sin ^{n-1} x+(n-1) S_{n-2}$

311) $\int \sin ^{n} x d x=-\frac{1}{n} \cos x \sin ^{n-1} x+\frac{n-1}{n} \int \sin ^{n-2} x d x$. 
Wird die Formel umgekehrt und $n-2$ durch $-p$ ersetzt, so entsteht:

312) $\begin{aligned} \int(\sin x)^{-p} d x=-\frac{1}{p-1} \cos x & (\sin x)^{-p+1} \\ & +\frac{p-2}{p-1} \int(\sin x)^{-p+2} d x\end{aligned}$

Die beiden Formeln dienen dazu, den Exponenten von $\sin x$ auf 2 oder auf $1 \mathrm{zu}$ erniedrigen und so das Integral auf eine nach früheren Formeln ausführbare Form zu bringen.

313) $\int \sin ^{3} x d x=-\frac{1}{3}\left(\sin ^{2} x+2\right) \cos x$

314) $\int \sin ^{4} x d x=-\left(\frac{1}{4} \sin ^{8} x+\frac{3}{8} \sin x\right) \cos x+\frac{3 x}{8}$

315) $\int \sin ^{5} x d x=-\left(\frac{1}{5} \sin ^{4} x+\frac{4}{15} \sin ^{2} x+\frac{8}{15}\right) \cos x$

316) $\int \frac{1}{\sin ^{3} x} d x=-\frac{\cos x}{2 \sin ^{2} x}+\frac{1}{2} \lg \operatorname{tg} \frac{x}{2}$

317) $\int \frac{1}{\sin ^{4} x} d x=-\left(\frac{1}{3 \sin ^{3} x}+\frac{2}{3 \sin x}\right) \cos x$.

Die Reduktionsformeln für $\cos ^{n} x$ werden auf dieselbe Weise direkt gewonnen wie diejenigen für $\sin ^{n} x$; sie können aber auch aus 311) und 312) dadurch abgeleitet werden, dass man $\cos x=\sin \left(\frac{\pi}{2}-x\right)=\sin z$ setzt.

318) $\int \cos ^{n} x d x=\int \sin ^{n}\left(\frac{\pi}{2}-x\right) d x=-\int \sin ^{n} z d z$.

Wird das Integral entwickelt und darin wieder $\sin z$ durch $\cos x$ und $\cos z$ durch $\sin x$ ersetzt, so erhält man:

319) $\int \cos ^{n} x d x=\frac{1}{n} \cos ^{n-1} x \sin x+\frac{n-1}{n} \int \cos ^{n-2} x d x$

320) $\int(\cos x)^{-p} d x=\frac{1}{p-1} \sin x(\cos x)^{-p+1}+\frac{p-2}{p-1} \int(\cos x)^{-p+2} d x$

321) $\int \cos ^{4} x d x=\left(\frac{1}{4} \cos ^{3} x+\frac{3}{8} \cos x\right) \sin x+\frac{3}{8} x$

322) $\int \cos ^{5} x d x=\left(\frac{1}{5} \cos ^{4} x+\frac{4}{15} \cos ^{2} x+\frac{8}{15}\right) \sin x$ 
323) $\int \frac{1}{\cos ^{3} x} d x=\frac{\sin x}{2 \cos ^{2} x}+\frac{1}{2} \lg \operatorname{tg}\left(\frac{\pi}{4}+\frac{x}{2}\right)$

324) $\int \frac{1}{\cos ^{5} x} d x=\frac{\sin x}{4 \cos ^{4} x}+\frac{3 \sin x}{8 \cos ^{2} x}+\frac{3}{8} \lg \operatorname{tg}\left(\frac{\pi}{4}+\frac{x}{2}\right)$.

Mit Hilfe der beiden Formeln:

$$
\sin n x=\frac{e^{n x i}-e^{-n x i}}{2 i}, \cos n x=\frac{e^{n x i}+e^{-n x i}}{2}
$$

lassen $\operatorname{sich} \sin ^{n} x$ und $\cos ^{n} x$ in Funktionen der Vielfachen des Bogens umwandeln; wir wollen an einem Beispiel zeigen, wie dies geschieht.

$$
\begin{aligned}
\sin ^{5} x= & \left(\frac{e^{i x}-e^{-i x}}{2 i}\right)^{5}=\frac{e^{5 i x}-5 e^{3 i x}+10 e^{i x}-10 e^{-i x}+5 e^{-3 i x}-e^{-5 i x}}{32 i} \\
& =\frac{1}{16}\left[\frac{e^{5 i x}-e^{-5 i x}}{2 i}-5 \frac{e^{3 i x}-e^{-3 i x}}{2 i}+10 \frac{e^{i x}-e^{-i x}}{2 i}\right] \\
& =\frac{1}{16}[\sin 5 x-5 \sin 3 x+10 \sin x] .
\end{aligned}
$$

325) $\int \sin ^{5} x d x=\frac{1}{16}\left[-\frac{\cos 5 x}{5}+\frac{5 \cos 3 x}{3}-10 \cos x\right]$

326) $\int \cos ^{6} x d x=\frac{\sin 6 x}{192}+\frac{3 \sin 4 x}{64}+\frac{15 \sin 2 x}{64}+\frac{5 x}{16}$

327) $\int \sin ^{6} x d x=-\frac{1}{32}\left[\frac{\sin 6 x}{6}-\frac{3 \sin 4 x}{2}+\frac{15 \sin 2 x}{2}\right]+\frac{5 x}{16}$

328) $\int \cos ^{7} x d x=\frac{1}{64}\left[\frac{\sin 7 x}{7}+\frac{7 \sin 5 x}{5}+7 \sin 3 x+35 \sin x\right]$

329) $\int \cos ^{8} x d x=\frac{1}{128}\left[\frac{\sin 8 x}{8}+\frac{4 \sin 6 x}{3}+7 \sin 4 x\right.$

$+28 \sin 2 x]+\frac{35 x}{128}$

Wird $\sin x=y$ gesetzt, bez. $\cos x=y$, so erhält man leicht:

$$
\int \sin ^{n} x d x=\int \frac{y^{n}}{\sqrt{1-y^{2}}} d y ; \int \cos ^{n} x d x=-\int \frac{y^{n}}{\sqrt{1-y^{2}}} d y \text {. }
$$

330) $\int \sin ^{3} x d x=\int\left(1-\cos ^{2} x\right) \sin x d x=-\int\left(1-y^{2}\right) d y$

$$
=-\left(y-\frac{y^{8}}{3}\right)=\frac{\cos ^{3} x}{3}-\cos x
$$


331) $\int \cos ^{5} x d x=\int\left(1-\sin ^{2} x\right)^{2} \cos x d x=\int\left(1-y^{2}\right)^{2} d y$ $=\frac{\sin ^{5} x}{5}-\frac{2 \sin ^{8} x}{3}+\sin x$

332) $\int \frac{d x}{\sin x}=\int \frac{\sin x d x}{1-\cos ^{2} x}=\int-\frac{d y}{1-y^{2}}=\frac{1}{2} \lg \frac{1-y}{1+y}$

$$
=\frac{1}{2} \lg \frac{\sin ^{2} \frac{x}{2}}{\cos ^{2} \frac{x}{2}}=\lg \operatorname{tg} \frac{x}{2}
$$

333) $\int \frac{d x}{\sin ^{6} x}=-\left[\frac{1}{5 \sin ^{5} x}+\frac{4}{15 \sin ^{3} x}+\frac{8}{15 \sin x}\right] \cos x$

334) $\int \frac{d x}{\sin ^{7} x}=-\left[\frac{1}{6 \sin ^{6} x}+\frac{5}{24 \sin ^{4} x}+\frac{5}{16 \sin ^{2} x}\right] \cos x+\frac{5}{16} \lg \operatorname{tg} \frac{x}{2}$ 335) $\int \frac{d x}{\cos ^{6} x}=\frac{\sin x}{5 \cos ^{5} x}+\frac{4 \sin x}{15 \cos ^{3} x}+\frac{8}{15} \operatorname{tg} x$.

Setzt $\operatorname{man} \sin x=\frac{1}{y}$ oder $\cos x=\frac{1}{y}$, so wird man finden:

$$
\int \frac{d x}{\sin ^{n} x}=-\int \frac{y^{n-1}}{\sqrt{y^{2}-1}} d y ; \int \frac{d x}{\cos ^{n} x}=\int \frac{y^{n-1}}{\sqrt{y^{2}-1}} d y .
$$

336) $\int \frac{d x}{\cos ^{2} x}=\left[\frac{1}{6 \cos ^{6} x}+\frac{5}{24 \cos ^{4} x}+\frac{5}{16 \cos ^{2} x}\right] \sin x+\frac{5}{16} \lg \operatorname{tg}\left(\frac{\pi}{4}+\frac{x}{2}\right)$ 337) $\int \frac{d x}{\cos ^{8} x}=\left[\frac{1}{7 \cos ^{7} x}+\frac{6}{35 \cos ^{5} x}+\frac{8}{35 \cos ^{3} x}+\frac{16}{35 \cos x}\right] \sin x$ 338) $\int \sin ^{n} x \cos x d x=\frac{\sin ^{n+1} x}{n+1}$

339) $\int \cos ^{n} x \sin x d x=-\frac{\cos ^{n+1} x}{n+1}$.

$$
\int \sin ^{m} x \cos ^{n} x d x .
$$

Gewöhnlich wird dieses Integral durch Integration nach Teilen auf ein einfacheres Integral derselben Art reduziert: $\int \sin ^{m} x \cos ^{n} x d x=-\frac{\sin ^{m-1} x \cos ^{n+1} x}{n+1}+\frac{m-1}{n+1} \int \sin ^{m-2} x \cos ^{n+2} x d x$. $\sin ^{m-2} x \cos ^{n+2} x=\sin ^{m-2} x \cos ^{n} x\left(1-\sin ^{2} x\right)=\sin ^{m-2} x \cos ^{n} x$ $-\sin ^{m} x \cos ^{n} x$. 
Wird hiernach das letzte Integral zerlegt und dann reduziert, so erhält man:

340) $\int \sin ^{m} x \cos ^{n} x d x=-\frac{\sin ^{m-1} x \cos ^{n+1} x}{m+n}$

Setzt $\operatorname{man} m=p+2$, so wird:

$$
+\frac{m-1}{m+n} \int \sin ^{m-2} x \cos ^{n} x d x .
$$

$\int \sin ^{p+2} x \cos ^{n} x d x=-\frac{\sin ^{p+1} x \cos ^{n+1} x}{p+n+2}+\frac{p+1}{p+n+2} \int \sin ^{p} x \cos ^{n} x d x$.

341) $\int \sin ^{p} x \cos ^{n} x d x=\frac{\sin ^{p+1} x \cos ^{n+1} x}{p+1}$

$$
+\frac{p+n+2}{p+1} \int \sin ^{p+2} x \cos ^{n} x d x .
$$

Während 340) dazu dient, ein positives $m$ zu verkleinern, wird durch 341) ein negatives $p$ vergrössert; in beiden Fällen bleibt $n$ ungeändert. Wird wieder $\left(\frac{\pi}{2}-x\right)$ statt $x,-d x$ statt $d x$ gesetzt, so entstehen aus den beiden letzten Formeln die folgenden:

342) $\int \cos ^{m} x \sin ^{n} x d x=\frac{\cos ^{m-1} x \sin ^{n+1} x}{m+n}$

$$
+\frac{m-1}{n+m} \int \cos ^{m-2} x \sin ^{n} x d x
$$

343) $\int \sin ^{n} x \cos ^{n} x d x=-\frac{\sin ^{n+1} x \cos ^{p+1} x}{p+1}$

$$
+\frac{p+n+2}{p+1} \int \sin ^{n} x \cos ^{p+2} x d x
$$

344) $\int \sin ^{4} x \cos ^{2} x d x=\left[\frac{\sin ^{5} x}{6}-\frac{\sin ^{3} x}{24}-\frac{\sin x}{16}\right] \cos x+\frac{x}{16}$

345) $\int \sin ^{5} x \cos ^{2} x d x=\left[\sin ^{6} x-\frac{\sin ^{4} x}{5}-\frac{4 \sin ^{2} x}{15}-\frac{8}{15}\right] \frac{\cos x}{7}$.

Aufgaben dieser Art können auch so behandelt werden:

346) $\int \sin ^{2} x \cos ^{2} x d x=\int \sin ^{2} x\left(1-\sin ^{2} x\right) d x$

$$
=\left[\frac{\sin ^{3} x}{4}-\frac{\sin x}{8}\right] \cos x+\frac{x}{8}
$$


347) $\int \sin ^{3} x \cos ^{3} x d x=\int \sin ^{3} x\left(1-\sin ^{2} x\right) \cos x d x=\frac{\sin ^{4} x}{4}-\frac{\sin ^{6} x}{6}$

348) $\int \sin ^{3} x \cos ^{6} x d x=\frac{\cos ^{9} x}{9}-\frac{\cos ^{7} x}{7}$

349) $\begin{aligned} \int \sin ^{4} x \cos ^{5} x d x=\int \sin ^{4} x(1 & \left.-\sin ^{2} x\right)^{2} \cos x d x \\ & =\frac{\sin ^{5} x}{5}-\frac{2 \sin ^{7} x}{7}+\frac{\sin ^{9} x}{9} .\end{aligned}$

$$
\int \frac{\sin ^{n} x}{\cos x} d x, \quad \int \frac{\cos ^{n} x}{\sin x} d x .
$$

Wird im ersten Integral $\sin x=y$, im zweiten $\cos x=y$ gesetzt, so wandeln sich dieselben um in:

$$
-\int \frac{y^{n}}{y^{2}-1} d y \text { und } \int \frac{y^{n}}{y^{2}-1} d y \text {. }
$$

Die Division wird ausgeftihrt, der Quotient integriert, in dem Restbruch aber vor der Integration die umgekehrte Substitution vollzogen, weil so ein leicht ausführbares Integral entsteht.

350) $\int \frac{\sin ^{8} x}{\cos x} d x=-\frac{\sin ^{2} x}{2}-\lg \cos x$

351) $\int \frac{\sin ^{6} x}{\cos x} d x=-\frac{\sin ^{5} x}{5}-\frac{\sin ^{3} x}{3}-\sin x+\lg \operatorname{tg}\left(\frac{\pi}{4}+\frac{x}{2}\right)$

352) $\int \frac{\cos ^{4} x}{\sin x} d x=\frac{\cos ^{8} x}{3}+\cos x+\lg \operatorname{tg} \frac{x}{2}$

353) $\int \frac{\cos ^{5} x}{\sin x} d x=\frac{\cos ^{4} x}{4}+\frac{\cos ^{2} x}{2}+\lg \sin x$

354) $\int \frac{\sin x}{\cos ^{n} x} d x=\frac{1}{(n-1) \cos ^{n-1} x}$

355) $\int \frac{\cos x}{\sin ^{n} x} d x=-\frac{1}{(n-1) \sin ^{n-1} x}$.

Statt mittelst der Formeln 341) und 343) negative Exponenten zu reduzieren, kőnnen derartige Aufgaben auch so behandelt werden:

356) $\int \frac{\sin ^{5} x}{\cos ^{4} x} d x=\int \frac{\sin x\left(1-\cos ^{2} x\right)^{2} d x}{\cos ^{4} x}=\frac{1}{3 \cos ^{3} x}-\frac{2}{\cos x}-\cos x$ 
357) $\int \frac{\cos ^{3} x}{\sin ^{2} x} d x=-\frac{1+\sin ^{2} x}{\sin x}$

358) $\int \frac{\cos ^{6} x}{\sin ^{5} x} d x=\left[\cos ^{4} x-\frac{25 \cos ^{2} x}{8}+\frac{15}{8}\right] \frac{\cos x}{\sin ^{4} x}+\frac{15}{8} \lg \operatorname{tg} \frac{x}{2}$

359) $\int \frac{\sin ^{4} x}{\cos ^{3} x} d x=\left[-\sin ^{3} x+\frac{3 \sin x}{2}\right] \frac{1}{\cos ^{2} x}-\frac{3}{2} \lg \operatorname{tg}\left[\frac{\pi}{4}+\frac{x}{2}\right]$

360) $\int \frac{\sin ^{7} x}{\cos ^{4} x} d x=\left[-\frac{\sin ^{6} x}{3}-2 \sin ^{4} x+8 \sin ^{2} x-\frac{16}{3}\right] \frac{1}{\cos ^{3} x}$

361) $\int \frac{d x}{\sin x \cos x}=\lg \operatorname{tg} x$

362) $\int \frac{d x}{\sin ^{2} x \cos ^{2} x}=-2 \operatorname{cotg} 2 x$

363) $\int \frac{d x}{\sin ^{8} x \cos x}=-\frac{1}{2 \sin ^{2} x}+\lg \operatorname{tg} x$

364) $\int \frac{d x}{\sin ^{2} x \cos ^{4} x}=\frac{1}{3 \sin x \cos ^{3} x}-\frac{8}{3} \operatorname{cotg} 2 x$

Durch teilweise Integration erhält man:

365) $\int \operatorname{tg}^{n} x d x=\int \sin ^{n} x \cos ^{-n} x d x=\frac{\operatorname{tg}^{n-1} x}{n-1}-\int \operatorname{tg}^{n-2} x d x$

366) $\int \operatorname{cotg}^{n} x d x=-\frac{\operatorname{eotg}^{n-1} x}{n-1}-\int \operatorname{cotg}^{n-2} x d x$

367) $\int \operatorname{tg}^{5} x d x=\frac{1}{4} \operatorname{tg}^{4} x-\frac{1}{2} \operatorname{tg}^{2} x-\lg \cos x$

368) $\int \operatorname{tg}^{8} x d x=\frac{1}{7} \operatorname{tg}^{7} x-\frac{1}{5} \operatorname{tg}^{5} x+\frac{1}{3} \operatorname{tg}^{3} x-\operatorname{tg} x+x$

369) $\int \operatorname{cotg}^{6} x d x=-\frac{\operatorname{cotg}^{5} x}{5}+\frac{\operatorname{cotg}^{3} x}{3}-\operatorname{cotg} x-x$

370) $\int \operatorname{cotg}^{7} x d x=-\frac{\operatorname{cotg}^{6} x}{6}+\frac{\operatorname{cotg}^{4} x}{4}-\frac{\operatorname{cotg}^{2} x}{2}-\lg \sin x$.

$\int e^{a x} \sin b x d x=-\frac{e^{a x} \cos b x}{b}+\frac{a}{b} \int e^{a x} \cos b x d x$

$\int e^{a x} \cos b x d x=\frac{e^{a x} \sin b x}{b}-\frac{a}{b} \int e^{a x} \sin b x d x$. 
Wird je eines der beiden Integrale eliminiert, so erhält man:

371) $\int e^{a x} \sin b x d x=\frac{e^{a x}}{a^{2}+b^{2}}[a \sin b x-b \cos b x]$

372) $\int e^{a x} \cos b x d x=\frac{e^{a x}}{a^{2}+b^{2}}[a \cos b x+b \sin b x]$.

Setzt man in beiden Formeln $-x$ statt $x$, so gehen daraus die folgenden hervor:

373) $\int e^{-a x} \sin b x d x=-\frac{e^{-a x}}{a^{2}+b^{2}}[a \sin b x+b \cos b x]$

374) $\int e^{-a x} \cos b x d x=-\frac{e^{-a x}}{a^{2}+b^{2}}[a \cos b x-b \sin b x]$

375) $\int e^{a x} \sin m x \cos n x d x=\frac{1}{2} \int e^{a x} \sin (m+n) x d x$

$$
+\frac{1}{2} \int e^{a x} \sin (m-n) x d x
$$

376) $\int e^{a x} \sin ^{4} b x d x=\frac{1}{8} \int e^{a x}(\cos 4 b x-4 \cos 2 b x+3) d x$

377) $\int e^{a x} \cos ^{3} b x d x=\frac{1}{4} \int e^{a x}(\cos 3 b x+3 \cos b x) d x$

378) $\int x^{n} \sin x d x=-x^{n} \cos x+n \int x^{n-1} \cos x d x$

379) $\int x^{n} \cos x d x=x^{n} \sin x-n \int x^{n-1} \sin x d x$

380) $\int \frac{\sin x}{x^{n}} d x=-\frac{\sin x}{(n-1) x^{n-1}}+\frac{1}{n-1} \int \frac{\cos x}{x^{n-1}} d x$

381) $\int \frac{\cos x}{x^{n}} d x=-\frac{\cos x}{(n-1) x^{n-1}}-\frac{1}{n-1} \int \frac{\sin x}{x^{n-1}} d x$.

Wird $\cos x=\frac{1-y^{2}}{1+y^{2}}$ gesetzt, so ist $y=\operatorname{tg} \frac{x}{2}$ und

382) $\int \frac{d x}{a+\cos x}=\frac{2}{a-1} \int \frac{d y}{y^{2}+\frac{a+1}{a-1}}$

$$
n \quad=\frac{2}{\sqrt{a^{2}-1}} \operatorname{arctg} y \sqrt{\frac{a-1}{a+1}} \text {, wenn } \frac{a+1}{a-1}>0,
$$




$$
\int \frac{d x}{a+\cos x}=\frac{-1}{\sqrt{1-a^{2}}} \lg \frac{y-\sqrt{\frac{1+a}{1-a}}}{y+\sqrt{\frac{1+a}{1-a}}}, \text { wenn } \frac{a+1}{a-1}<0 .
$$

Für $a=1$ ist

383) $\int \frac{d x}{1+\cos x}=\int \frac{d x}{2 \cos ^{2} \frac{x}{2}}=\operatorname{tg} \frac{x}{2}$.

Ebenso findet man für $\sin x=\frac{y^{2}-1}{y^{2}+1}$, dass $y=\operatorname{tg}\left(\frac{\pi}{4}+\frac{x}{2}\right)$ und 384) $\int \frac{d x}{a+\sin x}=\frac{2}{a+1} \int \frac{d y}{y^{2}+\frac{a-1}{a+1}}$

$$
\begin{aligned}
& "=\frac{2}{\sqrt{a^{2}-1}} \operatorname{arctg} y \sqrt{\frac{a+1}{a-1}}, \text { wenn } \frac{a-1}{a+1}>0, \\
& "=\frac{+1}{\sqrt{1-a^{2}}} \lg \frac{y-\sqrt{\frac{1-a}{1+a}}}{y+\sqrt{\frac{1-a}{1+a}}}, \text { wenn } \frac{a-1}{a+1}<0 .
\end{aligned}
$$

Für $a=1$ wird

385) $\int \frac{d x}{1+\sin x}=\int \frac{d x}{2 \sin ^{2}\left(\frac{\pi}{4}+\frac{x}{2}\right)}=-\operatorname{cotg}\left(\frac{\pi}{4}+\frac{x}{2}\right)$

386) $\int \frac{\cos x}{a+\cos x} d x=\int\left(1-\frac{a}{a+\cos x}\right) d x=x-a \int \frac{d x}{a+\cos x}$ 387) $\int \frac{\sin x}{a+\sin x} d x=x-a \int \frac{d x}{a+\sin x}$

388) $\int \frac{d x}{a+b \sin x+c \cos x}=S$

$S=m \int \frac{d x}{m a+m b \sin x+m c \cos x}=m \int \frac{d x}{m a+\sin \lambda \sin x+\cos \lambda \cos x}$, wenn $m b=\sin \lambda, m c=\cos \lambda$, also $m=\frac{1}{\sqrt{b^{2}+c^{2}}}$ gesetzt wird. Weiter ist $\lambda=\operatorname{arctg} \frac{b}{c}$ und 


$$
S=m \int \frac{d x}{a m+\cos (x-\lambda)} .
$$

Hiermit ist das gegebene Integral auf 382) zurückgefuhrt.

389) $\int \frac{d x}{\sin x+\cos x}=\frac{1}{\sqrt{2}} \lg \operatorname{tg}\left(\frac{x}{2}+\frac{\pi}{8}\right) ; \quad m=\frac{1}{\sqrt{2}} ; \quad \lambda=\frac{\pi}{4}$.

Ist $m a-1=0$, oder $a=\sqrt{b^{2}+c^{2}}$, so ist:

390) $\int \frac{d x}{a+b \sin x+c \cos x}=\frac{1}{a} \cdot \frac{c \sin x-b \cos x}{a+b \sin x+c \cos x}$.

\section{Bestimmte Integrale.}

In die Integrale, wie sie bisher betrachtet wurden, ging stets eine willkürliche, unbestimmte Konstante ein; deswegen nennt man sie unbestimmte Integrale. Ist für die Konstante einmal ein Wert gewählt worden, so darf im Verlaufe der Rechnung nicht ohne weiteres eine Änderung dieses Wertes vorgenommen werden: dann ist das Integral nur eine Funktion der Veränderlichen $x$. In dem Ausdrucke

$$
\int f(x) d x=F(x)+C
$$

ist es ja nun ohne Bedenken, dem $x$ auf der rechten Seite einen festen Wert, etwa $b$, beizulegen und $F(b)+C$ zu schreiben. Das geht aber links nicht an, da das Symbol $d x$ nicht in $d b$ übergehen kann, weil für eine konstante Grösse $b$ der Zuwachs $\Delta b$ stets Null ist. Man müsste also anders schreiben, etwa

$$
\left|\int f(x) d x\right|_{x=b} \text { oder bequemer } \int^{b} f(x) d x .
$$

Man hat daher

$$
\int^{b} f(x) d x=F(b)+C
$$


Ebenso ist für eine andere Konstante $a$

$$
\int f(x) d x=F(a)+C
$$

und also innerhalb derselben Rechnung

$$
\int^{b} f(x) d x-\int{ }^{a} f(x) d x=F(b)-F(a) .
$$

Die linke Seite kann man bequemer schreiben

$\int_{a}^{b} f(x) d x=F(b)-F(a)=[F(b)+C]-[F(a)+C]$.

Dieser Ausdruck heisst: Das bestimmte Integral. Er ist von der Wahl der unbestimmten Integrationskonstanten unabhängig geworden und daher stets gleich der Differenz aus den Werten des unbestimmten Integrals fur die obere und für die untere Grenze unter Annahme der gleichen Integrationskonstante für beide.

Aus (11) folgen die Formeln:

$$
\begin{gathered}
\int_{a}^{b} f(x) d x+\int_{b}^{c} f(x) d x=[F(b)-F(a)]+[F(c)-F(b)] \\
=F(c)-F(a)=\int_{a}^{c} f(x) d x \\
\int_{a}^{c} f(x) d x-\int_{a}^{b} f(x) d x=\int_{b}^{c} f(x) d x \\
\int_{a}^{c} f(x) d x-\int_{b}^{c} f(x) d x=\int_{a}^{b} f(x) d x \\
\int_{a}^{b} f(x) d x=-\int_{b}^{a} f(x) d x
\end{gathered}
$$

Nehmen wir als obere Grenze eine Veränderliche $u$, so wird

$$
\int_{a}^{u} f(x) d x=F(u)-F(a)
$$

eine Funktion allein von $u$, nicht mehr von $x$. Nach der Definition des Integrals gilt die Formel

$$
\frac{d}{d u} \int_{a}^{u} f(x) d x=\frac{d}{d u} F(u)=f(u) .
$$

Der Differentialquotient des bestimmten Integrals nach seiner oberen Grenze ist gleich dem Integranden 
Aus (13) folgt, wenn wir die untere Grenze veränderlich machen

$$
\frac{d}{d v} \int_{v}^{b} f(x) d x=-f(v)
$$

Schreibt man (14) in der Form

$$
d \int_{a}^{u} f(x) d x=f(u) d u
$$

so erkennt man, dass der Zuwachs des bestimmten Integrals bei veränderlicher oberer Grenze gleichzeitig mit dem $\mathrm{Zu}$ wachse dieser Grenze unendlich klein wird, falls $f(u)$ endlich bleibt.

Es ist folglich das bestimmte Integral eine stetige Funktion seiner oberen Grenze. Dabei kann der Integrand unstetig werden, d. h. es darf (vgl. S. 76, Z. 16 ff.) $f(x+0)$ von $f(x-0)$ versehieden sein. Geschieht dies z. B. an der zwischen $a$ und $b$ gelegenen Stelle $c$, so müssen wir jetzt definieren

$$
\begin{aligned}
\int_{a}^{b} f(x) d x=\int_{a}^{c-0} f(x) d x+\int_{c+0}^{b} f(x) d x \\
=F(b)-F(c+0)+F(c-0)-F(a),
\end{aligned}
$$

Bedenkt man die Bedeutung eines Differentials, so kann man $\left(14^{b}\right)$ in der Form

$$
\int_{a}^{u+\Delta u} f(x) d x-\int_{a}^{u} f(x) d x=\int_{u}^{u+\Delta u} f(x) d x=f(u) \Delta u
$$

schreiben, wenn man unter $\Delta u$ einen beliebig kleinen Zuwachs versteht. Dadureh erhält man der Reihe nach

$$
\begin{aligned}
& \int_{a}^{a+\Delta u} f(x) d x=\Delta u f(a), \\
& \int_{a+\Delta u}^{a+2 \Delta u} f(x) d x=\Delta u f(a+\Delta u), \\
& \int_{a+2 \Delta u}^{a+3 \Delta u} f(x) d x=\Delta u f(a+2 \Delta u),
\end{aligned}
$$


und durch Summierung von $n$ solehen Gleichungen

$\int_{a+n \Delta u}^{a+n}$

$$
\begin{aligned}
f(x) d x=\Delta u[f(a)+f(a+\Delta u) & +f(a+2 \Delta u) \\
& +\ldots+f(a+(n-1) \Delta u)] .
\end{aligned}
$$

Wenn man hierin $n \Delta u=b-a$ setzt und dabei zugleich $n$ ins Unendliche gehen und also $\Delta u$ zu Null werden lässt, dann entsteht

$$
\begin{aligned}
\int_{a}^{b} f(x) d x=\lim _{\delta=0} \delta \cdot[f(a)+f(a+\delta) & +f(a+2 \delta) \\
& +\ldots+f(b-\delta)] .
\end{aligned}
$$

Diese Formel giebt eine Definition des bestimmten Integrals ohne Vermittelung des unbestimmten und ermöglicht dadurch auch eine direkte Berechnung. Wir wollen ein Beispiel dafür geben :

$$
\begin{aligned}
\int_{a}^{b} e^{x} d x & =\lim _{\delta=0} \delta \cdot\left[e^{a}+e^{a+\delta}+\ldots+e^{a+(n-1) \delta}\right] \\
& =\lim \delta e^{a}\left(1+e^{\delta}+e^{2 \delta}+\ldots+e^{(n-1) \delta}\right) \\
& =\lim \delta \cdot e^{a} \frac{e^{n \delta}-1}{e^{\delta}-1} \\
& =\lim \frac{\delta}{e^{\delta}-1}\left(e^{b}-e^{a}\right)=e^{b}-e^{a}
\end{aligned}
$$

da die Grenze des Quotienten sich nach den Regeln der Differentialrechnung als 1 ergiebt.

Es kann vorkommen, dass der Integrand $f(x)$ für einen Wert $c$ zwischen $a$ und $b$ unendlich gross wird, und dass man trotzdem dem Integral einen Sinn geben kann. Wir setzen nämlich als Definition in diesem Falle

$$
\int_{a}^{b} f(x) d x=\lim _{\delta=0} \int_{a}^{c-\delta} f(x) d x+\lim _{\bullet=0} \int_{c+\ell}^{b} f(x) d x,
$$

unter der Voraussetzung, dass jedes der beiden Integrale rechts einen bestimmten endlichen, von $\delta$ und bezw. von $\varepsilon$ unabhängigen Wert auch für den Grenzlubergang besitze. So ist $\mathrm{z}$. B. 


$$
\begin{aligned}
& \int_{0}^{9} \frac{d x}{\sqrt[3]{(x-1)^{2}}}=\lim _{\delta=0} \int_{0}^{1-\delta} \frac{d x}{\sqrt[3]{(x-1)^{2}}}+\lim _{\bullet=0} \int_{1+e \sqrt[3]{(x-1)^{2}}}^{9} \frac{d x}{\sqrt[3]{(x-1)}} \\
& =\lim _{\delta=0}\left[3(x-1)^{\frac{1}{3}}\right]_{x=0}^{x=1-\delta}+\lim _{\varepsilon=0}\left[3(x-1)^{\frac{1}{3}}\right]_{x=1+\bullet}^{x=9} \\
& =-3-\lim _{\delta=0} 3 \delta^{\frac{1}{3}}-\lim _{\varepsilon=0} 3 \varepsilon^{\frac{1}{3}}+6=3 \text {, }
\end{aligned}
$$

während

$$
\int_{0}^{9} \frac{d x}{\sqrt[3]{(x-1)^{4}}}=\lim _{\delta=0} \int_{0}^{1-\delta} \frac{d x}{\sqrt[3]{(x-1)^{4}}}+\lim _{\bullet=0} \int_{1+\cdot \sqrt[3]{(x-1)^{4}}}^{9}
$$

keinen Sinn hat.

Eine ähnliche Erweiterung des Integralbegriffes ist auch in dem Falle möglich, dass eine oder dass beide Grenzen ins Unendliche gehen. Hier setzt man als Definition

$$
\int_{a}^{\infty} f(x) d x=\lim _{\delta=0} \int_{a}^{\frac{1}{\delta}} f(x) d x \quad(\delta \text { positiv })
$$

unter der Voraussetzung, dass das Integral rechts einen bestimmten endlichen, von $\delta$ unabhängigen Wert auch für den Grenzubergang besitze. So ist z. B.

$\int_{0}^{\infty} e^{-x} d x=\lim _{\delta=0} \int_{0}^{\frac{1}{\delta}} e^{-x} d x=\lim _{\delta=0}\left[-e^{-x}\right]_{0}^{\frac{1}{\delta}}=1-\lim _{\delta=0} e^{-\frac{1}{\delta}}=1 ;$ während $\int_{0}^{\infty} e^{x} d x$ keinen Sinn hat. Ähnlich ist aufzufassen

$$
\int_{-\infty}^{\infty} f(x) d x=\lim _{\delta=0} \int_{0}^{\frac{1}{\delta}} f(x) d x+\lim _{e=0} \int_{-\frac{1}{\delta}}^{0} f(x) d x .
$$

Aufgaben.

391) $\int_{a}^{b} m x^{n} d x=\frac{m}{n+1}\left(b^{n+1}-a^{n+1}\right)$

392) $\int_{-2}^{+2} x^{4} d x=\frac{64}{5}$

393) $\int_{a}^{b}(a+b x) d x=\frac{2 a b+b^{3}-2 a^{2}-a^{2} b}{2}$ 
394) $\int_{0}^{3}\left(2 x+3 x^{2}\right) d x=36$
395) $\int_{-\frac{1}{2}}^{0}\left(1+\frac{3 x}{2}-x^{2}\right) d x=\frac{13}{48}$

396) $\int_{0}^{3 a} \frac{x^{2}-3 a x}{x-4 a} d x=a^{2}\left(\frac{15}{2}-8 \lg 2\right)$

397) $\int_{3}^{5} \frac{x}{x^{2}-4} d x=\frac{1}{2} \lg \frac{21}{5}$

398) $\int_{0}^{a} \frac{d x}{x^{2}+a^{2}}=\frac{\pi}{4 a}$

399) $\int_{0}^{\infty} \frac{d x}{x^{2}+1}=\frac{\pi}{2}$

400) $\int_{2}^{7} \frac{x}{x^{2}+2 x-3} d x=\frac{1}{4} \lg 6+\frac{3}{4} \lg 2$

401) $\int_{0}^{a} d x \sqrt{x}=\frac{2}{3} \sqrt{a^{3}}$

402) $\int_{2}^{7} d x \sqrt{x+2}=\frac{38}{3}$

403) $\int_{0}^{a} d x \sqrt{a^{2}-x^{2}}=\frac{a^{2} \pi}{4}$

404) $\int_{0}^{a} 3 x \sqrt{x^{2}+4 a^{2}} d x=a^{3}(5 \sqrt{5}-8)$

405) $\int_{0}^{a} \frac{d x}{\sqrt{a^{2}-x^{2}}}=\frac{\pi}{2}$

406) $\int_{0}^{1} \frac{x}{\sqrt{1-x^{2}}} d x=1$

407) $\int_{1}^{4} \frac{x}{\sqrt{2+4 x}} d x=\frac{3}{2} \sqrt{2}$

408) $\int_{0}^{a} \frac{x^{2}}{\sqrt{a x-x^{2}}} d x=\frac{3 a^{2} \pi}{8}$

409) $\int_{0}^{2 b} d x \sqrt{2 b x-x^{2}}=\frac{b^{2} \pi}{2}$

410) $\int_{0}^{1} \frac{x^{2 n}}{\sqrt{1-x^{2}}} d x=\frac{1.3 .5 \ldots(2 n-1)}{2.4 .6 \ldots \ldots \ldots .2 n} \cdot \frac{\pi}{2}$ 


$$
\begin{aligned}
& \text { 411) } \int_{0}^{1} \frac{x^{2 n+1}}{\sqrt{1-x^{2}}} d x=\frac{2.4 .6 \ldots \ldots \ldots 2 n}{3.5 .7 \ldots(2 n+1)} \\
& \text { 412) } \int_{0}^{1} e^{x} d x=e-1 \\
& \text { 413) } \int_{0}^{1} x e^{x} d x=1 \\
& \text { 414) } \int_{0}^{2} e^{a x} d x=\frac{e^{2 a}-1}{a} \\
& \text { 415) } \int_{0}^{\infty} e^{-a x} d x=\frac{1}{a} \\
& \text { 416) } \int_{-1}^{+1} a^{x} d x=\frac{a^{2}-1}{a \lg a} \\
& \text { 417) } \int_{1}^{2} \frac{d x}{x}=\lg 2 \\
& \text { 418) } \int_{1}^{e} x^{2} \lg x d x=\frac{1+2 e^{3}}{9} \\
& \text { 419) } \int_{0}^{\frac{\pi}{4}} \sin x d x=1-\sqrt{0,5} \\
& \text { 420) } \int_{0}^{\pi} 2 a \sin \frac{x}{2} d x=4 a
\end{aligned}
$$

421) $\int_{0}^{\frac{\pi}{3}}(2 \cos x-1) d x=0,6848 \ldots=\sqrt{3} \ldots \frac{\pi}{3}$

422) $\int_{0}^{\pi} \sin m x d x=\frac{1-\cos m \pi}{m}$

423) $\int_{0}^{\pi} \cos m x d x=\frac{\sin m \pi}{m}$

424) $\int_{0}^{\pi} \sin 2 a x d x=0$, wenn $a$ ganzzahlig ist.

425) $\int_{0}^{\frac{\pi}{2}} \sin ^{2} 2 x d x=\frac{\pi}{4}$

426) $\int_{0}^{\frac{\pi}{2}} \sin ^{2 n} x d x=\int_{0}^{\frac{\pi}{2}} \cos ^{2 n} x d x=\frac{1.3 \ldots(2 n-1)}{2.4 \ldots \ldots \ldots \ldots 2 n} \cdot \frac{\pi}{2}$ 
427) $\int_{0}^{\frac{\pi}{2}} \sin ^{2 n+1} x d x=\int_{0}^{\frac{\pi}{2}} \cos ^{2 n+1} x d x=\frac{2.4 \ldots \ldots \ldots 2 n}{3.5 \ldots(2 n+1)}$

428) $\int_{0}^{\frac{\pi}{2}}\left(\cos ^{4} x-\cos ^{6} x\right) d x=\frac{\pi}{32}$

429) $\int_{\frac{\pi}{4}}^{\frac{\pi}{2}} \sin ^{3} x d x=\frac{5}{6} \sqrt{\frac{1}{2}}$

430) $\int_{0}^{1}\left(1-\sqrt[3]{x^{2}}\right)^{\frac{3}{2}} d x=\frac{3 \pi}{32}$.

Man setzt $x=\sin ^{3} \varphi$, und $\varphi=0$ für $x=0, \varphi=\frac{\pi}{2}$ für $x=1$ als Grenze.

431) $\int_{0}^{\frac{\pi}{2}} e^{2 x} \sin ^{2} x d x=\frac{3 e^{\pi}-1}{8}$

432) $\int_{-\frac{\pi}{2}}^{+\frac{\pi}{2}} \cos ^{3} x d x=\frac{4}{3}$

433) $\int_{0}^{\frac{\pi}{4}} \operatorname{tg} x d x=\frac{1}{2} \lg 2$

434) $\int_{0}^{\frac{\pi}{2}} \frac{d x}{1+\cos x}=1$. 


\section{Anwendung der Differential- und Integral- rechnung auf Geometrie.}

\& 1. Tangente und Normale ebener Kurven.

Wird an die Kurve $y=f(x)$ dureh den Punkt $(x, y)$ eine Tangente gelegt, so ist deren Steigungsmass $=\frac{d y}{d x}$ (S. 8), und ihre Gleichung:

$$
Y-y=\frac{d y}{d x}(X-x),
$$

wenn $X, Y$ die variabelen Koordinaten eines auf der Tangente beweglichen Punktes und $x, y$ die Koordinaten des Berührungspunktes bedeuten. Erscheint die Kurvengleichung in der Form: $f(x, y)=0$, so muss für $\frac{d y}{d x}$ der Wert $-\frac{\partial f}{\partial x}: \frac{\partial f}{\partial y}$ substituiert werden; man erhält dann als Gleichung der Tangente:

$$
(Y-y) \frac{\partial f}{\partial y}+(X-x) \frac{\partial f}{\partial x}=0 .
$$

Diese Form der Gleichung der Tangente kann sehr leicht aus der homogenen Form der Kurvengleichung abgeleitet werden. Bezeichnen wir durch $f(x, y, z)=0$ eine homogene Funktion vom $n^{\text {ten }}$ Grad, so ist nach Euler:

$$
x \frac{\partial f}{\partial x}+y \frac{\partial f}{\partial y}+z \frac{\partial f}{\partial z}=n f(x, y, z)=0 .
$$



Wird in (2) fuir $-\left(x \frac{\partial f}{\partial x}+y \frac{\partial f}{\partial y}\right)$ der Wert $z \frac{\partial f}{\partial z}$ sub-
stituiert, so entsteht:

$$
\frac{\partial f}{\partial x} X+\frac{\partial f}{\partial y} Y+\frac{\partial f}{\partial z} z=0 .
$$

Um (2) aus der gegebenen Kurvengleichung zu gewinnen, macht man sie also homogen, bestimmt die D.-Q. $\frac{\partial f}{\partial x}, \frac{\partial f}{\partial y}, \frac{\partial f}{\partial z}$ und setzt dieselben in (4) ein. Wird dann $z=1$ gesetzt, so geht (4) über in (2).

Beispiel: $\frac{x^{2}}{a^{2}}+\frac{y^{2}}{b^{2}}-1=0$ ist die gewöhnliche, $\frac{x^{2}}{a^{2}}+\frac{y^{2}}{b^{2}}-z^{2}=0$ die homogene Form der Ellipsengleichung, $\frac{\partial f}{\partial x}=\frac{2 x}{a^{2}}, \frac{\partial f}{\partial y}=\frac{2 y}{b^{2}}$, $\frac{\partial f}{\partial z}=-2 z$. Homogene Form der Tangentengleichung: $\frac{x X}{a^{2}}+\frac{y Y}{b^{2}}-z^{2}=0 . \quad z=1$ giebt $\frac{x X}{a^{2}}+\frac{y Y}{b^{2}}-1=0$ als gewöhnliche Form der Gleichung der Tangente.

Wird der Lauf einer Kurve durch die beiden Gleichungen bestimmt: $x=\varphi(t), \quad y=\psi(t), \quad$ so ist $\frac{d y}{d x}=\frac{d y}{d t}: \frac{d x}{d t}$, und die Gleichung der Tangente:

$$
(Y-y) \frac{d x}{d t}-(X-x) \frac{d y}{d t}=0 .
$$

Ist endlich $r=f(t)$ die Gleichung einer Kurve auf Polarkoordinaten bezogen, so ist: $x=r \cos t ; y=r \sin t$; $\frac{d x}{d t}=\frac{d r}{d t} \cos t-r \sin t ; \frac{d y}{d t}=\frac{d r}{d t} \sin t+r \cos t$.

Die Normale. Unter der Normale verstehen wir die gerade Linie, welche senkrecht zur Tangente durch deren Berïhrungspunkt gelegt ist. Ihr Steigungsmass ist daher $=-\frac{1}{\frac{d y}{d x}}$. Den verschiedenen Formen der Kurvengleichung entsprechen die nachfolgenden Formen der Normalengleichung 


$$
\begin{array}{ll}
y=f(x) & (Y-y) \frac{d y}{d x}+(X-x)=0 \\
f(x, y)=0 & (Y-y) \frac{\partial f}{\partial x}-(X-x) \frac{\partial f}{\partial y}=0 \\
x=\varphi(t), y=\psi(t) & (Y-y) \frac{d y}{d t}+(X-x) \frac{d x}{d t}=0 .
\end{array}
$$

Für die nachfolgenden Kurven soll die Gleichung der Tangente aufgestellt werden:

1) Hyperbel: $\frac{x^{2}}{a^{2}}-\frac{y^{2}}{b^{2}}-1=0 ; \mathrm{Tg}: \frac{x X}{a^{2}}-\frac{y Y}{b^{2}}-1=0$.

2) Parabel: $\quad y^{2}-2 p x=0$; Tg: $y Y-p(X+x)=0$.

3) Neilsche Parabel: $x^{3}-p y^{2}=0$;

Tg: $3 x^{2} X-2 p y Y-p y^{2}=0$.

4) Cissoide: $y^{2}(2 r-x)-x^{3}=0$;

Tg: $2 y(x-2 r) Y+\left(3 x^{2}+y^{2}\right) X-2 r y^{2}=0$.

5) Descartessches Blatt: $x^{3}-3 a x y+y^{3}=0$;

Tg: $\left(x^{2}-a y\right) X+\left(y^{2}-a x\right) Y-a x y=0$.

6) Die Lemniskate erhält man in folgender Weise: Zwei Punkte $A$ und $B$ sind durch ihre Entfernung $A B=2 e$ Fig. 12.

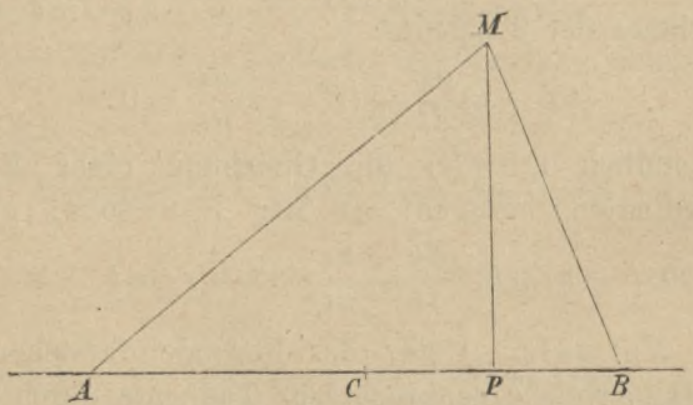

gegeben; man soll den geometrischen Ort aller Punkte $M$ finden, die so liegen, dass das Produkt der beiden Leitstrahlen $M A$ und $M B$ konstant ist. Für $M A=r$, $M B=r_{1}$ muss $r \times r_{1}=a^{2}$ sein. $C A=C B=e ; C P=x$, $M P=y ; \quad\left[y^{2}+(e+x)^{2}\right]\left[y^{2}+(e-x)^{2}\right]=a^{4} ; \quad\left(y^{2}+x^{2}\right)^{2}$ 
$=2 e^{2}\left(x^{2}-y^{2}\right)+a^{4}-e^{4}$. Dem besonderen Fall $a=e$ entspricht die Gleichung:

$$
\left(x^{2}+y^{2}\right)^{2}-2 a^{2}\left(x^{2}-y^{2}\right)=0 .
$$

Durch die Substitution: $x=r \cos t, y=r \sin t$ erbält man als Polargleichung: $r=a \sqrt{2 \cos 2 t}=b \sqrt{\cos 2 t}$

Wird in einem Kreis senkrecht zu einem Durchmesser eine Sehne $=2 a$ gezogen, so zerlegt dieselbe den Durchmesser in 2 Teile, deren Produkt $=a^{2}$ ist, und die deshalb als Leitstrahlen zur Konstruktion eines Kurvenpunktes benutzt werden können.

Als Gleichung der Tangente findet man:

$$
\left(x^{2}+y^{2}-a^{2}\right) x X+\left(x^{2}+y^{2}+a^{2}\right) y Y+a^{2}\left(y^{2}-x^{2}\right)=0
$$

7) Logarithmische Linie: $y=a^{x}$;

Tangente: $Y-y=(X-x) a^{x} \lg a$.

8) Kettenlinie: $y=\frac{m}{2}\left(e^{\frac{x}{m}}+e^{-\frac{x}{m}}\right)$;

Tangente: $Y-y=\frac{1}{2}\left(e^{\frac{x}{m}}-e^{-\frac{x}{m}}\right)(X-x)$.

Da $\operatorname{tg} \alpha=\frac{d y}{d x}=\frac{1}{2}\left(e^{\frac{x}{m}}-e^{-\frac{x}{m}}\right)=\frac{\sqrt{y^{2}-m^{2}}}{m}$ ist, so findet man den Richtungswinkel $a$ einer Tangente, indem man aus der Ordinate des Berührungspunktes als Hypotenuse und $m$ als Kathete ein rechtwinkeliges Dreieck konstruiert.

9) Die Cykloide. Wenn ein Kreis (Fig. 13) eine Gerade $A B$ in $A$ bertihrt und dann auf der Geraden hinrollt, so beschreibt der anfängliche Bertihrungspunkt $A$ eine Cykloide. Jeder vollständigen Umdrehung entspricht ein Kurvenzweig. Es sei $A P=x, M P=y, M C=a$. Der Winkel zwischen $C M$ und dem Radius $C B$ zum neuen Berührungspunkte sei $t$, so ist $\operatorname{arc} M B=a t$. Da $A B=\operatorname{arc} M B$, so ist $x=A P=A B-M D=a(t-\sin t) ; y=M P=C B$ $-C D=a(1-\cos t)$. Beide Gleichungen bestimmen zu- 
sammen den Lauf der Cykloide. Wird $t$ eliminiert, so erhält man: $x=a$ arc $\cos \frac{a-y}{a}-\sqrt{2 a y-y^{2}}$.

Fig. 13.

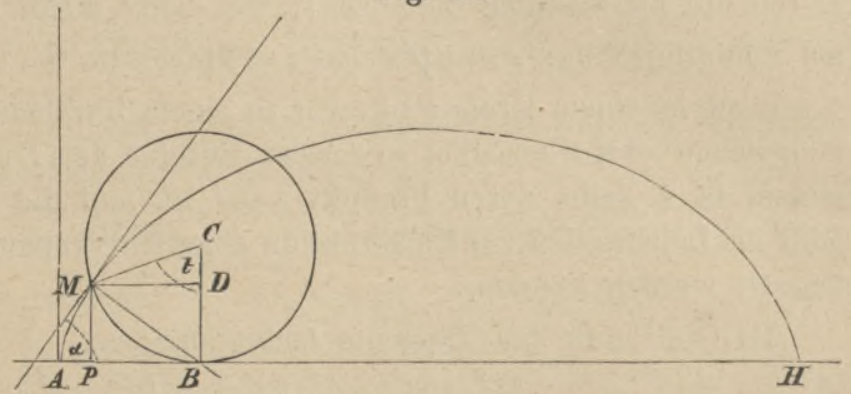

Gleichung der T'angente: $Y-y=(X-x) \operatorname{cotg} \frac{t}{2}$

$$
\text { n } \quad \text { N Normale: } \quad Y-y=-(X-x) \operatorname{tg} \frac{t}{2} \text {. }
$$

Um zu beweisen, dass die Normale in $M$ durch den Berührungspunkt $B$ des Erzeugungskreises geht, zeigen wir, dass $X=a t, Y=0$, die Koordinaten von $B$, in Verbindung mit $x=a(t-\sin t) \quad y=a(1-\cos t)$, den Koordinaten von $M$, der Gleichung der Normale genügen. 10) Die Epicykloide. Rollt der Kreis, dessen Mittelpunkt $C$ ist (Fig. 14), mit seiner konvexen Seite auf der konvexen Fig. 14.

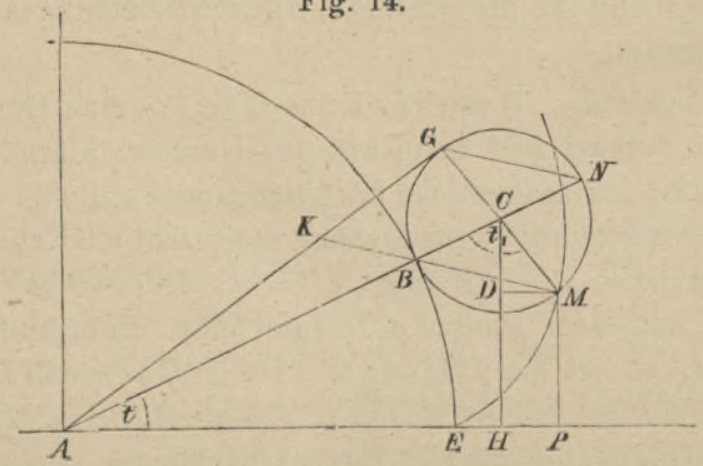


Seite des festen Kreises mit dem Mittelpunkt $A$ hin, so beschreibt Punkt $M$ bei einer Umwälzung des rollenden Kreises den ersten Zweig einer Epicykloide. Der rollende Kreis und der Bahnkreis berühren sich von aussen.

Es sei $A B=r, \quad C B=a, \quad A P=x, \quad M P=y$. Die Winkel $E A B$ und $M C B$ werden mit $t$ und $t_{1}$ bezeichnet; folglich hat man are $B E=r t$, are $B M=a t_{1}$, $\operatorname{arc} B E=\operatorname{arc} B M$, oder $r t=a t_{1}$, d. h. $t_{1}=\frac{r t}{a} ; x=A H$ $+D M ; \quad A H=(a+r) \cos t ; \quad D M=a \sin \left(t+t_{1}-\frac{\pi}{2}\right)$ $=-a \cos \left(t+t_{1}\right)=-a \cos \left(\frac{a+r}{a}\right) t ; y=C H-C D ;$ $C H=(a+r) \sin t ; C D=a \cos \left(t+t_{1}-\frac{\pi}{2}\right)=a \sin \left(t+t_{1}\right)$ $=a \sin \left(\frac{a+r}{a}\right) t$. So erhält man als Gleichungen der Epicykloide:

$$
\begin{aligned}
& x=(a+r) \cos t-a \cos \left(\frac{a+r}{a}\right) t \\
& y=(a+r) \sin t-a \sin \left(\frac{a+r}{a}\right) t .
\end{aligned}
$$

Gleichung der Tangente: $Y-y=(X-x) \operatorname{tg}\left(\begin{array}{c}\frac{r+2 a}{2 a} \\ \text { " } \quad \text { Normale: } Y-y=-(X-x) \operatorname{cotg}\left(\frac{r+2 a}{2 a}\right) t .\end{array}\right.$

Soll wieder bewiesen werden, dass die Normale in $M$ durch den Beriihrungspunkt $B$ des Erzeugungskreises geht, so muss man zeigen, dass $X=r \cos t, Y=r \sin t$, die Koordinaten von $B$, in Verbindung mit den Werten für $x$ und $y$ die Gleichung der Normale befriedigen.

11) Die Cardioide ist ein besonderer Fall der Epicykloide; er entspricht der Bedingung $a=r$. Thre Gleichungen sind daher: $x=2 a \cos t-a \cos 2 t=a\left(2 \cos t+1-2 \cos ^{2} t\right)$; $y=2 a \sin t-a \sin 2 t=2 a \sin t(1-\cos t)$. Wird das System in der Weise transformiert, dass man $a-x$ statt $x$ 
setzt, so erhält man: $x=2 a \cos t(\cos t-1) ; \frac{y}{x}=-\operatorname{tg} t$; $\cos t=\frac{-x}{\sqrt{x^{2}+y^{2}}} ; x^{2}+y^{2}=4 a^{2}(1-\cos t)^{2} ; \quad\left(x^{2}+y^{2}\right)^{2}$ $-4 a x\left(x^{2}+y^{2}\right)-4 a^{2} y^{2}=0$. Setzt man: $x=r \cos \varphi$, $y=r \sin \varphi$, so gewinnt man die Polargleichung:

$$
r=2 a(1+\cos \varphi)=4 a \cos ^{2} \frac{\varphi}{2} .
$$

12) Lässt man in den Gleichungen der Epicycloide - $a$ an die Stelle von $+a$ treten, so erhält man die Нypocykloide, wenn $r>a$, dagegen die Perioykloide, wenn $r<a$ ist. Bei der Entstehung der Hypocykloide wird der rollende Kreis von dem berührenden Bahnkreise umschlossen. Bei der Entstehung der Pericykloide umschliesst der rollende Kreis den bertihrenden Bahnkreis.

13) Die archimedische Spirale: $r=a t$, oder $x=a t \cos t$, $y=a t \sin t$.

$$
\text { Tg.: }(Y-y)(\cos t-t \sin t)-(X-x)(\sin t+t \cos t)=0 .
$$

14) Die logarithmische Spirale: $r=a^{t}$.

Tg.: $\frac{Y-y}{X-x}=\frac{\lg a \sin t+\cos t}{\lg a \cos t-\sin t}=k$ (=Steigungsmass d.Tg.)

Das Steigungsmass $k_{1}$ des Leitstrahls ist gleich $\operatorname{tg} t$. Man kann beweisen, dass $\operatorname{tg} v=\frac{k-k_{1}}{1+k k_{1}}=\frac{1}{\lg a}$, d. h. dass der Winkel $v$, welchen die Tangente mit dem Leitstrahl bildet, bei dieser Kurve konstant ist.

8 2. Doppelpunkte, Rüekkehrpunkte (Spitzen), konjugierte (isolierte) Punkte.

Wenn in Formel (2) durch die Koordinaten des Berührungspunktes $\frac{\partial f}{\partial x}=0$ und $\frac{\partial f}{\partial y}=0$ wird, so nimmt $\frac{d y}{d x}$ die unbestimmte Form $\frac{0}{0}$ an, und damit erscheint für diesen Punkt, (den wir als im Endlichen gelegen annehmen), auch die Richtung der 
Tangente in unbestimmter Form. Als wahren Wert dieses unbestimmten Ausdrucks finden wir:

$$
\frac{d y}{d x}=-\frac{\frac{d\left(\frac{\partial f}{\partial x}\right)}{d x}}{\frac{d\left(\frac{\partial f}{\partial y}\right)}{d x}}=-\frac{\frac{\partial^{2} f}{\partial x^{2}}+\frac{\partial^{2} f}{\partial x \partial y} \cdot \frac{d y}{d x}}{\frac{\partial^{2} f}{\partial y \partial x}+\frac{\partial^{2} f}{\partial y^{2}} \cdot \frac{d y}{d x}}
$$

und daraus folgt:

$$
\frac{d y}{d x}=\frac{-\frac{\partial^{2} f}{\partial x \partial y} \pm \sqrt{\left(\frac{\partial^{2} f}{\partial x \partial y}\right)^{2}-\frac{\partial^{2} f}{\partial x^{2}} \cdot \frac{\partial^{2} f}{\partial y^{2}}}}{\frac{\partial^{2} f}{\partial y^{2}}}
$$

Vorausgesetzt, dass dieser Ausdruck nicht gleichfalls unbestimmt wird, giebt er uns für $\frac{d y}{d x}$ im allgemeinen zwei verschiedene Werte, und daraus folgern wir, dass die Kurve in diesem Punkt zwei verschiedene Tangenten besitzt, was nur dadurch möglich wird, dass sich 2 Kurvenäste in diesem Punkt durchschneiden. Ein solcher Punkt wird Doppelpunkt genannt. Damit aber die beiden Werte reell und verschieden ausfallen, muss $\left(\frac{\partial^{2} f}{\partial x \partial y}\right)^{2}>\frac{\partial^{2} f}{\partial x^{2}} \cdot \frac{\partial^{2} f}{\partial y^{2}}$ sein. Ist dagegen $\left(\frac{\partial^{2} f}{\partial x \partial y}\right)^{2}=\frac{\partial^{2} f}{\partial x^{2}} \cdot \frac{\partial^{2} f}{\partial y^{2}}$, so fallen die beiden Tangenten in Eine zusammen, die Kurvenschleife verschwindet alsdann, und der Doppelpunkt wird zu einem Rückkehrpunkt. Wird endlich $\left(\frac{\partial^{2} f}{\partial x \partial y}\right)^{2}<\frac{\partial^{2} f}{\partial x^{2}} \cdot \frac{\partial^{2} f}{\partial y^{2}}$, so wird $\frac{d y}{d x}$ imaginär. Der Punkt gehört dann der Kurve an, lässt aber keine Tangente zu, weil er mit den übrigen Teilen der Kurve nicht in Verbindung steht. Wir nennen ihn einen isolierten oder konjugierten Punkt. Doppelpunkte, Rückkehrpunkte und isolierte Punkte haben hiernach die gemeinsame Eigenschaft, dass ihre Koordinaten den Gleichungen: 


$$
f(x, y)=0, \quad \frac{\partial f}{\partial x}=0, \quad \frac{\partial f}{\partial y}=0
$$

genügen müssen. Aber man hat:

Einen Doppelpunkt, wenn $\left(\frac{\partial^{2} f}{\partial x \partial y}\right)^{2}>\frac{\partial^{2} f}{\partial x^{2}} \cdot \frac{\partial^{2} f}{\partial y^{2}}$

" Rückkehrpunkt, " isolierten Punkt, "

Wird aus (9) der Wert für $\frac{d y}{d x}$ in (1) substituiert, so entsteht die für die Variabelen $X$ und $Y$ quadratische Gleichung: $(Y-y)^{2} \frac{\partial^{2} f}{\partial y^{2}}+2(Y-y)(X-x) \frac{\partial^{2} f}{\partial x \partial y}+(X-x)^{2} \frac{\partial^{2} f}{\partial x^{2}}=0$,

deren geometrischer Ort aus den beiden Tangenten des Doppelpunktes besteht. Für den Rückkehrpunkt bildet die linke Seite dieser Gleichung ein vollständiges Quadrat.

Ist die Kurve durch die beiden Gleichungen: $x=\varphi(t)$, $y=\psi(t)$ gegeben, so wird für den Doppelpunkt die Gleichung (5) der Tangente nicht unbestimmt, und das Kriterium des Doppelpunktes wird ein ganz anderes, als bisher. Der Doppelpunkt hat dann die Eigentümlichkeit, dass zwei ganz verschiedene Werte von $t$ gleiche Werte für $x$ und gleiche Werte für $y$ erzeugen müssen. Wir drücken diese Bedingung so aus:

$$
x=\varphi(t)=\varphi\left(t_{1}\right) ; \quad y=\psi(t)=\phi\left(t_{1}\right) .
$$

Die Lösungen beider Gleichungen sind die Parameter der Doppelpunkte. Werden die zusammengehörigen Werte $t$ und $t_{1}$ nach einander in (5) substituiert, so erhält man die beiden Tangenten des Doppelpunktes.

Rücken die Werte $t$ und $t_{1}$, welehe einem Doppelpunkt entsprechen, einander immer näher, so wird die Kurvenschleife immer kleiner und verschwindet zuletzt ganz, wenn $t$ mit $t_{1}$ zusammenfällt. Der Punkt wird dadurch ein Rückkehrpunkt; fuir ihn muss nicht nur $\varphi(t)=\varphi\left(t_{1}\right), \psi(t)=\psi\left(t_{1}\right)$ sein, sondern auch $t_{1}$ mit $t$ zusammenfallen. Nun sind für den Doppelpunkt die Gleichungen erfüllt:

$$
\frac{\varphi(t)-\varphi\left(t_{1}\right)}{t-t_{1}}=0, \quad \frac{\psi(t)-\psi\left(t_{1}\right)}{t-t_{1}}=0,
$$


wie eng auch die Kurvenschleife gemacht werde. Diese Gleichungen bleiben also auch für den Grenzfall bestehen, d. h. wir haben für den Rückkehrpunkt:

$$
\begin{array}{cc}
\lim \frac{\varphi(t)-\varphi\left(t_{1}\right)}{t-t_{1}}=0, & \lim \frac{\psi(t)-\psi\left(t_{1}\right)}{t-t_{1}}=0, \quad \text { oder } \\
\frac{d x}{d t}=0, & \frac{d y}{d t}=0 .
\end{array}
$$

Der Richtungskoefficient $\frac{d y}{d x}=\frac{d y}{d t}: \frac{d x}{d t}$ erscheint jetzt in der unbestimmten Form $\frac{0}{0}$ und hat den wahren Wert $\frac{d^{2} y}{d t^{2}}: \frac{d^{2} x}{d t^{2}}$. Die Gleichung der zusammenfallenden Tangenten eines Ruckkehrpunktes ist dann:

$$
\left\{(Y-y) \frac{d^{2} x}{d t^{2}}-(X-x) \frac{d^{2} y}{d t^{2}}\right\}^{2}=0 .
$$

Es wird nicht überflüssig sein, den Rückkehrpunkt noch aus einem andern Gesichtspunkt zu betrachten.

Wir nehmen auf einer stetig verlaufenden Kurve drei unendlich nahe liegende Punkte, die in der Anordnung 1, 2, 3 auf einander folgen, wenn man die Kurve von einem beliebig gewählten Anfangspunkte bis zu einem beliebigen Endpunkte durchläuft. Wir ziehen im Punkte 1 und im Punkte 2 die einseitigen Tangenten im Sinne des wachsenden Kurvenbogens, d.h. die geraden Verbindungslinien, welche von 1 uber 2 hinaus resp. von 2 über 3 hinaus einseitig verlaufen. Die Kurve heisst im Punkte 2 stetig gekrümmt, wenn diese einseitig gezogenen Tangenten einen unmessbar kleinen Winkel mit einander einschliessen. Dagegen findet in der Stetigkeit der Krümmung eine Unterbrechung statt, wenn die beiden Tangenten einen endlichen Winkel einschliessen. In diesem Falle wird der Punkt 2 eine Spitze genannt. Ein Rüekkehrpunkt ist eine Spitze, in welcher die Tangenten 1,2 und 2, 3 einen Winkel von $180^{\circ}$ mit einander bilden. Um sich davon zu überzeugen, braucht man nur den Rückkehrpunkt aus dem Doppelpunkte entstehen zu lassen. Nimmt man zuerst die Punkte 1, 2, 3 in endlieher Entfernung, so hat man jetzt die Punkte 1 und 3 
in den Doppelpunkt zu legen und den Punkt 2 an irgend eine Stelle der Kurvenschleife. Die Sehnen 1, 2 und 2, 3 fallen dann in dieselbe gerade Linie, aber ihre Richtungen sind entgegengesetzt. Sie schliessen einen Winkel von $180^{\circ} \mathrm{Grad}$ ein. Dies bleibt gültig, wie eng man auch die Schleife machen möge. Es gilt auch noch für den Grenzfall einer unendlich engen Schleife, d. h. für den Rückkehrpunkt. In diesem Falle ist die Sehne 1, 2 in die letzte Tungente vor dem Rückkehrpunkte ubergegangen, die Sehne 2, 3 in die erste Tangente nach dem Rückkehrpunkte. Beide Tangenten hat man einseitig im Sinne des wachsenden Bogens zu ziehen. Bei dieser Auffassung erbält man aus (14) die Gleichungen der beiden Tangenten einzeln genommen, indem man auf beiden Seiten der Gleichung (14) die Quadratwurzel auszieht, und der ausgezogenen Wurzel links einmal das Zeichen +, das andere Mal das Zeichen - vorsetzt.

15) Die Lemniskate: $\left(x^{2}+y^{2}\right)^{2}-2 a^{2}\left(x^{2}-y^{2}\right)=0$ hat in $x=0, y=0$ einen D.-P., dessen beide Tangenten: $Y= \pm X$ unter Winkeln von $45^{\circ}$ und $135^{\circ}$ die $X$-Achse durchschneiden, also zu einander senkrecht sind.

16.) Cissoide: $y^{2}(2 r-x)-x^{3}=0$; Rückkehrpunkt: $x=0$, $y=0 ; \mathrm{Tg} .: Y^{2}=0$.

17) Neilsche Parabel: $x^{3}-p y^{2}=0$; Ruickkehrpunkt: $x=0$, $y=0 ;$ Tg.: $Y^{2}=0$.

18) $t=x^{4}+a^{2} x^{2}-a^{2} y^{2}=0$; D.-P.: $x=0, y=0$; Tg.: $Y= \pm X$.

19) $f=x^{3}+x y^{2}-2 x^{2}-2 y+2=0$; Rliokkehrpunkt: $x=1$, $y=1 ;$ Tg: $(Y+X-2)^{2}=0$.

20) $f=y^{2}-x^{8}-x^{2}=0$; D.-P.: $x=y=0$; Tg.: $Y= \pm X$.

21) $f=x^{4}+y^{4}-2 a^{2} y^{2}-2 b^{2} x^{2}+b^{4}=0$; D.-P.: $x= \pm b$, $y=0 ; \mathrm{Tg} .: Y= \pm \frac{b}{a} \sqrt{2}(X+b) ; Y= \pm \frac{b \sqrt{2}}{a}(X-b)$.

22) $f=x^{4}-2 a y^{3}-3 a^{2} y^{2}-2 a^{2} x^{2}+a^{4}=0$; D.-P.: $x_{1}=a$, $y_{1}=0 ; \quad x_{2}=-a, y_{2}=0 ; x_{3}=0, y_{3}=-a ;$ Tg.: $Y_{1}= \pm \sqrt{\frac{4}{3}}\left(X_{1}-a\right) ; Y_{2}= \pm \sqrt{\frac{4}{3}}\left(X_{2}+a\right) ; Y_{3}= \pm \sqrt{\frac{2}{3}} X_{3}-a$. 
23) $f=(y-b)^{2}-(x-a)^{5}=0 ; \quad$ Rtickkehrpunkt: $x=a$, $y=b ; \quad \operatorname{Tg}:(Y-b)^{2}=0$.

24) $f=5 y^{2}+10 y-x^{3}+2 x^{2}+5=0$. Der Punkt $x=0$, $y=-1$ ist ein konjugierter Punkt.

25) $f=(y-x)^{2}-x^{3}=0$; Rïckkehrpunkt: $x=0, y=0$; Tg.: $Y=X$.

26) $f=a y^{2}-x^{3}+b x^{2}=0 ; x=0, y=0$ ein konjugierter Punkt.

27) $f=y^{2}-(2-x)^{2}(1-x)=0 ; x=2, y=0$ ein konjugierter Punkt.

28) Fusspunktlinie der Ellipse: $a x^{2}+b y^{2}-\left(x^{2}+y^{2}\right)^{2}=0$; $x=0, y=0$ ein konjugierter Punkt.

29) $y=a x+b \sqrt{\sin x-1}$ stellt ein System isolierter Punkte dar: $x_{1}=\frac{\pi}{2}, y_{1}=\frac{a \pi}{2} ; x_{2}=\frac{5 \pi}{2}, y_{2}=\frac{5 a \pi}{2}$, usw., die alle auf der Geraden $y=a x$ liegen.

30) Die Cykloide: $x=a(t-\sin t), y=a(1-\cos t) ; \varphi^{\prime}(t)$ $=a(1-\cos t)=0, \quad \psi^{\prime}(t)=a \sin t=0 . \quad$ Da beiden Gleichungen die Lösungen $t=0,2 \pi, 4 \pi$, usw. genügen, so sind $x_{1}=0, y_{1}=0 ; x_{2}=2 a \pi, y_{2}=0 ; x_{3}=4 a \pi$, $y_{3}=0$, usw. Rückkehrpunkte. Die Tangenten sind senkrecht zur Achse.

31) Die Evolute der Ellipse:

$$
x=\frac{a^{2}-b^{2}}{a} \cos ^{3} t, \quad y=-\frac{a^{2}-b^{2}}{b} \sin ^{3} t
$$

hat 4 Rückkehrpunkte, welche den Parametern $0, \frac{\pi}{2}$, $\pi, \frac{3 \pi}{2}$ entsprechen.

§ 3. Krümmungskreis und Evolute.

Wählt man auf einer Kurve drei einander nahe gelegene Punkte 1, 2, 3, zieht die Sehnen 1, 2 und 2, 3, und errichtet in der Mitte beider je eine Senkrechte, so ist der Schnittpunkt dieser Senkrechten der Mittelpunkt eines Kreises, welcher durch die drei Punkte geht. Indem diese einander 
immer näher rücken, werden die Sehnen immer kürzer und im Grenzfalle zu Kurvenelementen, welche zugleich auch dem Kreis angehören. Die beiden Senkrechten werden dabei zu zwei unendlich nahe gelegenen Normalen, und der Kreis selbst wird zum Krümmungskreis. Die Stärke der Krümmung einer Kurve in einem bestimmten Punkt wird gemessen durch die Grösse der Krümmung des dem betreffenden Punkt zugehörigen Krümmungskreises, und da diese mit wachsendem Halbmesser $=\varrho$ abnimmt, so gilt der reciproke Wert dieses Halbmessers, nämlich $\frac{1}{\varrho}$, als Ausdruck für das Mass der Krümmung. Wir beginnen mit der Bestimmung des Krümmungsmittelpunktes für die Gleichungsform: $x=\varphi(t), y=\psi(t)$, legen dureh zwei vorerst noch endlich entfernte Punkte $x, y$ und $x_{1}, y_{1}$ zwei Normalen und bestimmen ihren Schnittpunkt. Die Gleichungen dieser Normalen sind:

$$
\begin{gathered}
(X-x) \frac{d x}{d t}+(Y-y) \frac{d y}{d t}=0 \\
\left(X-x_{1}\right) \frac{d x_{1}}{d t_{1}}+\left(Y-y_{1}\right) \frac{d y_{1}}{d t_{1}}=0 .
\end{gathered}
$$

Die Koordinaten $X$ und $Y$ ihres Schnittpunktes genügen beiden Gleichungen. Die Gleichung (15) wird allgemein befriedigt, wenn man unter Benutzung eines noch unbestimmten Koefficienten $m$ ansetzt:

$$
X=x-m \frac{d y}{d t}, \quad Y=y+m \frac{d x}{d t} .
$$

Diese Ausdrücke für $X$ und $Y$ sind in (16) einzuführen; so erhält man eine Gleichung für $m$ :

$$
\begin{gathered}
\left(x-m \frac{d y}{d t}-x_{1}\right) \frac{d x_{1}}{d t_{1}}+\left(y+m \frac{d x}{d t}-y_{1}\right) \frac{d y_{1}}{d t_{1}}=0 \\
m=\frac{\left(x-x_{1}\right) \frac{d x_{1}}{d t_{1}}+\left(y-y_{1}\right) \frac{d y_{1}}{d t_{1}}}{\frac{d y}{d t} \frac{d x_{1}}{d t_{1}}-\frac{d x}{d t} \frac{d y_{1}}{d t_{1}}} .
\end{gathered}
$$


Wird dieses $m$ in (17) substituiert, so gewinnen wir den Schnittpunkt zweier endlich entfernten Normalen. Da aber jener Schnittpunkt unter der Voraussetzung gefunden werden soll, dass die Normalen unendlich wenig von einander entfernt sind, so ist in (18) ein Grenzübergang auszuführen und dieser Ausdruck zunächst entsprechend umzuformen.

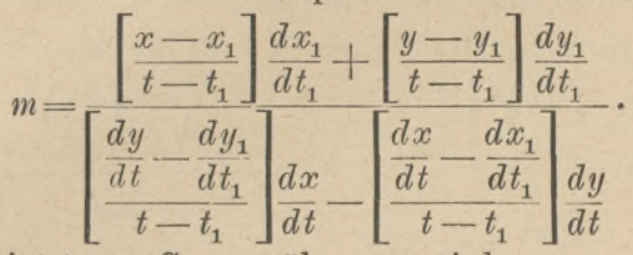

Geht man jetzt zur Grenze über, so wird:

$$
m=\frac{\left(\frac{d x}{d t}\right)^{2}+\left(\frac{d y}{d t}\right)^{2}}{\frac{d x}{d t} \frac{d^{2} y}{d t^{2}}-\frac{d y}{d t} \frac{d^{2} x}{d t^{2}}} .
$$

Nun werden die Koordinaten des Krümmungsmittelpunktes gefunden, wenn man dieses $m$ in (17) substituiert:

$$
\begin{aligned}
& X-x-\frac{\left[\left(\frac{d x}{d t}\right)^{2}+\left(\frac{d y}{d t}\right)^{2}\right] \frac{d y}{d t}}{\frac{d x}{d t} \frac{d^{2} y}{d t^{2}}-\frac{d y}{d t} \frac{d^{2} x}{d t^{2}}} \\
& Y=y+\frac{\left[\left(\frac{d x}{d t}\right)^{2}+\left(\frac{d y}{d t}\right)^{2}\right] \frac{d x}{d t}}{\frac{d x}{d t} \frac{d^{2} y}{d t^{2}}-\frac{d y}{d t} \frac{d^{2} x}{d t^{2}}} .
\end{aligned}
$$

Die Entfernung der beiden Punkte $X, Y$ und $x, y$ d. h. der Krümmungshalbmesser, wird nach folgenden Formeln berechnet:

$$
\begin{aligned}
\varrho & =\sqrt{(X-x)^{2}+(Y-y)^{2}}=m \sqrt{\left(\frac{d y}{d t}\right)^{2}+\left(\frac{d x}{d t}\right)^{2}} \\
& =\frac{\sqrt{\left[\left(\frac{d x}{d t}\right)^{2}+\left(\frac{d y}{d t}\right)^{2}\right]^{3}}}{\frac{d x}{d t} \frac{d^{2} y}{d t^{2}}-\frac{d y}{d t} \frac{d^{2} x}{d t^{2}}} .
\end{aligned}
$$


Geht in dieser Entwickelung $t$ tuber in $x$, so wird $y=\psi(x)$ oder $=f(x), \frac{d y}{d t}=\frac{d y}{d x}, \frac{d x}{d t}=1, \frac{d^{2} x}{d t^{2}}=0$, und daher:

$$
\begin{gathered}
m=\frac{1+\left(\frac{d y}{d x}\right)^{2}}{\frac{d^{2} y}{d x^{2}}} . \\
X=x-\frac{\left[1+\left(\frac{d y}{d x}\right)^{2}\right] \frac{d y}{d x}}{\frac{d^{2} y}{d x^{2}}}, \quad Y=y+\frac{1+\left(\frac{d y}{d x}\right)^{2}}{\frac{d^{2} y}{d x^{2}}} . \\
\varrho=\frac{\sqrt{\left[1+\left(\frac{d y}{d x}\right)^{2}\right]^{3}}}{\frac{d^{2} y}{d x^{2}}} .
\end{gathered}
$$

Ist die Gleichung der Kurve in Polarkoordinaten gegeben und von der Form: $r=f(t)$, so ist $x=r \cos t, y=r \sin t$. Die Formeln (21) und (22) gehen dann in folgende tiber:

$$
\begin{gathered}
X=x-\frac{\left[\left(\frac{d r}{d t}\right)^{2}+r^{2}\right]\left[\frac{d r}{d t} \sin t+r \cos t\right]}{r^{2}+2\left(\frac{d r}{d t}\right)^{2}-r \frac{d^{2} r}{d t^{2}}} \\
Y=y+\frac{\left[\left(\frac{d r}{d t}\right)^{2}+r^{2}\right]\left[\frac{d r}{d t} \cos t-r \sin t\right]}{r^{2}+2\left(\frac{d r}{d t}\right)^{2}-r \frac{d^{2} r}{d t^{2}}} \\
\varrho=\frac{\sqrt{\left[\left(\frac{d r}{d t}\right)^{2}+r^{2}\right]^{3}}}{r^{2}+2\left(\frac{d r}{d t}\right)^{2}-r \frac{d^{2} r}{d t^{2}}} .
\end{gathered}
$$

Wenn endlich die Kurvengleichung in $\operatorname{der}$ Form $f(x, y)=0$ vorliegt, so werden nach (7) zwei zunächst wieder endlich 
von einander entfernte Normalen der Punkte $x, y$ und $x_{1}, y_{1}$ durch folgende Gleichungen ausgedrückt:

$$
\begin{aligned}
& (X-x) \frac{\partial f}{\partial y}-(Y-y) \frac{\partial f}{\partial x}=0 \\
& \left(X-x_{1}\right) \frac{\partial f}{\partial y_{1}}-\left(Y-y_{1}\right) \frac{\partial f}{\partial x_{1}}=0 .
\end{aligned}
$$

Zur Bestimmung der Schnittpunktskoordinaten $X, Y$ setzt man aus (29), ähnlich wie oben bei (17):

substituiert dann:

$$
\frac{X-x}{\frac{\partial f}{\partial x}}=\frac{Y-y}{\frac{\partial f}{\partial y}}=m_{1},
$$

$$
X=x+m_{1} \frac{\partial f}{\partial x}, \quad Y=y+m_{1} \frac{\partial f}{\partial y}
$$

in (30) und erhält:

$$
\begin{gathered}
\left(x-x_{1}+m_{1} \frac{\partial f}{\partial x}\right) \frac{\partial f}{\partial y_{1}}-\left(y-y_{1}+m_{1} \frac{\partial f}{\partial y}\right) \frac{\partial f}{\partial x_{j}}=0 \\
m_{1}=\frac{\left(x_{1}-x\right) \frac{\partial f}{\partial y_{1}}-\left(y_{1}-y\right) \frac{\partial f}{\partial x_{1}}}{\frac{\partial f}{\partial x} \frac{\partial f}{\partial y_{1}}-\frac{\partial f}{\partial y} \frac{\partial f}{\partial x_{1}}} \\
\left.m_{1}=\frac{\frac{\partial f}{\partial y_{1}}-\left(\frac{y_{1}-y}{x_{1}-x}\right) \frac{\partial f}{\partial x_{1}}}{\frac{\partial f}{\partial x}\left[\frac{\partial f}{\frac{\partial y_{1}}{x_{1}}-\frac{\partial f}{\partial y}}\right]-\left[\frac{\partial f}{\partial x_{1}}-\frac{\partial f}{\partial x}\right]} . \frac{\partial f}{x_{1}-x}\right]
\end{gathered}
$$

Rücken beide Normalen unendlich nahe zusammen, so wird ihr Schnittpunkt zum Krümmungsmittelpunkt. Hierbei sind in dem Wert von $m_{1}$ folgende Grenzubergänge auszuftihren:

$$
\begin{gathered}
\frac{\partial f}{\partial x_{1}} \text { wird }=\frac{\partial f}{\partial x} ; \quad \frac{\partial f}{\partial y_{1}}=\frac{\partial f}{\partial y} ; \quad \lim \frac{y_{1}-y}{x_{1}-x}=\frac{d y}{d x} ; \\
\lim \left[\frac{\frac{\partial f}{y_{1}}-\frac{\partial f}{\partial y}}{x_{1}-x}\right]=\frac{d\left(\frac{\partial f}{\partial y}\right)}{d x}=\frac{\partial^{2} f}{\partial y \partial x}+\frac{\partial^{2} f}{\partial y^{2}} \frac{d y}{d x}
\end{gathered}
$$




$$
\lim \left[\frac{\frac{\partial f}{\partial x_{1}}-\frac{\partial f}{\partial x}}{x_{1}-x}\right]=\frac{d\left(\frac{\partial f}{\partial x}\right)}{d x}=\frac{\partial^{2} f}{\partial x^{2}}+\frac{\partial^{2} f}{\partial x \partial y} \frac{d y}{d x} \text {. Werden }
$$

diese Werte in (32) eingeftuhrt und $-\frac{\partial f}{\partial x}: \frac{\partial f}{\partial y}$ für $\frac{d y}{d x}$ gesetzt, so erhält man denjenigen Wert von $m_{1}$, welcher in (31) substituiert, uns $X$ und $Y$ als Koordinaten des Krümmungsmittelpunktes giebt. Man erhält:

$$
\begin{gathered}
m_{1}=\frac{\left(\frac{\partial f}{\partial x}\right)^{2}+\left(\frac{\partial f}{\partial y}\right)^{2}}{-\frac{\partial^{2} f}{\partial x^{2}}\left(\frac{\partial f}{\partial y}\right)^{2}+2 \frac{\partial^{2} f}{\partial x \partial y} \frac{\partial f}{\partial x} \frac{\partial f}{\partial y}-\frac{\partial^{2} f}{\partial y^{2}}\left(\frac{\partial f}{\partial x}\right)^{2}} \\
\varrho= \pm m_{1} \sqrt{\left(\frac{\partial f}{\partial x}\right)^{2}+\left(\frac{\partial f}{\partial y}\right)^{2}} \\
=\frac{ \pm \sqrt{\left.\left[\frac{\partial f}{\partial x}\right)^{2}+\left(\frac{\partial f}{\partial y}\right)^{2}\right]^{8}}}{-\frac{\partial^{2} f}{\partial x^{2}}\left(\frac{\partial f}{\partial y}\right)^{2}+2 \frac{\partial^{2} f}{\partial x \partial y} \frac{\partial f}{\partial x} \frac{\partial f}{\partial y}-\frac{\partial^{2} f}{\partial y^{2}}\left(\frac{\partial f}{\partial x}\right)^{2}}
\end{gathered}
$$

Man kann zu der Formel (34) auch dadurch gelangen, dass man von (23) und (25) ausgeht und dort einsetzt:

$$
\frac{\frac{d y}{d x}=-\frac{\partial f}{\partial x}: \frac{\partial f}{\partial y}}{d x^{2}}=\frac{-\frac{\partial^{2} f}{\partial x^{2}}\left(\frac{\partial f}{\partial y}\right)^{2}+2 \frac{\partial^{2} f}{\partial x \partial y} \frac{\partial f}{\partial x} \frac{\partial f}{\partial y}-\frac{\partial^{2} f}{\partial y^{2}}\left(\frac{\partial f}{\partial x}\right)^{2}}{\left(\frac{\partial f}{\partial y}\right)^{3}} .
$$

Hier ist leicht zu erkennen, dass $m=m_{1} \frac{\partial f}{\partial y}$ ist. Es ist also in (34) das positive oder das negative Vorzeichen $\mathrm{zu}$ wählen, je nachdem $\frac{\partial f}{\partial y}$ positiv oder negativ ist. Dann stimmen die Werte von $\varrho$ aus (25) und (34) vollständig uberein.

Wir bezeichnen mit $v$ den Winkel, den der Krlimmungshalbmesser in der Richtung von der Kurve nach dem Krlim- 
mungsmittelpunkte hin mit der Richtung der wachsenden $x$ einschliesst. Dann ist:

$\cos v=\frac{X-x}{\sqrt{(X-x)^{2}+(Y-y)^{2}}}, \sin v=\frac{Y-y}{\sqrt{(X-x)^{2}+(Y-y)^{2}}}$.

Durch diese Gleichungen ist eindeutig entschieden, in welcher Richtung vom Kurvenpunkte $(x, y)$ aus man den Krümmungshalbmesser auf der Normale abzutragen hat. Man darf deshalb bei der Berechnung des Krümmungshalbmessers sich auf den absoluten Zahlwert beschränken.

Wir unterscheiden die konkave und die konvexe Seite der Kurve. An der Stelle, wo dieselbe drei unendlich nahe gelegene Punkte mit ihrem Krümmungskreise gemein hat, kehrt sie dem zugehörigen Krümmungsmittelpunkte ihre konkave Seite zu. Die konvexe Seite ist die abgekehrte.

Jedem Punkt der gegebenen Kurve entspricht ein bestimmter Krümmungsmittelpunkt. Der geometrische Ort aller Krümmungsmittelpunkte wird Evolute genannt. Die beiden Gleichungen für $X$ und $Y$ bestimmen zusammen mit der Gleiohung der gegebenen Kurve die Gleiohung dieser Evolute.

Die Elemente des Krümmungskreises für die folgenden Kurven zu bestimmen:

32) Der Kreis: $x^{2}+y^{2}-r^{2}=0 ; m_{1}=-\frac{1}{2} ; X=0, Y=0$; $\varrho=\mp r$. Der K.-K. fällt ganz mit dem gegebenen Kreis zusammen.

33) Die Ellipse: $x=a \cos t, y=b \sin t$;

$m=\frac{a^{2}-\left(a^{2}-b^{2}\right) \cos ^{2} t}{a b} ; X=\frac{a^{2}-b^{2}}{a} \cos ^{8} t, Y=\frac{b^{2}-a^{2}}{b} \sin ^{8} t$.

Wird aus beiden Gleichungen die Variabele $t$ eliminiert, so erhält man als Gleichung der Evolute: $\sqrt[3]{b^{2} Y^{2}}$ $+\sqrt[3]{a^{2} X^{2}}=\sqrt[3]{\left(a^{2}-b^{2}\right)^{2}}$. Ferner ist $X=\frac{a^{2}-b^{2}}{a^{4}} x^{3}$, $Y=-\frac{a^{2}-b^{2}}{b^{4}} y^{3} ; \varrho=\frac{\left\{a^{2}-\left(a^{2}-b^{2}\right) \cos ^{2} t\right\}^{\frac{3}{2}}}{a b}$. 
34) Die Hyperbel: $\frac{x^{2}}{a^{2}}-\frac{y^{2}}{b^{2}}=1$;

$$
\begin{aligned}
& m_{1}=\left(\frac{x^{2}}{a^{4}}+\frac{y^{2}}{b^{4}}\right) \frac{a^{2} b^{2}}{2} ; \\
& X=\frac{a^{2}+b^{2}}{a^{4}} x^{3}, \quad Y=-\frac{a^{2}+b^{2}}{b^{4}} y^{3} ; \\
& \varrho= \pm a^{2} b^{2}\left(\frac{x^{2}}{a^{4}}+\frac{y^{2}}{b^{4}}\right)^{\frac{3}{2}} .
\end{aligned}
$$

35) Die Parabel: $y^{2}-2 p x=0 ; m_{1}=-\frac{y^{2}+p^{2}}{2 p^{2}} ; \quad X=3 x+p$, $Y=-\frac{y^{3}}{p^{2}} ; \quad x=\frac{X-p}{3}, \quad y^{2}=\sqrt[3]{p^{4} Y^{2}}$ in die Parabelgleichung substituiert, giebt als Evolute: $Y^{2}=\frac{8}{27 p}(X-p)^{3}$. Man sieht, dass diese Evolute die Neilsche Parabel ist, (S. 180). Weiter ist $\varrho=\mp \frac{1}{p^{2}} \sqrt{\left(p^{2}+y^{2}\right)^{3}}$.

36) Die Cissoide: $x^{3}-y^{2}(2 r-x)=0 ; m_{1}=-\frac{8 r-3 x}{6 x(2 r-x)}$; $X=\frac{-r x(12 r-5 x)}{3(2 r-x)^{2}}, Y=\frac{8 r \sqrt{x}}{3 \sqrt{(2 r-x)}} ; \varrho=\mp \frac{r \sqrt{x(8 r-3 x)^{3}}}{3(2 r-x)^{2}}$.

37) Die Kettenlinie: $y=\frac{a}{2}\left(e^{\frac{x}{a}}+e^{-\frac{x}{a}}\right) ; \quad m=y$; $X=x-\frac{a}{4}\left(e^{\frac{2 x}{a}}-e^{-\frac{2 x}{a}}\right), Y=y+\frac{a}{2}\left(e^{\frac{x}{a}}+e^{-\frac{x}{a}}\right)=2 y ;$ $\varrho=\frac{a}{4}\left(e^{\frac{x}{a}}+e^{-\frac{x}{a}}\right)^{2}=\frac{y^{2}}{a}$.

Das Stlick der Normale zwischen der Kurve und der Abscissenachse wird bei jeder Kurve nach der Formel berechnet: $N=y \sqrt{1+\left(\frac{d y}{d x}\right)^{2}}$. Da man hiernach bei der Kettenlinie $N=\frac{y^{2}}{a}$ findet, so erkennt man, dass die Normale dem Krümmungshalbmesser gleich ist. Sie liegen von der Kurve aus einander entgegengesetzt. 
38) Die Cykloide: $x=a(t-\sin t), y=a(1-\cos t) ; m=-2$; $X=a(t+\sin t), Y=-a(1-\cos t)=-y$. Beide Gleichungen repräsentieren zusammen die Evolute der Cykloide. Setzt man $t_{1}+\pi$ statt $t$, so wird $X-a \pi=a\left(t_{1}-\sin t_{1}\right)$, $Y+2 a=a\left(1-\cos t_{1}\right)$; wird jetzt $X-a \pi$ durch $X_{1}$, $Y+2 a$ durch $Y_{1}$ ersetzt, was einer Verschiebung des Anfangspunktes um $a \pi$ und $-2 a$ entspricht, so erhält man als Gleichungen der Evolute:

$$
X_{1}=a\left(t_{1}-\sin t_{1}\right), \quad Y_{1}=a\left(1-\cos t_{1}\right),
$$

und daraus folgt, dass die Evolute eine kongruente Cykloide ist, die sich nur hinsichtlich der Lage dadurch von der gegebenen unterscheidet, dass ibr Anfangspunkt um $a \pi$ und $-2 a$ verschoben ist. Wir finden $\varrho=-4 a \sin \frac{t}{2}$, und da die Normale $=2 a \sin \frac{t}{2}$ gefunden wird, so ist der Krümmungshalbmesser der doppelten Normale gleich. Sie liegen von der Kurve aus nach derselben Seite. Um den Krümmungsmittelpunkt zu konstruieren, hat man die Normale nur um sich selbst zu verlängern.

39) Die Epicykloide: $x=(a+r) \cos t-a \cos \left(\frac{a+r}{a}\right) t$, $y=(a+r) \sin t-a \sin \left(\frac{a+r}{a}\right) t ; \quad m=\frac{2 a}{r+2 a} ;$ $X=\frac{r}{r+2 a}\left[(a+r) \cos t+a \cos \left(\frac{a+r}{a}\right) t\right] ;$
$Y=\frac{r}{r+2 a}\left[(a+r) \sin t+a \sin \left(\frac{a+r}{a}\right) t\right] ;$ $\varrho=\frac{4 a(r+a)}{r+2 a} \sin \frac{r}{2 a} t$.

Der Kr.-Mittelpunkt wird auf folgende Weise konstruiert: Man zieht (Fig. 14) den Durchmesser $M G$, verbindet $G$ mit $A$ und verlängert die Normale $M B$; der Sehnittpunkt $K$ ist der gesuchte Kr.-Mittelpunkt und 
$K M$ der Kr.-Halbmesser. Da $G N$ parallel und gleich $B M$ ist, so haben wir

$$
\begin{gathered}
K B=\frac{r}{r+2 a} G N=\frac{r}{r+2 a} B M, \text { und } \\
K M=\left(1+\frac{r}{r+2 a}\right) B M=\frac{2(a+r)}{2 a+r} B M \\
=\frac{2(a+r)}{2 a+r} 2 a \sin \frac{t_{1}}{2}=\frac{4 a(a+r)}{2 a+r} \sin \frac{r t}{2 a}=\varrho .
\end{gathered}
$$

40) Die Lemniskate: $r=a \sqrt{\cos 2 t}$. Es ist $m=\frac{1}{3}$;

$$
X=\frac{2 a \cos ^{3} t}{3 \sqrt{\cos 2 t}}, \quad Y=-\frac{2 a \sin ^{3} t}{3 \sqrt{\cos 2 t}} ; \varrho=\frac{a}{3 \sqrt{\cos 2 t}}=\frac{a^{2}}{3 r} .
$$

Da $x=r \cos t, y=r \sin t$, so ist $x^{2}+y^{2}=a^{2} \cos 2 t$, oder $\cos 2 t=\frac{x^{2}+y^{2}}{a^{2}}, \varrho=\frac{a^{2}}{3 \sqrt{x^{2}+y^{2}}}$. Driickt man $\sin ^{2} t$ und $\cos ^{2} t$ durch die Koordinaten des Kr.-Mittelpunktes aus, so gewinnt man mit Hilfe der beiden Relationen: $\cos ^{2} t+\sin ^{2} t=1$ und $\cos ^{2} t-\sin ^{2} t=\cos 2 t$ die Gleichung der Evolute: $3\left(X^{\frac{2}{3}}+Y^{\frac{2}{3}}\right)\left(X^{\frac{2}{3}}-Y^{\frac{2}{3}}\right)^{\frac{1}{2}}=2 a$.

41) Die logarithmische Spirale: $r=a^{t}$. Hier ist $m=1$; $X=-a^{t} \lg a \sin t, Y=a^{t} \lg a \cos t ; \varrho=a^{t} \sqrt{1+(\lg a)^{2}}$. Um die Polargleichung der Evolute zu erhalten, setze man den Leitstrahl $R=\sqrt{X^{2}+Y^{2}}=a^{t} \lg a$. Wird $\lg a=a^{\bullet}$ gesetzt, so ist $R=a^{t+\bullet}$, d. h. die Evolute dieser Spirale ist eine gleiche Spirale, welche nur um den Winkel $\varepsilon$ gedreht ist.

\section{§ 4. Die Wende- oder Inflexionspunkte.}

Liegen drei unendlich nahe Punkte einer Kurve in einer geraden Linie, so wird der Krümmungshalbmesser an dieser Stelle unendlich gross. Es seien $x$ und $y$ die Koordinaten dieser Stelle. Dann werden hier auch $X-x$ und $Y-y$ unendlich gross. Ändern diese Differenzen dabei gleichzeitig ihr Vorzeichen, wenn man, die Kurve stetig durchlaufend, durch den Punkt $(x, y)$ hindurchgeht, so ändert die Kurve an dieser 
Stelle die Art ihrer Krümmung. Ihre konkave Seite geht in die konvexe ubber und umgekehrt. Ein solcher Punkt $(x, y)$ wird ein Wendepunkt oder Inflexionspunkt genannt.

Wir verbinden die Bedingungsgleichung für das Unendlichwerden des Krümmungshalbmessers mit der Gleichung der Kurve und erhalten als Lösungen beider Gleichungen die Koordinaten aller Punkte, die Wendepunkte sein können. Sie sind wirklich Wendepunkte, wenn in ihnen der Vorzeichenwechsel der Differenzen $X-x$ und $Y-y$ eintritt.

Ist $f(x, y)=0$ die gegebene Gleichung, so wird nach (34) $\varrho$ unendlich, wenn:

$$
-\frac{\partial^{2} f}{\partial x^{2}}\left(\frac{\partial f}{\partial y}\right)^{2}+2 \frac{\partial^{2} f}{\partial x \partial y} \frac{\partial f}{\partial x} \frac{\partial f}{\partial y}-\frac{\partial^{2} f}{\partial y^{2}}\left(\frac{\partial f}{\partial x}\right)^{2}=0 .
$$

Die linke Seite dieser Gleichung lässt sich in Form einer Determinante schreiben. Alsdann lautet die Bedingungsgleiohung:

$$
\Delta=\left|\begin{array}{ccc}
\frac{\partial^{2} f}{\partial x^{2}} & \frac{\partial^{2} f}{\partial x \partial y} & \frac{\partial f}{\partial x} \\
\frac{\partial^{2} f}{\partial x \partial y} & \frac{\partial^{2} f}{\partial y^{2}} & \frac{\partial f}{\partial y} \\
\frac{\partial f}{\partial x} & \frac{\partial f}{\partial y} & 0
\end{array}\right|=0
$$

Wird die dritte Vertikalreihe mit $(n-1)$ multipliziert, die ganze Determinante durch $(n-1)$ dividiert, und 0 durch $n f$ ersetzt, so erhält man:

$$
\Delta=\frac{1}{n-1}\left|\begin{array}{ccc}
\frac{\partial^{2} f}{\partial x^{2}} & \frac{\partial^{2} f}{\partial x \partial y} & (n-1) \frac{\partial f}{\partial x} \\
\frac{\partial^{2} f}{\partial x \partial y} & \frac{\partial^{2} f}{\partial y^{2}} & (n-1) \frac{\partial f}{\partial y} \\
\frac{\partial f}{\partial x} & \frac{\partial f}{\partial y} & n f
\end{array}\right|=0
$$

In der Determinante darf man die mit $x$ multiplizierten Elemente der ersten und die mit $y$ multiplizierten Elemente der zweiten Vertikalreihe von denen der dritten Reihe subtra- 
hieren. Dadurch ergiebt sich für $(n-1) \Delta$ die Determinantenform

$$
\left|\begin{array}{ccc}
\frac{\partial^{2} f}{\partial x^{2}} & \frac{\partial^{2} f}{\partial x \partial y} & (n-1) \frac{\partial f}{\partial x}-x \frac{\partial^{2} f}{\partial x^{2}}-y \frac{\partial^{2} f}{\partial x \partial y} \\
\frac{\partial^{2} f}{\partial x \partial y} & \frac{\partial^{2} f}{\partial y^{2}} & (n-1) \frac{\partial f}{\partial y}-x \frac{\partial^{2} f}{\partial x \partial y}-y \frac{\partial^{2} f}{\partial y^{2}} \\
\frac{\partial f}{\partial x} & \frac{\partial f}{\partial y} & n f \quad-x \frac{\partial f}{\partial x}-y \frac{\partial f}{\partial y}
\end{array}\right|
$$

Wird jetzt die gegebene Funktion $f(x, y)=0$ in die homogene Funktion $f(x, y, z)=0$ umgewandelt, so kann der Lehrsatz von Euler: $x \frac{\partial f}{\partial x}+y \frac{\partial f}{\partial y}+z \frac{\partial f}{\partial z}=n f$, wobei $n$ den Grad der Funktion anzeigt, zur Anwendung kommen. Da ferner $\frac{\partial f}{\partial x}, \frac{\partial f}{\partial y}, \frac{\partial f}{\partial z}$ wieder lomogene Funktionen sein werden, und zwar vom $(n-1)^{\text {ten }}$ Grad, so ist nach demselben Satz:

$$
\begin{aligned}
& x \frac{\partial^{2} f}{\partial x^{2}}+y \frac{\partial^{2} f}{\partial x \partial y}+z \frac{\partial^{2} f}{\partial x \partial z}=(n-1) \frac{\partial f}{\partial x} \\
& x \frac{\partial^{2} f}{\partial y \partial x}+y \frac{\partial^{2} f}{\partial y^{2}}+z \frac{\partial^{2} f}{\partial y \partial z}=(n-1) \frac{\partial f}{\partial y} \\
& x \frac{\partial^{2} f}{\partial z} \frac{\partial^{2} f}{\partial x}+y \frac{\partial^{2} f}{\partial z \partial y}+z \frac{\partial^{2} f}{\partial z^{2}}=(n-1) \frac{\partial f}{\partial z} .
\end{aligned}
$$

Mittelst dieser Relationen bringt man (37) auf die Form:

$$
\Delta=\frac{1}{n-1}\left|\begin{array}{ccc}
\frac{\partial^{2} f}{\partial x^{2}} & \frac{\partial^{2} f}{\partial x \partial y} & z \frac{\partial^{2} f}{\partial x \partial z} \\
\frac{\partial^{2} f}{\partial y \partial x} & \frac{\partial^{2} f}{\partial y^{2}} & z \frac{\partial^{2} f}{\partial y \partial z} \\
\frac{\partial f}{\partial x} & \frac{\partial f}{\partial x} & z \frac{\partial f}{\partial z}
\end{array}\right|=0
$$

Wird in dieser Determinante wieder $z=1$ gesetzt, so bildet sie die Bedingungsgleichung für das Unendlichwerden des Kr.-Halbmessers. Sie gebt leicht in folgende uber: 


$$
\Delta=\frac{1}{(n-1)^{2}}\left|\begin{array}{ccc}
\frac{\partial^{2} f}{\partial x^{2}} & \frac{\partial^{2} f}{\partial x \partial y} & \frac{\partial^{2} f}{\partial x \partial z} \\
\frac{\partial^{2} f}{\partial y \partial x} & \frac{\partial^{2} f}{\partial y^{2}} & \frac{\partial^{2} f}{\partial y \partial z} \\
(n-1) \frac{\partial f}{\partial x} & (n-1) \frac{\partial f}{\partial y} & (n-1) \frac{\partial f}{\partial z}
\end{array}\right|=0 .
$$

Subtrahiert man von den Elementen der dritten Horizontalreihe die mit $x$ multiplizierten Elemente der ersten und die mit $y$ multiplizierten Elemente der zweiten Reihe, substituiert dann für die Elemente der dritten Reihe die Werte aus (38) und setzt dabei $z=1$, so erhält man:

$$
\Delta_{1}=(n-1)^{2} \Delta=\left|\begin{array}{ccc}
\frac{\partial^{2} f}{\partial x^{2}} & \frac{\partial^{2} f}{\partial x \partial y} & \frac{\partial^{2} f}{\partial x \partial z} \\
\frac{\partial^{2} f}{\partial y \partial x} & \frac{\partial^{2} f}{\partial y^{2}} & \frac{\partial^{2} f}{\partial y \partial z} \\
\frac{\partial^{2} f}{\partial z \partial x} & \frac{\partial^{2} f}{\partial z \partial y} & \frac{\partial^{2} f}{\partial z^{2}}
\end{array}\right|=0 .
$$

Die Schnittpunkte der beiden Kurven $f=0$ und $\Delta=0$ oder $\Delta_{1}=0$ geben die Wendepunkte der Kurve. Ist $f=0$ vom $n^{\text {ten }}$ Grad, so muss die Inflexions-Determinante vom $3(n-2)^{\text {ten }}$ Grad sein. Beide Gleichungen lassen daher im allgemeinen $3 n(n-2)$ Lösungen $\mathrm{zu}$, und die Kurve hat eben so viele Wendepunkte. Kurven des zweiten Grades haben demnach keine Wendepunkte, vgl. Aufgabe 48).

Ist die Kurve durch die beiden Gleichungen gegeben: $x=\varphi(t), y=\psi(t)$, so wird der Krümmungshalbmesser nach (22) unendlich, wenn:

$$
\frac{d x}{d t} \cdot \frac{d^{2} y}{d t^{2}}-\frac{d y}{d t} \cdot \frac{d^{2} x}{d t^{2}}=0,
$$

und aus dieser Gleichung entnehmen wir die Parameter der Wendepunkte.

Für die Form $y=f(x)$ wird nach (23) der Krümmungshalbmesser unendlich, wenn:

$$
\frac{d^{2} y}{d x^{2}}=0
$$


Die reellen, im Endlichen gelegenen Wendepunkte folgender Kurven zu finden:

42) $f=x^{8}+y^{3}+6 a x y+1=0$.

Homogene Form: $x^{3}+y^{3}+6 a x y z+z^{3}=0$.

$$
\Delta_{1}=6^{3}\left|\begin{array}{ccc}
x & a z & a y \\
a z & y & a x \\
a y & a x & z
\end{array}\right|
$$

$\frac{1}{6^{3}} \Delta_{1}=x y z\left(1+2 a^{3}\right)-a^{2}\left(x^{3}+y^{3}+z^{3}\right)=0 ; z=1$ giebt: $x^{3}+y^{8}+1-\frac{1+2 a^{8}}{a^{2}} x y=0$. Aus dieser Gleichung und $f=0$ finden wir: $x=0, y=-1 ; x=-1, y=0$ als Koordinaten der Wendepunkte.

43) $f=x^{3}-3 a x^{2}+c^{2} y=0$.

Homogene Form: $x^{3}-3 a x^{2} z+c^{2} y z^{2}=0$.

$$
\Delta_{1}=\left|\begin{array}{ccc}
6(x-a z) & 0 & -6 a x \\
0 & 0 & 2 c^{2} z \\
-6 a x & 2 c^{2} z & 2 c^{2} y
\end{array}\right|
$$

$-\Delta_{1}=24(x-a) c^{4}=0$. Aus $\Delta_{1}=0$ und $f=0$ findet $\operatorname{man} x=a, y=\frac{2 a^{8}}{c^{2}}$ als Koordinaten eines Wendepunktes.

44) $f=x^{2} y-x+y=0$, oder $x^{2} y-x z^{2}+y z^{2}=0$.

Bedingung: $x^{8}-x^{2} y-2 x-y=0$. Wendepunkte:

$x=0, y=0 ; x= \pm \sqrt{3}, y= \pm \frac{\sqrt{3}}{4}$.

45) $f=(a-x)^{5}-y=0$. Bedingung: $(a-x)^{8}=0$. Wendepunkt: $x=a, y=0$.

46) $f=x^{4}-a^{2} x^{2}+a^{8} y=0$. Wendepunkte: $x= \pm \frac{a}{\sqrt{6}}, y=\frac{5 a}{36}$.

47) $f=y-x^{3}=0$. Wendepunkt: $x=0, y=0$.

48) Die Wendepunkte eines Kegelschnitts sollen aus der allgemeinen Gleichung:

$$
A y^{2}+2 B x y+C x^{2}+2 D y+2 E x+F=0
$$

gefunden werden. 


$$
\Delta=\left|\begin{array}{lll}
2 C & 2 B & 2 E \\
2 B & 2 A & 2 D \\
2 E & 2 D & 2 F
\end{array}\right|
$$

Die Determinante $\Delta$ ist von $x$ und $y$ unabhängig. Ist sie von Null verschieden, so hat der Kegelschnitt keine Wendepunkte. Ist aber diese Verbindung von Konstanten identisch $=0$, so hat der Kegelschnitt an jeder Stelle die Krümmung Null und besteht dann aus einem System von zwei geraden Linien.

49) Die Wendepunkte der Kurve $y=\sin x$ zu finden.

$$
x=0, \pm \pi, \pm 2 \pi, \pm 3 \pi \text {, usw., } y=0 \text {. }
$$

\section{§ 5. Der Flăcheninhalt begrenzter Figuren.}

Um das von dem Kurvenstück $A B$, den Ordinaten $A C$ und $B D$ und dem Abschnitte $C D$ der Abscissen-Achse eingeschlossene Flächenstïck zu finden (Fig. 15), teilen wir

Fig. 15.

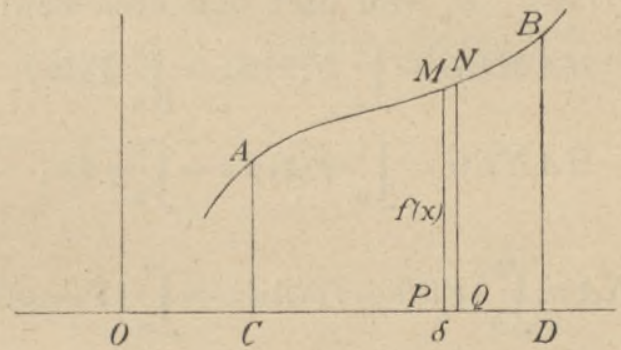

$C D=(b-a)$ in $n$ gleiche Teile, deren Grösse $\delta$ sein mag, und errichten in allen Teilpunkten Ordinaten. Dadurch wird die Fläche in $n$ Streifohen zerlegt, wie $M N Q P$, deren Inhalt bei hinlänglich kleinem $\delta$ gleich dem Produkte aus $\delta$ und der zugehörigen Ordinate gesetzt werden kann. Ist also $y=f(z)$ die Gleichung der Kurve, dann entsteht für die Fläche nach (15), S. 173, der Wert F. $A B D C=\lim \delta[f(a)+f(a+\delta)+. .+f(b-\delta)]=\int_{a}^{l} f(x) d x$. 
Um ferner das von der Kurve $A M B N A$ (Fig. 16) eingeschlossene Flächenstlick zu finden, ziehen wir die beiden

Fig. 16.

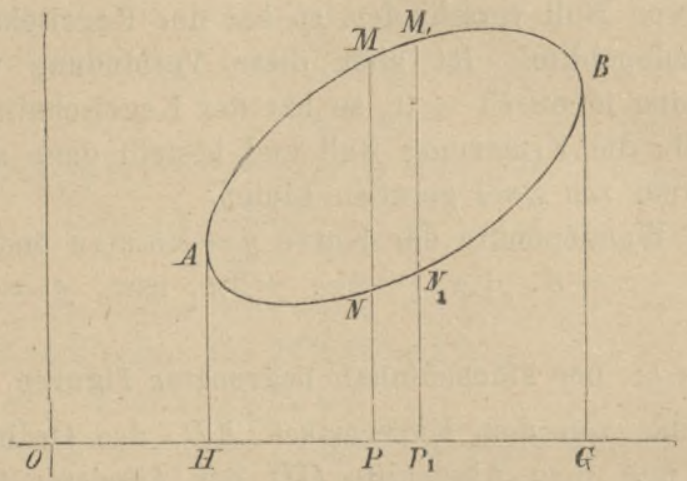

vertikalen 'Tangenten $A H$ und $B G$, zwischen denen die gesamte Kurve liegt. Ist $M P=F(x)=Y, N P=f(x)=y$, $O H=a, O G=b$, so wird nach dem eben Bewiesenen

und daher

$$
\begin{aligned}
& \text { HAMBG }=\int_{a}^{b} F(x) d x=\int_{a}^{b} Y d x, \\
& \text { HANBG }=\int_{a}^{b} f(x) d x=\int_{a}^{b} y d x,
\end{aligned}
$$

$$
\text { Fl. } A M B N A=\int_{a}^{b}[F(x)-f(x)] d x=\int_{a}^{b}(Y-y) d x \text {. }
$$

Vertauscht man die $X$-Achse mit der $Y$-Achse und setzt (Fig. 17) $O L=\alpha, O D=\beta ; B N=\Phi(y)=X, B M=\varphi(y)=x$, so erhält man

$$
\text { Fl. } A M C N A=\int_{\alpha}^{\beta}[\Phi(y)-\varphi(y)] d y=\int_{\alpha}^{\beta}(X-x) d y \text {. }
$$

Ist endlich die Gleichung der Kurve in Polarkoordinaten gegeben, so zerlege man die Fläche in elementare Streifen von der Form $M M_{1} N_{1} N$ und setze $O M=R, O N=r$, $O M_{1}=R_{1}, O N_{1}=r_{1}$, Wkl. $M O A=t$, Wkl. $M_{1} O A=t_{1}$, 
Fig, 17.

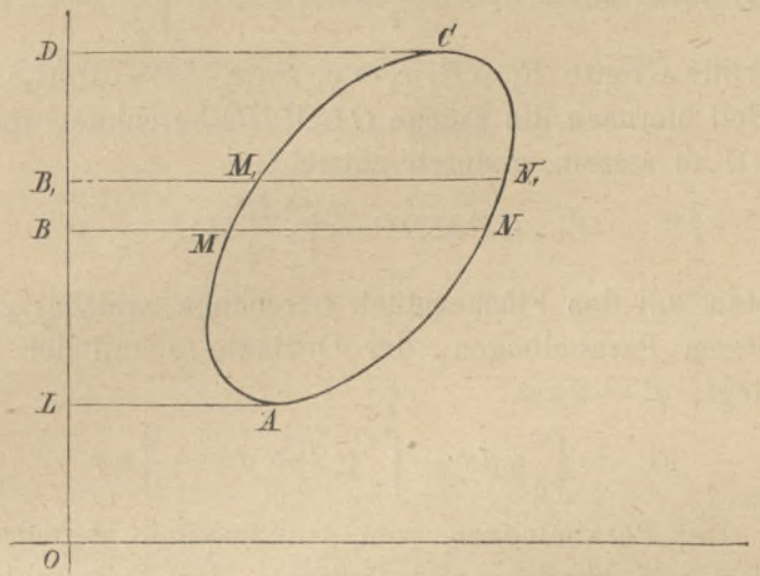

Wkl. $D O A=\gamma$, Wkl. $B O A=\delta$. Da nach der Voraussetzung der Winkel $M_{1} O M=t_{1}-t$ unmessbar klein ist, und deshalb die Bögen $M M_{1}$ und $N N_{1}$ als gerade Linien anzusehen sind, so ist:

$$
\text { Fl. } N M M_{1} N_{1}=\lim \frac{\left(R R_{1}-r r_{1}\right) \sin \left(t_{1}-t\right)}{2} \text {. }
$$

Fig. 18.

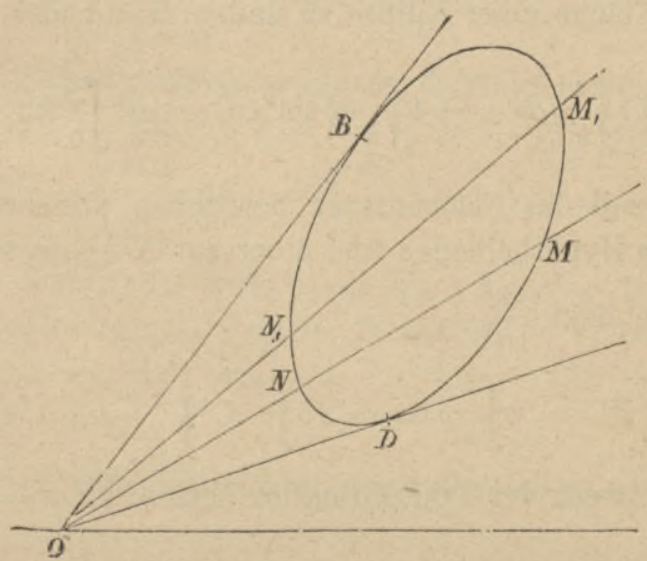


Fl. $B N D M B=\lim \frac{\Sigma\left(R R_{1}-r r_{1}\right) \sin \left(t_{1}-t\right)}{2}=\int_{\gamma}^{\delta} \frac{R^{2}-r^{2}}{2} d t$,

weil für die Grenze $R_{1}=R, r_{1}=r, \sin \left(t_{1}-t\right)=\operatorname{arc}\left(t_{1}-t\right)=d t$ wird. Soll hiernach die Fläche $O B M D O$ berechnet werden, so ist $r=0 \mathrm{zu}$ setzen, wodurch entsteht:

$$
\text { Fl. } O B M D O=\int_{\gamma}^{\delta} \frac{R^{2}}{2} d t \text {. }
$$

50) Man soll das Flächenstïck berechnen, welches zwischen einem Parabelbogen, der Ordinate $y$ und der $X$-Achse liegt; $y^{2}=2 p x$.

$$
\text { Fl. }=\int_{0}^{x} y d x=\int_{0}^{x} \sqrt{2 p x} d x=\frac{2}{3} x y .
$$

Der Parabelbogen, vom Anfangspunkt gerechnet, teilt das aus Abscisse und Ordinate des Endpunktes konstruierte Rechteck so, dass zwei Dritteile desselben die Parabelfläche bilden.

51) Die Fläche eines Kreises zu finden; $x=r \cos t, y=r \sin t$. $d x=-r \sin t d t ;$ für $x=0$ ist $t=\frac{\pi}{2}$, für $x=r$ ist $t=0$.

Fl. $=4 \int_{0}^{r} y d x=-4 \int_{\frac{\pi}{2}}^{0} r^{2} \sin ^{2} t d t=4 r^{2} \int_{0}^{\frac{\pi}{2}} \sin ^{2} t d t=r^{2} \pi$.

52) Die Fläche einer Ellipse zu finden; $x=a \cos t, y=b \sin t$. Fl. $=4 \int_{0}^{a} y d x=-4 \int_{\frac{\pi}{2}}^{0} a b \sin ^{2} t d t=4 a b \int_{0}^{\frac{\pi}{2}} \sin ^{2} t d t=a b \pi$

53) Man soll das Flächenstück berechnen, welches zwischen einem Hyperbelbogen und einer zur $X$-Achse senkrechten Sehne liegt; $\frac{x^{2}}{a^{2}}-\frac{y^{2}}{b^{2}}=1$.

$$
\text { Fl. }=2 \int_{a}^{x} y d x=2 b \int_{a}^{x} d x \sqrt{\frac{x^{2}}{a^{2}}-1} .
$$

Zum Zweck der Transformation setzen wir $x=a \frac{e^{t}+e^{-t}}{2}$, 
$y=b \frac{e^{t}-e^{-t}}{2} ;$ dies geht an, weil dadurch die Kurvengleichung befriedigt wird. Da $x=a$ für $t=0$ wird, und da $d x=a \frac{e^{t}-e^{-t}}{2} d t$ ist, so ist:

$\mathrm{Fl} .=\frac{a b}{2} \int_{0}^{t}\left(e^{t}-e^{-t}\right)^{2} d t=\frac{a b}{2}\left(\frac{e^{2 t}-e^{-2 t}}{2}-2 t\right)=x y-a b t$. Ferner ist $\frac{x}{a}+\frac{y}{b}=e^{t}$, oder $t=\lg \left(\frac{x}{a}+\frac{y}{b}\right)$. Fl. $=x y-a b \lg \left(\frac{x}{a}+\frac{y}{b}\right)$.

54) Man zeichne eine Hyperbel nebst ihren beiden Asymptoten. Kurvengleichung: $x y=\frac{a^{2}+b^{2}}{4}=m^{2}$. Es sei $A$ der Anfangspunkt des Systems, $S$ der Scheitel, $S B$ die Ordinate und $A B=p$ die Abscisse des Scheitels; $M D$ die Ordinate und $A D=x$ die Abscisse des beliebigen Punktes $M, \alpha$ der Asymptotenwinkel. Die Fläche $S B D M$ soll berechnet werden.

Weil das System schiefwinkelig ist, so ist das Differential der Fläche $=y \sin \alpha d x$, und:

$$
\text { Fl. }=\sin \alpha \int_{p}^{x} \frac{m^{2}}{x} d x=m^{2}(\lg x-\lg p) \sin \alpha .
$$

55) Die Fläche zwischen einem Cykloidenzweig und der $X$-Achse zu finden; $x=a(t-\sin t), y=a(1-\cos t)$.

$$
\text { Fl. }=\int_{0}^{2 a \pi} y d x=a^{2} \int_{0}^{2 \pi}(1-\cos t)^{2} d t=3 a^{2} \pi .
$$

56) Eine Kurve hat von $t=0$ bis $t=\pi$ die Gleichungen: $x=a(1-\cos t), y=a t$; man soll die Fläche zwischen der Kurve, der Ordinate für $t=\pi$ und der $X$-Achse finden.

$$
\text { Fl. }=a^{2} \int_{0}^{\pi} t \sin t d t=a^{2} \pi \text {. }
$$


57) Die Fläche zu berechnen, welche von einem Epicykloiden. zweige und den nach seinen Endpunkten gezogenen Halbmessern des Grundkreises eingesehlossen wird;

$$
\begin{aligned}
& x=(a+r) \cos t-a \cos \left(\frac{a+r}{a}\right) t, \\
& y=(a+r) \sin t-a \sin \left(\frac{a+r}{a}\right) t .
\end{aligned}
$$

Denken wir uns (Fig. 14, S. 182) die angefangene Kurve $E M$ so weit gezeichnet, bis sie in einem Punkte $Q$ den Grundkreis wieder trifft, so soll die Fläche $A E M Q A$ berechnet werden. Man ziehe Leitstrahl $A M=R$ und setze Wkl. $M A E=\varphi$, so ist:

$$
\text { Fl. } A E M A=\frac{1}{2} \int_{0}^{\varphi} R^{2} d \varphi \text {. }
$$

Da aber $R^{2}=x^{2}+y^{2}, \operatorname{tg} \varphi=\frac{y}{x}, \cos ^{2} \varphi=\frac{x^{2}}{x^{2}+y^{2}}$, wenn $x, y$ die Koordinaten von $M$ sind, so ist:

$\frac{d \operatorname{tg} \varphi}{d t}=\frac{d \frac{y}{x}}{d t}, \quad$ oder $\frac{1}{\cos ^{2} \varphi} \frac{d \varphi}{d t}=\frac{x \frac{d y}{d t}-y \frac{d x}{d t}}{x^{2}}$,

$\frac{d \varphi}{d t}=\frac{x \frac{d y}{d t}-y \frac{d x}{d t}}{R^{2}}=\frac{(a+r)(2 a+r)\left[1-\cos \frac{r}{a} t\right]}{R^{2}} ;$

Fl. $=\frac{1}{2} \int_{0}^{t} R^{2} \frac{d \varphi}{d t} d t=\frac{(a+r)(2 a+r)}{2} \int_{0}^{t}\left[1-\cos \frac{r}{a} t\right] d t$.

Ftur die Fläche $A E M Q A$ hat der Erzeugungskreis eine ganze Umdrehung vollendet, so dass $r t=2 a \pi$, oder $t=\frac{2 a \pi}{r}$ sein muss.

Fl. $\begin{aligned} A E M Q A=\frac{(a+r)(2 a+r)}{2} \int_{0}^{\frac{2 a \pi}{r}}\left[1-\cos \frac{r}{a} t\right] d t \\ =\frac{(a+r)(2 a+r) a \pi}{r} .\end{aligned}$

Wird davon der Kreisaussehnitt $A E Q A=a r \pi$ abgezogen, so bleibt die Fläohe $E M Q B E$ zurlick. 
58) Die Kardioidenfläche erhält man aus der vorhergehenden Formel, indem man $r=a$ setzt. Fl. $=6 a^{2} \pi$.

59) Die Fläche der Lemniskate: $R^{2}=a^{2} \cos 2 t$ zu finden.

$$
\text { Fl. }=2 a^{2} \int_{0}^{\frac{\pi}{4}} \cos 2 t d t=a^{2} \text {. }
$$

60) Die Fläche der archimedischen Spirale: $R=a t$ soll für den ersten Umlauf des Leitstrahls gefunden werden.

$$
\text { Fl. }=\frac{a^{2}}{2} \int_{0}^{2 \pi} t^{2} d t=\frac{4 a^{2} \pi^{3}}{3} .
$$

\section{§6. Rektifikation ebener Kurven.}

Sind $M$ und $M_{1}$ zwei beliebige Kurvenpunkte; $x, y$ und $x_{1}, y_{1}$ ihre Koordinaten, so ist die Sehne:

$$
M M_{1}=s=\sqrt{\left(y-y_{1}\right)^{2}+\left(x-x_{1}\right)^{2}}=\left(x-x_{1}\right) \sqrt{1+\left(\frac{y-y_{1}}{x-x_{1}}\right)^{2}} .
$$

Beim Grenziibergang erhält man als Differential des Bogens:

$$
d s=d x \sqrt{1+\left(\frac{d y}{d x}\right)^{2}} .
$$

Sind $\alpha$ und $a$ die Abscissen der Grenzpunkte $A$ und $B$, so ist:

$$
S=\operatorname{arc} A B=\int_{\alpha}^{a} d x \sqrt{1+\left(\frac{d y}{d x}\right)^{2}} .
$$

Soll aber are $A B$ aus den Ordinaten der Endpunkte berechnet werden, so setze man:

$$
s=\sqrt{\left(x-x_{1}\right)^{2}+\left(y-y_{1}\right)^{2}}=\left(y-y_{1}\right) \sqrt{1+\left(\frac{x-x_{1}}{y-y_{1}}\right)^{2}},
$$

woraus beim Grenzübergang entsteht: $d s=d y \sqrt{1+\left(\frac{d x}{d y}\right)^{2}}$, und, wenn $\beta$ und $b$ die Ordinaten der Endpunkte $A, B$ sind:

$$
S=\operatorname{arc} A B=\int_{\beta}^{b} d y \sqrt{1+\left(\frac{d x}{d y}\right)^{2}} .
$$

Für die Gleichungsform: $f(x, y)=0$ wird aus (49):

$$
S=\int_{\alpha}^{a} d x \sqrt{\left(\frac{\partial f}{\partial x}\right)^{2}+\left(\frac{\partial f}{\partial y}\right)^{2}}: \frac{\partial f}{\partial y}
$$

Dölp, Aufgaben. 
und daraus wieder, weil $\frac{\partial f}{\partial x} d x=-\frac{\partial f}{\partial y} d y$ ist,

$$
S=-\int_{\beta}^{b} d y \sqrt{\left(\frac{\partial f}{\partial x}\right)^{2}+\left(\frac{\partial f}{\partial y}\right)^{2}}: \frac{\partial f}{\partial x} .
$$

Ist endlich die Kurve durch die beiden Gleichungen: $x=\varphi(t), y=\phi(t)$ gegeben, und ist weiter $\alpha=\varphi(\gamma), a=\varphi(c)$, so' geht (49) über in:

$$
S=\int_{\gamma}^{c} d t \sqrt{\left(\frac{d y}{d t}\right)^{2}+\left(\frac{d x}{d t}\right)^{2}} .
$$

61) Die Peripherie eines Kreises zu berechnen; $x=r \cos t$, $y=r \sin t$.

$$
S=\int_{0}^{2 \pi} d t \sqrt{\left(\sin ^{2} t+\cos ^{2} t\right) r^{2}}=2 r \pi
$$

62) Die Länge eines Parabelbogens vom Scheitel bis zum Punkt $x, y$ zu finden; $y^{2}=2 p x$.

$S=\int_{0}^{y} d y \sqrt{1+\frac{y^{2}}{p^{2}}}=\frac{y}{2 p} \sqrt{y^{2}+p^{2}}+\frac{p}{2} \lg \left[\frac{y+\sqrt{y^{2}+p^{2}}}{p}\right]$.

63) Den Bogen einer Neilschen Parabel zu finden, vom Anfangspunkt bis zum Punkt $x, y ; y^{2}=a x^{3}$.

$$
S=\int_{0}^{x} d x \sqrt{1+\frac{9 a x}{4}}=\frac{8}{27 a}\left[\left(1+\frac{9 a x}{4}\right)^{\frac{3}{2}}-1\right] .
$$

64) Der Bogen einer Kettenlinie vom tiefsten Punkte bis zum Punkt $x, y$ soll berechnet werden; $y=\frac{m}{2}\left(e^{\frac{x}{m}}+e^{-\frac{x}{m}}\right)$. $S=\frac{1}{2} \int_{0}^{x} d x \sqrt{4+\left(e^{\frac{x}{m}}-e^{-\frac{x}{m}}\right)^{2}}=\frac{m}{2}\left(e^{\frac{x}{m}}-e^{-\frac{x}{m}}\right)=\sqrt{y^{2}-m^{2}}$. Der Bogen einer Kettenlinie, genommen vom tiefsten Punkte bis zum Punkt $x, y$, ist gleich der zweiten Kathete eines rechtwinkeligen Dreiecks, dessen eine Kathete $=m$ die Ordinate des tiefsten Punktes, und dessen Hypotenuse die Ordinate des Endpunktes ist.

65) Es soll die Bogenlänge der Cykloide gefunden werden; $x=a(t-\sin t), y=a(1-\cos t)$. 


$$
S=a \int_{0}^{2 \pi} d t \sqrt{2(1-\cos t)}=2 a \int_{0}^{2 \pi} \sin \frac{t}{2} d t=8 a .
$$

66) Die Bogenlänge einer Epicykloide zu berechnen.

$$
\begin{array}{r}
\left(\frac{d x}{d t}\right)^{2}+\left(\frac{d y}{d t}\right)^{2}=4(a+r)^{2} \sin ^{2} \frac{r}{2 a} t . \\
S=2(a+r) \int_{0}^{\frac{2 a \pi}{r}} \sin \frac{r}{2 a} t d t=\frac{8 a(a+r)}{r} .
\end{array}
$$

Setzt man $a=r$, so erhält man $16 a$ als Länge der Kardioide.

67) Die Länge des Bogens der archimedischen Spirale zu finden; $r=a t$.

$$
S=a \int_{0}^{t} \sqrt{1+t^{2}} d t=\frac{a}{2}\left[t \sqrt{1+t^{2}}+\lg \left(t+\sqrt{1+t^{2}}\right)\right] .
$$

68) Den Umfang einer Ellipse zu berechnen; $x=a \cos t$, $y=b \sin t$.

Ein Quadrant ist gleich:

$$
\begin{aligned}
S & =\int_{0}^{\frac{\pi}{2}} d t \sqrt{a^{2} \sin ^{2} t+b^{2} \cos ^{2} t}=a \int_{0}^{\frac{\pi}{2}} d t \sqrt{1-\frac{a^{2}-b^{2}}{a^{2}} \cos ^{2} t} \\
& =a \int_{0}^{\frac{\pi}{2}} d t \sqrt{1-\varepsilon^{2} \cos ^{2} t}, \text { wenn } \frac{a^{2}-b^{2}}{a^{2}}=\varepsilon^{2} \text { ist. } \\
S & =a \int_{0}^{\frac{\pi}{2}}\left[1-\frac{1}{2} \varepsilon^{2} \cos ^{2} t-\frac{1}{8} \varepsilon^{4} \cos ^{4} t-\frac{1}{16} \varepsilon^{6} \cos ^{6} t-\ldots\right] d t ; \\
S & =\frac{a \pi}{2}\left[1-\left(\frac{1}{2}\right)^{2} \varepsilon^{2}-\left(\frac{1}{2.4}\right)^{2} 3 \varepsilon^{4}-\left(\frac{1.3}{2.4 .6}\right)^{2} 5 \varepsilon^{6}-\ldots\right] .
\end{aligned}
$$

\section{§ 7. Die Oberflãche von Rotationskörpern.}

Wird eine Kurve um ihre X-Achse gedreht, so erzeugt sie eine krumme Oberfläche. Ist die Ordinate eines sich drehenden Kurvenelements $=y$, so ist das Differential der Oberfläche gleich der Oberfläche eines kleinen Cylindermantels, dessen Umfang $=2 y \pi$, und dessen Seite $=d s$, d. h. gleich 
dem Differential des Bogens ist. Hiernach ist das Differential der Oberfläche $d O=2 y \pi d s$, und wenn $a$ und $b$ die Anfangsund die Endabscisse des Oberflächenstückes sind,

$$
O=2 \pi \int_{a}^{b} y \sqrt{1+\left(\frac{d y}{d x}\right)^{2}} d x
$$

69) Die Oberfläche einer Kugelhaube zu finden, wenn

$$
y=\sqrt{2 r x-x^{2}}
$$

die Scheitelgleichung des Erzeugungskreises, und $h$ die Höhe der Haube ist.

$$
O=2 \pi \int_{0}^{h} d x \sqrt{2 r x-x^{2}} \sqrt{1+\frac{(r-x)^{2}}{2 r x-x^{2}}}=2 r \pi h .
$$

70) Die Mantelfläche eines Kegels zu berechnen.

Ist $y=k x$ die Gleichung der Geraden, die durch Umdrehung den Kegelmantel erzeugt, und $h$ die Höhe des Kegels, so ist:

$$
O=2 k \pi \sqrt{1+k^{2}} \int_{0}^{h} x d x=k \pi h^{2} \sqrt{1+k^{2}} .
$$

Da $k=\operatorname{tg} \alpha$, wenn $\alpha$ der Richtungswinkel der erzeugenden Geraden, so ist $k h=r=$ Basishalbmesser, und $h \sqrt{1+k^{2}}=s=$ Länge der Erzeugungslinie. Durch Substitution dieser Werte erhält man: $O=r \pi s$.

71) Es soll die Oberfläche des Körpers gefunden werden, der entsteht, wenn sich eine Cykloide um ihre $X$-Achse dreht. $x=a(t-\sin t), y=a(1-\cos t)$.

$$
\begin{aligned}
O=2 a^{2} \pi \int_{0}^{2 \pi}(1-\cos t) & \sqrt{2(1-\cos t)} d t \\
= & 8 a^{2} \pi \int_{0}^{2 \pi} \sin ^{3} \frac{t}{2} d t=\frac{64 a^{2} \pi}{3} .
\end{aligned}
$$

72) Die Oberfläche eines Rotations-Paraboloides zu berechnen. Parabelgleiehung: $y^{2}=2 p x$.

$$
O=2 \pi \sqrt{p} \int_{0}^{x} d x \sqrt{p+2 x}=\frac{2 \pi \sqrt{p}}{3}\left[(p+2 x)^{\frac{3}{2}}-p^{\frac{3}{2}}\right] .
$$

73) Man soll die Oberfläche eines Ellipsoides berechnen, das entsteht, wenn sich eine Ellipse um eine ihrer Achsen dreht. 
Erstens: Drehung um die grosse Achse.

Ist $y^{2}=\frac{b^{2}}{a^{2}}\left(a^{2}-x^{2}\right)$ die Gleichung der Kurve, so ist $\frac{1}{2} O=\frac{2 b \pi}{a^{2}} \int_{0}^{a} d x \sqrt{a^{4}-\left(a^{2}-b^{2}\right) x^{2}}$.

Wir setzen $a^{2}-b^{2}=e^{2}$ und erhalten:

$$
\begin{aligned}
& \frac{1}{2} O=\frac{2 b \pi}{a^{2}} \int_{0}^{a} d x \sqrt{a^{4}-e^{2} x^{2}} \\
& \frac{1}{2} O=\frac{b \pi}{a^{2}}\left[x \sqrt{a^{4}-e^{2} x^{2}}+\frac{a^{4}}{e} \arcsin \frac{e x}{a^{2}}\right]_{0}^{a} \\
& \frac{1}{2} O=b^{2} \pi+\frac{a^{2} b \pi}{\sqrt{a^{2}-b^{2}}} \arcsin \frac{\sqrt{a^{2}-b^{2}}}{a} .
\end{aligned}
$$

Zweitens: Drehung um die kleine Achse.

$$
\begin{aligned}
& \frac{1}{2} O=\frac{2 a \pi}{b^{2}} \int_{0}^{b} d y \sqrt{b^{4}+e^{2} y^{2}} \\
& \frac{1}{2} O=\frac{a \pi}{b^{2}}\left[y \sqrt{b^{4}+e^{2} y^{2}}+\frac{b^{4}}{e} \lg \left(e^{2} y+e \sqrt{\left.b^{4}+e^{2} y^{2}\right)}\right]_{0}^{b}\right. \\
& \frac{1}{2} O=a^{2} \pi+\frac{a b^{2} \pi}{\sqrt{a^{2}-b^{2}}} \lg \frac{a+\sqrt{a^{2}-b^{2}}}{b} .
\end{aligned}
$$

Was wird aus beiden Resultaten für $b=a$ ?

\section{§ 8. Der Kubikinhalt von Rotationskörpern.}

Wird ein Körper dadurch erzeugt, dass sich die zwischen einer Kurve und der $X$-Achse gelegene Fläche um diese $X$-Achse dreht, so ist das Differential des Volumens $d V=y^{2} \pi d x$, und das Volumen zwischen den Abseissen $\alpha$ und $a$ selbst

$$
V=\pi \int_{\alpha}^{a} y^{2} d x .
$$

74) Den Kubikinhalt einer Kugel zu berechnen.

$$
V=2 \pi \int_{0}^{r}\left(r^{2}-x^{2}\right) d x=\frac{4}{3} r^{3} \pi .
$$


75) Den Kubikinhalt eines Rotationsellipsoides zu finden, das entsteht, wenn sich eine Ellipse um ihre grosse Achse dreht.

$$
V=\frac{2 \pi b^{2}}{a^{2}} \int_{0}^{a}\left(a^{2}-x^{2}\right) d x=\frac{4 a b^{2} \pi}{3} .
$$

76) Der Inhalt eines parabolischen Zonenkörpers soll berechnet werden.

Sind $x_{1}$ und $x_{2}$ die Abscissen der Endpunkte des Parabelbogens, welcher die erzeugende Fläche begrenzt, so ist:

$$
V=2 p \pi \int_{x_{1}}^{x_{2}} x d x=2 p \pi\left(\frac{x_{1}+x_{2}}{2}\right)\left(x_{2}-x_{1}\right),
$$

77) Man soll den Inhalt des Körpers finden, der entsteht, wenn sich die Cykloidenfläche um die $X$-Achse (Gerade, auf welcher der erzeugende Kreis rollt) dreht.

$$
V=a^{3} \pi \int_{0}^{2 \pi}(1-\cos t)^{3} d t=8 a^{3} \pi \int_{0}^{2 \pi} \sin ^{6} \frac{t}{2} d t=5 a^{3} \pi^{2} .
$$

78) Ein Kreisabschnitt, dessen Sehne $=2 s$, und dessen Halbmesser $=r$ ist, dreht sich um einen zur Sehne parallelen Durehmesser. Wie gross ist der Kubikinhalt des entstandenen Körpers?

$$
\begin{aligned}
& \frac{1}{2} \boldsymbol{V}=\pi \int_{0}^{s}\left(r^{2}-x^{2}\right) d x-s \pi\left(r^{2}-s^{2}\right) ; \\
& V=\frac{4}{3} s^{3} \pi .
\end{aligned}
$$




\section{INHALT.}

\section{Differentialrechnung.}

Funktionen einer unabhängigen Variabelen.

§ 1 Zusammenstellung der Differentialquotienten der einfachen Funktionen .................. 1

§ 2. Erklärung der Funktion einer unabhängigen Variabelen. Erklärung und Entwickelung des Differentialquotienten . 2

$\S$ 3. Aufgaben zur Differentiation algebraischer Funktionen .

§ 4. Die Differentialquotienten der trigonometrischen und cyklometrischen Funktionen . . . . . . . . . . . . 22

§ 5. Exponential- und logarithmische Funktionen . . . . . . 29

§ 6. Unentwickelte Funktionen . . . . . . . . . . . . . . 39

§ 7. Funktionen von der Form: $x=\varphi(t), y=\psi(t) \ldots 44$

$\S$ 8. Differentialquotienten höherer Ordnung . . . . . . . . 47

§ 9. Anwendung der Differentialrechnung zur Ermittelung der Werte unbestimmter Formen .......... 56

$\S$ 10. Maxima und Minima der Funktionen . . . . . . . . . 64

§ 11. Die Reihen von Taylor und Maclaurin . . . . . . . . . 91

Funktionen von zwei unabhängigen Variabelen.

§ 12. Entwickelung der Differentialquotienten . . . . . . . 100

$\S 13$. Maxima und Minima von Funktionen mit zwei unabhängigen Variabelen . . . . . . . . . . . . . 102

§ 14. Homogene Funktionen . . . . . . . . . . . . . . . 105

$\S 15$. Die Reihen von Taylor und Maclaurin für Funktionen mit zwei unabhängigen Variabelen ......... 107

\section{Integralrechnung.}

Unbestimmte Integrale.

§ 1. Die einfachen Integralformen ... . . . . . . . . . . 109

$\S$ 2. Integration rationaler algebraischer Brüche . . . . . . 116 
§ 3. Reduktionsformeìn . . . . . . . . . . . . . . . . . . . 139

§ 4. Irrationale algebraische Funktionen . . . . . . . . . . . 141

§ 5. Exponential- und logarithmische Funktionen . . . . . . . 157

§ 6. Trigonometrische und cyklometrische Funktionen . . . . 160

Bestimmte Integrale . . . . . . 170

\section{Anwendung der Differential- und Integralrechnung auf Geometrie.}

§ 1. Tangente und Normale ebener Kurven . . . . . . . . . 178

§ 2. Doppelpunkte, Rückkehrpunkte (Spitzen), konjugierte (isolierte) Punkte . . . . . . . . . . . . . . . . 184

§ 3. Krümmungskreis und Evolute . . . . . . . . . . . . . . 189

§ 4. Die Wende- oder Inflexionspunkte . . . . . . . . . 198

§ 5. Der Flächeninhalt begrenzter Figuren . . . . . . . . . . 203

§ 6. Rektifikation ebener Kurven . . . . . . . . . . . . . . 209

§ 7. Die Oberfläche von Rotationskörpern . . . . . . . . 211

§ 8. Der Kubikinhalt von Rotationskörpern . . . . . . . 213 



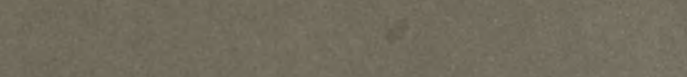

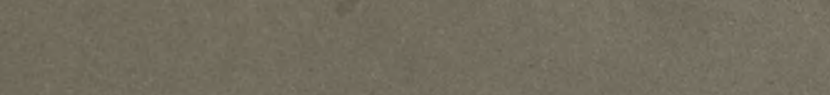

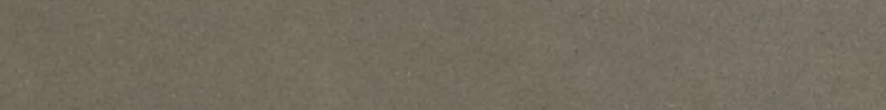

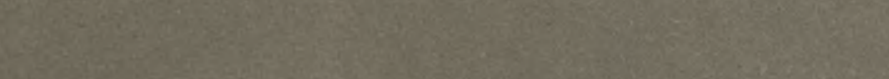

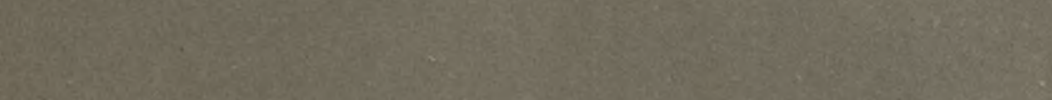

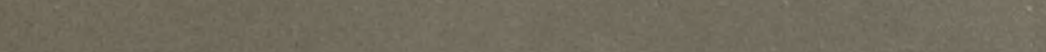
H.

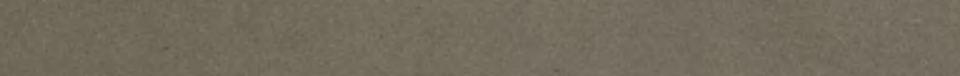

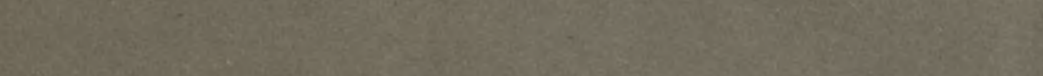

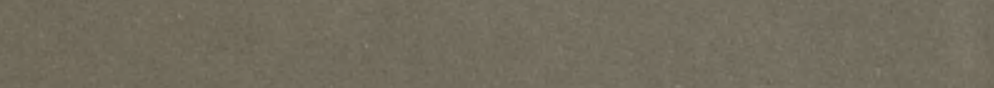

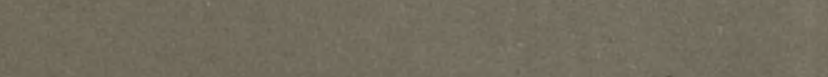
(1) If 3 -

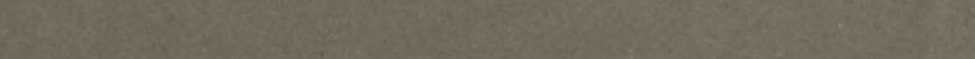

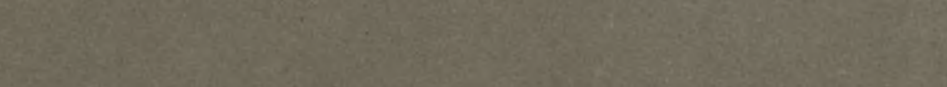

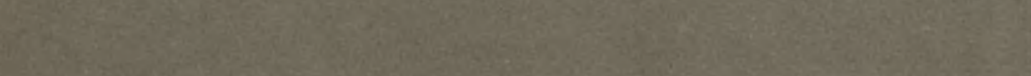

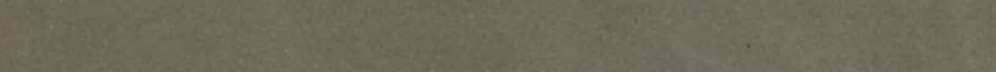

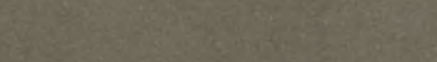
xat?

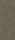
1.5. (3)

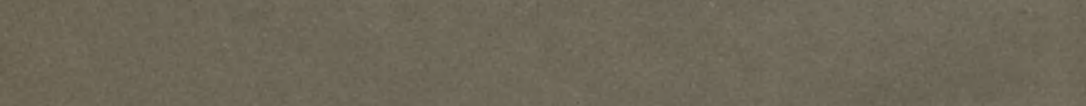

U. cosing

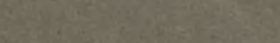

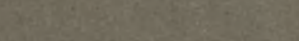
and H.

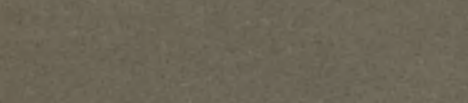
Lex tof

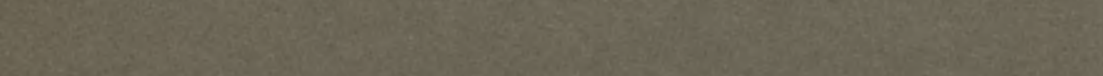
W. 

Biblioteka Politechniki Krakowskiej

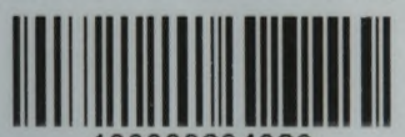

100000294389 